\title{
WiRELESS MAGNETOELASTIC MONITORING OF BILIARY STENTS
}

\author{
by \\ Scott Ryan Green \\ A dissertation submitted in partial fulfillment \\ of the requirements for the degree of \\ Doctor of Philosophy \\ (Mechanical Engineering) \\ in The University of Michigan \\ 2009
}

Doctoral Committee:

Professor Yogesh B. Gianchandani, Chair

Professor Grace H. Elta

Professor Kensall D. Wise

Associate Professor Katsuo Kurabayashi

Assistant Professor Shorya Awtar 
In Memory of

George H. Shepherd and Frank O. Green

- ii - 


\section{ACKNOWLEDGMENTS}

The work described by this dissertation was funded in part by the University of Michigan, as well as by the National Science Foundation (NSF) Wireless Integrated Microsystems (WIMS) Engineering Research Center (ERC). I was also supported for the first three years of my doctoral research by a Graduate Research Fellowship through the NSF. Of course, I would not have been able to obtain that fellowship and attend the University of Michigan without the advice and personal recommendations from Dr. Phil Cornwell, Dr. Richard Stamper, Dr. Charles Farrar, Dr. Art Western, and Doug Staunton, P.E. For their assistance and support, I am grateful.

Samples for this work were provided by Metglas, Inc.; Dow Corning; and Hoosier Magnetics. Fabrication and experimental assistance was provided by Ms. Seow Yuen Yee, Ms. Farah Shariff, and Dr. Hanseup Kim. Vibrating sample magnetometer measurements were provided by Princeton Measurements Corporation.

I would like to thank my committee members for their guidance in the completion of this research and dissertation. The conversations with Dr. Grace Elta were particularly helpful in understanding the problem and the standard usage of biliary stents. Although not a part of my committee, Dr. Richard Kwon was also instrumental in gaining an understanding of the problem and in conducting the in situ experiment described herein. Professor Yogesh Gianchandani, as chair of my committee and as the advisor of my doctoral research, provided me with intellectual freedom within a focused, well-defined and important project - one which may yield truly impactful, practical results outside of 
academia with continued development. I look forward to future collaborations with each of you.

I have had the pleasure of working with and developing friendships with many students while at the University of Michigan, particularly within the Gianchandani research group. Many good times - and of course, some trying times as deadlines approached - were had with Amar, Christine, Allan, Angelo, Naveen, Tao, Bhaskar, Jong, Karthik, Scott W., and Weibin. I especially want to express my gratitude to Mark Richardson for not only being my mentor as I began my studies - allowing me to hit the ground running - but also for being an instrumental part of grasping the fundamentals of this research. It was our efforts in the summer of 2006 that laid the groundwork for all of this, and without Mark I am sure I would still be puzzling over how to get a usable signal out of a magnetoelastic sensor.

The support of my family has been the main reason that I have come this far. My parents, Mike and Vicki Green, have always been proponents of a strong education - not for the sake of education, but because it is an avenue for becoming a complete person and an important part of society. Despite some of my natural inclinations to the contrary, they were able to imbue me with not only a strong work ethic, a logical mind and a certain amount of discipline, but also with the ability to express myself, interact with others, and find the humor in all things. I am what I am today because of you, Mom and Dad. I also want to recognize my sister Elizabeth for her selflessness and for trying to make sure I do not forget to enjoy life a little. My brother Patrick is not only a lot of fun to be around but also inspires everybody by always reaching toward fulfillment of his potential. Grandma Green and Grandma Shepherd always let me know how proud they 
are of me, and I cherish the love and unconditional support that they provide me.

Of course, there has been one true love throughout all of this - from before I knew I could be an engineer, through our maturation into adults, and into the future - and that is the love of my beautiful Jenny. Every day she is in inspiration to me with her energy and enthusiasm. She understands what drives me and she somehow even appreciates my humor. She keeps me connected to my family and to my friends even though I often find myself buried in work. Although the future is never completely clear, I know that we will enjoy wherever life may take us together. 


\section{TABLE OF CONTENTS}

DEDICATION

ACKNOWLEDGMENTS

LIST OF FIGURES $\quad$ ix

LIST OF TABLES $\quad$ XV

LIST OF APPENDICES X xvi

LIST OF SYMBOLS X Xvii

Chapter

1. Introduction 1

1.1 Stent Applications and Associated Complications 2

1.2 Potential Sensor Systems for Monitoring Stents 14

$\begin{array}{lll}1.3 & \text { Focus of this Work } & 21\end{array}$

2. Theory, Modeling, General Design Considerations, $\begin{array}{ll}\text { and Component Specifications } & 27\end{array}$

2.1 Biliary Stent Design 28

2.2 Sensor Design 33

2.3 DC Magnetic Bias Design $\quad 69$

2.4 Interrogation Module Design 72

$\begin{array}{ll}\text { 2.5 Summary and Performance Goals } & 87\end{array}$ 


\section{Second-Generation System - Patterned Sensor} and Conformal Magnetic Layer

4.1 Design

4.2 Fabrication

4.3 Experimental Methods

4.4 Results

4.5 Discussion

5. Advanced Sensor Functionality

5.2 Fabrication

5.3 Experimental Methods

5.4 Experimental Results

5.5 Discussion

6.1 Design and Fabrication

6.2 Porcine Carcass Experiment

6.3 Harvested Bile Characterization

6.4 Discussion 
7.1 Component Performance Summary and Potential Design Improvements

7.2 Long Term Future Work

7.3 Major Contributions to the Field 


\section{LIST OF FIGURES}

Figure

1-1: Pathology of biliary restenosis subsequent to stent placement.

1-2: LEFT: Cross sections of Common Bile Duct (CBD) after 4 months of indwelling of polymeric self-expanding stent.

1-3: LEFT: Histology of hyperplastic response near a bare metal stent.

1-4: Struvite-encrusted pigtail of a ureteral stent.

1-5: As the diameter of the vessel or duct increases, flow velocity becomes insensitive to stenosis over a larger range (from [DeH04]).

1-6: Current diagnostic techniques trade invasiveness and expense for a direct measurement of restenosis.

2-1: Conceptual diagram of two generations of in-vivo magnetoelastic sensing of sludge accumulation for biliary stents.

2-2: Partially deployed, braided, self-expanding commercial biliary stent (Wallstent $^{\mathrm{TM}}$, Boston Scientific).

2-3: Magnetostriction vs. applied field for various amorphous metals [Cla89, Kim99, Zha06].

2-4: A) Calculated tip displacement amplitude versus frequency for a $2 \mathrm{~mm}$

x $37.5 \mathrm{~mm} 2826 \mathrm{MB}$ sensor exposed to the listed environments, using equation (6).

2-5: TOP: Calculated induced voltage amplitude versus frequency for a $2 \mathrm{~mm}$

x $37.5 \mathrm{~mm}$ 2826MB sensor, using equation (11).

2-6: Calculated signal amplitude variation with sensor width (left) and length (right) for three different materials: 2826MB (top), 2605SA1 (middle) and 2605CO (bottom).

2-7: Calculated resonant frequency and signal amplitude response to paraffin mass loading for $2 \mathrm{~mm} \times 37.5 \mathrm{~mm}$ sensors of three different materials. 
2-8: Alternative amorphous metal materials provide similar resonant frequency sensitivity to paraffin mass loads but vastly different amplitude responses due to the differing mechanical impedance of each material.

2-9: Alternative amorphous metal materials again show vastly different amplitude responses to increasing viscosity due to the differing mechanical impedance of each material.

2-10: Measuring the center frequency and resonant - anti-resonant bandwidth along with the relationships presented in equations (19) and (20) allow shifts in the viscosity-density product to be estimated separately from the effects of mass loading.

2-11: FEA calculated and experimentally measured voltage amplitude (top) and phase (bottom) frequency responses for $2 \mathrm{~mm} \times 37.5 \mathrm{~mm} 2826 \mathrm{MB}$ (left) and 2605CO (right) sensors.

2-12: FEA calculated signal amplitude variation with sensor width (left) and length (right) for three different materials: 2826MB (top), 2605SA1 (middle) and 2605CO (bottom).

2-13: Effects of long indwelling in bile were simulated by bathing sensors in saline at an elevated temperature $\left(60-65^{\circ} \mathrm{C}\right)$.

2-14: Visual evidence of corrosion is present after 6 equivalent months (13 days) of accelerated aging wherever the sensor is not protected by parylene.

2-15: Experimentally measured effect of forcing longitudinal curvature on an otherwise straight $2 \mathrm{~mm} \times 37.5 \mathrm{~mm} 2826 \mathrm{MB}$ ribbon sensor.

2-16: LEFT: The red trace shows a large frequency shift of up to $2 \mathrm{kHz}$ occurs when the relative DC field orientation varies.

2-17: Coil configurations used in this work.

2-18: Normalized signal amplitude for a $37.5 \times 6 \mathrm{~mm}^{2}$ sensor at various locations to characterize the extracorporeal configuration (B) performance.

2-19: Extracorporeal configuration (C) as fabricated, with important dimensions.

2-20: Spatial characterization of extracorporeal configuration (C).

2-21: Shifts in baseline amplitude due to presence of various portions of a human body.

2-22: Shifts in baseline amplitude due to presence of various small metal objects. 
2-23: Shifts in baseline amplitude due to presence of various pieces of lab furniture.

3-1: Conceptual sketch of the first-generation system.

3-2: A) Integrated sensor attachment points before and after folding out of the plane of the stent sidewall.

3-3: Photochemical machining (PCM) process flow.

3-4: Conceptual schematic of in-stent permanent magnet configuration and resulting field lines.

3-5: Magnetostatic FEA shows a uniform field of $\sim 3$ Oe directed along the length of the sensor with this magnet configuration.

3-6: Neodymium magnet lapped from $800 \mu \mathrm{m}$ to $400 \mu \mathrm{m}$, on tip of tweezers.

3-7: A, B) Conceptual drawing and image of seam closure method 1.

3-8: Fully-assembled device, shown with a US dime.

3-9: Normalized resonant frequency response to viscosity changes.

3-10: Resonant frequency response to paraffin mass loads.

4-1: Conceptual sketch of the second-generation system.

4-2: LEFT: A portion of a wishbone-array sensor, along with important dimensions.

4-3: FEA calculated strain in a single wishbone cell during cell deformation.

4-4: FEA calculated and experimentally measured amplitude (right) and phase (left) frequency response for an unloaded planar wishbone-array sensor.

4-5: FEA calculated mode shapes for a planar wishbone-array magnetoelastic sensor, occurring at the listed (predicted) resonant frequencies.

4-6: Switchback stent design layout with important dimensions.

4-7: Curvature is achieved by placing the sensor inside a tube and annealing at $375^{\circ} \mathrm{C}$ for 30 minutes. 
4-8: The edges of the stent seam can be brought into alignment in a fixture, and subsequently encased in a layer of PDMS (the outline of which is denoted by the dashed lines).

4-9: An alternative seam closure method involves threading the integrated wire through the loops on both sides of the seam.

4-10: Fabrication process.

4-11: LEFT: Assembled sensor, stent, and magnet layer, inside a plastic tube.

4-12: Evaluation of four as-cast planar wishbone-array sensors.

4-13: Characterization of wishbone-array sensors after various heat treatments.

4-14: The wishbone-array sensor amplitude and frequency show insignificant changes after strains of up to $37 \%$.

4-15: Amplitude and resonant frequency response to viscosity for as-cast and thermally treated wishbone-array sensors.

4-16: As-cast planar and thermally treated curved wishbone-array sensors, as well as ribbon sensors $(2826 \mathrm{MB}, 2 \mathrm{~mm} \times 37.5 \mathrm{~mm})$, were loaded with either paraffin or acrylate terpolymer layers to simulate sludge accumulation.

4-17: M-H hysteresis curves for $4 \mathrm{~mm}$ diameter, $100 \mu \mathrm{m}$ thick discs of $\mathrm{SrFe}-\mathrm{PDMS}$ in different ratios.

4-18: LEFT: 3x self-expansion of fully-connected stent design.

4-19: Response of the system to viscosity changes as mass accumulates.

4-20: Response of the system to mass buildup.

5-1: Sensor design layouts and important dimensions.

5-2: FEA simulated and experimentally measured data.

5-3: FEA simulated mode shapes for the spatially sensitive design, along with predicted resonant frequencies.

5-4: Changes in sensor amplitude (left) and phase (right) response after the listed treatments applied to the hybrid ribbon design.

5-5: Sensors integrated with commercial self-expanding biliary stents $\left(8 \mathrm{~mm} \times 60 \mathrm{~mm}\right.$, Boston Scientific Wallstent $\left.{ }^{\mathrm{TM}}\right)$. 
5-6: Paraffin loading results for the spatially sensitive sensor.

5-7: Paraffin loading results for the varying feature density design.

5-8: Separation of effects of viscosity from those of paraffin mass loads for varying feature density design.

5-9: Paraffin loading results for the hybrid ribbon design.

5-10: Separation of effects of viscosity from those of paraffin mass loads for the hybrid ribbon design.

5-11: Gelatin loading results for the hybrid ribbon design.

6-1: Conceptual sketch of the magnetoelastic monitoring system used in the porcine carcass in situ experiment.

6-2: Partially deployed $8 \mathrm{~mm} \times 60 \mathrm{~mm}$ self-expanding biliary stent (Wallstent ${ }^{\mathrm{TM}}$, Boston Scientific).

6-3: Biliary stent after integration of sensor and conformal magnetic layer.

6-4: A makeshift biliary duct of approximately $6 \mathrm{~mm}$ in diameter is formed using a portion of the small intestine and a clamp.

6-5: The coils are stabilized over the animal using overhanging metal trays.

6-6: A) Measured amplitude shift from baseline for the implanted sensor at a distance of $5 \mathrm{~cm}$ from the face of the coils.

6-7: A) Measured amplitude shift from baseline for the implanted sensor at a distance of $2 \mathrm{~cm}$ from the face of the coils.

6-8: The sensor response in harvested bile is compared to the response of the sensor in fluids of known properties, showing that the viscosity of the bile can be estimated and is within the range of fluids used in previous tests.

A-1: Newton's Second Law can be applied to an infinitesimal cross section of the loaded sensor.

A-2: Examples of shape functions for two different materials.

B-1: Piezomagnetic disc layout.

B-2: LEFT: Pressure amplitude of 30-layer stack, operating at resonance in water. 
B-4: LEFT: Deformation of ultrasound device, with out of plane deflection (deformation not to scale).

B-6: Active areas are elevated out of plane using thermal annealing (1 hour at $375^{\circ} \mathrm{C}$ while a fixture provides the desired final shape).

B-7: Microphone response for different test conditions.

B-8: LEFT: Estimated deformation amplitude of prototype at resonance. 


\section{LIST OF TABLES}

Table

2-1: As-cast material properties for various Metglas $^{\mathrm{TM}}$ alloys 36

2-2: Definition of parameters in equation (4) 40

2-3: Coil configuration comparison $\quad 80$

2-4: Desired component performance $\quad 88$

4-1: Stent mechanical testing results comparison 127

5-1: Calculated mass distribution using measured characteristic frequencies 152

7-1: Component performance summary 177

A-1: Selected values of parameters A and B used in analytical model 206

A-2: Material constants fitted to experimental results for various Metglas ${ }^{\mathrm{TM}}$ alloys 216

A-3: Selected results for ribbon sensors using the analytical and FEA models 224 


\section{LIST OF APPENDICES}

\section{Appendix}

A: Derivation and Implementation of Analytical and Finite Element Models

B: Wireless Magnetoelastic Ultrasound Generation 


$$
\begin{aligned}
& \vec{\sigma}-\text { stress vector } \\
& {[C]-\text { stiffness matrix }} \\
& \vec{\varepsilon}-\text { strain vector } \\
& {[d] \text { - magnetostrictivity matrix }} \\
& d \text { - isotropic magnetostrictivity } \\
& \mu_{0}-\text { permeability of free space } \\
& \mu_{r} \text { - relative permeability } \\
& \vec{H} \text { - magnetic field strength vector } \\
& \vec{B} \text { - magnetic flux density vector } \\
& E_{a p p}-\text { apparent Young's modulus } \\
& E_{o} \text { - zero-bias Young's modulus } \\
& H_{D C} \text { - bias magnetic field } \\
& L \text { - sensor half-length } \\
& v \text { - fluid kinematic viscosity } \\
& u \text { - local sensor displacement } \\
& \omega \text { - input signal radial frequency } \\
& \varphi \text {-shape function } \\
& E_{\text {sludge }}^{*} \text { sludge complex modulus } \\
& \alpha \text {-sensor mass damping factor } \\
& \beta \text { - sensor stiffness damping factor }
\end{aligned}
$$

\section{LIST OF SYMBOLS}


$\mu$ - fluid dynamic viscosity

$t_{\text {sens }}-$ sensor thickness

$t_{\text {sludge }}$ - sludge thickness

$\rho_{\text {sens }}-$ sensor density

$\rho_{f l}-$ fluid density

$\rho_{\text {sludge }}$ - sludge density

$w$ - sensor width

$H$ - input magnetic field strength

$A$ - fitting parameter in shape function

$B$ - fitting parameter in shape function

$\lambda_{n}-\mathrm{n}^{\text {th }}$ eigenvalue

$V_{\text {sens }}-$ voltage induced in an ideal pickup coil around the sensor

$V_{\text {coil }}$ - voltage induced on a coaxially located pickup coil

$N$ - number of turns per meter length of pickup coil

$S$ - numerical value of spatial triple integral result

$r_{\text {coil }}$ - radius of pickup coil

$L_{\text {coil }}$ - half-length of pickup coil

$Q$ - quality factor

$\omega_{r}-$ resonant angular frequency

$f_{o}$ - characteristic frequency (at minimum of phase response)

$\omega_{o}-$ characteristic angular frequency (at minimum of phase response)

$\Delta \phi$ - magnitude of phase response

$\Delta f$ - half-power bandwidth of phase response 
$M_{o}-$ unloaded sensor mass

$\Delta m$ - added mass

$C$ - fitting parameter in viscosity-density product frequency response relationship

$D$ - fitting parameter in viscosity-density product frequency response relationship

$g$ - function relating bias field strength to the incremental modulus change

$v_{s}-$ sensor Poisson's ratio

$E^{\prime}-$ Young's modulus at constant magnetic field

$d^{\prime}$ - magnetostrictivity at constant magnetic field

$\mu_{r}^{\prime}-$ permeability at constant magnetic field

$A_{\text {sens }}$ - cross-sectional area of sensor

$A_{\text {sludge }}$ - cross-sectional area of sludge

$m_{f l}-$ activated fluid mass per unit length

$F_{\text {struc }}$ - damping force due to structural losses

$F_{\text {damp }}$ - damping force due to surrounding viscous media

$V_{\text {fluid }}$ - velocity of fluid

$\tau$ - shear stress

$K E_{\text {fluid }}$ - kinetic energy of fluid

$\psi_{n}-\mathrm{n}^{\text {th }}$ eigenfunction

$\left[C^{\prime}\right]$ - compliance matrix 


\section{Chapter 1}

\section{Introduction}

Stents are tubular scaffolds used to prop open various ducts and vessels within the human body that have become narrowed due to various stenotic pathologies. The stents are usually deployed in a percutaneous, catheter-based procedure; as such, the main benefit of these devices is their minimized invasiveness compared with open surgery. Prevalent stent deployment location areas are the cardiovascular space (e.g. coronary and renal arteries), the bile duct, and the ureter, among others. Depending on the intended deployment location, the stent may take on slightly different designs in terms of method of expansion (self-expanding or balloon-assisted), structural pattern (usually some variation of an open mesh), and materials utilized (metals, polymers, or some combination thereof). However, the basic function of all stents remains mechanical in nature and in general the designs are judged on how long the treated duct or vessel remains patent after deployment of the stent.

As with any medical treatment, there are complications associated with the implanted stent. The exact nature of the complications varies depending on the location of the stent, but the basic result is the same: the treated vessel or duct becomes occluded. In many cases, this occlusion occurs progressively and over a timeframe that varies greatly from patient to patient. Also, the progression of the occlusion may be difficult to detect - or 
alternatively may only be detectable after damage has occurred - with current techniques. As such, an opportunity lies in developing improved techniques for monitoring the continued patency of the treated area.

In section 1.1, various stent applications and the complications associated with them are explored in more detail while pointing out opportunities for improved monitoring techniques. Section 1.2 discusses advantages and disadvantages of various sensing methodologies that might be used in integrated stent monitoring techniques - i.e., systems in which the sensors are integrated with the stent. In section 1.3, the focus and structure of this work is discussed and past work in magnetoelastic sensors is highlighted with a view toward application in stent monitoring.

\subsection{Stent Applications and Associated Complications}

\subsubsection{Biliary Stents}

The bile duct carries bile between the liver, gallbladder, pancreas, and small intestine. Bile is used in the intestinal tract for the emulsification and absorption of fats, and flows through the biliary tree mainly due to contractions of the gallbladder but also due to some peristalsis of the smooth muscle lining the bile duct. The biliary stricture relieved by biliary stent implantation is often due to pancreatitis, cholangitis, tumors, surgical injury or duct breakdown due to gallstones. Pancreatitis and cholangitis are both inflammatory diseases, with pancreatitis affecting the pancreas and cholangitis affecting the bile duct system directly. In the absence of a tumor, the stricture is formed by collagen deposition and duct tissue fibrosis [Cla02, Lib96, Fra96]. 
To treat the strictures, both plastic (typically polyethylene) and metal (typically nickel-titanium or chrome-nickel) stents are available. Metal stents are typically selfexpanding meshes. Plastic stents are usually simple tapered tubes with laterallyprotruding flaps to prevent migration. Metal stents have the advantage of slightly longer patency compared to plastic stents, likely due to the capability of the stents to provide larger diameter dilation. However, metal stents are normally used only when the obstruction is malignant (cancerous), as the large expansive force of current designs results in a stent that is difficult to remove if the stent is no longer required (or no longer functioning). In addition, metal stents usually have a more open structure than plastic stents, so tumor ingrowths can sometimes be problems. This problem may be mitigated by covering the stent with an elastomeric (e.g. silicone or polyurethane) membrane [Lib96, Koz00, McL89, Som06, Dav92].

The most prevalent complication with biliary stents is late occlusion, or restenosis. Two main restenotic processes are prevalent in biliary stents. First, occlusion can occur in an average of 4-5 months in $30-75 \%$ of patients via formation of a bacterial matrix on and around the stent. This bacterial matrix is known as biliary "sludge" [Don 07, Sun95]. The pathology of sludge formation (Fig. 1-1) begins with the formation of a protein layer - including fibronectin and collagen - on the stent struts. Bacteria present in the bile, including Escherichia coli and Enterococcus, tend to adhere to the protein. As these bacteria congregate, they produce an extracellular biofilm and matrix. Additionally, the bacteria release enzymes that precipitate crystals of cholesterol and calcium bilirubinate (among others) out of solution; these crystals become trapped in the matrix. Collectively, the matrix, crystals, biofilm, and bacteria form the sludge. This sludge accumulates and 
eventually leads to occlusion of the duct which of course is the same situation that required the stent implantation (Fig. 1-2). Once the duct is occluded, the bile will back up into the liver and cause jaundice or worse - cholangitis, liver damage, or morbidity. Though it is noted in the literature that occlusion due to sludge formation is more prevalent on plastic stents, metal stents are not immune to the problem [Dav92, Cla02]. Current medical techniques for dealing with sludge accumulation include either replacing the stent while clearing the sludge or simply implanting a second stent inside the first to compact the sludge against the sidewall of the duct.

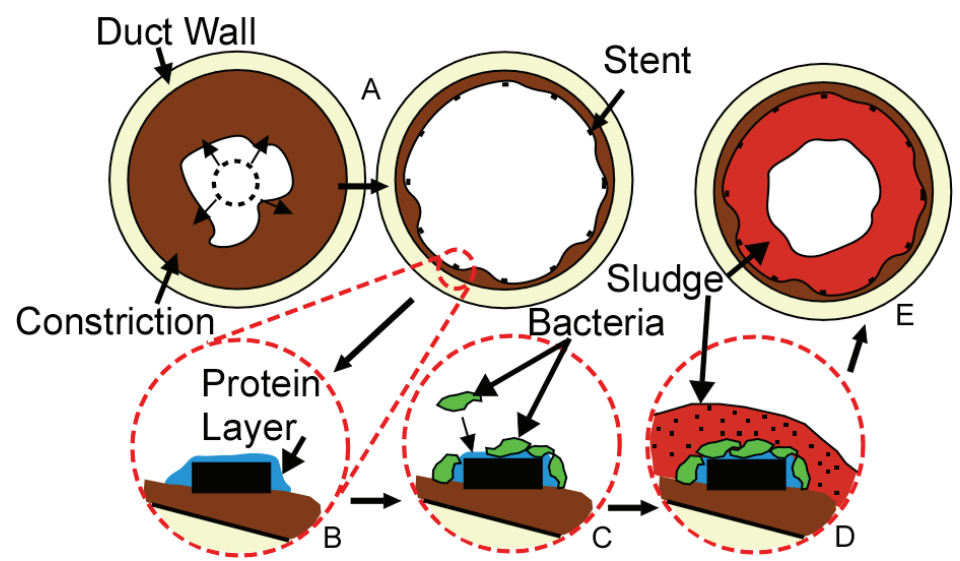

Fig. 1-1: Pathology of biliary restenosis subsequent to stent placement. A) Selfexpanding stent initially relieves duct constriction. B) A protein layer forms on the surfaces of the stent. C) The protein layer allows bacteria to adhere to the stent surfaces. D) The bacteria generate a mucopolysaccharide matrix, commonly termed "biliary sludge". E) The sludge accumulates, leading to a narrowed duct condition.

The second main occlusive process for biliary stents is submucosal hyperplasia (proliferation of the submucosal cells on the stent surfaces). The hyperplastic response is described in more detail in section 1.1.3. Submucosal hyperplasia is prevalent in metal stents and generally does not occur with plastic stents. Generally, occlusion in a metal stent is a combination of hyperplasia and sludge accumulation. 


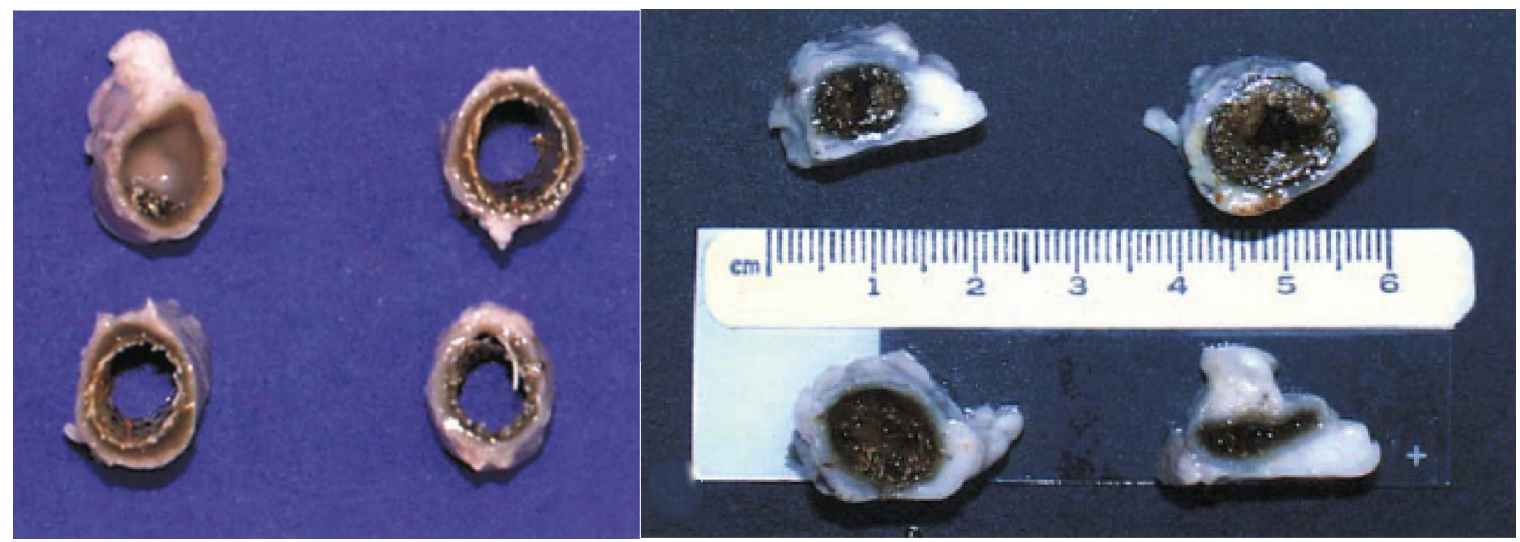

Fig. 1-2: LEFT: Cross sections of Common Bile Duct (CBD) after 4 months of indwelling of polymeric self-expanding stent. RIGHT: Cross sections of CBD after 6 months of stent indwelling. Note the presence of biliary sludge (from [Gin03]).

Various methods for the prevention of biliary stent clogging have been investigated [Lib96, Sun95, Sei07, Don07, DeL00]. These methods include using stent materials with different levels of hydrophilicity or even materials impregnated with antimicrobial agents. Alternatively, patients have been administered prophylactic antibiotics in an attempt to kill bacteria before their attachment to the stent. Anti-clogging mechanical features such as side-holes (or lack of side-holes) or a unidirectional valve mechanism (to prevent influx of bacteria-laden fluids) have also been tested. To date, each of these methods has achieved mixed results not yet at the level of clinical relevance. Clearly, restenosis is a recalcitrant issue. Since prevention of restenosis cannot be assured, the patient must be monitored to ensure continued patency of the bile duct.

Current techniques for diagnosing an occluded duct - whether the occlusion is primary or due to sludge accumulation and hyperplasia - first use a blood test to monitor enzymes such as bilirubin and alkaline phosphatase, among others. An abnormal level of these enzymes can indicate a malfunctioning liver, which may be a result of the stricture 
[Joh99]. Ultrasound, computed tomography, or magnetic resonance imaging of the duct can potentially be used to confirm that a stricture exists, although often the visibility of the duct is obscured on such images. Alternatively, cholangiography - which is a procedure where contrast is injected into the biliary tree for subsequent X-ray imaging can be utilized. The blood test techniques for diagnosing a blockage are indirect and rely on detecting enzyme levels that may not increase until after the blockage is significant; additionally, the presence of the enzymes signifies liver malfunction and may be related to illnesses aside from stent occlusion. The imaging techniques are relatively expensive, require specialized equipment and operators, may be invasive, and do not allow direct visualization of the conditions inside the stent. The combined effect of the unknown time course for restenosis and the indirect testing methods can result in either unnecessary, pre-scheduled interventions or in untimely interventions after patients exhibit outward symptoms of the blockage, such as jaundice or pruritus. As such, a method of directly measuring sludge buildup would enable timely intervention and eliminate unnecessary procedures. Such a method may also add an ability to directly establish a link between the distribution of sludge and the patient prognosis.

\subsubsection{Transjugular Intrahepatic Portosystemic Shunts (TIPS)}

Portal hypertension can be defined as a large pressure gradient (12 $\mathrm{mmHg}$ or greater) along the portal vein, which drains blood from the small and large intestines, spleen, pancreas, and gallbladder. Portal hypertension is often associated with numerous liver diseases including cirrhosis, and can lead to varices (distended veins) which may 
eventually hemorrhage. Variceal hemorrhages carry a high mortality rate, so management of the portal hypertension is a critical part of managing liver disease.

To reduce portal pressure in hypertensive patients, a TIPS can be placed [Col07]. This procedure involves the percutaneous creation of a connection within the liver between the portal and systemic circulations. The procedure begins with ultrasonicallyguided access through the jugular vein to the hepatic vein. A fine needle is then used to puncture the hepatic vein. The needle is then advanced through the liver until puncturing the portal vein. A stent is then deployed across the tract to hold the shunt open. Typically, the stent is self-expanding, but may be dilated to the final desired diameter (8$12 \mathrm{~mm}$ ) with the use of an angioplastic balloon.

The most acute concern with a newly placed TIPS is hepatic encephalopathy, or the buildup of toxic substances in the blood due to impaired liver function. This can often be a short-lived situation with appropriate management of diet, although a revision of the shunt may be required. The second most common complication with TIPS placement is shunt stenosis and occlusion due to pseudointimal hyperplasia in the tract or due to intimal hyperplasia in the hepatic vein (the hyperplastic response is described in more detail in section 1.1.3). The high frequency of occlusion (33\% after 1 year and 58\% after 2 years) has led to standard surveillance with Doppler ultrasonography or portography (similar to cholangiography described in section 1.1.1 and angiography described in section 1.1.3), with a frequency of every 3-6 months after a baseline study occurring immediately after the procedure. Although the Doppler ultrasonography procedure provides a non-invasive method for determining blood flow through the shunt, criteria for shunt malfunction have proven to be difficult to determine [Dod95, Kan97]. The large 
diameters involved and relatively low fluid flows are likely contributors to this difficulty, since these two factors reduce the sensitivity of flow velocity to diameter change (this concept is further explained in section 1.2.1). As such, a non-invasive monitoring system that more directly measures buildup of tissue could also be beneficial in this application.

\subsubsection{Cardiovascular Stents}

The most common applications of cardiovascular stents are in the coronary, carotid, and renal arteries that provide circulation to the organs that use the most blood: the heart, the brain, and the kidneys, respectively. The narrowing that occurs in these arteries is due most often to the process of atherosclerosis, which is the accumulation of an atheroma ("plaque") in the sidewall of the vessel. Alternatively, the renal artery may become narrowed in hypertensive patients due to vascular smooth muscle hyperplasia (proliferation) in response to the high blood pressure.

Cardiovascular stents used to treat these constrictions are typically (if not exclusively) balloon-assisted and made out of metal (stainless steel or chrome-molybdenum). The reasons for this difference in design (compared to biliary stents) are mainly due to physician familiarity and preference: initial interventions in cardiovascular stenoses were balloon angioplasty only, making natural the addition of a balloon-assisted stent; and a stent that has an expansion that is directly controlled by the physician provides assurance in this more immediately life-threatening procedure.

Again, the primary complication in cardiovascular stent placement is restenosis. In this case, the restenosis is due mainly to neointimal hyperplasia - a proliferation of smooth muscle cells. The hyperplasia is thought to be associated with the injury response 
to the mechanical stresses exerted by the stent and delivery procedure. The production of extracellular matrix proteins by these new muscle cells also contributes to the narrowing. The hyperplasia tends to surround the stent struts and progressively occlude the lumen (Fig. 1-3). An artery may narrow by up to $70 \%$ before the outward symptoms of fatigue and chest pains are exhibited.
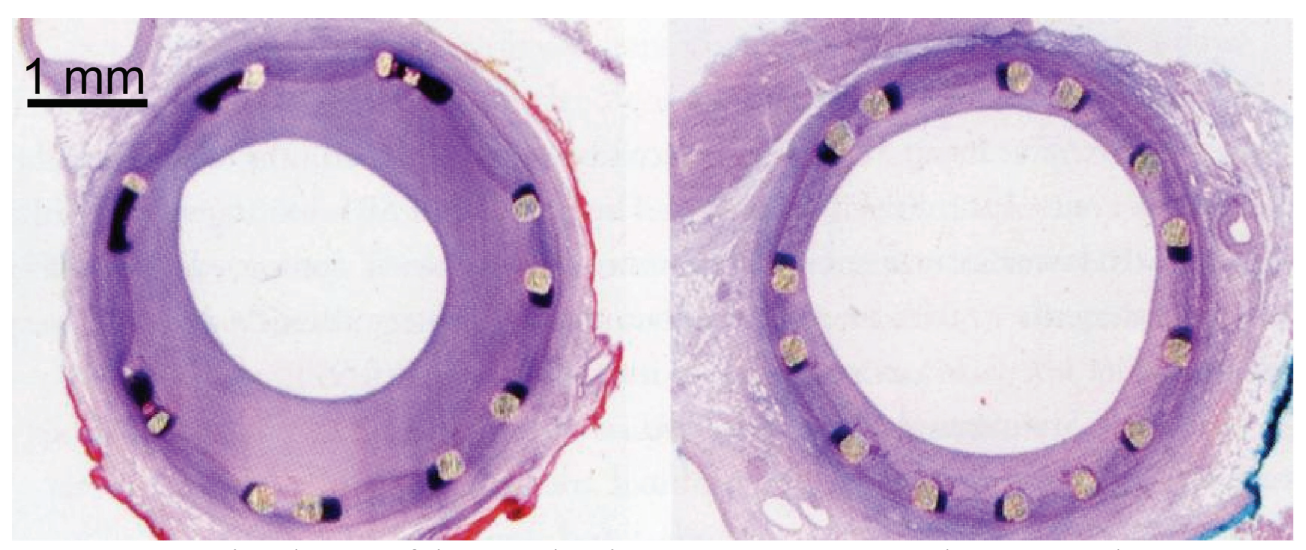

Fig. 1-3: LEFT: Histology of hyperplastic response near a bare metal stent. RIGHT: Histology of reduced hyperplastic response near a sirolimus eluting stent (from [Fax01], scale is approximate).

Recently, drug-eluting coating technology has reduced the occurrence of restenosis but the long term effects are still being studied; recent studies have shown the drug eluting stents have an increased potential for late thrombosis [Vir04, Pfi06]. Current techniques for monitoring the flow through the stent include: angiography, which consists of x-ray imaging of contrast dye injected into the blood stream; computed tomography; or magnetic resonance imaging. Alternatively, intravenous ultrasound (IVUS) or intravenous magnetic resonance (IVMR) techniques can be used. These techniques are invasive (requiring catheterization for angiography and IVUS/IVMR) and/or require specialized equipment and operators. Thus, opportunities exist for reducing invasiveness 
or expense while frequently monitoring the status of the environment of the stent [Sia04, Fax01, Ser06].

\subsubsection{Ureteral Stents}

The ureters are ducts that carry urine from the kidneys to the bladder. The ureter is wrapped with smooth muscle, and drives the flow of urine with peristaltic motion. The ureter can become constricted due to chronic inflammatory diseases, as a response to injury (e.g. from passing of a stone or surgery), or from external pressure applied to the ureteral wall (e.g. from a nearby malignancy) [Sen03, Ros07]. Ureteral stents are generally much simpler in design than other stent types discussed so far, and are either plastic (polyurethane, silicone, styrene-ethylene-butylene) tubes with periodic side holes or tightly wound metal (tantalum, nickel-titanium) coils [Hof89, Ped07]. The stent may have a pigtail on either or both ends to ensure the end of the stent stays in either the bladder or kidney as intended. In some recent studies, more difficult cases of constriction have been addressed with self-expanding metal stents that are very similar to selfexpanding biliary stents as described previously [Lia07, Bar97].

Studies have shown that, due to a pathological process similar to the formation of biliary sludge, up to $58 \%$ of indwelling ureteral stents show profuse encrustation [Tun96] - in other words, heavy formation of precipitated crystals of struvite and hydroxyapatite in a biomatrix (Fig. 1-4). Severe encrustation can lead to renal failure, hydronephrosis, and urosepsis [Whe07]. Techniques for treating this condition include replacement of the stent (although this is made difficult by embrittlement of the stent material [Tol00, Hen06]), shock wave lithotripsy (breaking up the encrustation with externally applied 
sound waves), or percutaneous laser lithotripsy [Whe07, Bul03]. Again, monitoring of the situation currently can be done with x-ray or computed tomography, with specific sonography techniques [Tri00], or with invasive cystoscopy methods. In fact, monitoring of the situation can be so difficult and progression of the encrustation so variable that web-based registries with automatic recall capabilities have been devised to eliminate the possibility of "forgotten" indwelling ureteral stents that have not been replaced according to the predetermined schedule [Lyn07]. Certainly, a monitoring technique that is inexpensive, simple, and can be frequently applied without patient discomfort would improve the quality of care in this area.

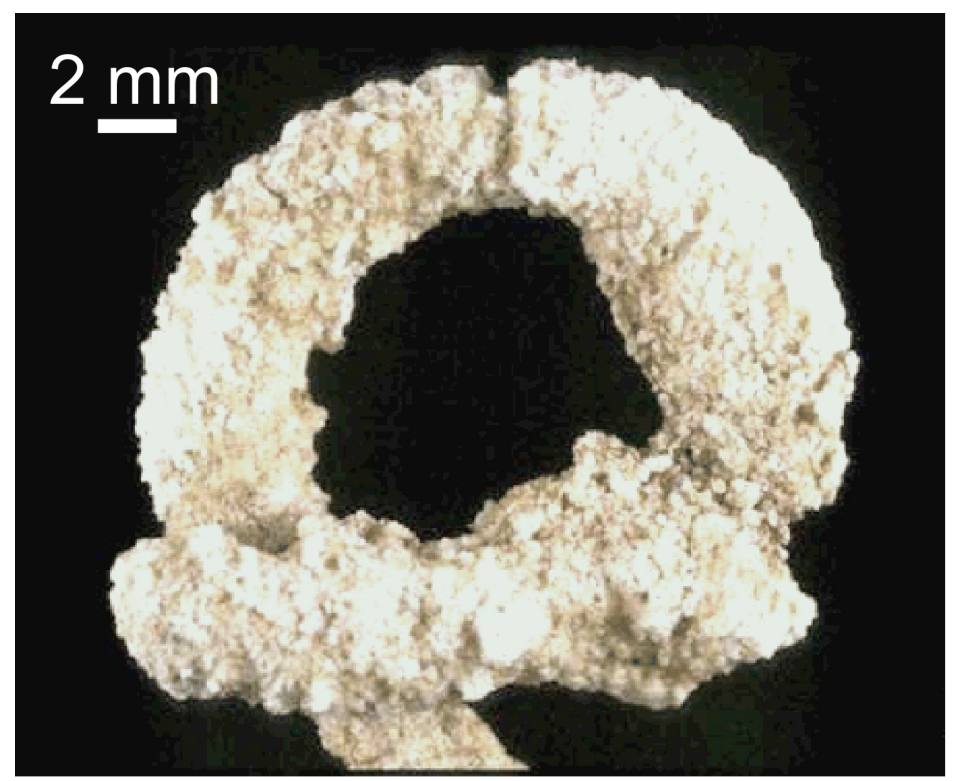

Fig. 1-4: Struvite-encrusted pigtail of a ureteral stent. Note that the encrustation forms even on the outer surface of the stent (from [Rou05], scale is approximate).

\subsubsection{Abdominal Aortic Aneurysm Stent Grafts}

The devices discussed in sections 1.1.1-1.1.3 are used to open narrow lumens. However, the attractiveness of percutaneous procedures has led to the use of stents in 
other manners. One example of this is the stent grafts that are used to treat abdominal aortic aneurysms (AAAs). An AAA is a ballooning of the aortic wall, usually located below the level of the kidneys and sometimes including one or more of the iliac artery branches. The causes of the ballooning are not clear, but genetics and hemodynamics may play a part. Aneurysms are typically asymptomatic and thus often go undiagnosed until a rupture occurs, unless the aneurysm is revealed during an abdominal examination for screening purposes or for some other medical reason. Once diagnosed, the main goal for treatment is to prevent rupture by relieving pressure on the aneurysm walls. Endovascular Aortic Repair (EVAR) is the alternative to open surgery for relieving this pressure and involves placing a stent graft in the aneurysm. This stent graft is essentially a metal (stainless steel) stent covered by a fabric (Dacron) or plastic (PTFE), and is placed such that it acts as a false lumen that does not allow blood to pass into the aneurysm. This takes pressure off the aneurysm walls and eventually the aneurysm will thrombose completely [Par95].

Unlike other stents, the most common and most threatening complication for AAA stent grafts (occurring in $2.4-45.5 \%$ of cases) is "endoleak", which refers to leakage of blood flow into the aneurysm sack through (or around) the stent graft [Mit00]. Causes of this leakage include incomplete fixation of the stent against the aorta wall outside of the aneurysm, defects of the graft material, or retrograde blood flow from other arteries. The typical method for dealing with this situation, short of open surgery, is implantation of a second stent graft that seals off the leakage in the first graft. Current techniques for monitoring for endoleak usually involve standard angiography or computed tomography assisted angiography. These methods - along with the disadvantages of high cost and 
invasiveness - do not identify all leaks. More recent work using wireless pressure sensors that are implanted in the aneurysm sack (either separately from the stent graft or integrated with the graft) show promise in identifying high pressures that may be associated with endoleaks. One such sensor (CardioMEMS, Inc.) uses a variable capacitor chamber - fashioned from metalized PTFE diaphragms or from silicon diaphragms - coupled to an inductive planar antenna. The pressure in the sack affects the capacitive gap and thus can be correlated with the resonant frequency of the inductivecapacitive tank, which can be measured using an external antenna and circuit. Another type of pressure sensor uses externally supplied ultrasound to power the device and transmit information. It is as yet unclear whether these devices have unacceptable levels of drift in long-term applications [Ohk04, Fon06, Mil04].

Another important and common AAA stent graft complication (occurring in 3-19\% of cases) is thrombosis buildup in the inner lumen of the graft. The thrombosis starts as a partial occlusion of the graft and may either spontaneously dissolve or develop into complete occlusive thrombosis [Mit00]. The cause of thrombosis is unclear, as are the reasons explaining why one thrombus collection will dissolve while another will develop. Again, thrombus formation is usually visible with angiography. However, techniques that are less invasive and less expensive could be applied more frequently and result in a better understanding of this complication as well as improve the quality of care. 


\subsection{Potential Sensor Systems for Monitoring Stents}

As is clear from the previous section, indwelling stents have significant complication rates associated with clogging due to some type of accumulation or proliferation. The complications occur within unpredictable timeframes and are currently difficult or expensive to monitor on a frequent basis. Thus, research into improved monitoring techniques is warranted.

Ideally, the monitoring system should include a sensor that can measure effects that are direct results of the sludge accumulation, smooth muscle proliferation, encrustation, or thrombosis. Specific parameters that may be important in some or all of these processes include mass accumulated on the stent, viscosity of the surrounding fluid medium, chemical or protein biomarkers associated with the process, and pressure or flow of the fluid that is being carried by the stented vessel or duct. For the monitoring system, sensitivity to one of these parameters is paramount in order to be an effective direct diagnostic technique.

Because the monitoring system is to be specifically developed for stents or stent grafts, a design that can be integrated and deployed along with the stent is desirable in order to minimize complexity of the implantation procedure. Of particular importance for implantable biomedical applications, the sensor should have the ability to be wirelessly interrogated. If the sensors can be implanted and subsequently accessed without either transcutaneous wires or catheterization, then the possibility for patient infection or discomfort is reduced dramatically. If the wireless link is simple and robust, then frequent in-home monitoring becomes a possibility, bringing with it the potential for an improved level of care. Finally, the implanted components of the system are ideally 
passive such that concerns regarding implanted battery management or energy scavenging are negated.

With the above ideal properties in mind, the following sections briefly discuss potential candidates for use as stent monitoring systems. The systems that are discussed are limited to those that have shown promise in directly measuring important parameters that can be associated with stent clogging, as well as promise for miniaturization as appropriate for integration with stents. Advantages and disadvantages of each system as they relate to stent monitoring are presented, and used to motivate further aspects of the work described in this report.

\subsubsection{Flow (Pressure) Measurement Systems}

Stents are intended to maintain patency of the treated duct or vessel so that fluid can flow, so it is natural to consider flow (or associated pressure differences) along the stent as the most important parameter to measure. This is especially pertinent in cardiovascular stents, and work in integrating pressure sensors with stents is in progress. The concept behind this measurement system lies in utilizing variable capacitance pressure sensors connected to an inductive "stentenna", thereby forming a resonant LC tank with a pressure-dependent resonant frequency that can be measured with an external coupling coil [Tak06, DeH06]. The integration of multiple resonant tanks results in the ability to measure pressure differentials along the stent which can be correlated with the flow along the stent. One particularly important aspect of this work is the efficient use of space - which is at a premium in minimally-invasive implantable devices - afforded by making the stent the working antenna for the system. This approach results in the 
possibility for much smaller wireless pressure sensor systems compared to those mentioned previously for AAAs, thus increasing the potential for integration with small stents. This approach may also be prudently applied to save space in other measurement systems as discussed later. The main issue with this approach currently is the poor inductive capabilities of demonstrated stentenna designs, resulting in tanks with very low coupling (demonstrated wireless range of $<1 \mathrm{~cm}$ ) and low quality factors that limit sensitivity. Work is being done to improve these deficiencies.

Another method for wirelessly measuring flow in cardiovascular stents is also described in [Tak06]. This method uses Faraday's law of magnetic induction to generate a voltage across two electrodes placed on opposite sides of the stent diameter. The polarizable liquid (e.g., blood) passing through the stent also passes through an externally applied magnetic field to generate the voltage. This voltage, which is proportional to the average velocity of the blood, is used to bias a diode which acts as the capacitor in the LC tank that is formed with the stentenna.

There are two major disadvantages of these two measurement methods. The first disadvantage is the likelihood of drastic sensitivity losses due to accumulation or proliferation of the substance that will clog the stent. For instance, the pressure transferred to the membrane that is used in pressure sensors can be drastically altered by layers of accumulated cells [Ste07, Fle77], and the voltage generated on the electrodes in the electromagnetic flow sensor can be severely degraded by the added capacitance of the intervening biolayers. If the buildup that the measurement system is trying to detect cancels the efficacy of the sensing technique, then the measurement system will not work as intended. 


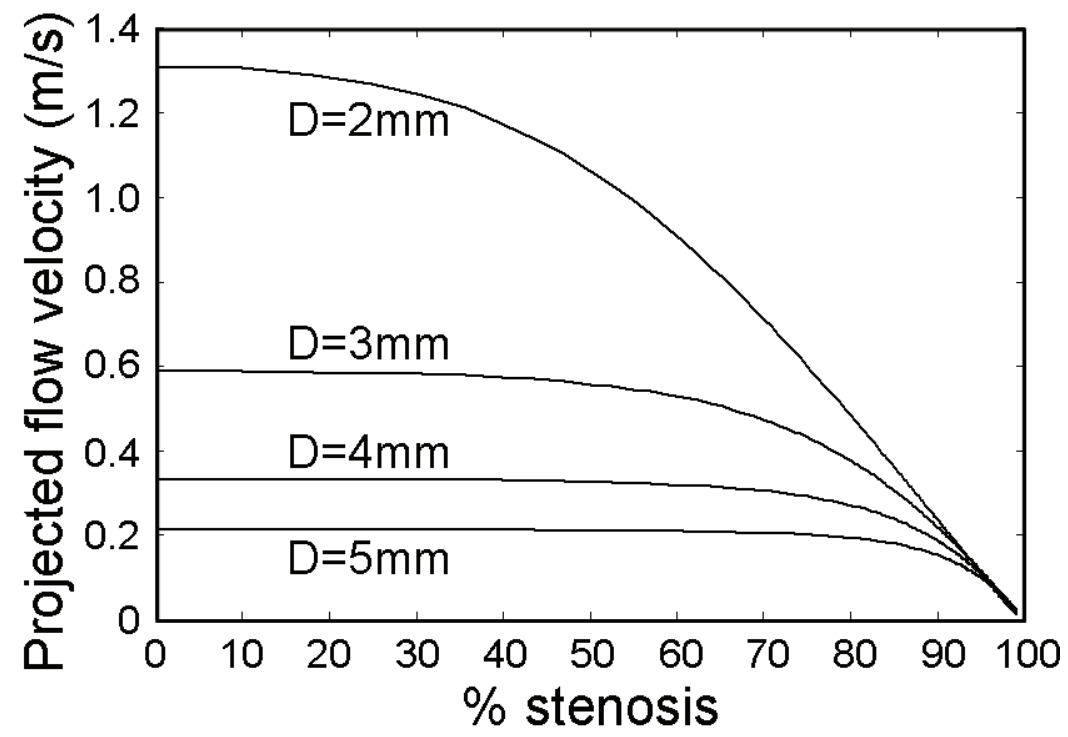

Fig. 1-5: As the diameter of the vessel or duct increases, flow velocity becomes insensitive to stenosis over a larger range (from [DeH04]). Note that the flow velocities in this chart are based on cardiovascular volumetric flow rates.

The second disadvantage of measuring pressure or flow is related to the general applicability of the measurement technique across all stent types. For coronary stents, flow rates of 100-200 $\mathrm{mL} / \mathrm{min}$ are expected, with average pressures of $\sim 100 \mathrm{mmHg}$. In contrast, flow rates in biliary stents are $3-4 \mathrm{~mL} / \mathrm{min}$ after meals and $\sim 1 \mathrm{~mL} / \mathrm{min}$ on average, while pressure differences and average pressures are on the order of 10-30 mmHg [How91, Van83], and changes in pressure and flow are less correlated with the disease state. Additionally, in large ( $>4 \mathrm{~mm})$ diameter ducts and vessels such as the bile duct, the flow rate is insensitive to duct stenosis until a substantial stenosis has occurred (Fig. 1-5). Thus, measurement of flow and pressure becomes a much more difficult task in some stent applications, even though the underlying process of clogging is rather similar in the various applications. 


\subsubsection{Electrical Impedance Measurement Systems}

Recent work has been done in sensing impedance changes between two electrodes (with one electrode sometimes being an electrolyte solution) that occur as a result of cell adhesion to one of the electrodes [Kee94, Lo95, Weg00, Mol06, Pra07]. This principle could also have application in monitoring of stents, as the adhesion of bacteria, vascular smooth muscle cells, or platelets form a central part in the restenotic pathologies described in section 1.1. In a passive wireless system, the adhesion of cells could alter the permittivity between the electrodes and thus a variable capacitor is formed. This capacitor can then be connected to a stentenna to form a resonant tank as described in section 1.2.1.

Although this technique has mainly been used with in vitro situations in which the cell culture and adhesion conditions are relatively tightly controlled, the technique may have certain advantages in implantable situations. First, the electrodes are generally simple and can easily be fabricated with simple integrated circuit processes, which generally facilitates system integration and miniaturization. The sensitivity of the technique is high, even showing potential for differentiating between single cells and their adhered shape (this assumes that the quality factor of the resonant tank is such that $\sim 1-10 \mathrm{fF}$ capacitance changes can be measured).

A disadvantage of the technique is its unproven full-scale range. Specifically, monolayer encapsulation of stents by cells is not itself a problem - it is in fact a part of normal endothelialization in cardiovascular stents. Only when the monolayer proliferates, or when it starts to generate an appreciable biomatrix, does the stent start to become occluded to the point of clinical relevance. Thus, it is critically important that the sensed 
parameter does not saturate with only a small layer of biological components. It is unclear whether an electrical impedance technique would saturate with an equivalent load of more than one adhered monolayer.

\subsubsection{Thermal Capacitance Measurement Systems}

As sludge, smooth muscle, or thrombus builds up on the stent, it is possible that the overall thermal capacitance of the system would increase proportionally to the amount of buildup. If a small resistive heater and thermistor were to be integrated with the stent, the thermal capacitance near the heater could be measured in a manner similar to that described in [Kim97]. By connecting the electro-thermal capacitor to the inductive antenna stent, a resonant LC tank with a variable capacitor can again be formed.

Again, integrated circuit processes can be used to fabricate the electrothermal sensor for this technique, with the associated benefits in system integration and miniaturization. Possible disadvantages of this technique are the need to wirelessly generate sufficient power to run the heater, as well as the unknown effects of elevated temperatures on the local biology. Additionally, water is a principal constituent of most cells, biofilms, and bodily fluids, so the thermal capacitance difference between the fluids and the buildup may be too small to sense reliably.

\subsubsection{Viscosity, Mass and Stiffness Measurement Systems}

Although viscosity, mass and stiffness are distinctly different parameters, they are presented here in one section due to the similarity in methods that have been used to measure them. The broad category of resonant sensors, consisting of mechanical 
structures that either vibrate at their resonant frequencies or are used as acoustic delay lines, have been repeatedly applied to sense changes in mass, stiffness or damping of the nearby environment [Kim05, Fer96, Nom98, Zha04]. Quartz crystal microbalances, surface and bulk acoustic wave devices, and cantilever resonators - among others - fall into this category. The basic principle behind these devices takes advantage of the coupling of the measurand to the resonant frequency of the device. For instance, changes in viscosity of fluids on the surface of a resonator will affect the damping of the resonator, while buildup of a film can contribute to changes in mass and stiffness of the system depending on the properties of the film. Additionally, the surface of the resonator can be coated with a layer that selectively binds to or is affected by targeted chemicals or biomarkers, thus potentially increasing the selectivity of the sensor as desired for specific applications.

Numerous methods of exciting the mechanical vibrations required in resonant sensors have been applied. Most popular are electrostatic drives (comb fingers or parallel plate configurations) and piezoelectric drives (usually interdigitated electrodes). The sensor can be made wireless by appropriate incorporation of an antenna and external demodulating schemes [Rei96, Bin00, Bu101]. Resonant sensors are desirable in stent monitoring applications because the measurand of interest - namely, accumulation of cells and their extracellular matrix - directly influences the frequency response of the sensor due to mass, damping, and stiffness effects of the accumulating layer. This is in contrast to pressure measurement systems where the accumulation affects flow which then is measured as pressure changes. 
Another class of resonant sensors, known as magnetoelastic sensors, utilizes an externally applied alternating magnetic field to drive the mechanical vibrations. Likewise, the mechanical vibrations affect the magnetic flux in the sensor and, as such, can be picked up with an external coil. In essence, the sensor itself acts as its own antenna and is an inherently wireless system. For stent monitoring applications, this inherent wireless capability offers a huge potential advantage, as the antenna required in all other systems along with the associated large sizes of traditional planar coils or low coupling of the antenna stent - is not a necessity. As such, the capability for direct measurement of critical parameters in stent applications - e.g. mass buildup and local viscosity - and the inherent wireless character of magnetoelastic resonant sensors are two key advantages warranting further research. This further research is the topic of this work.

\subsection{Focus of this Work}

As described in section 1.1, progressive sludge accumulation, cell proliferation, or thrombosis formation results in significant restenosis rates in a number of different types of indwelling stents. Thus, the act of stent implantation should be accompanied by a monitoring protocol to ensure continued patency of the stent. Ideally, the monitoring technique should directly measure restenosis. The technique should also result in minimal patient discomfort and require minimal expense so that the technique can be applied frequently. As illustrated in Fig. 1-6, current monitoring techniques such as angiography, MRI, ultrasound, or blood tests tend to trade the level of direct measurement of restenosis with the level of added invasiveness or expense. The focus of 
this work is to explore a stent monitoring system that more directly measures restenosis while minimizing invasiveness and added expense.

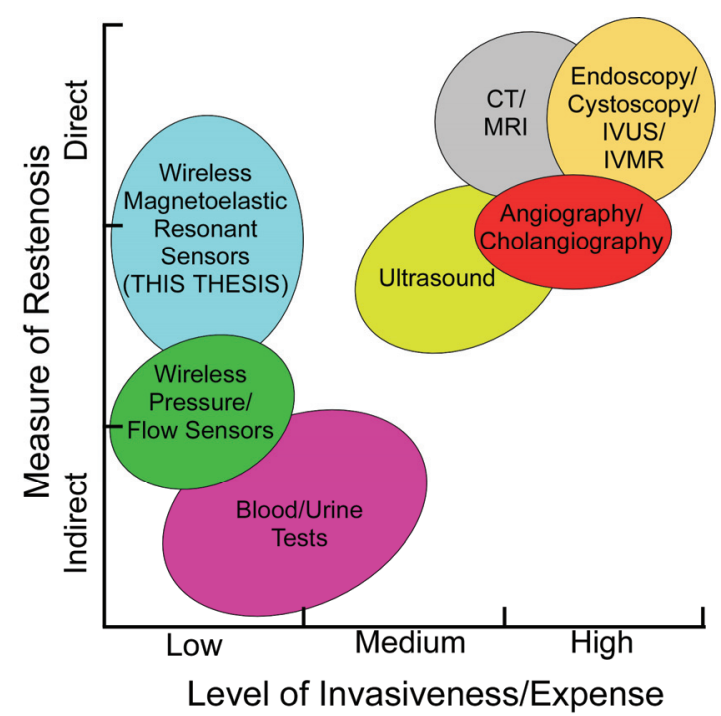

Fig: 1-6: Current diagnostic techniques trade invasiveness and expense for a direct measurement of restenosis. This work seeks to provide a technique for direct measurement of restenosis with minimal added invasiveness and cost.

As illustrated with the brief review in sections 1.2.1-1.2.4, magnetoelastic resonant sensors offer compelling properties for use in stent monitoring systems. Notably, the sensors can be used to detect important changes in viscosity and mass accumulation that are applicable to numerous stent applications as described in section 1.1. Though the general applicability of the technique is one of the major attractions, the work documented here will focus on applying the technique in biliary stent applications. Focusing the work in this way will allow detailed design specifications to be developed and addressed in order to rigorously evaluate magnetoelastic sensing as a technique for stent monitoring that minimizes invasiveness and expense while more directly measuring restenosis. 


\subsubsection{Important Past Work in Magnetoelastic Sensors}

The phenomenon of Joule magnetostriction, on which magnetoelastic transduction is based, was first described in the 1840s. More on the physics of this phenomenon will be discussed in section 2.1. All magnetic materials exhibit some amount of Joule magnetostriction; however, only starting in the early 1980's were materials with specifically tailored magnetostrictive properties developed. These materials include amorphous metals as well as the "giant" magnetostrictive Terfenol- $\mathrm{D}^{\mathrm{TM}}$ (terbium, iron, and dysprosium). The development of these materials prompted their application in sensors and actuators.

The first sensors utilizing magnetostriction were devices that measured torque, stress, and strain. The coupling between the permeability of the material and the applied strain allowed for a change in the impedance of a pickup coil as strain is induced. A review of these devices is given in [Her88]. Although the devices are wireless, all rely on very direct coupling to a ribbon sensor located directly within the coil.

The field was later expanded with the exploration of remote interrogation of magnetoelastic sensors. This work has demonstrated the feasibility of sensing mass loading, media viscosity, and other properties in environmental/industrial applications [Gri98, Sto00, Gri00, Jai01]. An early device utilizing the magnetoelastic transduction pathway consisted of NiFe films sandwiched around an analyte polymer layer [Gri98]. The polymer layer was designed to shrink or swell due to changes in the chemical environment of the sensor, and this change in sensor dimension would alter the basic shape (amplitude and width) of the induced voltage pulse train. In this example, the 
device was not used as a resonant sensor, as changes in the environment were correlated with changes in signal amplitude rather than resonant frequency.

Resonant sensors have the relative advantages of immunity to noise and changes in the amplitude or orientation of the interrogation field. Due to these advantages, later work in magnetoelastic sensors used the measured resonant frequency to correlate with properties of either the surrounding media or with properties in the sensor material or an analyte layer on the sensor. Examples of the former properties include density, viscosity [Sto00, Gri00], and pressure [Jai01] in a surrounding fluid layer, while examples of the latter properties include thermal coefficients (to measure temperature) of the sensor material or analyte layer stiffness or density (to measure the presence of a target chemical species). Ex vivo measurement of the coagulation process in small blood samples has recently been reported [Puc05]. Recent work with magnetoelastic sensors has explored acute monitoring of gastroesophageal $\mathrm{pH}$ with instrumented ingested capsules [Ong05]. In each of these cases, the stiffness, mass, or damping of the system is altered in some way, and these general parameters directly govern the frequency response. Reviews on magnetoelastic sensors have been published in 2002 [Gri02] and more recently in 2007 [Zen07].

\subsubsection{Structure of this Work}

Chapter 2 of this work presents a conceptual vision for wireless magnetoelastic monitoring of biliary stents and describes, at a general level, the key system components for achieving this vision. Subsequently, each component and the related design considerations are discussed in a more detailed manner. Of particular importance, the 
physical phenomenon of magnetoelasticity is described. Additionally, analytical and finite element design tools that have been developed for this application are described. These tools are applied to simple sensor structures - referred to as ribbon sensors throughout this work - and important results are highlighted and verified with experimental data.

Chapter 3 presents the design, fabrication and testing of a first-generation integrated wireless magnetoelastic biliary stent system. This generation incorporates simple ribbon sensors and discrete permanent magnets. This simple design provides proof-of-concept results that motivate further work.

Chapters 4 and 5 shift the focus away from simple sensor designs and explore the potential benefits of sensors that have been patterned with more complex geometry. Chapter 4 presents the design, fabrication, and testing of a second-generation integrated wireless magnetoelastic biliary stent system. This second-generation system incorporates a wishbone-array sensor that possesses an open, mesh-like structure. The system also utilizes a conformal magnetic layer that is distributed uniformly on the stent struts. Both of these enhancements result in added mechanical flexibility and expandability of the system. Chapter 5 illustrates advanced sensor designs that make use of tailored mode shapes and mechanical structures for not only improved mechanical properties but also improved monitoring functionality.

Chapter 6 discusses in situ and ex vivo testing of an integrated system that combines a simple ribbon sensor and a conformal magnetic layer with a commonly used commercially available self-expanding stent. In situ and ex vivo testing allows for a number of questions regarding the suitability of the design in its end-application to be 
answered. Finally, Chapter 7 presents a summary of the performance achieved in this work and highlights the specific contributions of this work to the field, concluding with a look toward possible future design and testing improvements. 


\section{Chapter 2}

\section{Theory, Modeling, General Design Considerations, and Component Specifications}

As noted in Chapter 1, a direct method of diagnosing biliary sludge accumulation in biliary stents would enable timely intervention and eliminate unnecessary procedures. The method outlined in Fig. 2-1, highlighting two generations of an integrated system, is a concept for providing just such a direct measurement of sludge accumulation in a biliary stent [Gre07, Gre08, Gre09]. In this concept, a network analyzer controls an amplifier, driving the transmit coils in an AC frequency sweep that produces a corresponding magnetic field sweep. The magnetic field causes a magnetoelastic sensor integrated with the stent to resonate at a frequency that changes as local viscosity increases and as sludge accumulates. The mechanical resonance generates an oscillating magnetic field that can be measured with an external pick-up coil. The frequency content of the induced voltage can then be correlated with the local sensor environment.

There are four main components of this system that warrant detailed design consideration. First and foremost, the biliary stent must be identical to currently accepted biliary stents, or at least provide comparable performance if the design is to be altered to facilitate system integration. The second component of the system is the sensor which relies on magnetoelastic transduction. The third component of the system applies the DC 
magnetic bias that is used to set the operating point of the sensor response. The fourth component of the system is the external interrogation setup, consisting of transmit and receive inductive coils, as well as the amplifier required to drive them with the modest power output of a network analyzer. Each of these components will be described in more detail in the following sections. At the conclusion of this chapter, specific performance goals will be presented on a component-by-component basis.

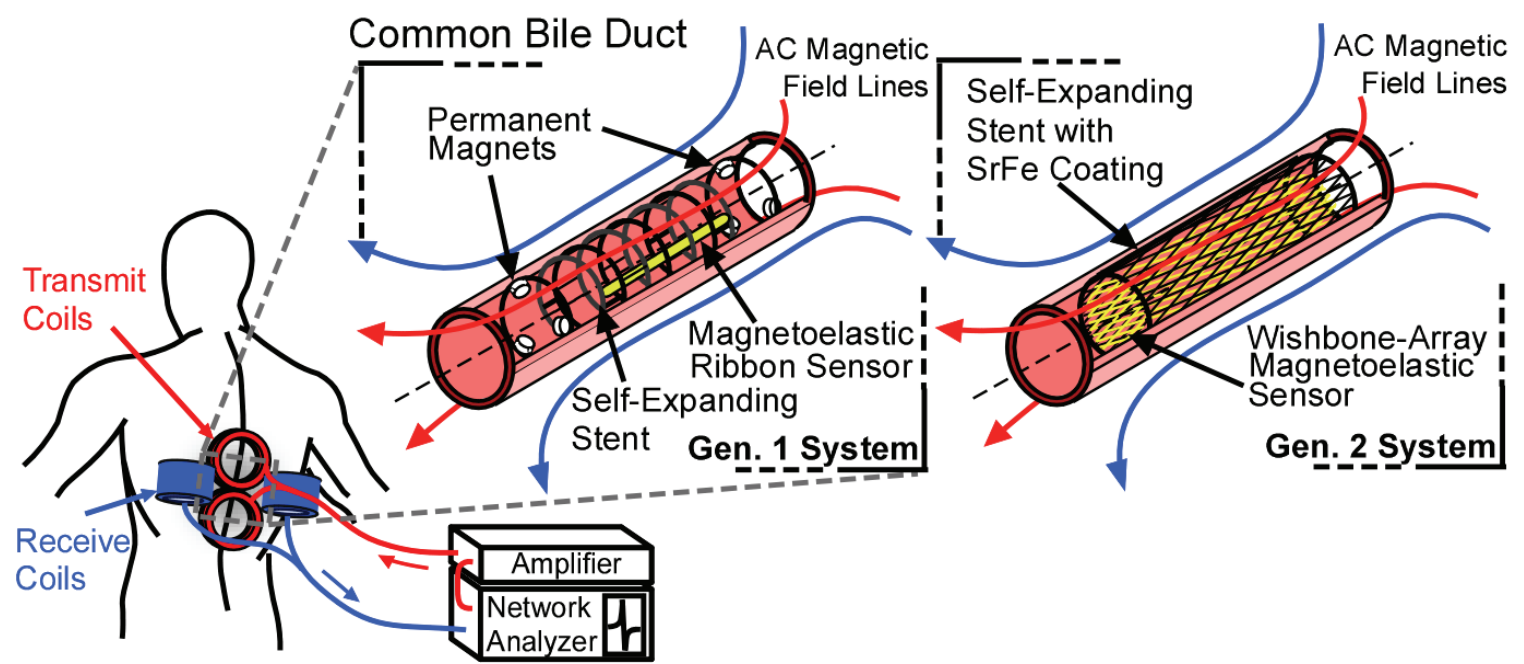

Fig. 2-1: Conceptual diagram of two generations of in-vivo magnetoelastic sensing of sludge accumulation for biliary stents. A network analyzer controls an amplifier, driving the transmit coils. The sensor, which is integrated along the sidewalls of the stent, resonates at a mass-loading-dependent frequency which is detected by the receive coils. In the Generation 1 system (Chapter 3), the discrete neodymium magnets bias the ribbon sensor. In the Generation 2 system (Chapter 4), the distributed SrFe-PDMS permanent magnet layer biases the wishbone-array sensor for optimal signal.

\subsection{Biliary Stent Design}

The main objective in biliary stent design for this work is to conform to established sizes, deliverability, and radial and bending flexibility of commercially available biliary 
stents with established clinical efficacy. Due to the need to integrate multiple system components with the stent, it may be necessary to stray outside of conventional stent geometries and fabrication processes. When such unconventional measures are taken, the resulting function of the stent should still fall acceptably near that of the proven stent designs.

As noted previously, plastic and metal stents are utilized in biliary stenting applications. Much like ureteral stents, plastic biliary stents are generally simple polyethylene tubes, from $2.3 \mathrm{~mm}$ to $3.8 \mathrm{~mm}$ in diameter and from $50-180 \mathrm{~mm}$ in length [Con05, Som06, Don07]. Note that these stents are not designed to be compressed onto a very small catheter and later expanded at the site of treatment, which is in contrast to the expanding metal stents that will be explained shortly. Plastic stents may have side-holes, flaps, or other features that are used to promote flow and reduce the potential for migration of the stent after implantation.

Metal biliary stents come in a variety of patterns, sizes, and materials [Koz00, Som06]. Metal stents are typically self-expanding and made of either Nitinol ${ }^{\mathrm{TM}}$ (nickeltitanium superelastic shape memory alloy) or Elgiloy ${ }^{\mathrm{TM}}$ (chrome-nickel alloy). It is important to note, however, that balloon-assisted designs - essentially adapted from cardiovascular counterparts - made of stainless steel or chrome-molybdenum alloys also exist (Boston Scientific), though they are infrequently documented in medical literature. Expanded diameters of metal biliary stents range from $3 \mathrm{~mm}$ to $12 \mathrm{~mm}$, with most of the available designs in the $5 \mathrm{~mm}$ to $10 \mathrm{~mm}$ range. Expanded lengths of the metal stents range from $20 \mathrm{~mm}$ to $95 \mathrm{~mm}$, with the majority of designs available in the $40 \mathrm{~mm}$ to 80 $\mathrm{mm}$ range (Cook, Abbot Medical, Boston Scientific). Some metal stents are covered with 
a silicone membrane to inhibit the potential for tumor ingrowth or mucosal hyperplasia. Of course, the defining feature of expandable metal stents is the ability to be delivered with a small catheter and yet still have a large expanded diameter upon deployment. For the majority of commercially available stents, a diametral increase of approximately 200$300 \%$ (e.g. delivery diameter of $1.3 \mathrm{~mm}$ and a final diameter of $4-5 \mathrm{~mm}$ ) is customary (Fig 2-2).

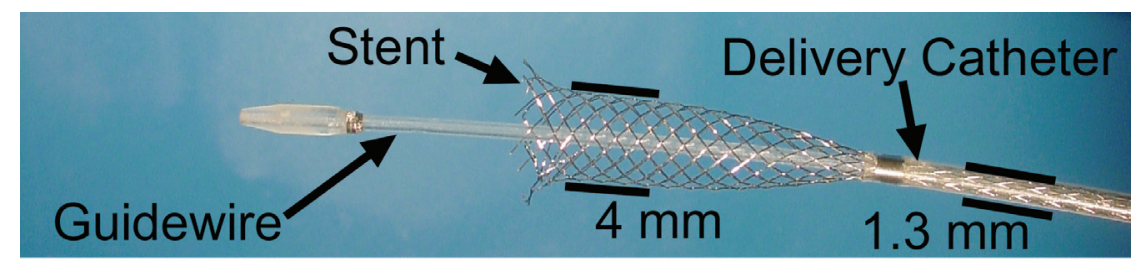

Fig. 2-2: Partially deployed, braided, self-expanding commercial biliary stent (Wallstent $^{\mathrm{TM}}$, Boston Scientific). Note the exposed wire ends.

Two main fabrication methods are used to produce metal stents. The first method consists of braiding or knitting filaments into a tubular shape. The advantage of this method as it relates to stent mechanical properties is that the filaments are not connected at the overlap points, leading to improved radial elasticity and bending flexibility. The disadvantage of this method is that the ends of the stent consist of exposed wires that may result in ulceration or perforation of the duct [Cla02]. The second fabrication method consists of cutting the desired shape from a tube of the bulk material, typically using laser machining. This method does not produce exposed wire ends; however, special attention must be paid during design to ensure that all flexural joints remain in elastic regions of strain so self-expansion can occur. Bilinear elastic-plastic finite element analysis can be 
used as a tool to evaluate the expected elastic recoil of prospective geometries prior to fabrication, as will be further discussed in Chapter 4 of this work.

As the stent is a scaffold that is to adapt to the geometry of the bile duct and prop the duct open, the radial rigidity and bending flexibility (and ability to avoid kinking in tortuous anatomy) of the stent are important properties. Reports investigating these properties are many [e.g. Cha99, Ne101, Gre04, Han05, Bas03], but each has a slightly different method for evaluating properties so direct comparisons are difficult. Additionally, most reports are not focused on biliary stents specifically but rather on general gastroesophageal stents. Thus, the reported values must be considered as general guidance only, rather than rigorous specifications. Values for radial strength and rigidity are reported in pressure until collapse ( $\sim .2$ bar), expansile stiffness per unit length for a force applied along the entire stent length with mechanical plates $(0.2-0.9 \mathrm{~N} / \mathrm{mm} / \mathrm{cm})$, and radial stiffness for a force applied with a wrap $(0.3-1 \mathrm{~N} / \mathrm{mm})$. The stiffness values agree reasonably well with a preliminary test of a $4 \mathrm{~mm}$ diameter commercial stent (Boston Scientific Wallstent ${ }^{\mathrm{TM}}$ ) subjected to local radial compression $(\sim 0.11 \mathrm{~N} / \mathrm{mm})$; the results of this preliminary test are used to establish a performance goal for designs presented in this work. Bending stiffness properties are reported in equivalent beamtheory flexural rigidity $\left(11-80 \mathrm{~N}-\mathrm{mm}^{2}\right)$, in minimum radius of curvature before onset of kinking ( 20-40 mm), and in lumen loss while in fixture tubes of various curvatures (20$25 \%$ lumen loss in $5 \mathrm{~cm}$ radius curves). An adaptation of the latter test method, as described in more detail in Chapter 4, was used to evaluate a $4 \mathrm{~mm}$ diameter by $50 \mathrm{~mm}$ long commercially available stent (Wallstent ${ }^{\mathrm{TM}}$, Boston Scientific). A 10-15\% lumen 
loss occurred for this stent when curved around a $2 \mathrm{~cm}$ standard radius. This result is used to establish a performance goal for designs in this work (see section 2.5).

For self-expanding stents, an important performance consideration is the expandability of the stent. This performance measure determines the minimum diameter of the delivery system, which is crucial in a minimally invasive therapy. Additionally, with a smaller deliverable diameter, the stent can be easily placed into very tight strictures before self-expansion. This minimizes the amount of cutting or manipulation that must be done on the stricture. As mentioned previously, commercially available designs can typically expand by $200-300 \%$; this expandability is set as a goal for designs in this work.

Further important design considerations can be gleaned from FDA guidance for the content of premarket notification for metal expandable biliary stents [FDA98]. Though this guidance does not list properties of currently cleared devices, it lists tests that should be done on both new devices and predicate devices so vital comparisons can be made. Tests include deployment testing to verify the stent is easily and accurately placed, expansion force measurements, and compression force measurements. The guidance also recommends performing each of these tests, along with visual microscopy, before and after corrosion testing to simulate the effects of prolonged exposure to gastrointestinal fluids (e.g. bile). For the purposes of this work, performance comparisons are made to predicate devices in areas that are significantly affected by design changes. 


\subsection{Sensor Design}

The following sections present important considerations regarding magnetoelastic materials and sensors, and document sensor design tools that have been developed from simplified mathematical equations describing the physical phenomenon of magnetoelasticity. Analytical and coupled finite element models are derived and key results are presented as they relate to monitoring of stents and preliminary experimental work.

\subsubsection{Physical Phenomenon and General Considerations}

Magnetoelastic transduction is a term used to describe the coupling between the classical properties of stress and strain and the quantum phenomenon of magnetism [Eng00]. Magnetoelastic behavior is most prominent in materials with strong coupling between the atomic spin moment direction and the orientation of an elongated (anisotropically shaped) electron charge cloud - so-called "spin-orbit" coupling. Then, under an applied magnetic field, the spin moments tend to rotate and align with the field, so that the macroscopic magnetization of the material is affected. The closely coupled charge cloud also rotates, and - due to the anisotropic shape of the charge cloud - the macroscopic material experiences strain. From an atomic perspective, magnetoelastic coupling describes how the alignment of the magnetic spin moments is related to the alignment of the atomic charge clouds. From a macroscopic perspective, magnetoelastic coupling describes how the magnetization response to a magnetic field is related to the strain. For magnetoelastic materials used as resonant sensors, a simplified but useful understanding of the phenomenon would result from viewing the sensor as an oscillating 
dipole magnet, with the oscillations driven by the interrogative magnetic field. The oscillating magnetic flux developed in the sensor can then induce a voltage on a suitably located pick-up coil.

Although magnetoelastic materials are generally non-linear, it is prudent and insightful to use linearized constitutive equations describing the coupling between flux, field strength, stress, and strain in a magnetostrictive material:

$$
\begin{aligned}
& \vec{\sigma}=[C] \vec{\varepsilon}-\frac{[C][d]^{T}}{\mu_{0} \mu_{r}} \vec{B} \\
& \vec{H}=-\frac{[d][C]}{\mu_{o} \mu_{r}} \vec{\varepsilon}+\frac{1}{\mu_{o} \mu_{r}} \vec{B} .
\end{aligned}
$$

Equations (1) and (2) are versions of the so-called "piezomagnetic" equations [O'De82] a name that highlights their similarity to piezoelectric equations - where $\sigma$ is the stress vector, $C$ is the stiffness matrix, $\varepsilon$ is the strain vector, $d$ is the magnetostrictivity matrix, $B$ is the magnetic flux density vector, $H$ is the field strength vector, $\mu_{o}$ is the permeability of free space, and $\mu_{r}$ is the relative permeability (assumed isotropic here). As an example, the magnetostrictivity matrix for a polycrystalline piezomagnetic material with the polar axis (direction of applied bias) in the " $\mathrm{x}_{3}$ " direction is given as [IEE71]

$$
[d]=\left[\begin{array}{cccccc}
0 & 0 & 0 & d_{14} & d_{15} & 0 \\
0 & 0 & 0 & d_{15} & -d_{14} & 0 \\
d_{31} & d_{31} & d_{33} & 0 & 0 & 0
\end{array}\right] .
$$

The rationale for using the linearized equations is analogous to that used in small-signal models of transistor-based circuits. For example, the operating point of a transistor is determined by the DC voltage applied or current supplied, whereas the operating point of a magnetoelastic resonant sensor is determined by the DC magnetic field and pre-stress. 
One important class of magnetoelastic materials is amorphous metals. Typical "transfer characteristics" relating the strain to the applied field for a given pre-stress in three grades of amorphous metals sold under the trade name Metglas ${ }^{\mathrm{TM}}[\mathrm{Met} 08]$ are shown in Fig. 2-3 (reproduced from [Cla89, Kim99, Zha06]). Note that small hysteresis effects that are present in these curves are not shown for clarity. The derivative of the curve at the chosen bias point gives the magnetostrictivity. The amorphous nature of the materials results in isotropic magnetostrictivity. Other important properties of these materials are listed in Table 2-1. Note the high permeability of these materials. The high permeability of these materials enhances the antenna-like nature of the sensor by attracting field lines and directing them along the length of the sensor, which is desirable in that the orientation of the interrogating signal is not required to be exactly along the length of the sensor for good response. However, the high permeability can also be a disadvantage, in that it limits how effectively the interrogative field can penetrate the sensor and how effectively the sensor can emit flux. In fact, both the analytical and finite element models described in sections 2.2.2 and 2.2.3 predict that the sensor signal output is inversely proportional to the fifth root of the permeability.

The choice of a bias field not only determines the small-signal magnetostrictivity, but it also determines the apparent Young's modulus of the material. The dependence of the Young's modulus on the applied field is termed the " $\Delta \mathrm{E}$ effect". The $\Delta \mathrm{E}$ effect is a direct result of the magnetomechanical coupling properties of the material [Liv82] and can be quite substantial in amorphous metals. Thus, small changes in the bias field can result in large changes in the Young's modulus. For a resonant sensor, this effect is especially important, as the resonant frequency is roughly proportional to the square root 
of the Young's modulus. As such, providing a consistent biasing field to the sensor is crucial to minimizing a potentially significant source of repeatability error. In fact, minimizing the $\Delta \mathrm{E}$ effect is the main driver for integrating the biasing magnetic components with the stent in our system, as will be further discussed in section 2.3.

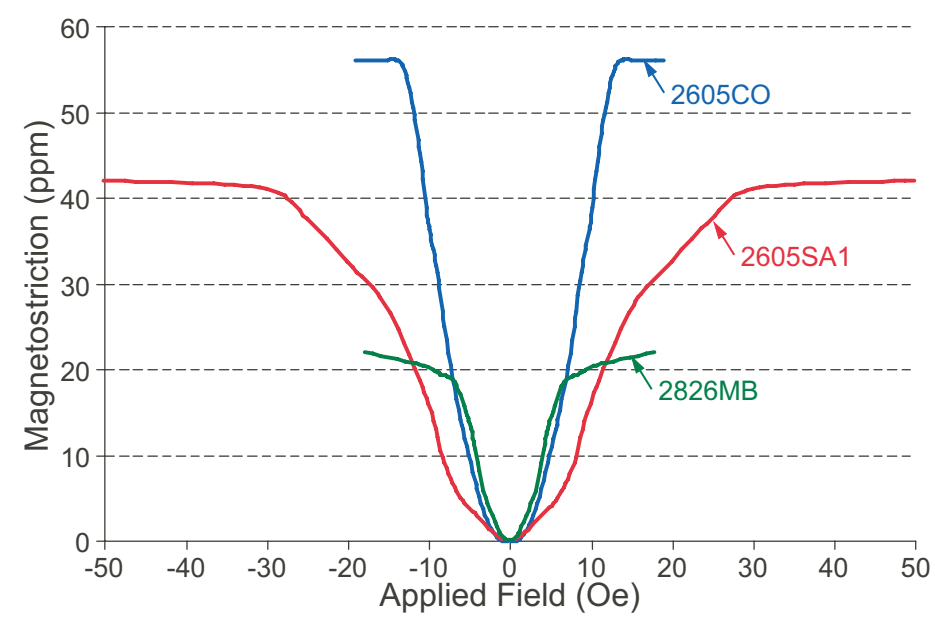

Fig. 2-3: Magnetostriction vs. applied field for various amorphous metals [Cla89, Kim99, Zha06]. The illustrated curves are from materials after annealing in a transverse magnetic field. The derivative of these curves evaluated at a bias point leads to the small-signal magnetostrictivity ("d") for the material at that bias point. These plots can be helpful for choosing between various alloys; however, they are generally established with low frequency (quasistatic) stimulating fields and thus provide incomplete information about material behavior near resonance [Kim99].

Table 2-1: As-cast material properties for various Metglas ${ }^{\mathrm{TM}}$ alloys

\begin{tabular}{|c|c|c|c|c|c|c|c|}
\hline Alloy & Composition & $\begin{array}{c}\text { Density } \\
\left(\mathbf{k g} / \mathbf{m}^{\mathbf{3}}\right)\end{array}$ & $\begin{array}{c}\text { Thickness } \\
(\boldsymbol{\mu \mathbf { m } )}\end{array}$ & $\begin{array}{c}\text { DC Relative } \\
\text { Permeability }\end{array}$ & $\begin{array}{c}\text { Saturation } \\
\text { Magnetostriction } \\
(\mathbf{p p m})\end{array}$ & $\begin{array}{c}\text { Saturation } \\
\text { Induction } \\
(\mathbf{T})\end{array}$ & $\begin{array}{c}\text { Saturation } \\
\text { Bias Field } \\
(\mathbf{O e})\end{array}$ \\
\hline $2826 \mathrm{MB}$ & $\mathrm{FeNiMoB}$ & 7900 & 29.2 & $>50000$ & 12 & 0.88 & 8 \\
\hline $2605 \mathrm{CO}$ & $\mathrm{FeCoBSi}$ & 7560 & 22.9 & 120000 & 35 & 1.8 & 12 \\
\hline 2605SA1 & $\mathrm{FeSiB}$ & 7190 & 25.4 & 45000 & 27 & 1.56 & 30 \\
\hline
\end{tabular}

It should be noted that amorphous metals are not the only materials that exhibit large magnetostriction. In fact, so-called "giant magnetostrictive materials (GMMs)" - rare earth-iron alloys like Terfenol- $\mathrm{D}^{\mathrm{TM}}$ (terbium, iron, and dysprosium) and Galfenol ${ }^{\mathrm{TM}}$ 
(gallium and iron) - have been recently explored in transducer applications (e.g. [Eng00, Zhao06]). However, when compared with amorphous metals in our application, the GMMs are less readily available in "thin" films (important for maintaining a low profile in situ). GMMs also require much larger biasing fields for optimal operation (on the order of $100 \mathrm{Oe}$ ), as well as larger alternating fields. This is due in large part to the much larger magnetic anisotropy of GMMs (not to be confused with the anisotropic shape of the atomic charge clouds) compared to amorphous materials. Preliminary experiments with Terfenol- $\mathrm{D}^{\mathrm{TM}}$ samples were unsatisfactory, although further development may prove this or a similar material advantageous in this application.

\subsubsection{Thermal Treatment of Amorphous Alloys}

Past studies of the magnetoelastic properties of amorphous metals often include annealing the material in a large transverse magnetic field (e.g. [Cla89, Kim99, Zha06, And82, Mod81]). In general, this procedure improves the magnetomechanical coupling coefficient, which is a measure of the efficiency of the material in converting magnetic energy to elastic energy and vice-versa. Transverse field annealing improves the magnetomechanical coupling coefficient by orienting the moments of the magnetic domains such that they are perpendicular to the long axis of the material. This results in nearly pure rotation of the moments when a field is applied along the long axis of the material, rather than any movement of domain walls [Spa82]. The annealing treatments can also refine the domain structure to reduce micro-eddy currents and associated losses [Her03]. The practical effects of transverse field annealing for this application include an increase in signal amplitude, along with changes in anisotropy of the material that result 
in different required biasing fields and in different structural damping exhibited by the material (evidenced by the change in quality factor of the resonant response).

To investigate the effects of transverse field annealing for each of the Metglas ${ }^{\mathrm{TM}}$ alloys (2826MB, 2605SA1, and 2605CO) with simple sensor designs, $2 \mathrm{~mm} \times 37.5 \mathrm{~mm}$ ribbon sensors were electro-discharge-machined from as-cast foils. Each sensor was then placed in a $\sim 1.5 \mathrm{kOe}$ transverse magnetic field provided between the poles of an AlNiCo major magnet and heated above the Curie temperature for the material $\left(353^{\circ} \mathrm{C}\right.$ for $2826 \mathrm{MB}, 395^{\circ} \mathrm{C}$ for $2605 \mathrm{SA} 1$, and $415^{\circ} \mathrm{C}$ for $2605 \mathrm{CO}$ ). The sensor was held at this temperature for 30 minutes and removed from the field once cooled to room temperature. The frequency response of the sensor before and after the treatment was compared. For $2826 \mathrm{MB}$, the maximum signal amplitude increased by about $10-20 \%$ after treatment, while the required bias field was reduced from 4.75 Oe to 2.25 Oe. A small increase in structural damping was also exhibited after treatment. For 2605SA1, the maximum signal amplitude increased by about $3 x$, while the required bias field was reduced from 3.75 Oe to 2 Oe. These changes were accompanied by a large increase in structural damping of the material. For $2605 \mathrm{CO}$, the maximum signal amplitude increased by about $3 \mathrm{x}$, while the required bias field increased from 2 Oe to 7.5 Oe. This material exhibited a slight decrease in structural damping after treatment. Further exploration of the transverse annealing process, including the effect of annealing time and the performance benefits for more complicated sensor geometry, is presented in Chapter 5 . 


\subsubsection{Temperature Dependent Resonant Response of Amorphous Alloys}

In this work, the magnetoelastic material is intended to be implanted. As such, the operating range for temperature will be tightly controlled, minimizing concern about temperature sensitivity of the material properties. However, to quantify such sensitivity, results from the literature are investigated. In [Jai01, Jai01_2, Jai00], Metglas ${ }^{\mathrm{TM}}$ $2826 \mathrm{MB}$ and $2605 \mathrm{SC}$ alloys are shown to have a linear resonant frequency change of $\sim 1$ $2 \%$ over the temperature range of $25-60^{\circ} \mathrm{C}$, or a temperature coefficient of about 500 $\mathrm{ppm} /{ }^{\circ} \mathrm{C}$. With the range of temperatures expected in this application, along with the mass loading and viscosity sensitivities reported in the remainder of this work, the temperature sensitivity of the material can be considered negligible.

\subsubsection{Longitudinal Magnetoelastic Ribbon Vibration and Effects of Environment}

In the proposed application, the magnetoelastic sensor is affected by the dynamic biological environment. Specifically, the surrounding liquid medium and sludge accumulation have direct effects on the response of the sensor, in terms of both resonant frequency and signal amplitude. To better quantify these effects and to provide insight into the operation of the sensor, an analytical model is proposed. This section briefly illustrates the derivation of the model; Appendix A contains a complete derivation with intermediate steps. For the following study, the sensor is assumed to be a ribbon (i.e. rectangular in shape) and the length is assumed to be along the x-axis. Also, the sensor is assumed to be fixed only at the exact mid-length, resulting in free-free end conditions.

Following a treatment in [Eng00], equations (1) and (2) can be made one-dimensional by replacing the magnetostrictivity and stiffness matrices with the isotropic 
magnetostrictivity and apparent Young's modulus (after factoring in the $\Delta \mathrm{E}$ effect of the bias field), respectively. Newton's Law is then applied to an infinitesimal volume of the sensor loaded on both sides with sludge and vibrating in a viscous medium. The strain in the sludge is assumed to be the same as that in the sensor. The analysis results in the following equation of motion relating the input magnetic field to the sensor displacement:

$$
\begin{aligned}
& {[\underbrace{\left.\left[E_{\text {app }}-\frac{E_{\text {app }}^{2} d^{2}}{\mu_{0} \mu_{r}}\right) t_{\text {sens }}+2 E_{\text {sludge }}^{*} t_{\text {sludge }}\right]}] \frac{\partial^{2} u}{\partial x^{2}}-\underbrace{\left(\rho_{\text {sens }} t_{\text {sens }}+\frac{1}{2} \rho_{f l} \sqrt{\frac{2 v}{\omega}}+2 \rho_{\text {sludge }} t_{\text {sludge }}\right)} \frac{\partial^{2} u}{\partial t^{2}}} \\
& \text { Stiffness Terms } \\
& \text { Mass Loading Terms } \\
& -\underbrace{\left(2 \mu \sqrt{\frac{\omega}{v}}+\alpha \rho_{\text {sens }} t_{\text {sens }}\right)}_{\text {Viscous Damping Terms }} \frac{\partial u}{\partial t}+\underbrace{\left(\beta E_{\text {app }} t_{\text {sens }}\right)}_{\begin{array}{c}
\text { Hysteretic } \\
\text { Damping Term }
\end{array}} \frac{\partial^{3} u}{\partial x^{2} \partial t}=\underbrace{E_{\text {app }} d t_{\text {sens }} \cdot H \cdot e^{i \omega t} \frac{d \varphi}{d x}}_{\text {Driving Term }}
\end{aligned}
$$

The derivation of specific terms is described in more detail subsequently. In equation (4), the parameters are defined as in Table 2-2.

Table 2-2: Definition of parameters in equation (4)

\begin{tabular}{|l|l|}
\hline$E_{a p p}=$ Apparent Young's Modulus & $t_{\text {sens }}=$ Sensor Thickness \\
\hline$v=$ Fluid Kinematic Viscosity & $t_{\text {sludge }}=$ Sludge Thickness \\
\hline$u=$ Local Sensor Displacement & $d=$ Magnetostrictivity \\
\hline$\omega=$ Input Signal Radial Frequency & $\rho_{\text {sens }}=$ Sensor Density \\
\hline$\varphi=$ Shape Function & $\rho_{f l}=$ Fluid Density \\
\hline$E_{\text {sludge }}^{*}=$ Sludge Complex Modulus & $\rho_{\text {sludge }}=$ Sludge Density \\
\hline$\alpha=$ Sensor Mass Damping Factor & $\beta=$ Sensor Stiffness Damping Factor \\
\hline$\mu_{o}=$ Permeability of Free Space & $\mu_{r}=$ Sensor Relative Permeability \\
\hline$\mu=$ Fluid Dynamic Viscosity & $H=$ Input Field Strength \\
\hline
\end{tabular}

The apparent stiffness of the sensor is modified by the coupling between the strain and the magnetic field as described in equations (1) and (2). The sludge is assumed to be a viscoelastic material, such that it can be described with a complex modulus possessing 
a storage aspect in-phase with the displacement and a loss aspect out-of-phase with the displacement [Sno68].

The first and third mass loading terms are simply the mass per unit length of the sensor and sludge, respectively. This assumes a uniform layer of sludge on both sides of the sensor. The second term is an effective mass loading provided by the surrounding viscous fluid, as described in [Dar01]. Briefly, the sensor (or sludge) surface and fluid interact, and a certain amount of the fluid - with a characteristic length dependent on the activation frequency and fluid viscosity - is activated and contributes to the kinetic energy of the vibration.

The first viscous damping term reflects the damping mechanism provided by the surrounding medium due to viscous shear stresses, an effect also described in [Dar01]. The second viscous damping term represents damping that is proportional to the mass of the sensor. The hysteretic damping term represents damping that is proportional to the stiffness of the sensor. The choice of modeling the overall structural damping of the sensor with terms proportional to the mass and stiffness of the sensor is due to the direct comparison that can be made to the damping assigned in the FEA model (described in section 2.2.3). Finite element modeling is at its core an algorithm for establishing and solving a matrix-based system of equations. Assigning a damping matrix that is a linear combination of the stiffness and mass matrices allows a simultaneous diagonalization of the matrices and decouples the system into single degree-of-freedom systems, speeding the solution of the system [Ang02]. Because proportional damping is thus well-suited to FEA modeling, it is also used in the analytical model for simplicity and consistency. 
The coupling described by equations (1) and (2) gives rise to the driving term. For this analysis, the interrogative magnetic field $H$ is assumed to be sinusoidal in time and uniform in space, barring the presence of the sensor. However, because the sensor has a large relative permeability, the actual field within the sensor is not uniform along the length of the sensor. The distribution of the field within the sensor is described by the shape function $\varphi$. The shape function is dependent on permeability and on sensor aspect ratio and is determined for the purposes of this analysis by fitting an equation of the form

$$
\varphi=\frac{A \cosh (B)-A \cosh \left(B \frac{x}{L}\right)}{\cosh (B)-1}
$$

to results determined from a magnetostatic finite element analysis with the desired sensor geometry and permeability. In (5), $L$ is the half-length of the sensor.

The equation of motion and associated boundary conditions can be solved via the method of eigenfunction expansion as described in [Sno68]. The result is an infinite summation of eigenfunctions multiplied by a term related to the mass loading terms, damping terms, stiffness terms, and applied magnetic field. Because we are generally concerned with only the first resonant frequency of the system, a one-term approximation is appropriate and justified according to a truncation analysis.

The one-term approximation is as follows:

$$
u(x, t)=-\frac{E_{\text {app }} d t_{\text {sens }} H \frac{2}{L}\left[\int_{0}^{L} \frac{d \varphi}{d x} \sin \left(\frac{\pi x}{2 L}\right) d x\right] e^{i \omega t}}{\left(\frac{\pi}{2 L}\right)^{2}\left(k_{\text {eff }}\right)-\omega^{2}\left(m_{\text {eff }}\right)+i \omega\left[c_{\text {visc }, \text { eff }}+\left(\frac{\pi}{2 L}\right)^{2} c_{\text {hyst }, \text { eff }}\right]} \sin \left(\frac{\pi x}{2 L}\right)
$$

where 


$$
\begin{gathered}
k_{\text {eff }}=\left(E_{\text {app }}-\frac{E_{\text {app }}^{2} d^{2}}{\mu_{0} \mu_{r}}\right) t_{\text {sens }}+2 E_{\text {sludge }}^{*} t_{\text {sludge }}, \\
m_{\text {eff }}=\rho_{\text {sens }} t_{\text {sens }}+\frac{1}{2} \rho_{f l} \sqrt{\frac{2 v}{\omega}}+2 \rho_{\text {sludge }} t_{\text {sludge }}, \\
c_{\text {visc,eff }}=2 \mu \sqrt{\frac{\omega}{v}}+\alpha \rho_{\text {sens }} t_{\text {sens }}, \\
c_{\text {hyst }, \text { eff }}=\beta E_{\text {app }} t_{\text {sens }},
\end{gathered}
$$

and $L$ is the half-length of the sensor, while $x$ is measured from the mid-length of the sensor. For the following analyses, sensor material properties used are those finalized by fitting finite element analysis results to experimental data as will be described in section 2.2.3. Although list properties for magnetostrictivity, permeability and stiffness can be applied with good results, there are no known documented values for the proportional damping values of the sensor materials, and these values play a crucial role in sensor response near resonance. Calculated results for various loads on a $2 \mathrm{~mm} \times 37.5 \mathrm{~mm}$ 2826MB ribbon sensor are shown in Fig. 2-4A. 


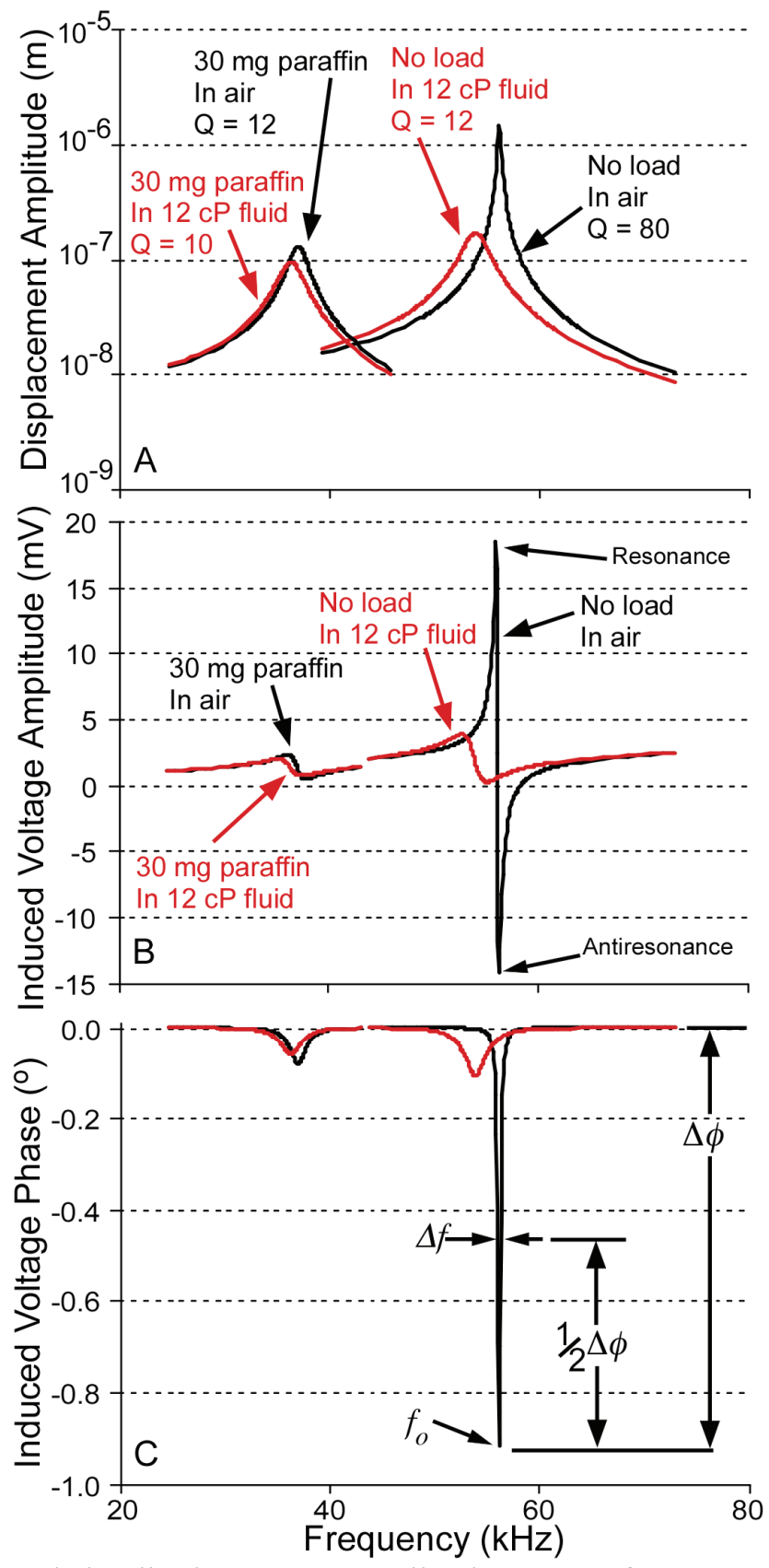

Fig. 2-4: A) Calculated tip displacement amplitude versus frequency for a $2 \mathrm{~mm} \times 37.5$ $\mathrm{mm} 2826 \mathrm{MB}$ sensor exposed to the listed environments, using equation (6). B) Calculated induced voltage amplitude - after subtracting common mode - versus frequency, using equation (14). C) Calculated induced voltage phase - after subtracting common mode - versus frequency, using equation (14). 
The mechanical vibration of the sensor is an important part of the magnetoelastic transduction effect, but the resonant response of the sensor is determined by the voltage induced on a suitably located pickup coil. To evaluate the induced voltage, the result for local sensor displacement can be back-substituted in equation (6) and the magnetic flux density in the sensor $B(x, t)$ can be solved for by substituting the right-hand side of the one-dimensional equation (2) (after differentiation with respect to $x$ ) for $H \cdot e^{i \omega t} \frac{d \varphi}{d x}$ and integrating both sides with respect to $x$. Assuming a pick-up coil is wrapped perfectly around the ribbon sensor along its entire length, applying Faraday's law of induction results in an induced voltage of:

$$
\begin{gathered}
V_{\text {sens }}(t)=\sum_{n=1}^{2} \frac{4 L_{\text {coil }} N i \omega w t_{\text {sens }} H \mu_{0} \mu_{r}}{\lambda_{n} L^{2}}\left[\int_{0}^{L} \frac{d \varphi}{d x} \sin \left(\sqrt{\lambda_{n}} x\right) d x\right] e^{i \omega t} \cdot(-1)^{n+1} . \\
\frac{\lambda_{n}\left(k_{\text {eff }, B}\right)-\omega^{2}\left(m_{\text {eff }}\right)+i \omega\left[c_{\text {visc }, \text { eff }}+\lambda_{n} c_{\text {hyst }, \text { eff }}\right]}{\lambda_{n}\left(k_{\text {eff }}\right)-\omega^{2}\left(m_{\text {eff }}\right)+i \omega\left[c_{\text {visc ,eff }}+\lambda_{n} c_{\text {hyst }, \text { eff }}\right]}
\end{gathered}
$$

where

$$
\begin{aligned}
& \lambda_{n}=\left(\frac{(2 n-1) \pi}{2 L}\right)^{2} \\
& k_{\text {eff }, B}=E_{\text {app }} t_{\text {sens }}+2 E_{\text {sludge }}^{*} t_{\text {sludge }},
\end{aligned}
$$

while $N$ is the number of turns per meter in the pickup coil, $L_{\text {coil }}$ is the half-length of the pickup coil, and $w$ is the width of the sensor. Note that two terms are now included in (11); this is because the anti-resonant frequency is well-separated from the resonant frequency in some cases and is thus more accurately determined by including the second term. An example of the induced voltage versus frequency for an unloaded $2 \mathrm{~mm} \times 37.5$ 
mm $2826 \mathrm{MB}$ ribbon sensor - with a pickup coil wrapped perfectly around the sensor - is shown in Fig. 2-5.

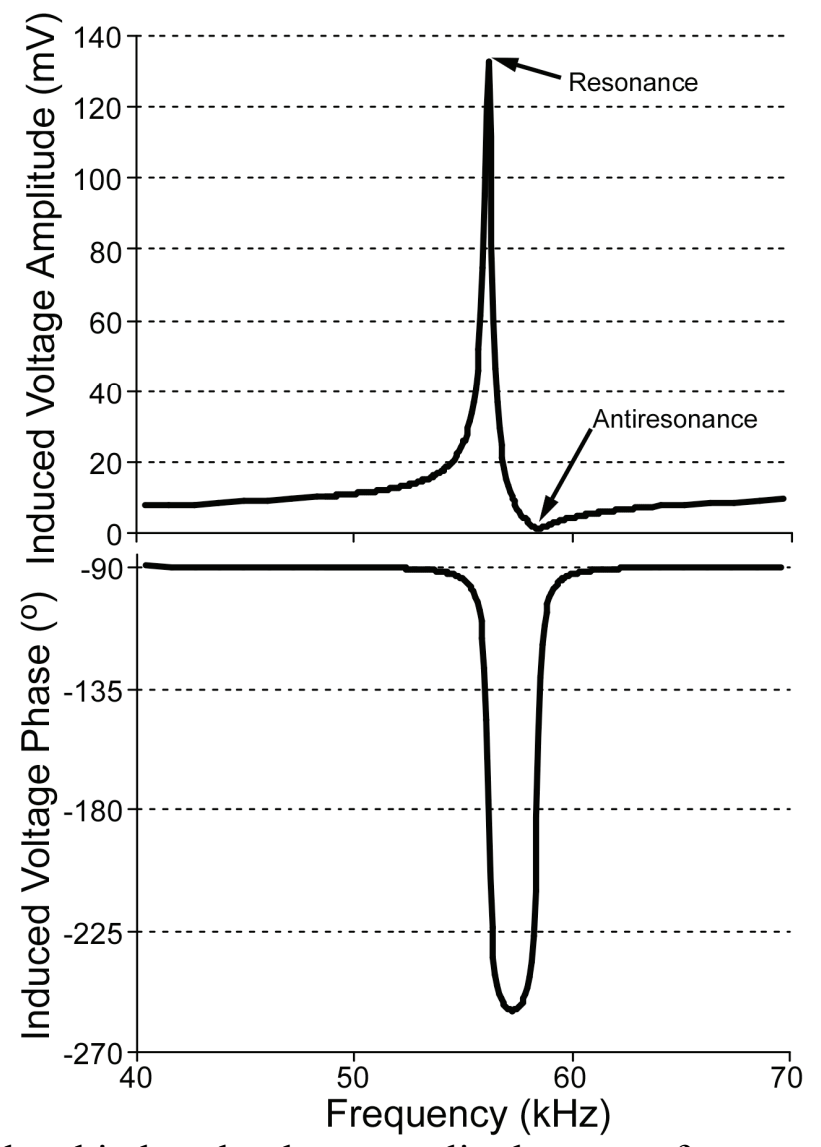

Fig. 2-5: Top: Calculated induced voltage amplitude versus frequency for a $2 \mathrm{~mm} \times 37.5$ $\mathrm{mm} 2826 \mathrm{MB}$ sensor, using equation (11). Bottom: Calculated induced voltage phase versus frequency, using equation (11). These calculations assume a pickup coil wrapped perfectly around the sensor, in contrast with the calculations displayed in Fig. 2-4B,C which assume the use of the pickup coil of configuration (A) (see section 2.4).

Equation (11) provides the voltage that would be generated in a pickup coil that is perfectly wrapped around the sensor. Although useful for providing insight into how various parameters affect the sensor operation, such an ideal pickup coil cannot be fabricated and furthermore would not be useful for wireless monitoring due to nearly zero wireless range. The effect of an actual pickup coil can be modeled by determining the 
flux that would pass through the coil as a result of a superposition of three sources: the flux in the sensor itself (as used to develop equation (11)), the flux generated by the resulting magnetization in the sensor, and the flux from the transmitted magnetic field. For instance, the voltage induced in a pickup coil that is located coaxially with the sensor can be written as:

$$
\begin{aligned}
V_{\text {coil }}(t)= & V_{\text {sens }}(t)\left(1+\frac{S}{8 L \pi w t_{\text {sens }}}\left(1-\frac{1}{\mu_{r}}\right)\right)+ \\
& \sum_{\mathrm{n}=1}^{2} N i \omega \frac{S d^{2} E_{\text {app }}{ }^{2} t_{\text {sens }} H e^{i \omega t}\left[\int_{0}^{L} \frac{d \varphi}{d x} \sin \left(\sqrt{\lambda_{n}} x\right) d x\right](-1)^{n+1}}{2 L^{2} \pi \mu_{r}\left(\lambda_{n} k_{\text {eff }}-\omega^{2}\left(m_{\text {eff }}\right)+i \omega\left[c_{\text {visc ,eff }}+\lambda_{n} c_{\text {hyst }, \text { eff }}\right]\right)}-\text { (14) } \\
& N i \omega \mu_{o} H e^{i \omega t} 2 L_{\text {coil }}\left(\pi r_{\text {coil }}{ }^{2}-w t_{\text {sens }}\right)
\end{aligned}
$$

where $S$ is the result of a triple integral that depends on the sensor and pickup coil dimensions, and $r_{\text {coil }}$ is the radius of the pickup coil. For more details on the derivation of equation (14), see Appendix A. The calculated response for a $2 \mathrm{~mm} \times 37.5 \mathrm{~mm} 2826 \mathrm{MB}$ ribbon sensor in coil configuration A (see section 2.4) for various loads is shown in Figure 2-4B,C. The induced voltage displayed in Figure $2-4 B, C$ is the shift from the baseline voltage induced in the pickup coil by only the transmitted signal (without a sensor present). Note that both the signal amplitude and the phase dip of the calculated voltage induced on a real pickup coil are much smaller than the signal amplitude and phase dip of the calculated voltage induced on an ideal pickup coil. Additionally, the locations of the calculated resonant and anti-resonant frequencies are shifted as a result of the interactions between the sensor and the coil. Fortunately, the shift in frequencies is substantially independent of pickup coil size once the pickup coil is only slightly larger than the sensor, so in all practical cases the size and location of the pickup coil only 
affects the measured magnitude of amplitude and phase, and not the frequency characteristics of the signal.

There are three main conclusions that can be gleaned from the form of equations (11) and (14) and a parametric analysis of their results. First, both resonant and antiresonant frequencies exist, and the separation between them is due in some part to the stiffness term that is modified by the coupling between the strain and magnetic field and importantly - in part to the damping of the system. Secondly, the amplitude of induced voltage is proportional to the square of the magnetostrictivity and inversely proportional to the fifth root of permeability (assuming small variations of the parameters). These dependencies are not immediately evident from the form of equations (11) and (14), but evaluating near resonance and fitting a relationship between the permeability and the shape function teases out these relationships. See Appendix A for further details. Thirdly, the signal amplitude of an unloaded sensor can be expected to vary with length and width as illustrated in Fig. 2-6. Namely, the sensor signal can be expected to increase proportionally to the width, yet decrease proportionally to the length. Experimental data from 2605SA1 ribbon sensors of varying length and width was taken in an attempt to verify these trends. The experimentally-measured signal was found to be proportional to width, as predicted by the analytical model. However, the experimentally-measured signal was found to be proportional to the length as well, which goes against the trend predicted by the analytical model. This may be a result of eddy currents that become more of a source of loss as frequency increases. Because shorter sensors resonate at higher frequencies, the eddy current losses may begin to become 
significant in shorter sensors, negating the signal gain otherwise predicted by the model.

Future models could include effects from such losses to improve predictive accuracy.
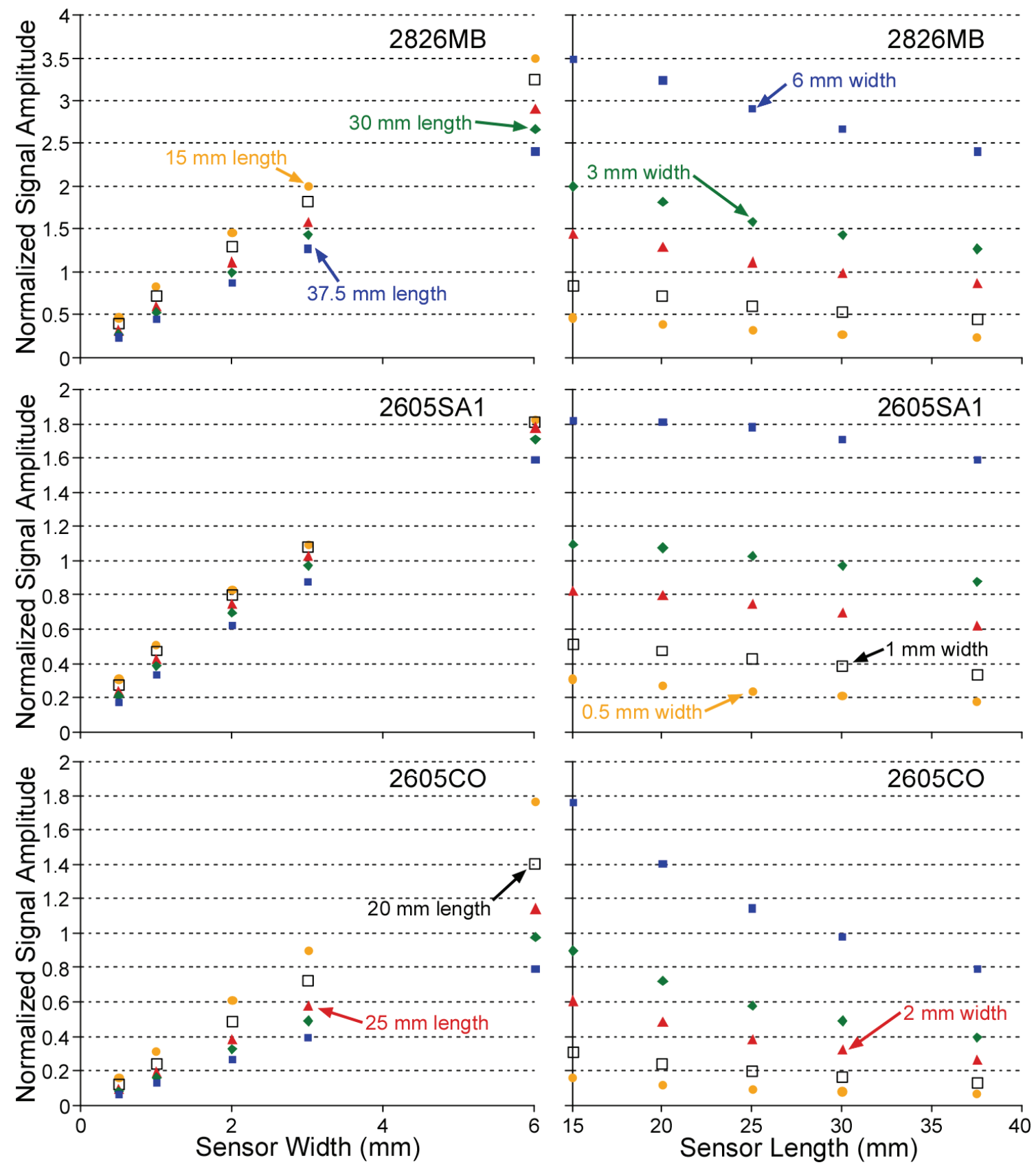

Fig. 2-6: Calculated signal amplitude variation with sensor width (left) and length (right) for three different materials: 2826MB (top), 2605SA1 (middle) and 2605CO (bottom). The amplitude for each point is normalized to the $2 \mathrm{~mm} \times 30 \mathrm{~mm} 2826 \mathrm{MB}$ calculated amplitude. In general, the signal amplitude is predicted to increase with width and decrease with length. 


\subsubsection{Material Selection for Sensing in a Viscous Environment}

The analytical model developed above can be used to gain insight into how material properties affect the robustness of the signal from the various materials in the face of viscosity and mass loads. This is an important consideration for this application because the magnetoelastic sensor must provide a usable wireless signal to the external interrogation system even under loading from the dynamic biological environment in which the sensor is deployed. In this application, the sensor will at least be fully immersed in bile, the viscosity of which will reduce the signal amplitude of the sensor.

As can be seen in comparing equations (6) and (11), the amplitude of the voltage that is induced on a pickup coil is proportional to the amplitude of the longitudinal displacement. Assuming that the damping and mass loading due to the fluid medium in which the sensor is immersed is only significant near resonance (so that the resonant frequency $\omega_{r}$ can be substituted for $\omega$ in equations (8) and (9)), and substituting the relation for the quality factor

$$
Q=\frac{\sqrt{\left(\frac{\pi}{2 L}\right)^{2} k_{\text {eff }} m_{\text {eff }}}}{c_{v i s c, e f f}},
$$

the maximum displacement can be expressed as

$$
\left|u_{\max }\left(L, \omega_{r}\right)\right|=\frac{E_{\text {app }} d t_{\text {sens }} H \frac{2}{L}\left[\int_{0}^{L} \frac{d \varphi}{d x} \sin \left(\frac{\pi x}{2 L}\right) d x\right]}{\left(\frac{\pi}{2 L}\right)^{2} k_{\text {eff }}} \cdot \frac{Q^{2}}{\sqrt{Q^{2}-\frac{3}{16}}} .
$$

It can be seen from (16) that the maximum displacement (and signal) is largest for an otherwise unloaded sensor with a large quality factor. For this application, however, the 
sensor will be loaded at least by the viscous bile surrounding the sensor, and it is important that the sensor still provides a large signal. In a viscous fluid, the ratio of the loaded displacement to the unloaded displacement reduces to

$$
\frac{\left|u_{\text {max }, \text { loaded }}\right|}{\left|u_{\text {max }, \text { unloaded }}\right|}=\frac{Q_{\text {loaded }} \sqrt[2]{Q_{\text {unloaded }}^{2}-\frac{3}{16}}}{Q_{\text {unloaded }}^{2} \sqrt{Q_{\text {loaded }}^{2}-\frac{3}{16}}} \approx \frac{Q_{\text {loaded }}}{Q_{\text {unloaded }}}(\text { assuming } Q>>1) .
$$

Although the resulting relationship in (17) is derived from the simple geometry and longitudinal mode shape of a ribbon sensor, it is valid for more complex mode shapes that can be described with effective mass, stiffness, and damping parameters. Assuming that the mass loading effects of the viscous fluid are small - a valid assumption at frequencies above a few kilohertz - then the loaded and unloaded effective mass and stiffness cancel in the ratio of quality factors in (17), leaving only the loaded and unloaded effective damping parameters:

$$
\frac{\left|u_{\text {max }, \text { loaded }}\right|}{\left|u_{\text {max, unloaded }}\right|} \approx \frac{c_{\text {visc,eff, unloaded }}}{c_{\text {visc,eff,loaded }}}=\frac{c_{\text {visc,eff, unloaded }}}{c_{\text {visc,eff, unloaded }}+c_{\text {visc, fluid }}}
$$

It can be seen in (18) that, in order to maintain signal strength even while immersed in a viscous fluid, the unloaded effective damping of the sensor should be comparable to the damping added by the viscous fluid. With a similar argument, it can be shown that viscoelastic mass loads with a significant damping factor (such as biofilms like sludge [Cen06]) can reduce the loaded amplitude even further. This analysis shows that the sensor material and geometry for this application should provide a large signal while unloaded and also exhibit damping that suitably matches the viscous loads present in the application. 
Using a material and geometry with a relatively low quality factor, as is proposed with the above analysis, is counter to the design philosophy used in most resonant sensors. A higher quality factor is associated with a sharper resonant peak, and thus a smaller minimum resolvable resonant frequency change. Along with the sensitivity of the sensor, this determines the minimum resolvable added mass. In contrast with many masssensitive resonant sensor applications, in which small amounts of mass accumulate at one binding location in an air or vacuum environment, this application requires measuring large amounts of mass that accumulates everywhere in a liquid environment. As such, the viscoelastic properties of the mass load and the viscous properties of the liquid environment dominate the quality factor of the resonant system. For this reason, improving the inherent quality factor of the unloaded resonating material is not a major point of emphasis for this application.

The ramifications of the inherent quality factor of the sensor material can be further explored by comparing expected results for two amorphous metal materials with different unloaded quality factors. In Fig. 2-7, the calculated resonant frequency and signal amplitude response to paraffin mass loads for $2 \mathrm{~mm} \times 37.5 \mathrm{~mm}$ sensors made of either Metglas $^{\mathrm{TM}} 2826 \mathrm{MB}, 2605 \mathrm{SA} 1$, or $2605 \mathrm{CO}$ is shown. The signal amplitudes are normalized to the calculated amplitude of the unloaded $2826 \mathrm{MB}$ sensor, and the resonant frequencies are normalized to the calculated unloaded resonant frequency for each sensor type. A critical result is that the signal amplitude for both the 2605SA1 and $2605 \mathrm{CO}$ sensors, although initially lower than the initial amplitude of the $2826 \mathrm{MB}$ sensor, maintains a higher level after mass loads of approximately $3 \mathrm{mg}$ and $15 \mathrm{mg}$, respectively. This result is due to better impedance matching between the load and the 2605SA1 and 
$2605 \mathrm{CO}$ sensors - the quality factor for the unloaded $2605 \mathrm{CO}$ sensor is approximately 10 and the quality factor for the $2605 \mathrm{SA} 1$ sensor is approximately 20 , while the unloaded quality factor for the $2826 \mathrm{MB}$ sensor is approximately 80 . The lower quality factors better match the low quality factor of the loading material and thus retain more of the unloaded amplitude.
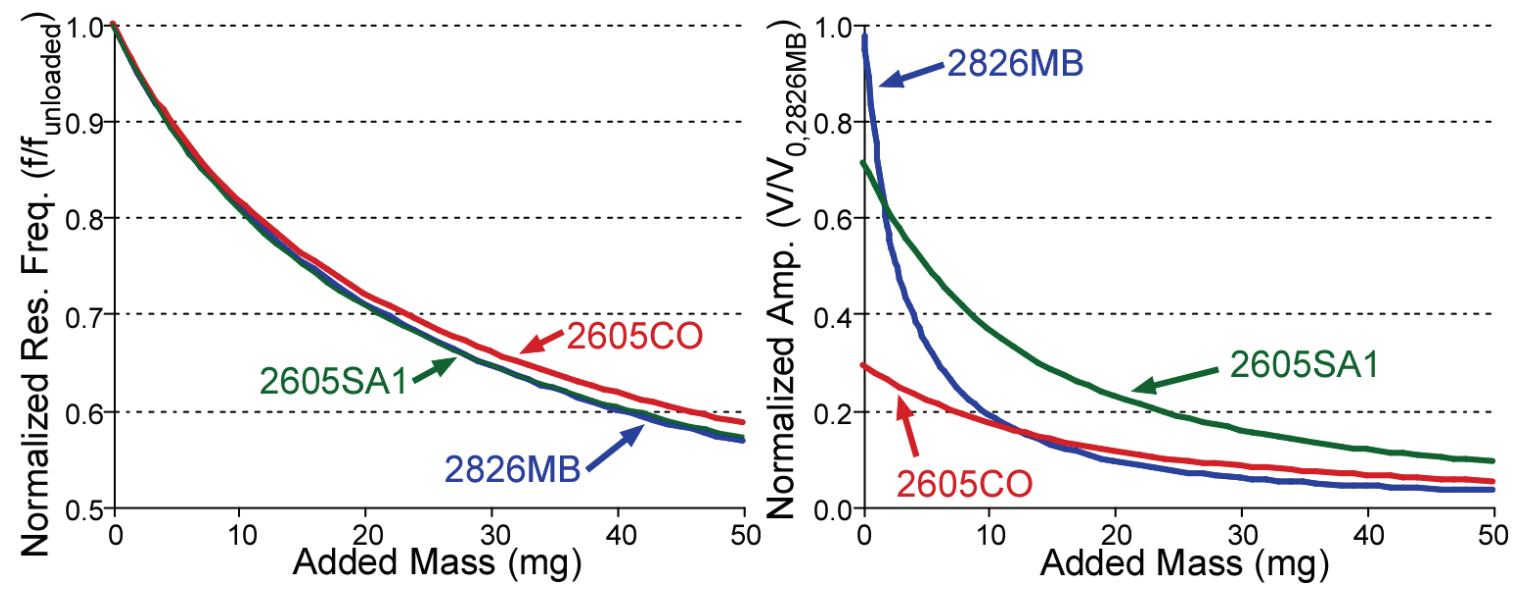

Fig. 2-7: Calculated resonant frequency and signal amplitude response to paraffin mass loading for $2 \mathrm{~mm} \times 37.5 \mathrm{~mm}$ sensors of three different materials. The resonant frequencies are normalized to the unloaded resonant frequency for each sensor type, while the signal amplitudes are normalized to the unloaded amplitude of the $2826 \mathrm{MB}$ sensor.

The simulation results in Figs. 2-4 and 2-7 use values for paraffin as a load [Sev01] rather than values for sludge. This is to facilitate comparison with experimental results in this work as described in Chapters 3 and 4 . Note that biofilms like sludge are likely to be less stiff and have a lower loss modulus than the test materials used in this work [Cen06].

To provide experimental verification of the principal described in this section, $2 \mathrm{~mm}$ x $37.5 \mathrm{~mm}$ ribbon sensors are fabricated (using serial mode micro-electro-discharge machining) from three different Metglas ${ }^{\mathrm{TM}}$ materials: $2826 \mathrm{MB}, 2605 \mathrm{CO}$, and $2605 \mathrm{SA} 1$. 
Fig. 2-8 shows the frequency and mass load response of the sensors when loaded by dipcoated paraffin. The frequency response is normalized to the unloaded resonant frequency for each sensor, and the load is normalized to the unloaded mass of each sensor. The amplitude response is not normalized to show the critical findings of this study: although the $2605 \mathrm{CO}$ and $2605 \mathrm{SA} 1$ sensors have unloaded amplitudes and quality factors that are smaller than that of the unloaded $2826 \mathrm{MB}$ sensor, the amplitudes are much less affected by the load. After an added normalized mass of approximately 2, the 2605SA1 sensor has a larger signal amplitude than a similarly loaded 2826MB sensor. After an added normalized mass of approximately 3 , the $2605 \mathrm{CO}$ sensor has a larger signal amplitude than a similarly loaded $2826 \mathrm{MB}$ sensor. Based on the data trends, the $2605 \mathrm{CO}$ material may provide the largest signal of any material at large normalized mass loads. These trends are qualitatively predicted by the analytical model, although the model tends to underestimate the absolute value of the signal amplitude at a given mass load (possibly because the moduli in the model for paraffin are slightly high).

Similarly, Fig. 2-9 shows the amplitude response of the sensors while immersed in fluids of different viscosities. Again, it can be seen that the initially large amplitude and quality factor of $2826 \mathrm{MB}$ is significantly affected by added damping in the form of increased viscosity, while the amplitudes of the materials with an initially lower quality factor are less affected by increased damping. This theme of appropriate matching of the sensor quality factor to that of the expected loads will be revisited in Chapters 5 and 7 . 


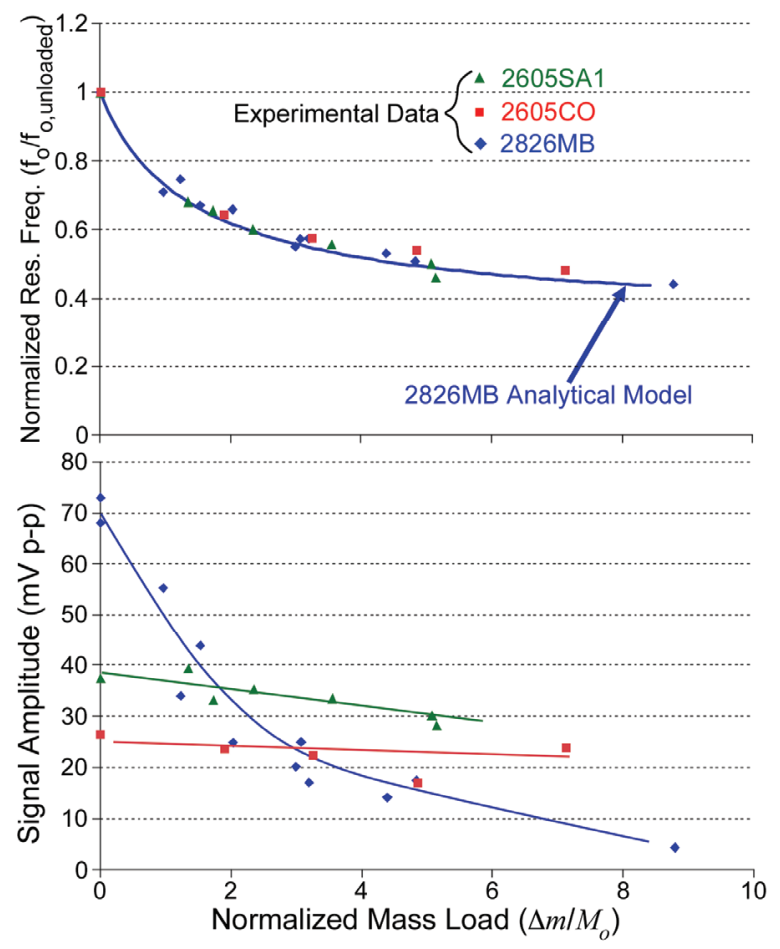

Fig. 2-8: Alternative amorphous metal materials provide similar resonant frequency sensitivity to paraffin mass loads but vastly different amplitude responses due to the differing mechanical impedance of each material. Curves in the bottom graph are guides to the eye.

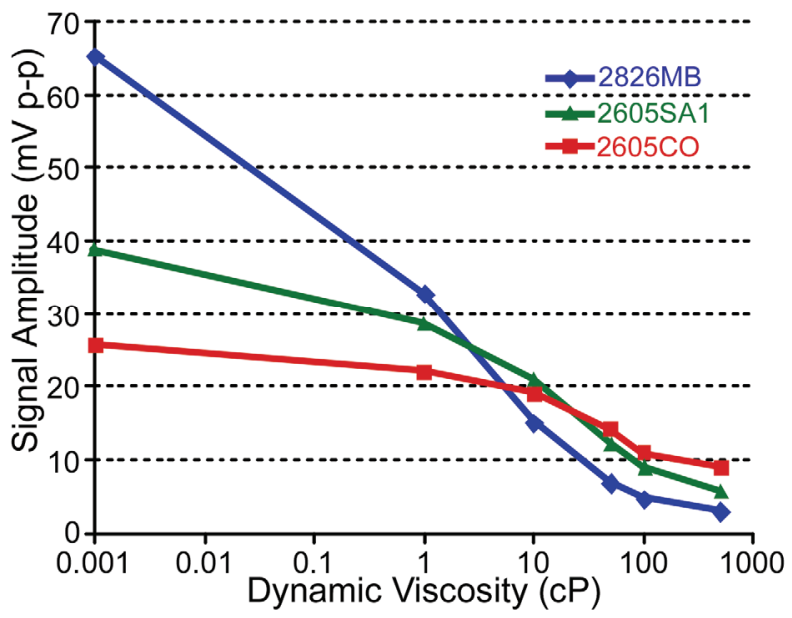

Fig. 2-9: Alternative amorphous metal materials again show vastly different amplitude responses to increasing viscosity due to the differing mechanical impedance of each material. The crossover points for amplitude occur in the $3-20 \mathrm{cP}$ range of viscosity, which is similar to values quoted in literature for bile [Jun01]. 


\subsubsection{Separation of Viscosity and Mass Response}

The analytical model shows that shifts in the resonant frequency of the sensor occur when the sensor is loaded by mass and by fluids of different viscosity. As such, the shift in resonant frequency can be used to characterize the accumulation of mass on the sensor or shifts in viscosity of the fluid in which the sensor is placed. However, ideal monitoring of the environment within the stent would include the ability to separately characterize mass accumulation from shifts in fluid viscosity. Monitoring a single parameter - resonant frequency - that is sensitive to both makes such separate characterization difficult. If two parameters in the frequency response of a single sensor can be determined that have different sensitivities to mass loads and viscosity shifts, then separate characterization can be accomplished with that single sensor. As discussed previously, shifts in viscosity represent a shift in damping and can affect the quality factor of the resonant system, with an increase in damping resulting in a reduction of the quality factor and vice versa. Meanwhile, the resonant frequency of the system has an inverse relationship to mass, while the quality factor has a direct relationship to mass. Thus, simultaneously monitoring the resonant frequency and quality factor of the system should provide the ability to separate the accumulation of mass from shifts in viscosity.

For a simple second-order resonant system, the quality factor can be determined by dividing the half-power bandwidth of the resonant peak into the resonant frequency. However, this algorithm is made more difficult for the measured wireless amplitude response of a magnetoelastic sensor due to the nearby presence of an anti-resonant frequency that distorts the shape of the resonant peak. Fortunately, the measured separation between the resonant and anti-resonant frequency is also determined by the 
quality factor of the system, so this more easily measured value can be used as a substitute. To complicate matters further, the exact location of the resonant and antiresonant frequency is often difficult to determine for a loaded sensor because the damping added by the load smears the resonant and anti-resonant peaks in the measured amplitude response. However, these difficulties can often be avoided by using the phase response of the sensor, which exhibits a characteristic trough (inverted peak) as illustrated in the calculated response shown in Fig. 2-4C. The shape (i.e. half-power bandwidth, $\Delta f$ ) and location (i.e. frequency at the minimum phase response, $f_{o}$ ) of this phase trough are related to the quality factor and resonant frequency of the system respectively, and as such can be used in combination to separate mass loads from shifts in viscosity as described presently.

Further insight into the separation of the effect of mass loads from that of viscosity shifts using these measured parameters can be had by once again investigating the analytical model for the ribbon sensor. For this analysis, equation (11) is simplified by making the following assumptions: only the first term $(n=1)$ is used; the damping due to structural hysteresis in the sensor is negligible compared to damping due to the loading material and due to the viscous medium, such that the term containing $\alpha$ in (9) and the damping term proportional to $\beta$ in (10) disappear; mass loading due to the viscous medium is negligible compared to other effective mass loads present, such that the second term in (8) disappears; damping due to viscosity is only important near resonance and anti-resonance, allowing the center frequency $\omega_{o}$ to be substituted for $\omega$ in (9); and the real part of the complex sludge modulus is negligible compared to the sensor modulus. 
Then, the added mass $(\Delta m)$ can be related to the frequency at which the minimum phase occurs, $f_{o}$, through the following relation:

$$
\frac{\Delta m}{M_{o}}=\left(\frac{f_{o, \text { unloaded }}}{f_{o}}\right)^{2}-1
$$

where $M_{o}$ is the mass of the sensor and $f_{o \text {,unloaded }}$ is the frequency at which the minimum phase occurs for an otherwise unloaded sensor. Meanwhile, the viscosity-density product of the fluid medium can be related to $\Delta f$ - the separation between the resonant and antiresonant peak (or alternatively, the half-power bandwidth of the phase trough) - and $f_{o}$ through the following relation:

$$
\sqrt{\mu \rho_{f l}}=C \frac{\Delta f}{f_{o}^{\frac{5}{2}}}+D \frac{1}{f_{o}^{\frac{3}{2}}},
$$

where $C$ and $D$ are constants dependent on sludge and sensor material properties. $C$ and $D$ can be used as fitting parameters for use in sensor calibration, as is done in the remainder of this work.

To provide a concrete example of the ability to separate mass loading from viscosity shifts, a $2 \mathrm{~mm} \times 37.5 \mathrm{~mm}$ ribbon sensor machined from Metglas ${ }^{\mathrm{TM}} 2826 \mathrm{MB}$ was dipcoated in paraffin and immersed in fluids of known properties (DI water and Dow Corning 200 fluids). As shown in Fig. 2-10, the results can be reasonably well fit using the relationships in (19) and (20) for this combination of materials and geometry (for the results shown in Fig. 2-10, $C$ takes the numerical value of $1.277 \mathrm{E} 9$, while $D$ takes the numerical value of -1.498E7). Further experimental results are investigated in Chapter 5 to evaluate the applicability of this technique with different sensor geometries and sludge simulants. 


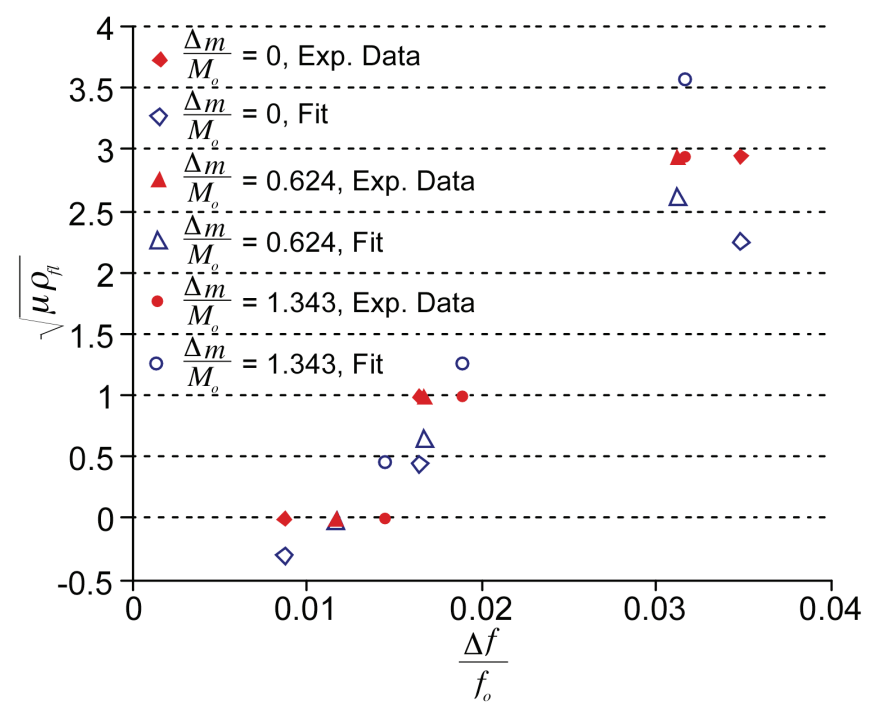

Fig. 2-10: Measuring the center frequency and resonant - anti-resonant bandwidth along with the relationships presented in equations (19) and (20) allow shifts in the viscositydensity product to be estimated separately from the effects of mass loading.

\subsubsection{Finite Element Modeling}

The above analysis provides insight into the operation of ribbon sensors, which have a simple longitudinal vibration mode shape. However, more complicated sensor geometries - such as the wishbone-array sensor pattern that is used in the secondgeneration system presented in this work (Chapter 4) - represent a significant departure from typical ribbon sensors. The analytical model cannot be used to estimate the resonant frequency or signal amplitude of such complex geometries. To address such situations, a finite element analysis (FEA) tool is developed that is appropriate for estimating mode shapes and expected signal amplitudes from sensors with complicated structures. The tool can also be used to predict signal amplitudes measured by systems with real receive coils located some distance from the sensor. The key component of this tool is the use of equations (1) and (2) to establish coupling between the magnetic and structural physics domains. For this work, the FEA code is implemented in COMSOL 
Multiphysics, with implementation facilitated by the use of weighted residual or "weak" formulations of the equations. A detailed look at an FEA implementation for magnetostrictive materials is in [Ben05]; the approach used in this work is modified for specific application to resonant sensors by utilizing time-harmonic (frequency response) structural and magnetic analysis modes.

The desired sensor geometry is modeled in the FEA program, along with the geometry of the transmit coil. If possible, the receive coil should also be modeled as a cylinder in the desired location; this facilitates the numerical integration of flux through the receive coil. For the purposes of this work, the FE code was first verified using experimental data from the coaxial coil configuration (as described in section 2.4) and 2 $\mathrm{mm} \times 37.5 \mathrm{~mm} 2826 \mathrm{MB}$ and $2605 \mathrm{CO}$ ribbon sensors. The current in the transmit coil was measured (see section 2.4) and applied in the model to generate the alternating field at the sensor. The flux from the sensor response is integrated numerically over the volume of the receive coil to establish the induced voltage. The frequency of the current is swept over an appropriate range, and in this manner the voltage frequency response of the system can be calculated and compared to experimentally obtained data. Values available in the literature for apparent modulus, permeability, and magnetostrictivity of the material are used, along with reasonable values for proportional damping. Small modifications to the literature values for the material parameters result in excellent fit with experimental data as shown in Fig. 2-11. See Appendix A for more details on the exact parameters used. Note that amplitude and phase information can be used to characterize the frequency response of the sensor, and in certain loading situations a better signal-to-noise ratio may be obtained by viewing phase information rather than 
amplitude information or vice versa.

As done with the analytical model, the effect of aspect ratio on the signal amplitude for unloaded ribbon sensors was investigated, with results as shown in Fig. 2-12. As with the analytical model, the signal amplitude increases as the sensor width increases. The variation with length predicted by the FEA is more complex than the linear decrease in amplitude as length increases predicted by the analytical model; however, in general the trend predicted by the FEA is again a decrease in amplitude as length increases.
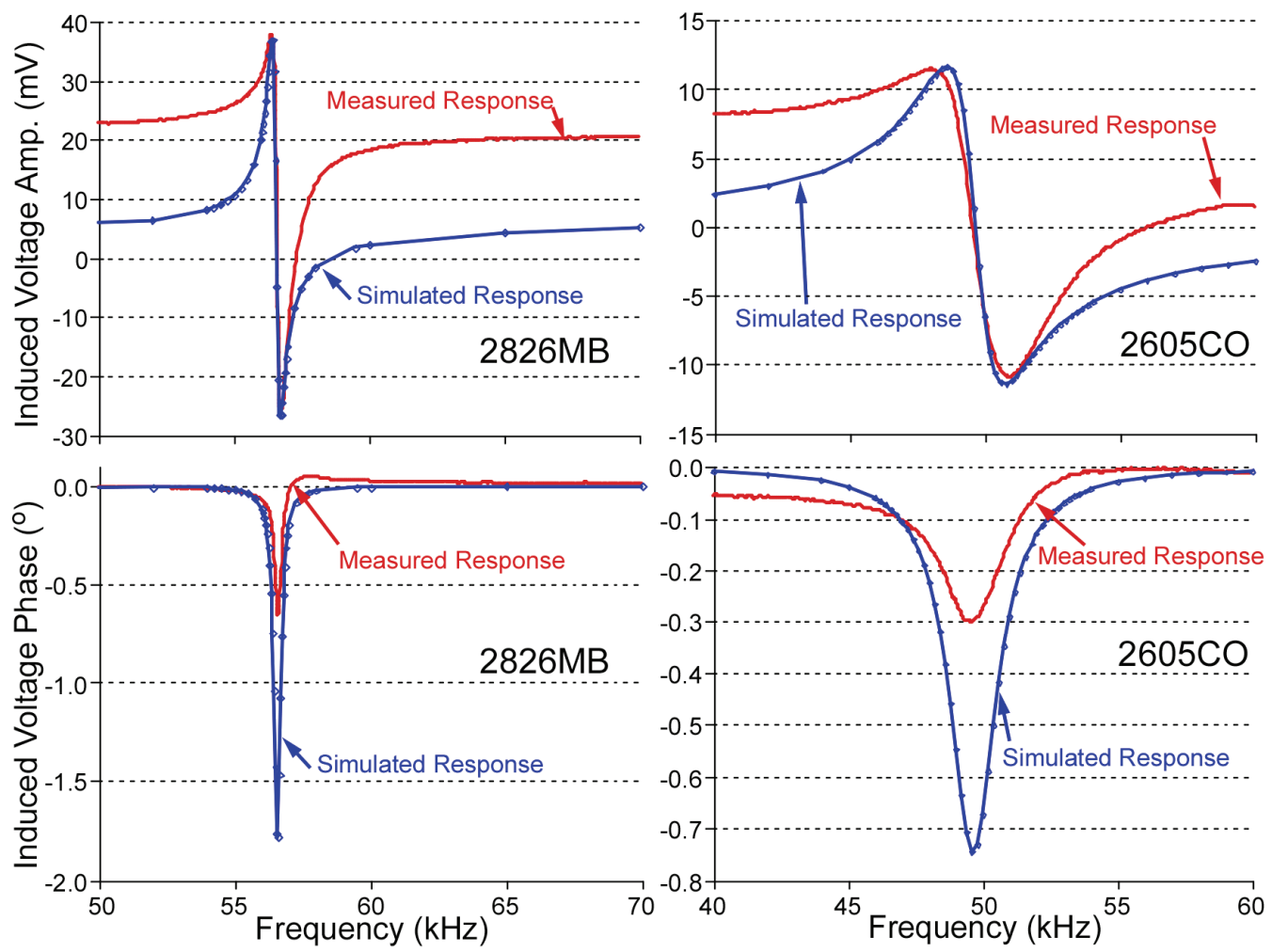

Fig. 2-11: FEA calculated and experimentally measured voltage amplitude (top) and

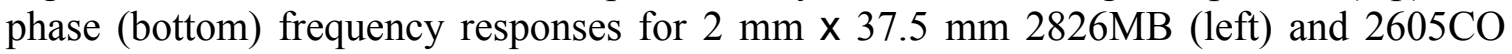
(right) sensors. The experimental response is fitted by the calculated response with slight modification of material parameters given in literature.

The same proportionalities between signal amplitude, the square of magnetostrictivity, and the inverse of the fifth-root of permeability found with the 
analytical model are also found with the FEA model. Note that displacements calculated with the analytical model for the ribbon sensor match those predicted by the FEA within $\sim 10 \%$, while resonant and anti-resonant frequencies generally agree within $\sim 5 \%$. Less agreement between the calculated signal amplitudes was found, usually within $10-100 \%$. The main source of discrepancy between the two models is a coupled shape function $\varphi$ for the fully coupled FEA that is much different near resonance than the shape function calculated with a magnetostatic analysis and used in the analytical model. In other words, the coupling between the strain and the magnetic field affects the driving field near resonance. To capture this effect in future analytical models, it may be necessary to iterate near resonance or to describe the drive signal in terms of magnetic potential as imposed by the current in the transmit coil (as is done with the coupled FEA).

The FEA model is also used to analyze the complex designs that are discussed in Chapters 4 and 5 of this work, with specific results presented in the context of those chapters. 

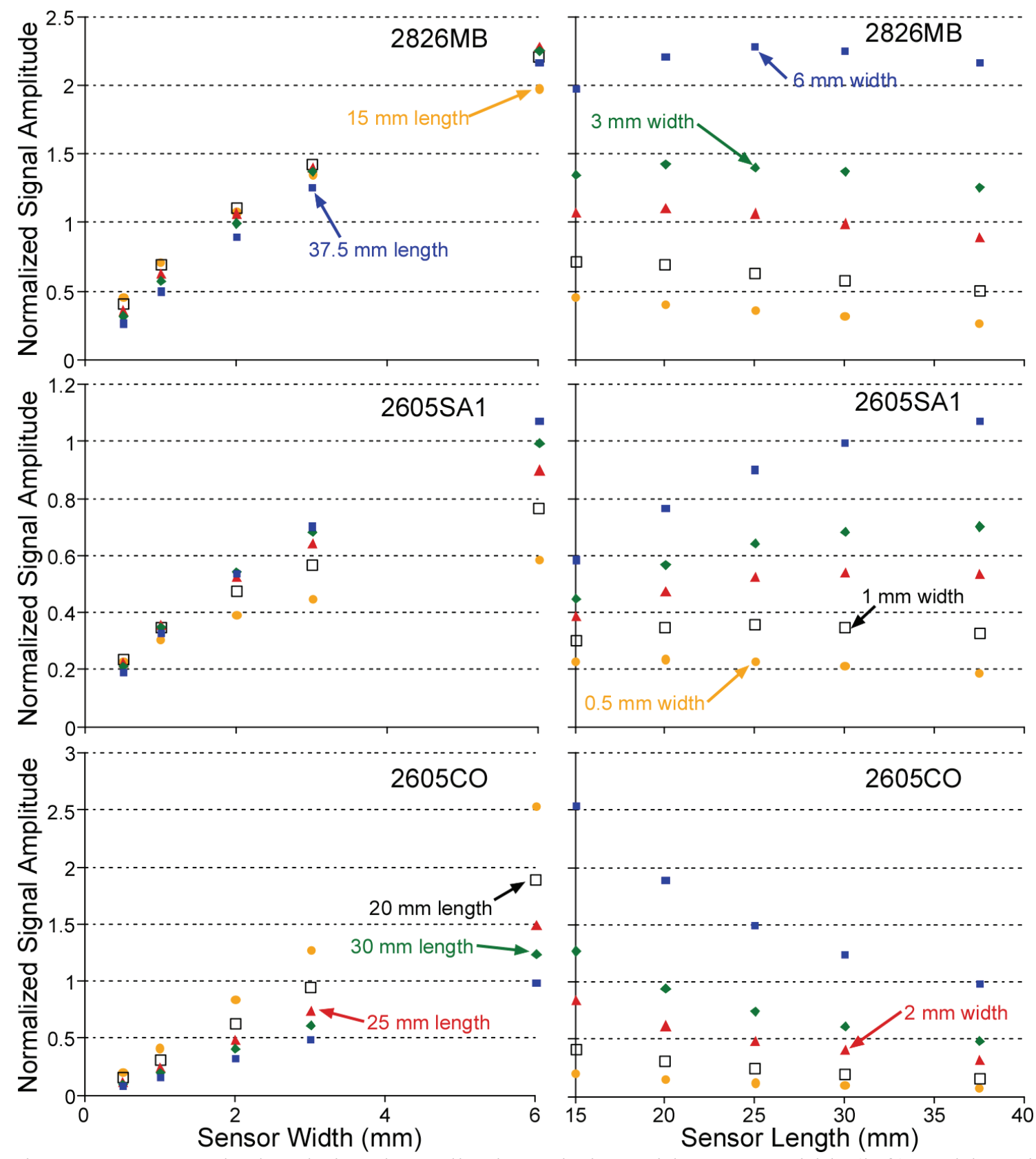

Fig. 2-12: FEA calculated signal amplitude variation with sensor width (left) and length (right) for three different materials: 2826MB (top), 2605SA1 (middle) and 2605CO

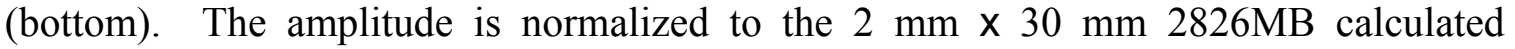
amplitude. Predicted trends generally match those predicted by the analytical model.

\subsubsection{Signal Stability in an Implanted Environment}

An important aspect of the sensor performance is the stability of the signal after long exposure to the corrosive environment of the bile duct. The indwelling time for stents 
ranges from less than 3 months to more than a year - assuming patency is maintained for that duration. As such, the sensor should maintain a stable signal - with changes related only to sludge accumulation or viscosity changes - in implanted conditions for at least a year. Additionally, the response of the sensor to a given mass load or viscosity should not change after long exposure periods.
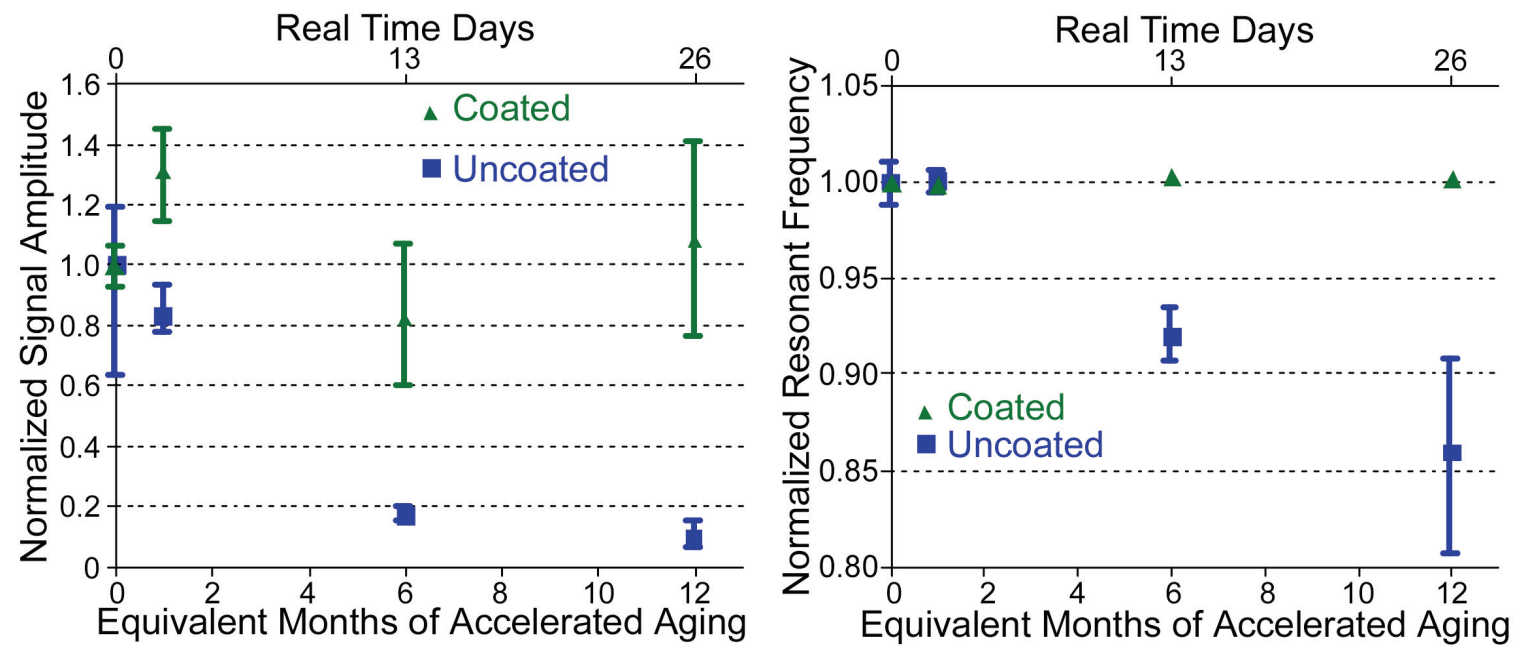

Fig. 2-13: Effects of long indwelling in bile were simulated by bathing sensors in saline at an elevated temperature $\left(60-65^{\circ} \mathrm{C}\right)$. Data points indicate the average of three sensors, while error bars indicate the range of measurements from the three sensors. Signal amplitudes and resonant frequencies were measured using extracorporeal configuration (B) (described in section 2.4). The sensor coated by parylene exhibited a much more stable response.

Generally, magnetoelastic materials contain iron, so corrosion is certainly a potential issue. Testing the final sensor design for over a year (real time) in simulated implanted conditions is often difficult due to time constraints and expense. However, accelerated testing can be performed in which the sensor is placed in a saline (or another bile simulant) bath at an elevated temperature to accelerate the corrosive effects. For instance, the Arrhenius equation predicts that, if the temperature is elevated to $60^{\circ} \mathrm{C}$, processes that 
occur after a year at $25^{\circ} \mathrm{C}$ will occur in 26 days [Hem98]. This theory is used to evaluate shelf-life of medical device packaging without waiting for real-time data, and can be used in this work to estimate the longevity of the sensor materials. In fact, accelerated testing (Fig. 2-13) has shown that the signal amplitude of $6 \mathrm{~mm}$ by $12.5 \mathrm{~mm} 2826 \mathrm{MB}$ ribbon sensors decreased by $90.7 \%$ while the resonant frequency decreased by $14 \%$ after an equivalent of 12 months immersion in saline. In comparison, sensors coated with $8 \mu \mathrm{m}$ of parylene and subjected to the same testing showed an average resonant frequency increase of $0.2 \%$ and an average signal amplitude increase of $8 \%$ - both similar to the repeatability of the sensors. Fig. 2-14 shows evident corrosion on exposed sensor surfaces. The act of coating the sensor with parylene reduced the signal amplitude by $35 \%$, but thinner coatings may provide sufficient passivation with a smaller effect on the signal amplitude (as briefly discussed in Chapter 5). It is important to point out that the results of the accelerated testing only illustrate some of the potential failure mechanisms that can be associated with long-term usage of the device. The accelerated testing relies on the assumption that failure processes can be predicted by the Arrhenius equation and associated kinetic coefficients; however, all failure mechanisms may not be governed by this equation. The results of real-time testing in end-use conditions are preferable when such testing is feasible, and such testing should be a part of future work for this application. 


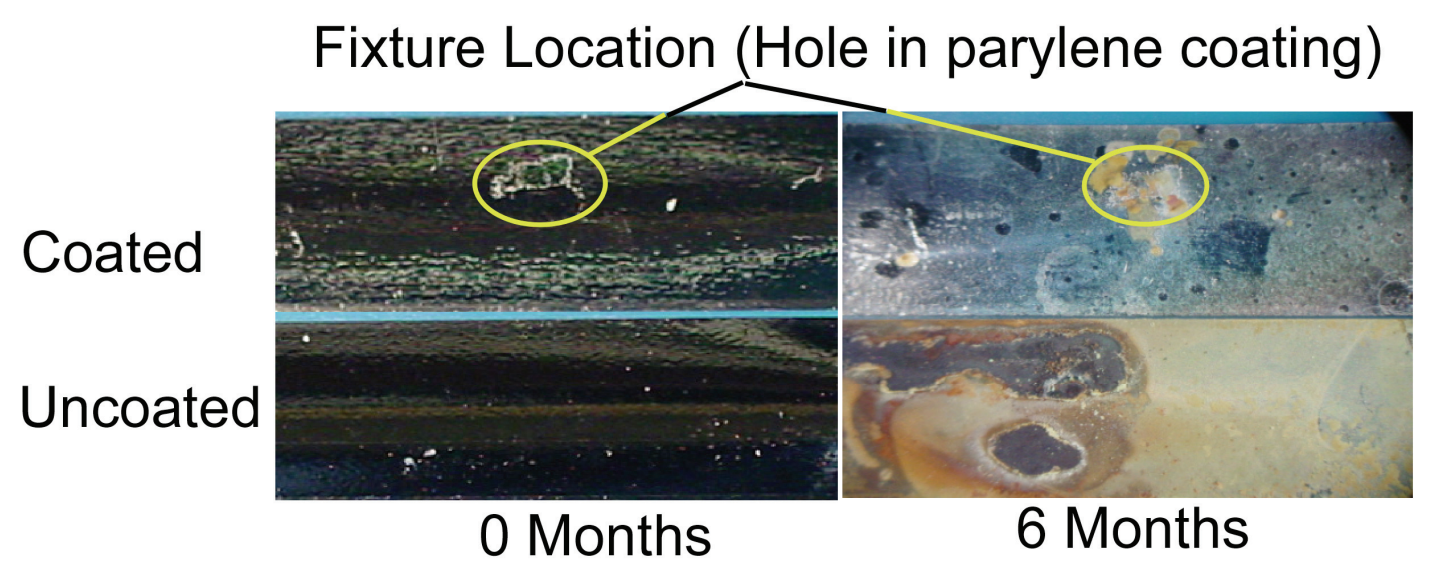

Fig. 2-14: Visual evidence of corrosion is present after 6 equivalent months (13 days) of accelerated aging wherever the sensor is not protected by parylene.

\subsubsection{Sensor Accommodation of Bending}

Because the bile duct is not generally a straight tube, the stent will need to accommodate some curvature along its length after deployment. The sensor is intended to be positioned near the sidewall of the stent, so the curvature experienced by the stent will also be experienced by the sensor. Because the sensor material is magnetoelastic with stress and magnetic domains strongly coupled - any stress induced by the curvature may significantly alter the signal that is available from the sensor, especially relative to a sensor under unstressed conditions. The specific amount of curvature that must be accommodated should be based on typical bile duct curvatures. Unfortunately, there is a dearth of quantitative data for bile duct curvature in the literature. Some guidance can be obtained from the design of "straight" plastic stents, which are actually manufactured with a radius of curvature of $\sim 70 \mathrm{~mm}$ to better fit the anatomy of the bile duct [Cla02, Sch90]. 


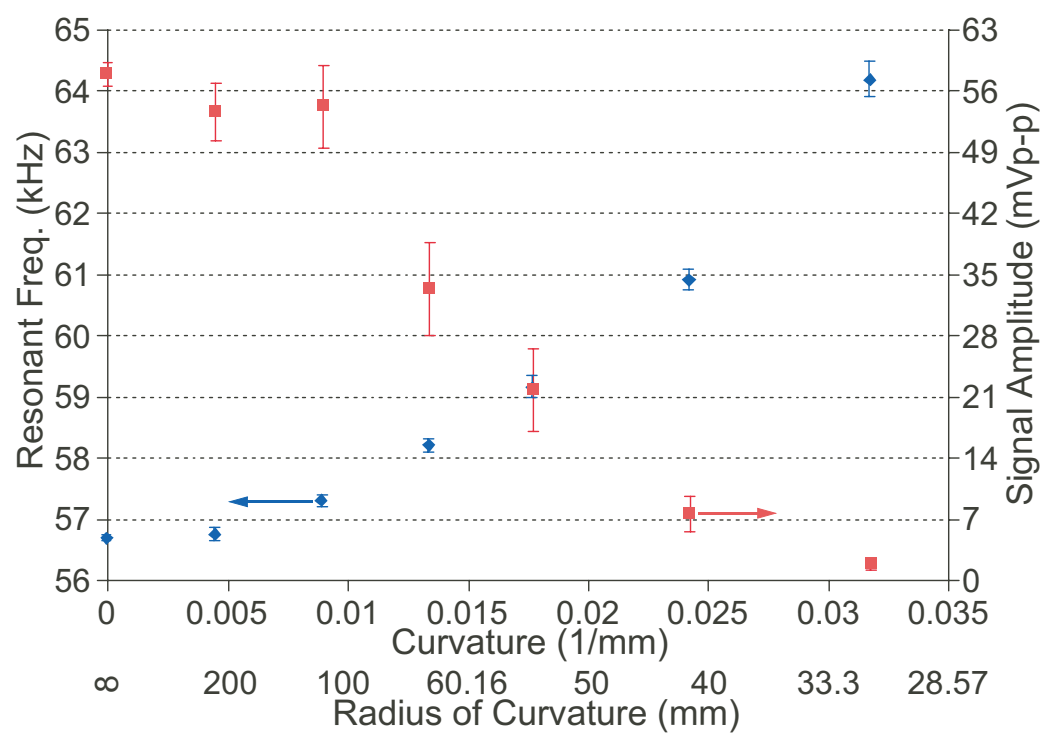

Fig. 2-15: Experimentally measured effect of forcing longitudinal curvature on an

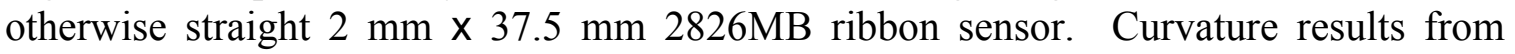
contact with the stent when inside a curved duct. Results are similar for ribbon sensors fashioned from other materials.

The performance of the sensor with regards to its accommodation of longitudinal curvature can be tested by integrating the sensor with the stent and placing in a flexible tube. The curvature of the tube can be varied and the resulting sensor amplitude and frequency response measured. To illustrate the potential performance of a simple sensor, a $2 \mathrm{~mm} \times 37.5 \mathrm{~mm} 2826 \mathrm{MB}$ ribbon sensor was tested with this method with results shown in Fig. 2-15. As can be seen, the frequency and amplitude response of the ribbon sensor changes significantly as the radius of curvature becomes smaller than about 110 mm. A slight change in amplitude and resonant frequency is allowable, as long as the normalized resonant frequency response to mass and viscosity is unchanged. 


\subsubsection{Sensor Design Summary}

The preceding sections illustrate the magnetoelastic operation of the sensor, which ties into the primary functional concern for the sensor: full-scale range. The full-scale range of the sensor is a combined measure of the robustness of the sensitivity and signal amplitude of the sensor as loads accumulate (i.e., as the stent fills with occlusions). Both sensitivity and signal amplitude should remain high as loads accumulate. For this application, the sensor should remain sensitive enough to discern between a patency (open flow channel) of $2 \mathrm{~mm}$ diameter and a completely occluded stent, all while providing a sufficiently measurable signal amplitude (defined for purposes of this work to be a signal-to-noise ratio of 4 at a wireless range of $7.5 \mathrm{~cm}$ ). Assuming the density of the sludge is near that of water, the mass of the sludge pertaining to a $2 \mathrm{~mm}$ diameter patency is approximately $4.5 \mathrm{x}$ the mass of the sensor for a $4 \mathrm{~mm}$ diameter stent up to $18 \mathrm{x}$ the mass of the sensor for a $10 \mathrm{~mm}$ diameter stent.

The magnetoelastic operation of the sensor is a primary functional concern. However, this application also presents other important issues that must be considered. First and foremost among these issues is the allowable sensor size. The size is constrained by the size of the stent - both in its expanded deployed state and in its compressed deliverable state. As such, the sensor must either be small enough to be unaffected by the compression, or it must elastically accommodate the compression. As mentioned previously, the stability of the sensor signal in the corrosive implanted environment is another concern, along with how well the sensor accommodates bending. Another issue is the attachment of the sensor to the stent. This attachment should be mechanically robust so the sensor does not break away from the stent. Additionally, the attachment 
should not hinder the resonant response of the sensor (e.g. through excessive anchor losses). These and other pertinent considerations are addressed where necessary in the performance goals of section 2.5 and in the discussion of specific designs in Chapters 3 through 6 .

\subsection{Magnetic Bias Design}

Our preliminary reports on magnetoelastic wireless sensing of sludge accumulation utilized externally applied AC interrogative and DC biasing magnetic fields [Ric07]. As mentioned in section 2.2.1, the resonant frequency of the magnetoelastic sensor is dependent on the orientation and strength of the applied DC biasing field due to the $\Delta \mathrm{E}$ effect (Fig. 2-16). Because the orientation of the interrogation equipment with respect to the sensor can vary on a test-to-test or patient-to-patient basis, a DC field emanating from this equipment may falsely shift the resonant frequency and result in an erroneous diagnosis. It is therefore desirable to integrate permanent magnets with the stent. This results in a fixed orientation between the field and the sensor, as well as a fixed field strength, reducing a possible source of measurement error.

A brief analysis into the expected ramifications of the $\Delta \mathrm{E}$ effect begins with relating the Young's modulus of the sensor to the applied DC magnetic field:

$$
E_{\text {app }}=E_{o}+g\left(H_{D C}\right) \text {, }
$$

where $E_{a p p}$ is the apparent Young's modulus of the sensor, $E_{o}$ is the Young's modulus with zero bias, $H_{D C}$ is the applied DC magnetic field strength, and $g$ is a generally nonlinear function relating the bias field strength to the incremental modulus change. For 
the purposes of this work, $H_{D C}$ varies over a relatively small range (0-5 Oe), and over this range $g$ can be represented as a proportional factor of around 21-31 $\mathrm{GPa} / \mathrm{Oe}$ for Metglas $^{\mathrm{TM}} 2826 \mathrm{MB}$ [And82]. The zero-bias Young's modulus for this alloy is 100-110 GPa. With these values, we see that a change in bias field strength from 3 Oe to $2.7 \mathrm{Oe}$ (a 10\% decrease) results in a change in apparent Young's modulus from nominally 183 GPa to nominally $175.2 \mathrm{GPa}$ (a $4.3 \%$ decrease). Finally, since the resonant frequency of the sensor is roughly proportional to the square root of the Young's modulus, a 2\% shift in resonant frequency results. A shift of this size is quite significant relative to shifts expected for mass and viscosity loads in this application. This analysis highlights the need to maintain a consistent bias field to reduce unwanted variability in the measurand.
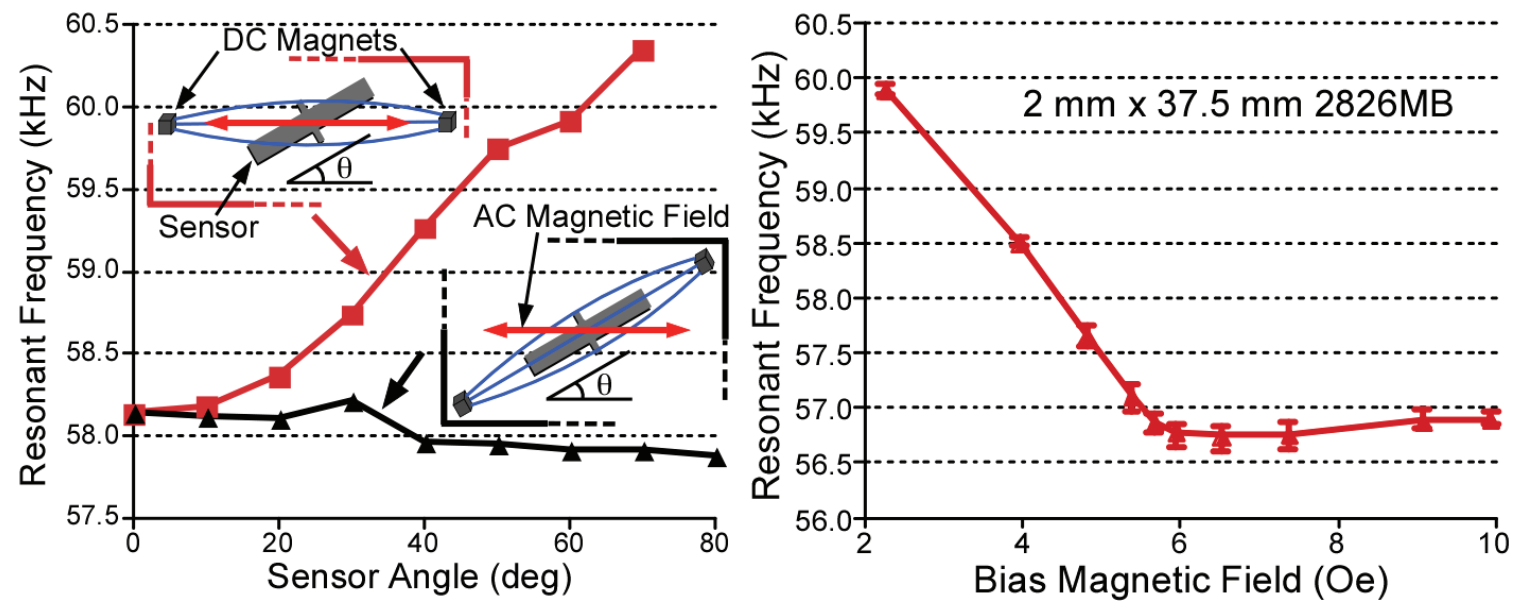

Fig. 2-16: LEFT: The red trace shows a large frequency shift of up to $2 \mathrm{kHz}$ occurs when the relative DC field orientation varies. However, maintaining the orientation of the DC field to the sensor renders the resonant frequency insensitive to the AC field strength and orientation. RIGHT: Similarly, shifting the strength of the applied field can result in a $3.25 \mathrm{kHz}$ shift. Both measurements are from $2 \mathrm{~mm}$ x $37.5 \mathrm{~mm} 2826 \mathrm{MB}$ ribbon sensor.

In order to integrate magnets with the stent, biocompatibility of potential designs must be reasonably assured. The majority of the reports on the use of permanent magnets 
in biomedical implants are focused on intra-oral magnets for dentistry and orthodontic purposes. Though the intra-oral environment is not identical to that of the bile duct, these investigations do allow some conclusions to be drawn regarding the biocompatibility of permanent magnets. The first factor regarding the biocompatibility of implanted magnets is the effect on nearby tissues of chronic field exposure. Research shows that the response of buccal mucosa (the lining of the cheeks and lips) exposed to a magnetized implant with 80-140 Oe field strength is negligibly different from the response of the same tissue exposed to a demagnetized implant [Bon98]. Again, it is expected that the application described here requires a field strength of less than $10 \mathrm{Oe}$, so these findings suggest that chronic magnetic fields of the size and strength required do not pose a significant biocompatibility risk. The second factor regarding the biocompatibility of permanent magnets is the cytotoxic nature of the constituent material of the magnet or any potential corrosive products. This factor can be accounted for either by appropriate choices of inert permanent magnetic materials, or by coating the permanent magnet with a layer of passivating, biocompatible material. For instance, literature notes that although bare neodymium magnets are susceptible to corrosion in saliva - especially in the presence of bacteria - a parylene coating is effective in protecting against corrosion [Wi195].

In order to maintain the flexibility and minimally invasive nature of self-expanding biliary stents, it is important to optimize the distribution of small magnets and still provide a fairly uniform magnetic field. Common magnetostatic finite element tools such as those offered in Ansoft, COMSOL, and ANSYS can be used for these purposes provided an estimate of remanent magnetization for the magnetic material can be made. 
The use of these tools as they relate to this work is described in the specific system design sections in Chapters 3, 4, and 6.

\subsection{Interrogation Module Design}

The interrogation module refers to all of the external (i.e. not implanted) components that are required to send and receive alternating magnetic field information to and from the sensor. There are four main components of the interrogation module as it currently stands: a network analyzer, an amplifier, transmit coil(s), and receive coil(s). These components are discussed in more detail in the following sections.

\subsubsection{Magnetic Field and Flux Generation and Measurement}

The interrogation module is driven and measured by a HP 4395A Network Analyzer. Although the final version of the wireless stent monitoring system could make use of circuitry and/or software that is specifically designed for measuring frequency response characteristics and providing medical diagnostic assistance, the network analyzer is a more general instrument that allows significant flexibility and streamlined signal processing, as is appropriate for research. For instance, the network analyzer allows measurement of amplitude, phase, and delay (among others) while also allowing manual adjustment of measurement bandwidth and sweep averaging. The analyzer also can be programmed to subtract common mode signal from the displayed trace. All of these functions can be used to optimize the signal-to-noise ratio and facilitate investigation of system performance. For all results presented in this work, a $1 \mathrm{kHz}$ integration 
bandwidth is used (resulting in a sweep time of $460 \mathrm{~ms}$ ), and 32 sweeps are averaged together to minimize noise. Additionally, the common mode coupling between the transmit and receive coils (described in section 2.4.3) is measured without the sensor in place, and the network analyzer is programmed to subtract this baseline signal from the measurement taken with the sensor in place.

The output signal from the $4395 \mathrm{~A}$ is amplified prior to reaching the transmit coils. A commercial power amplifier - such as the Krohn-Hite model 7500, as is used in this work (Chapter 6) - offers sufficient performance for the frequency and current requirements of this application. Alternatively, a custom amplifier circuit can be built. One such configuration used in this work (Chapters 3, 4, and 5) consists of two common emitter stages for voltage amplification followed by two emitter-follower stages for current amplification. The input and output are capacitively coupled to the spectrum analyzer and transmit coil respectively. The circuit is loosely based on an audio amplifier design by Marshall Leach [Lea05], operated in open loop to maximize bandwidth at the expense of linearity and/or gain. One advantage of the commercial amplifier over the custom amplifier is better stability due to improved thermal management systems integrated in the commercial amplifier.

The inductive transmit coils could be driven at resonance by using a capacitor connected to them in parallel with the network analyzer and amplifier as in [Ong05] to increase the current and resulting magnetic field. By tuning the capacitor, the resonant frequency of the coils could match the resonant frequency of the sensor. However, tuning the capacitor over the large range in resonant frequency expected (on the order of $50 \%$ change in frequency, as opposed to the $<1 \%$ expected in [Ong05]) is impractical and 
thus not done in this work.

\subsubsection{Transmit and Receive Coils}

Important factors in the design of the transmit and receive coils include crosssectional area and length of the coils, number of turns, density of winding, wire gage size and type (solid versus stranded), core material, and relative orientation of the coils. Each

of these factors has a role in determining the equivalent inductance, capacitance, and resistance of the coil, along with the coupling between coils and to the sensor. For the transmit coil, a large number of turns is beneficial in developing a large magnetic field for a given current; however, a balance must be struck so that the number of turns does not increase the inductance and choke the current that can be delivered by the amplifier at the frequencies of interest. For the receive coil, a large number of turns can again be beneficial for developing a large induced voltage; however, care must be taken to not add so much inductance and capacitance (by densely packing the turns) that the resonant frequency of the coil falls below the frequencies of interest. Another tradeoff lies in the cross-sectional area of the coil, as a large area increases the potential for generating a large voltage for a given amount of flux density but also increases the opportunity for picking up stray flux not developed by the sensor (and thus adding to the noise of the system). Likewise, introducing a high permeability core in the receive coil improves the potential for gathering flux from the sensor; however, this also means that cross-coupling to the transmitted signal or ambient flux may increase the measured noise level. For the purposes of this work, three different wireless coil configurations were used to evaluate performance of the systems, as shown in Figure 2-17. 

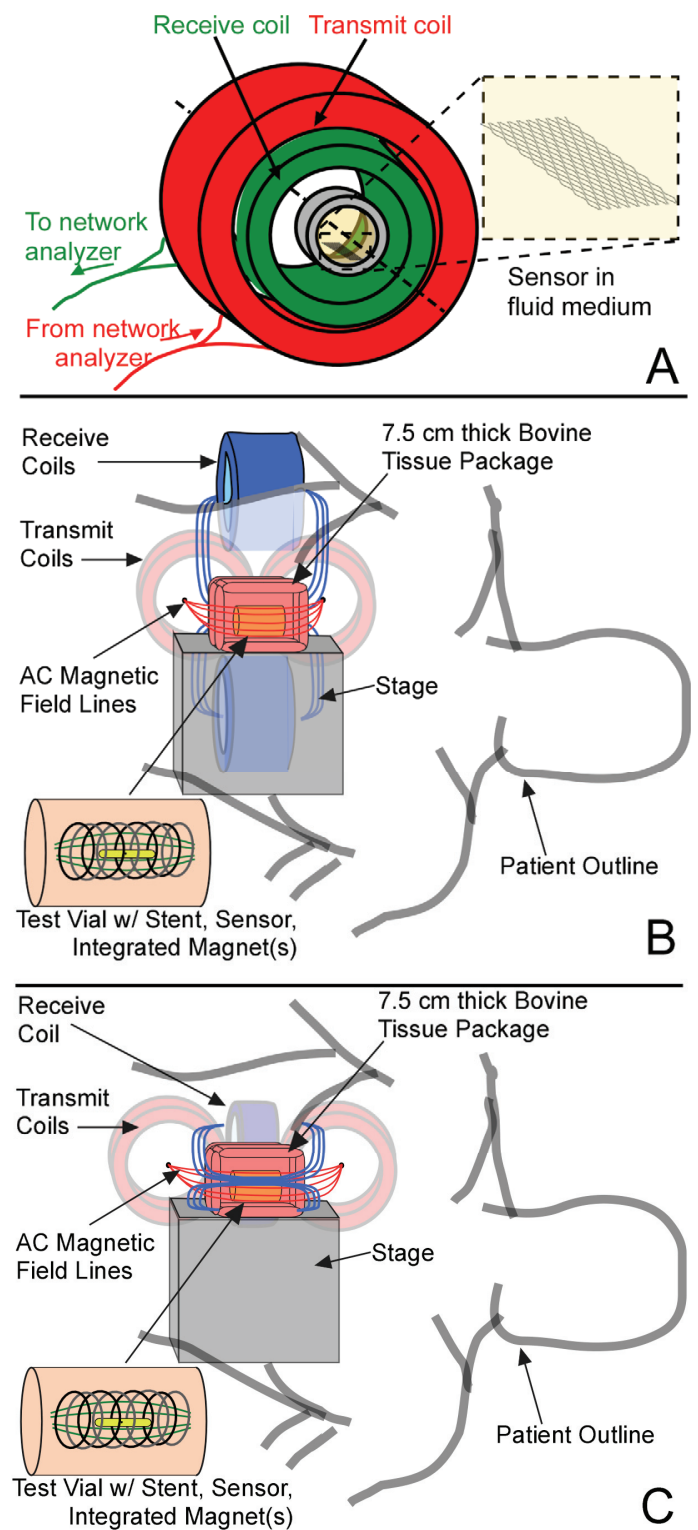

Fig. 2-17: Coil configurations used in this work. Throughout this work, they are referred to by the letter designation in this figure. A) Coaxial configuration. Dual Helmholtz coils (not shown) on either end of the transmit and receive coils can be used to provide a uniform and well-controlled bias field to isolated sensors.

B) Extracorporeal configuration. C) Alternative extracorporeal configuration. In each configuration, a vial filled with viscous media simulates bile, and in the extracorporeal setups, a $7.5 \mathrm{~cm}$ thick package of bovine tissue can be used to simulate the potential signal attenuation due to the intervening tissue of the patient. Differences between the test setups in measured signal amplitude were consistent regardless of sensor geometry, sensor material, or sensor environment.

The extracorporeal configuration (B) is intended for use in the final application, after 
the stent and sensor are implanted. In this setup, coils are configured such that the transmit coils and receive coils can both couple to the sensor, but not with each other. The interrogative field lines emanate from one transmit coil, loop through the sensor position such that the field lines are longitudinally aligned with the sensor, and terminate in the other transmit coil. The response field lines emanate from the sensor and are in essentially the same pattern as they would be if emanating from a dipole magnet. Thus, the receive coils are located at null points of the transmitted signal (i.e. are oriented perpendicular to the direction of the transmit field), yet are aligned with the resulting field from the sensor. This arrangement helps to decouple the transmit signal from the received signal, improving the signal-to-noise ratio. It is intended that the coils be placed on the back of the patient as shown in Fig. 2-1. This position was chosen because there tends to be less variation in intervening tissue thickness from person to person in this region as opposed to the anterior abdomen.

Because the exact location of the interrogation equipment with respect to the sensor can vary on a test-to-test or patient-to-patient basis, it is important to characterize the robustness of the signal as this relative location is varied. Figure 2-18 shows the variation in signal amplitude as the position of a $6 \mathrm{~mm} \times 37.5 \mathrm{~mm}$ ribbon sensor is varied with respect to the extracorporeal interrogation equipment. A symmetric decrease in the signal amplitude is found when the sensor is moved in the cranial-caudal direction, with at least $80 \%$ of the signal still available with an offset of up to $+/-2 \mathrm{~cm}$. Movement of the sensor in the medial-lateral direction results in a wider yet somewhat less symmetric signal magnitude distribution; at least $80 \%$ of the signal is still available with an offset of up to $+/-5 \mathrm{~cm}$. The lack of symmetry in this distribution is not significant compared to 
repeatability of the signal amplitude. Finally, movement in the posterior-anterior direction - which equates to movement closer to or farther from the coils - approximately shows the expected inverse cubic relationship between signal strength and distance. At least $80 \%$ of the signal is still available if the sensor is located $0.5 \mathrm{~cm}$ deeper into the tissue (in addition to the standard test setup of $7.5 \mathrm{~cm}$ ). The measured resonant frequency shifts only by approximately $+/-0.3 \%$ over this entire volume, which is similar to the testto-test repeatability of a consistently positioned sensor.
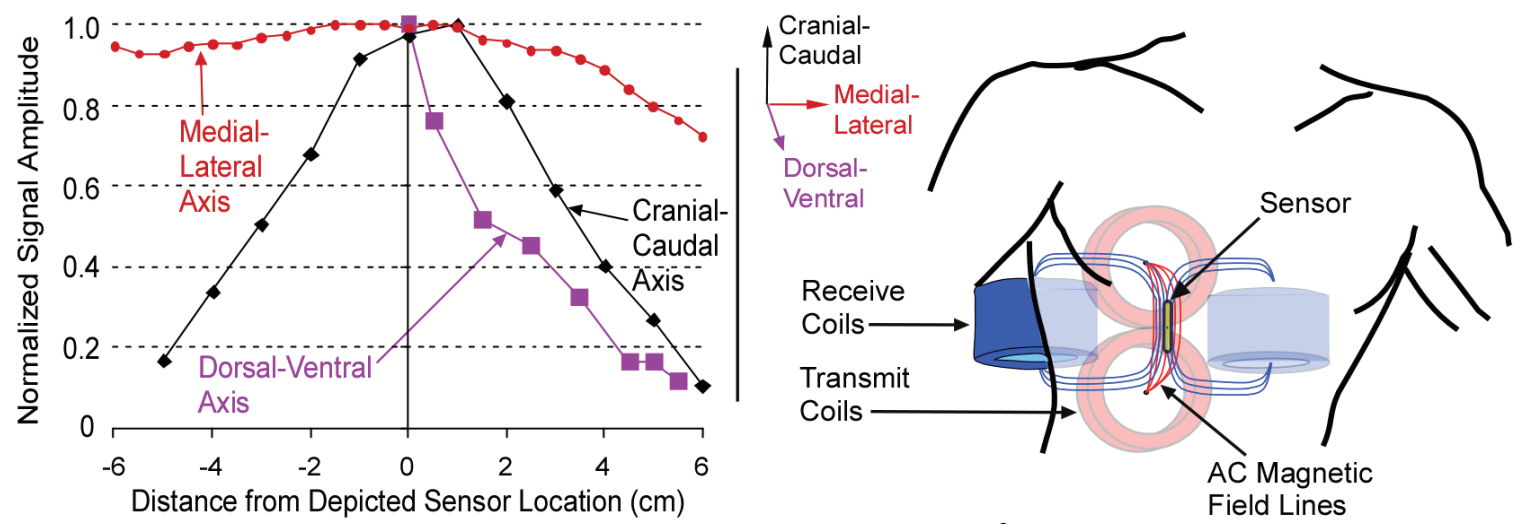

Fig. 2-18: Normalized signal amplitude for a $37.5 \times 6 \mathrm{~mm}^{2}$ sensor at various locations to characterize the extracorporeal configuration (B) performance. The cranial-caudal axis range could be increased by separating transmit coils while boosting signal amplitude and using shorter sensors.

An alternative extracorporeal configuration (C) is similar to configuration (B) and uses the same general concept for coupling to the sensor with both transmit and receive coils but limiting the coupling between the coils. However, configuration (C) spreads the transmit coils out and locates the (single) receive coil directly in between the two. The receive coil is also no longer cylindrical, but rather is elongated in one direction and flattened in the perpendicular direction (see Fig. 2-19). These changes allow more of the cross-sectional area of the receive coil to be closer to the sensor while still existing in a 
null point of the transmitted signal, boosting the signal-to-noise-ratio of the system by a factor of 2. Additionally, the number of turns per meter in the dual-layered transmit coils have been chosen to maximize the signal-to-noise ratio for this setup.

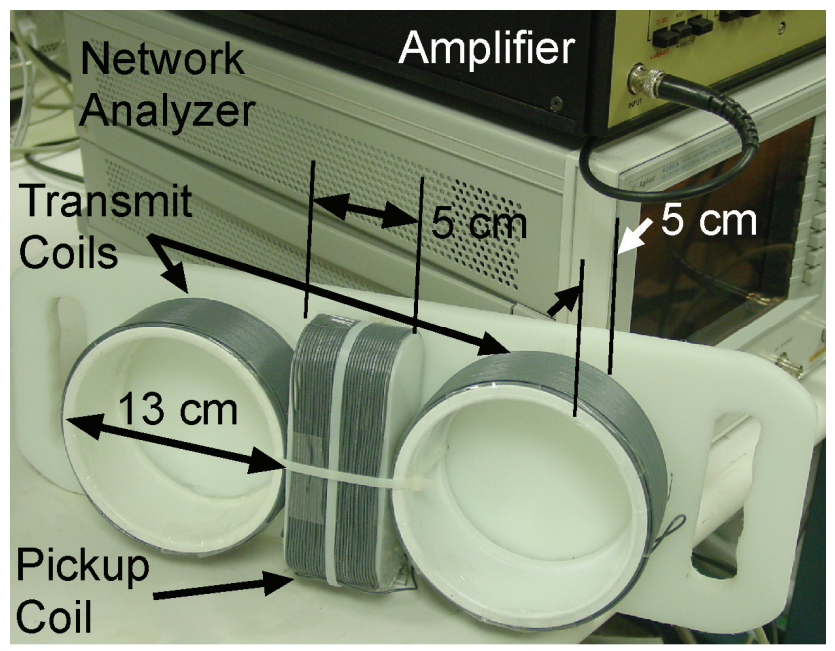

Fig. 2-19: Extracorporeal configuration (C) as fabricated, with important dimensions.

The spatial performance of configuration (C) is characterized in Figure 2-20. In Figure 2-20A, the $2 \mathrm{~mm} \times 37.5 \mathrm{~mm}$ sensor is aligned parallel with the $\mathrm{X}$ axis (cranialcaudal). In the $\mathrm{Z}$ direction (dorsal-ventral), the signal scales with $1 / \mathrm{z}^{3}$. In the $\mathrm{X}$ (cranialcaudal) and $\mathrm{Y}$ (medial-lateral) directions, the signal is symmetrically distributed. Spreading the transmit coils out results in a small improvement in the available signal as the sensor is offset in the $\mathrm{X}$ direction. In Fig. 2-20B, the sensor has been rotated in the X$\mathrm{Z}$ plane by 45 degrees as shown in the inset schematic. This rotation results in a larger signal being available when the sensor is offset in the negative $\mathrm{X}$ direction such that the sensor is aligned with the field lines coming out of the transmit coils. However, an offset in the positive $\mathrm{X}$ direction results in a much smaller available signal because the field lines emanating from the transmit coils are now mostly transverse to the sensor. For a 
sensor that is parallel with the $\mathrm{X}$ axis, at least $80 \%$ of the signal available at $(0,0,7.5 \mathrm{~cm})$ is available with an $\mathrm{X}$ offset of $+/-3 \mathrm{~cm}$, or with a $\mathrm{Y}$ offset of $+/-5 \mathrm{~cm}$. Again, the measured resonant frequency varies only by approximately $+/-0.3 \%$ over the entire measured volume.

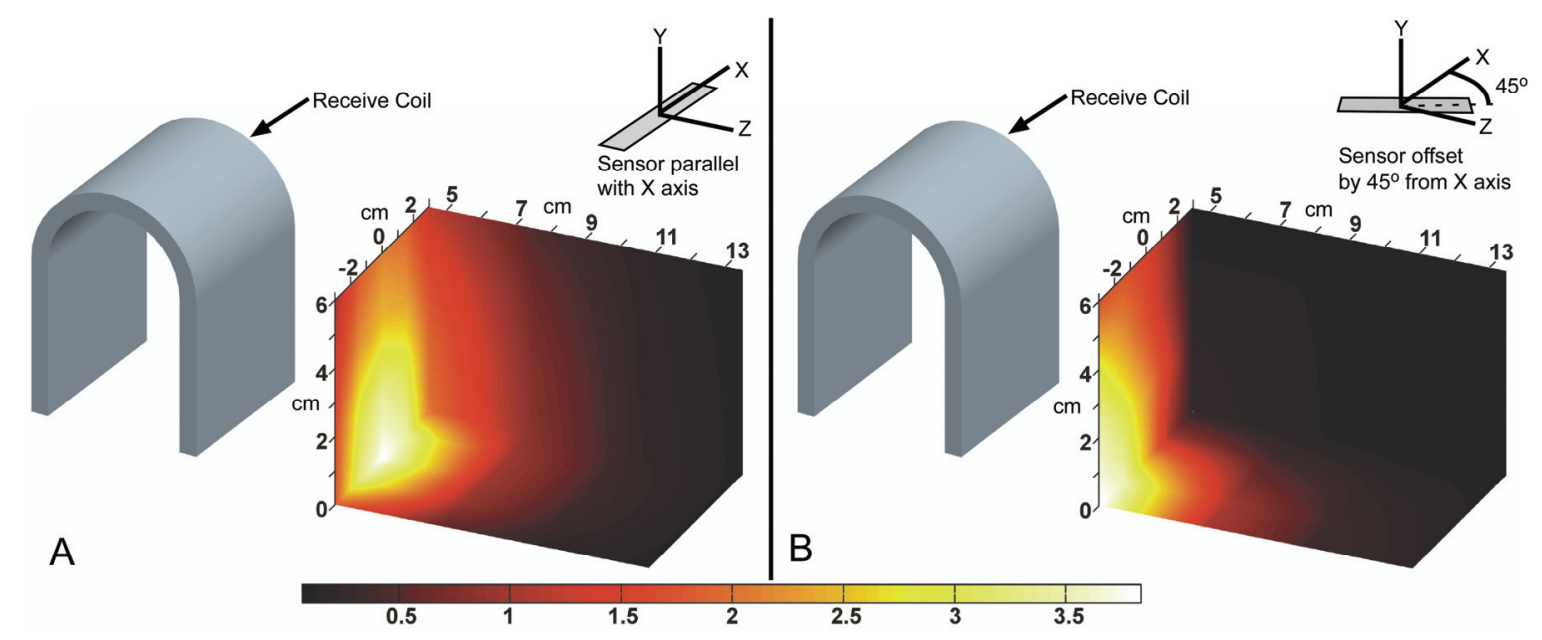

Fig. 2-20: Spatial characterization of extracorporeal configuration (C). A) Sensor aligned parallel with faces of coils. B) Sensor rotated $45^{\circ}$ in the X-Z plane. The spatial characteristics of both sensor alignments are symmetrical about the $X-Z$ plane. The signal amplitude is normalized to that available from the sensor at the coordinates $(0,0$, $7.5 \mathrm{~cm}$ ) and parallel with the $\mathrm{X}$ axis.

The extracorporeal configurations described above spatially decouple the transmitted signal from the received signal in order to improve the signal-to-noise ratio. An alternative approach to decouple the signals is to separate the two temporally. This approach is described in [Ong05]. The transmit and receive coils are coaxial and concentrically wrapped around the patient. The receive circuitry is shut down while an excitation signal at the expected resonant frequency is sent through the transmit coil to build up a response in the resonant sensor. Then, the excitation signal is stopped and the resonant sensor rings down at its damped natural frequency while the receive coils 
measure. This approach requires a significant ring-down period so the response can be adequately measured. For the application described in this work, in which large heavilydamped loads are expected to be applied to the sensor, it is unclear whether the resulting ring-down response would be measurable. For this reason, this work uses the spatially decoupled approach described previously.

Table 2-3: Coil configuration comparison

\begin{tabular}{|c|c|c|c|}
\hline & Coaxial $(\mathrm{A})$ & Extracorporeal (B) & Extracorporeal (C) \\
\hline Transmit Coil Diameter $(\mathrm{cm})$ & 15.7 & \begin{tabular}{|l|}
13 \\
\end{tabular} & 13 \\
\hline Transmit Coil Turns/Length $(1 / \mathrm{m})$ & 588 & 1275 & 1120 \\
\hline Transmit Coil Length $(\mathrm{cm})$ & 8 & 8.5 & 5 \\
\hline Receive Coil Dimensions (cm) & 10.3 (dia.) & 13 (dia.) & 5 wide $\times 13$ tall \\
\hline Receive Coil Turns/Length $(1 / \mathrm{m})$ & 614 & 514 & 620 \\
\hline Receive Coil Length $(\mathrm{cm})$ & 4.4 & 7 & 5 \\
\hline $\begin{array}{c}\text { Longitudinal AC Magnetic Field } \\
\text { Amplitude at Sensor @ } 60 \mathrm{kHz}(\mathrm{A} / \mathrm{m}) \\
\text { (7.5 cm from extracorporeal setup) }\end{array}$ & 20.5 & 12 & 6.5 \\
\hline $\begin{array}{c}\text { Current Amplitude in Transmit Coil @ } \\
60 \mathrm{kHz}(\mathrm{mA})\end{array}$ & 38 & 95 & 87 \\
\hline $\begin{array}{c}\text { SNR for Unloaded } 2 \mathrm{~mm} \times 37.5 \mathrm{~mm} \\
\text { 2826MB Ribbon Sensor }(7.5 \mathrm{~cm} \text { from } \\
\text { extracorporeal setup) }\end{array}$ & 210 & 25 & 50 \\
\hline
\end{tabular}

The coaxial configuration (A) is used for bench-top testing and mainly for initial evaluation purposes. In this setup, transmit and receive coils are concentrically oriented. The device under test is also placed in the center of this setup. With this setup, isolated sensors can be evaluated using a uniform and controllable bias field applied by Helmholtz coils located coaxially with the long axis of the sensor, outside the transmit and receive coils. The main advantages of this setup over the extracorporeal setup are that it easily achieves a higher signal-to-noise ratio, and recorded signal amplitudes are less dependent on precise sensor positioning. However, this setup cannot be used to 
replicate conditions when the sensor is fully implanted, as concentric coils of the size used would lie inside the patient - an obviously undesirable situation. The bile duct runs approximately vertically in an upright patient, so a coaxial wireless setup could be used if the transmit and receive coils were large enough to fully encircle the patient (as in [Ong05]). Important parameters for both of the extracorporeal and the coaxial coil configurations are listed in Table 2-3.

\subsubsection{Effects of Tissue, Metal Implanted Objects, and Nearby Structural Objects}

Although the design of the extracorporeal configurations is intended to place the receive coils at a null point of the transmitted signal, there is still a certain amount of coupling between the transmit and receive coils that results in an induced voltage on the receive coil. The amplitude and phase of the induced voltage is frequency dependent, and in general the frequency dependence is large enough to obscure the frequency response of the sensor. Fortunately, the "common-mode" baseline coupling between the transmit and receive coils can be measured while the sensor is not present, and then subtracted from the overall signal measured while the sensor is present. As long as the baseline coupling does not appreciably shift once the sensor is present, the resulting compensated measurement will exhibit only the frequency response of the sensor, as

desired. Of course, the presence of the sensor next to the interrogation coils can also bring a number of materials into the near-field area adjacent to the coils, including biological tissues, other metal implants, and possibly medical furniture like a surgical table or chair. As such, it is important to quantify the effects of such materials. 
First, a survey of some applicable electromagnetic field theory is warranted. At the frequencies used in this work (between $20 \mathrm{kHz}$ and $250 \mathrm{kHz}$ ), the free space wavelength of the magnetic field ranges between 1200 and $15000 \mathrm{~m}$. Thus, quasi-static field theory (i.e. the magnetic field and electric field of the transmitted signal are weakly coupled) is applicable because these wavelengths are much greater than the lengths of interest in the system. Also, the effects of the magnetic field can be characterized as near-field interactions, as evidenced by the $\sim 1 / \mathrm{r}^{3}$ dependency of the sensor signal shown in Figs. 218 and 2-20. Knowing that the electromagnetic fields at work in this system fall into these categories helps to narrow the possible dominant effects. For instance, near-fields are strongly affected by mutual coupling between the generated magnetic field and nearby conductors, while quasi-static magnetic fields typically generate only small eddy currents that lead to negligible heating.

The coupling between the coils and the nearby conductors (such as bodily tissues, implants, and parts of medical furniture) is governed by the permeability and permittivity of the conductors. Bodily tissues are generally nonmagnetic, with permeability very similar to that of air or free-space. However, bodily tissues have a relative permittivity with distinct frequency dependence - varying from $10^{3}$ at $20 \mathrm{kHz}$ to about $10^{2}$ at $250 \mathrm{kHz}$ for muscle tissue, for instance [Dur92]. Conductivity of muscle tissue also varies over the frequency range of interest, from $0.1 \mathrm{~S} / \mathrm{m}$ at $20 \mathrm{kHz}$ to $0.5 \mathrm{~S} / \mathrm{m}$ at $250 \mathrm{kHz}$. Other tissues have different conductivity and permittivity, but the general idea is similar. With metal and structural objects, both permeability and permittivity are different than air and generally exhibit a frequency dependent value. For example, the relative permeability of Metglas $^{\mathrm{TM}} 2826 \mathrm{MB}$ varies from 30000 at $200 \mathrm{~Hz}$ to about 1000 at $50 \mathrm{kHz}$ [Met08]. The 
fact that these materials are conductive results in eddy currents due to the time-varying magnetic field supplied by the coils. The presence of charge (due to eddy currents) in any conductor will affect the mutual coupling between the conductor and the coils, causing a change in current in the coils. The fact that the permeability, conductivity and/or permittivity of the conductor materials changes with frequency means the eddy currents will also change with frequency, shifting the mutual coupling (i.e. shifting the baseline) when these conductors are brought near the coils.

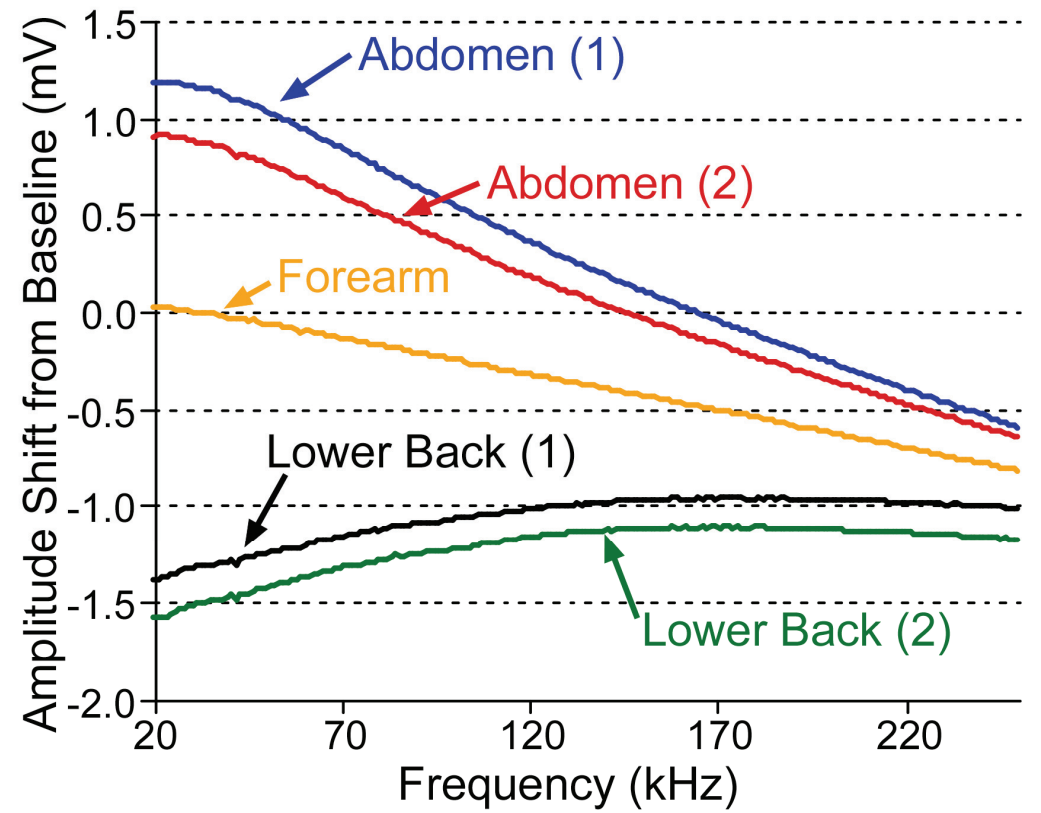

Fig. 2-21: Shifts in baseline amplitude due to presence of various portions of a human body.

The extracorporeal configuration (C) was used to quantify the baseline shifts that can be expected from the presence of biological tissues, small metal objects, and medical furniture. For biological tissues, the coils were locked into a stable position and a baseline was taken. Then, a portion of a human volunteer - including the lower abdomen, lower back, and forearm - was placed next to the coils and the baseline shift was 
measured, with data displayed in Fig. 2-21. Note that, for a given portion of the body, the frequency dependence of the baseline shift is similar (with a frequency independent shift between the readings for a given portion of the body). However, different body portions give different frequency dependencies, signifying that relative amounts of muscle, fat, bone, or other tissues may have an effect. The size of the baseline shifts due to the biological tissue is of the same order of magnitude as the sensor response.

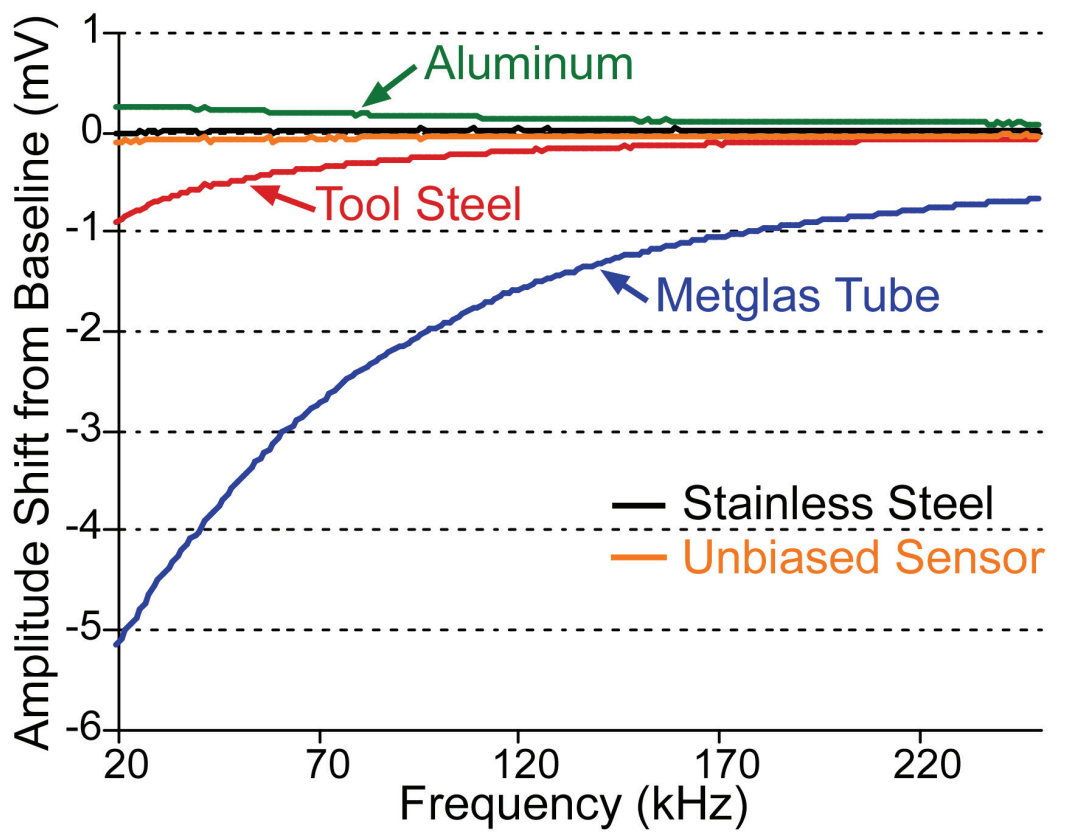

Fig. 2-22: Shifts in baseline amplitude due to presence of various small metal objects.

A patient may have other implants in addition to the biliary stent. To quantify the baseline shifts that may occur due to small metal objects similar to implants, the coils were again placed in a stable position, and a baseline was taken. Then, various metal objects were placed $7.5 \mathrm{~cm}$ away from the face of the pickup coil, with the long axis of the object parallel with the axis of the pickup coil. The baseline shift was measured, with results shown in Fig. 2-22. Note that, for the "non-magnetic" metals (including the 316 
stainless steel tube; some other stainless steels are magnetic), for small (sensor-sized) amounts of unbiased (and thus not actively resonating) Metglas ${ }^{\mathrm{TM}}$, and even for the standard steel parts, the shift is small relative to the expected sensor signal. However, for "large" amounts of the high permeability materials (like Metglas ${ }^{\mathrm{TM}}$ ) the shift is significant and cannot be ignored. Fortunately, most medical implants are not made of large amounts of high permeability metals, so baseline shifts due to the presence of implants are expected to be small.

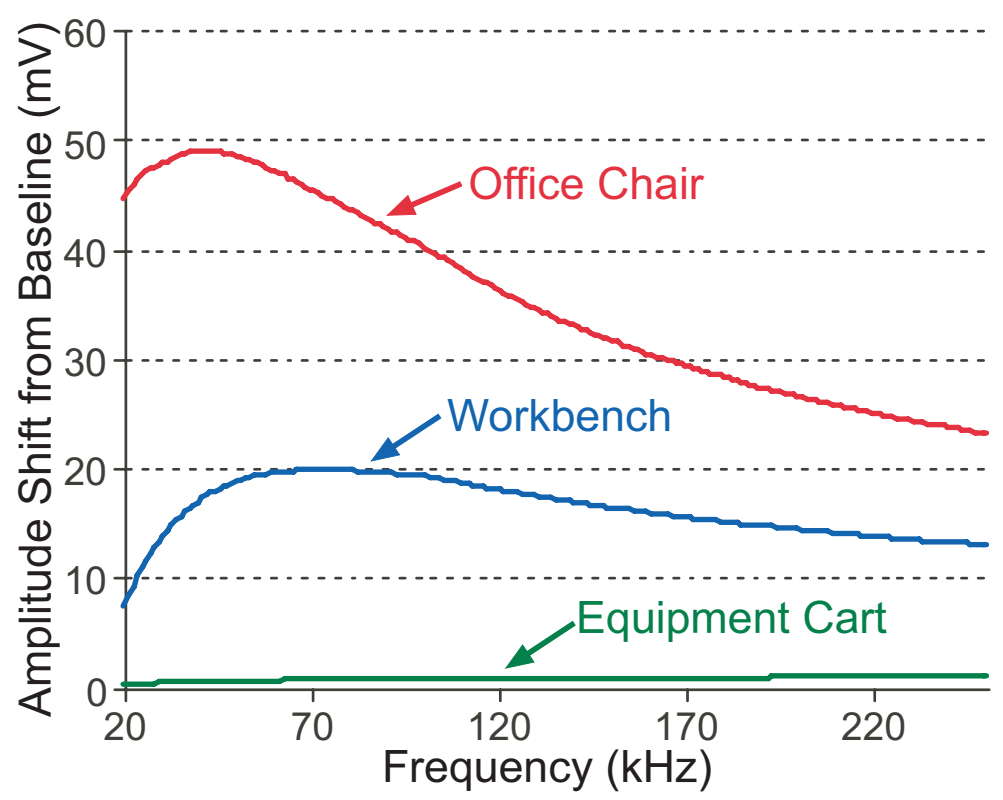

Fig. 2-23: Shifts in baseline amplitude due to presence of various pieces of lab furniture.

Another potential source of baseline shifts is the presence of medical furniture on which the patient may be laying or seated. To evaluate the baseline shifts that may occur due to such furniture, the coils were held by hand in a stable position, and a baseline was taken. Then, the coils were placed on a workbench, face down, and the baseline shift was measured. This was repeated with the seat of an office chair and the top of an equipment 
cart. The results are shown in Fig. 2-23. Note that in general the shifts due to these objects are quite significant with respect to the expected sensor signal. Thus, in the actual use of the system, it may be required that the environment around the patient and coils be well controlled - specifically, the patient may need to be brought to the coils after a baseline is taken, rather than the coils being taken to the location of the patient. For the experimental work described in Chapters 3, 4, and 5, the baseline was taken with the coils in a stable position, and then the sensor was placed in position without moving the coils. Further consideration of the effects of baseline shift, including methods for minimizing the shift, is given in Chapters 6 and 7.

It is important to also note other observations regarding the effect on the signal due to the presence of tissues and metal objects. First, note that the baseline signal (with the coils in air) varies monotonically from about $120 \mathrm{mV}$ at $20 \mathrm{kHz}$ to about $36 \mathrm{mV}$ at 250 $\mathrm{kHz}$. This frequency dependency is mainly due to the reduction of current in the transmit coils as the frequency increases (as a result of the inductance of the coils). Thus, only the structural objects result in more than a $10 \%$ absolute shift in frequency dependency of the baseline. In other words, the actual coupling between the coils is being changed only slightly by the presence of other materials, but it is enough (in some cases) to affect the ability to measure the sensor signal.

Also, it has been observed that the shifts in baseline are due to the shifts in the impedance between the magnetic field generated by the transmission coils and the field sensed by the pickup coils and not due to other radiating magnetic field from electronic equipment in the nearby environment. This is verified by monitoring the pickup coil 
signal while not driving any current in the transmit coils. There were no shifts in the pickup coil signal as long as no signal was driven on the transmission coils.

Finally, it has been confirmed that the presence of tissue (such as ground beef, as described in Chapters 3 and 4) and small metal objects (such as stents) does not cause attenuation of the sensor signal, even when the presence of said materials shifts the baseline coupling between the transmit and receive coil. This result is to be expected because the wavelengths of the electromagnetic fields generated are much longer than the length scales of interest in the interrogation setup. Fields with such long wavelengths are not appreciably attenuated over short distances.

\subsection{Summary and Performance Goals}

The biliary stent monitoring system consists of four components: the stent itself, the magnetoelastic sensor, the biasing magnet, and the interrogation module. Each component presents specific design challenges. The stent should perform similarly to commercially available stents while also facilitating integration with the sensor and biasing magnet. The magnetoelastic response of the sensor should be tailored to provide sufficient signal amplitude and resonant frequency sensitivity while loaded by bile and sludge. The biasing magnet should provide a uniform biasing field with sufficient strength while minimally interfering with stent operation. The interrogation module should provide a sufficiently strong interrogating field that is oriented with the implanted sensor, while also coupling well with the resulting flux from the sensor. The 
interrogation module must also be configured for appropriate external use, while accommodating reasonable misalignment with the implanted sensor.

The general overview of the design space presented in this chapter, along with knowledge of the application as determined through literature surveys and discussions with physicians, leads to a set of key performance goals as detailed in Table 2-4. The list here is not exhaustive; rather it represents the key performance parameters that will be used to evaluate the effectiveness of specific devices presented in the remainder of this work.

Table 2-4: Desired component performance

\begin{tabular}{|c|c|}
\hline $\begin{array}{l}\text { System Component and } \\
\text { Performance Category }\end{array}$ & Performance Goal \\
\hline \multicolumn{2}{|l|}{ - Stent } \\
\hline Bending Flexibility & $\begin{array}{l}<25 \% \text { lumen loss when bent } \\
\text { around } 5 \mathrm{~cm} \text { diameter }\end{array}$ \\
\hline Radial Stiffness & $\begin{array}{l}0.1-0.5 \mathrm{~N} / \mathrm{mm} \text { measured at ends } \\
\text { and middle of stent length }\end{array}$ \\
\hline $\begin{array}{l}\text { Size (Expanded Diameter, } \\
\text { Length) }\end{array}$ & $\mathrm{D}=4-8 \mathrm{~mm} ; \mathrm{L}=50-100 \mathrm{~mm}$ \\
\hline $\begin{array}{l}\text { Self-Expansion (Expanded } \\
\text { Diameter/Delivery } \\
\text { Diameter) }\end{array}$ & At least 3:1 (200\% expansion) \\
\hline \multicolumn{2}{|l|}{ - Sensor } \\
\hline Full Scale Range & Down to $2 \mathrm{~mm}$ of patent diameter \\
\hline Resolution & $2 \mathrm{~mm}$ of diameter patency \\
\hline Compressibility/Expandability & $\begin{array}{l}\text { Survive compression into } 2.5 \mathrm{~mm} \\
\text { diameter delivery catheter }\end{array}$ \\
\hline $\begin{array}{l}\text { Longitudinal Curvature } \\
\text { Accommodated }\end{array}$ & $\begin{array}{l}70 \mathrm{~mm} \text { or smaller curvature } \\
\text { radius with no change to } \\
\text { amplitude or frequency }\end{array}$ \\
\hline $\begin{array}{l}\text { Signal Change After } 12 \text { Months } \\
\text { Simulated Implantation }\end{array}$ & $\begin{array}{l}<0.5 \% \mathrm{f}_{0} \\
<20 \% \text { Amplitude }\end{array}$ \\
\hline \multicolumn{2}{|l|}{ - Bias Magnets } \\
\hline $\begin{array}{l}\text { Bias Field Provided over } \\
\text { Sensor Length }\end{array}$ & Up to $5 \mathrm{Oe}$ \\
\hline $\begin{array}{l}\text { Change in Stent } \\
\text { Stiffness/Expandability }\end{array}$ & No significant changes \\
\hline \multicolumn{2}{|l|}{ - Interrogation Module } \\
\hline Signal-to-Noise Ratio & $\begin{array}{l}\text { At least } 4 \text { at Full Scale Range of } \\
\text { sensor }\end{array}$ \\
\hline Integration Time & $<10$ seconds \\
\hline $\begin{array}{l}\text { Accommodation of } \\
\text { Interrogation-Module-to- } \\
\text { Sensor Misalignment }\end{array}$ & $\begin{array}{l}+/-5 \mathrm{~cm} \text { in lateral directions with } \\
80 \% \text { of signal amplitude and no } \\
\text { change in resonant frequency; at } \\
\text { least } 7.5 \mathrm{~cm} \text { implant depth }\end{array}$ \\
\hline
\end{tabular}




\section{Chapter 3}

\section{First-Generation System - Ribbon Sensor with Hybrid Magnetic Stent}

The first-generation system consists of discrete neodymium magnets that bias a ribbon sensor attached to the stent. Figure 3-1 illustrates a conceptual sketch of the system. The work presented in this chapter is intended to address key challenges associated with wireless magnetoelastic monitoring of biliary stents, including component fabrication, system integration, and proof-of-concept testing and signal measurement. The stent used in this work is a custom design, with features added to facilitate system integration. More detail on the magnet, stent, and sensor components is in the following sections.

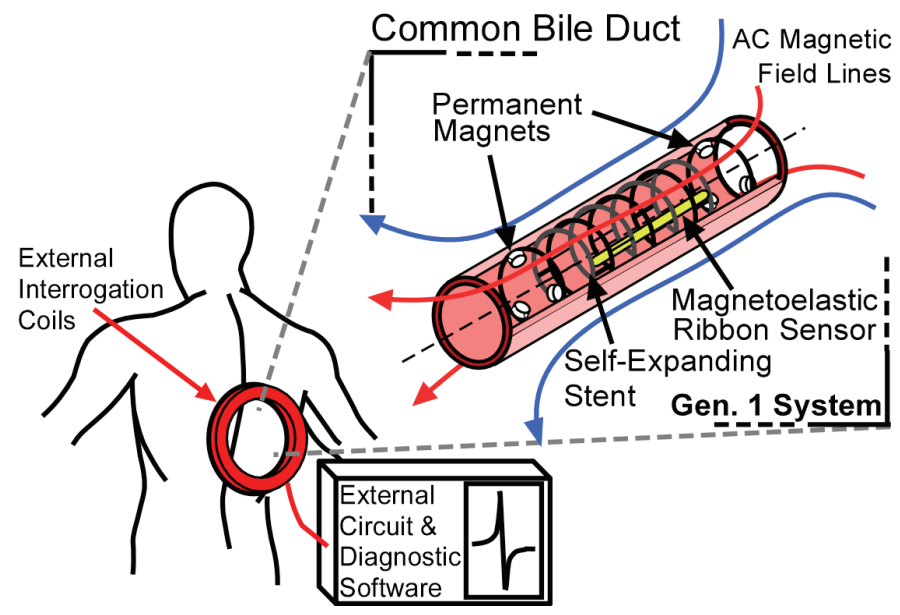

Fig. 3-1: Conceptual sketch of the first-generation system. A ribbon sensor is integrated along the sidewall of the stent. Permanent magnets are also integrated with the stent, biasing the sensor. External coils can be used to interrogate the sensor wirelessly. 


\subsection{Design and Fabrication}

\subsubsection{Stents}

As described in section 2.1, most commercial self-expanding stents (e.g., those offered by Boston Scientific) rely on elastic expansion of a braided metal mesh. The mesh consists of 10-15 small diameter $(\sim 100 \mu \mathrm{m})$ wires that are braided together in a tubular shape. This manufacturing process adds challenge to the inclusion of features that allow the attachment of sensors for enhanced functionality. An investigation into alternative stent fabrication approaches is thus warranted. Previous work has shown the feasibility of micro-electro-discharge machining ( $\mu \mathrm{EDM})$ of cardiac stents in a planar manufacturing approach [Tak06, Tak04]. In this biliary stent system, a planar approach to fabrication facilitates the inclusion of mechanical features for sensor attachment and stent seam closure. Manufacturing the stent as one piece also removes the exposed wires in commercial devices that sometimes lead to duct perforation. To this end, the stents used in this work are batch-fabricated using photochemical machining (PCM). By utilizing the photochemical machining process, the designer is granted freedom to include assembly points at which the sensor can be attached. The sensor attachment points in this work take the form of hooks (Fig. 3-2A). Prior to attaching the sensor, the hooks are folded up so they stand out of plane. In this manner, the hooks act as not only an attachment point, but also as a stand-off that provides an operating gap between the sensor and the stent sidewall.

The stents are batch fabricated from planar $316 \mathrm{~L}$ stainless steel foil. The basic pattern of the stent is a diamond mesh, similar to the mesh formed after braiding filaments in the commercial design. The side length of each diamond is nominally $1 \mathrm{~mm}$. 
PCM, or chemical blanking, is a process that utilizes a laser-defined mask to lithographically pattern a photoresist (PR) layer covering the base metal layer [ASM89]. The PR is developed and the unprotected metal is etched with a heated spray of etchant. In this way, intricately patterned flat metal parts can be produced, with thicknesses ranging from $10 \mu \mathrm{m}$ to $1.6 \mathrm{~mm}$. The base metal sheet is typically $300 \times 450 \mathrm{~mm}$, although larger sheets can easily be processed. A typical PCM process flow is shown in Figure 3-3.
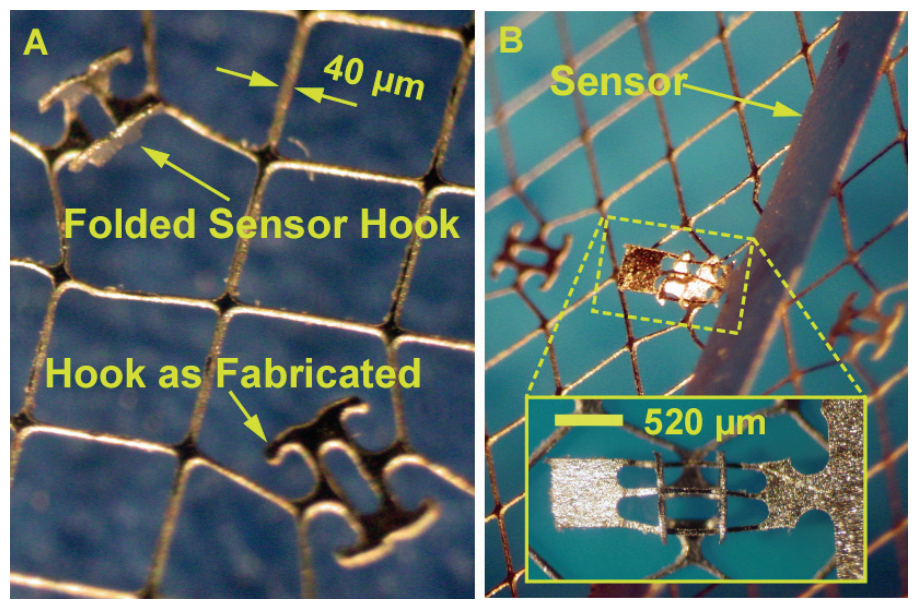

Fig. 3-2: A) Integrated sensor attachment points before and after folding out of the plane of the stent sidewall. B) Assembled sensor and stent at midlength. The attachment points also serve as standoffs to minimize contact-associated signal degradation.

PCM is derived from printed circuit board etching processes, so the most common photoresists and etchants are geared towards processing copper. However, with appropriate surface treatment (to promote photoresist adhesion) and modified etchants, many other metals can be processed. The photoresist is typically laminated onto both sides of the base metal layer, and both sides are exposed simultaneously. Typical etchants include ferric chloride, cupric chloride, sodium hydroxide, hydrochloric acid, or 
even hydrofluoric acid for especially chemically resistant metals. Among others, copper, aluminum, stainless steel, nickel alloys, platinum, tungsten, and even titanium can be processed with an appropriate etchant. The etching is an isotropic process, so masks are adjusted for undercut based on the thickness of the part. Feature sizes are constrained by the workpiece thickness, and the minimum feature size is approximately equal to the thickness. For a $50 \mu \mathrm{m}$ thick foil as used in this work, lateral feature sizes are as small as $33 \mu \mathrm{m}$, with external radii as small as $22 \mu \mathrm{m}$. Tolerances of $+/-10 \%$ of the part thickness are typical. Material removal rates are approximately $10-50 \mu \mathrm{m} / \mathrm{min}$, although looser tolerances can allow for faster etching.

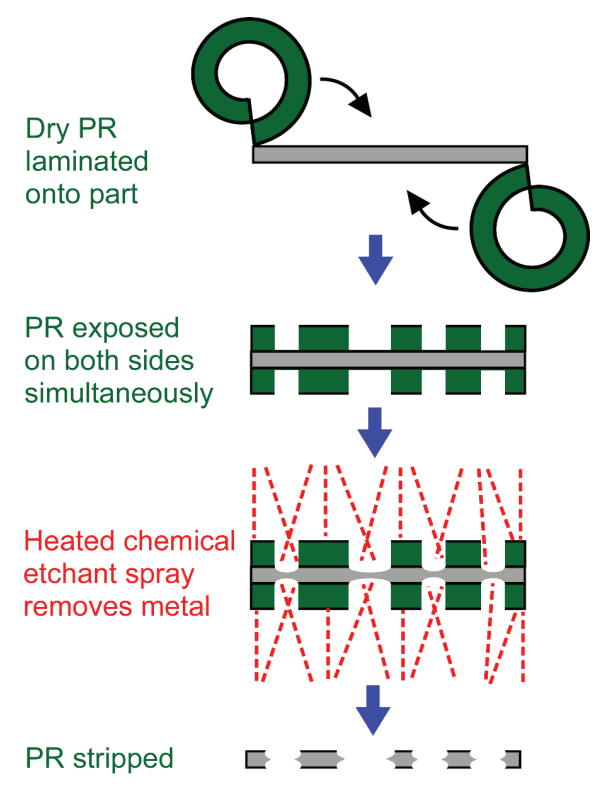

Fig. 3-3: Photochemical machining (PCM) process flow.

The main advantages of PCM include the ability of burr-free processing of thin metal without distortion or other physical changes to the workpiece. Additionally, very hard and brittle metals are machined without difficulty. Of importance to this work, 
magnetically soft materials can be processed with PCM while retaining optimal permeability - indicating that PCM may be an option for processing the magnetoelastic sensor material. Chief disadvantages of PCM include the limitations imposed by the chemistry of the workpiece, as well as the fact that sharp radii cannot be produced due to the undercut involved in the isotropic process.

\subsubsection{Permanent Magnets}

The permanent magnets must be integrated in such a way as to provide a magnetic field of sufficient uniformity and magnitude to properly bias the sensor, yet also to minimally interfere with the expansion of the stent and allow an open lumen to be maintained. A conceptual schematic of an integration scheme is shown in Fig. 3-4. For the $37.5 \mathrm{~mm}$ long by $2 \mathrm{~mm}$ wide sensors used in this study, a bias field strength of 2.5-3.5 Oe is necessary for optimal signal amplitude. A finite element simulation was performed (Ansoft Maxwell 11.1) using two circumferential rings of six $0.8 \mathrm{~mm}$ thick by $1.6 \mathrm{~mm}$ diameter neodymium magnets (K\&J Magnetics). Simulation results show that this configuration can achieve sufficient field strength and uniformity for the needs of this design (Fig. 3-5).

As sensor length is reduced, the required bias field strength for optimal signal increases slightly. However, because a reduction in sensor length allows the circumferential rings of permanent magnets to be spaced more closely together along the length of the stent, a slightly stronger field can be provided by smaller magnets. For instance, a reduction in sensor length from $37.5 \mathrm{~mm}$ to $12.5 \mathrm{~mm}$ allows a reduction in magnet thickness from $800 \mu \mathrm{m}$ to $400 \mu \mathrm{m}$. The thickness of the magnets was controlled 
by utilizing a lapping procedure with a diamond slurry (Fig. 3-6). The permanent magnets can be attached to appropriate portions of the stent using a biocompatible adhesive or epoxy. For this preliminary work, a quick-setting epoxy was used to affix the magnets.

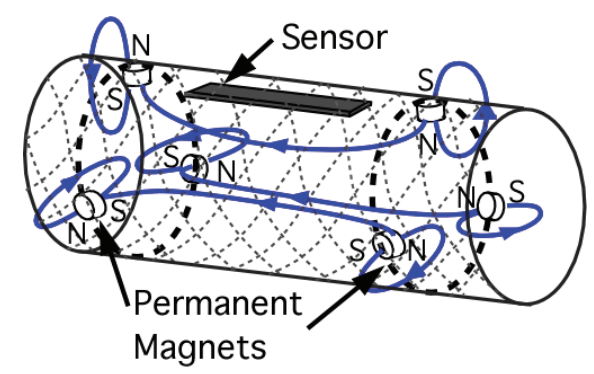

Fig. 3-4: Conceptual schematic of in-stent permanent magnet configuration and resulting field lines. The magnets are incorporated in the stent sidewall to maintain an open lumen. The magnets are located outside of the length of the sensor so that the sensor does not attach to the magnets during the stent expansion process. By orienting similar poles toward the center of the stent at either magnet ring location, stent expansion is unhindered by mutual attraction.

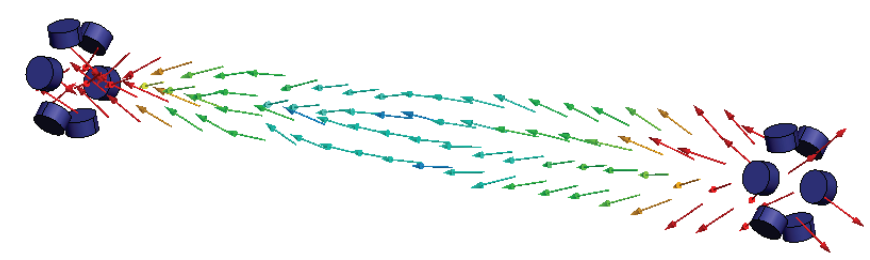

Fig. 3-5: Magnetostatic FEA shows a uniform field of $\sim 3$ Oe directed along the length of the sensor with this magnet configuration.

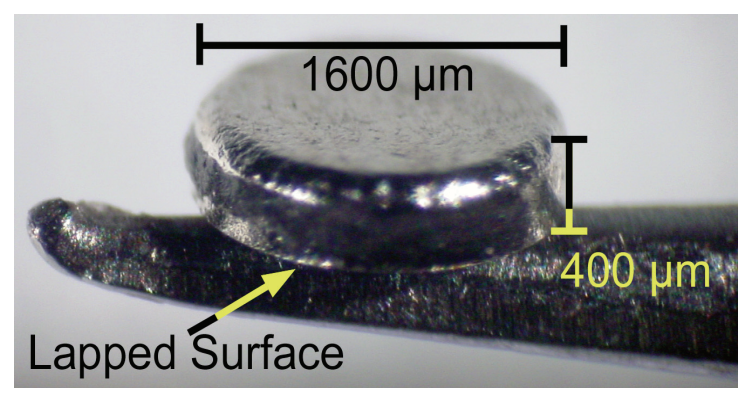

Fig. 3-6: Neodymium magnet lapped from $800 \mu \mathrm{m}$ to $400 \mu \mathrm{m}$, on tip of tweezers. 


\subsubsection{Ribbon Sensors}

The magnetoelastic sensors are patterned from $28 \mu \mathrm{m}$ thick planar Ni/Fe alloy foil (Metglas ${ }^{\mathrm{TM}} 2826 \mathrm{MB}$ ) by $\mu \mathrm{EDM}$. The basic sensor shape is a strip (ribbon) of constant cross-section. The attachment feature on the sensor is an integrated clip at the ribbon mid-length. This clip is fabricated at the same time as the sensor using either serial or batch-mode $\mu$ EDM [Tak02]. In this work, the clip consists of three beams coupled at the ends (Fig. 3-2B). This clip is aligned over the hooks on the stent and then brought into engagement with the hooks. As the engagement length increases, the outer beams are deflected over the hooks and eventually snap elastically back into place under the hooks. The middle beam remains on top of the hook and keeps the other beams engaged with the hooks. This arrangement keeps the clip and sensor at the top of the hook and away from the stent sidewall, as desired.

\subsubsection{Final Assembly}

The sensor is attached and the permanent magnets are affixed while the stent remains in a planar state. In order to prop open the bile duct as intended, the planar stent must be shaped into a tube. The photochemical machining process allows for deformable mechanical features to be placed on the lateral edges of the planar stent. When the stent is rolled into a tubular shape, these mechanical features can be interlocked such that the tubular shape is maintained (Fig. 3-7). The mechanical features remain inside the tubular profile of the stent so they do no damage to the duct wall. The stent in its final assembly state is shown in Fig. 3-8. 


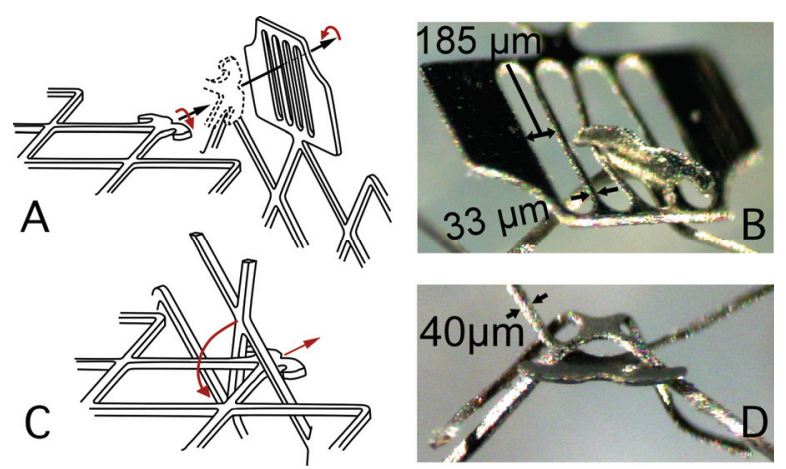

Fig. 3-7: A, B) Conceptual drawing and image of seam closure method 1 . The hook is rotated 90 degrees and inserted through the slot. The hook returns to its as-fabricated orientation and resists disassembly. C, D) Conceptual drawing and image of seam closure method 2. The mesh on the opposite side of the seam acts as an alignment feature for the hook. The tines are then folded over.

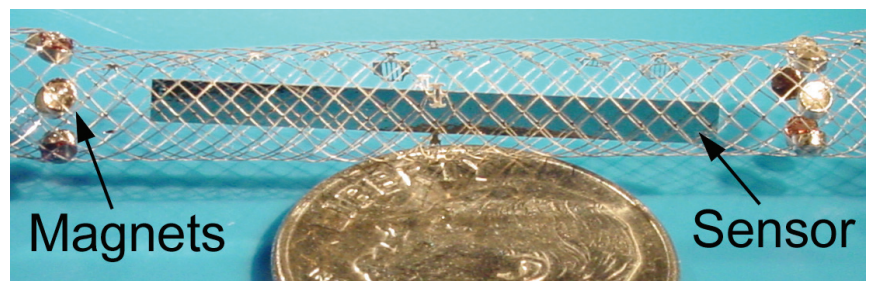

Fig. 3-8: Fully-assembled device, shown with a US dime. The stent is $5.5 \mathrm{~mm}$ in diameter. This sensor is $25 \times 2 \mathrm{~mm}^{2}, 28 \mu \mathrm{m}$ thick. The 12 neodymium magnets are 800 $\mu \mathrm{m}$ thick and $1.6 \mathrm{~mm}$ in diameter and attached with epoxy.

\subsection{Experimental Methods}

The field strength of the magnet configuration is measured to ensure the designed bias field is being supplied. An Analog Devices Hall probe (AD22151) gives an estimate of the field strength, although the probe does average over a relatively large $(0.5 \mathrm{~mm}$ by $0.5 \mathrm{~mm}$ ) active area that is located well away from the edges of the probe. For the firstgeneration system, this method is sufficient to get a clear picture of the field in the sensor area, as the sensor is located in a uniform portion of the field resulting from the discrete magnets. 
Bile viscosity changes are precursors to sludge accumulation [Jun01, Zha02, Ko03], so sensor response to viscosity was evaluated. The system was tested over a range of viscosities by placing the system in a glass vial $(2.5 \mathrm{~cm}$ inner diameter) filled with water with varying sucrose content [Lid04]. The glass vial was then placed in the appropriate test setup. The system was tested in the extracorporeal setup (B) (as described in section 2.4) both with and without a $7.5 \mathrm{~cm}$ thick package of bovine tissue surrounding the system. The bovine tissue was intended to mimic the intervening tissue of a patient in which the system had been implanted and to help assess any signal degradation due to such tissue.

As described in section 2.2, accumulation of sludge results in a mass-loading effect on the sensor. For bench-top testing purposes, this process is simulated by the application of paraffin to the $2.5 \mathrm{~mm} \times 37.5 \mathrm{~mm}$ ribbon sensor using a dip-coating method. The buildup of paraffin presented a reasonable non-uniformity in mass distribution, which is important because sludge accumulation in situ is not likely to be perfectly uniform. Again, the loaded system was tested in a fluid medium in the extracorporeal configuration (B) (as described in section 2.4) with the bovine tissue package in place.

Important mechanical properties of the stent were also tested. To test the retention force of the sensor attachment features, three sensors were attached to three different attachment points on three different stents. The stents were held fixed while a tensile load was applied to the sensor at the mid-length of the sensor. The tensile load was measured with a load cell (Imada, Inc.) and peak values were recorded. The local radial flexibility of the stent was measured by applying a compressive load across the diameter 
of the stent with the load cell tip. The stent was first oriented with the seam towards the load cell tip, and then testing was repeated with the seam oriented $90^{\circ}$ from the load cell tip.

\subsection{Results}

Viscosities spanning those of bile with varying sludge content are from 1-14 cP [Jun01], so the viscosity of the test fluid was varied over most of this range using an aqueous sucrose solution as described previously. As shown in Figure 3-9, the resonant frequency of the magnetoelastic sensors typically shifted by a total of about $2.8 \%$, or 1.7 $\mathrm{kHz}$. This demonstrates sensitivity to viscosity shifts that precede sludge accumulation. This also highlights the importance of having an integrated magnetic field with a constant orientation; as described previously, the $\Delta \mathrm{E}$ effect can easily result in a $2 \%$ frequency shift with a $45^{\circ}$ change in field-to-sensor orientation, which would wash out frequency changes due to viscosity.

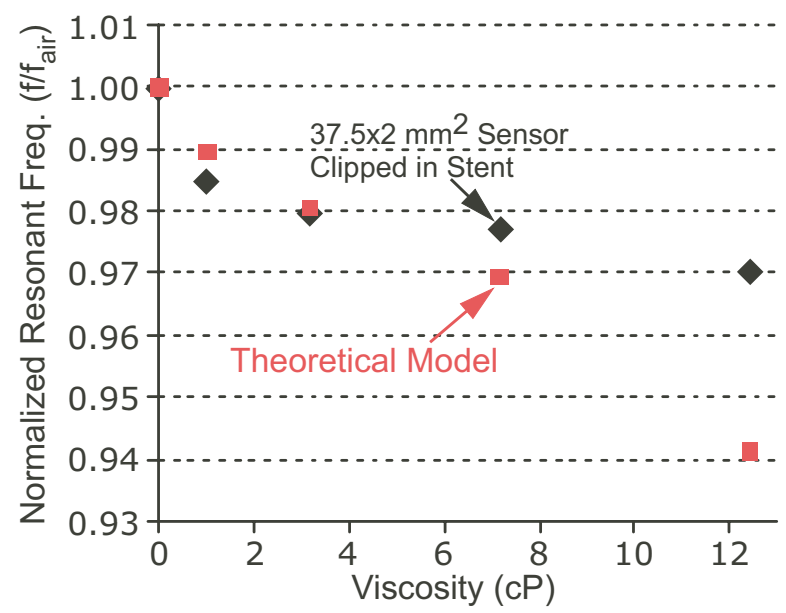

Fig. 3-9: Normalized resonant frequency response to viscosity changes. 
The system was also tested for response to mass loads by successively coating the sensor in paraffin (Fig. 3-10). The resonant frequency shifts by $24 \mathrm{kHz}$, or $40.7 \%$, after a mass of $45 \mathrm{mg}$ has been added, in very good agreement with the analytical model proposed in section 2.2.2. This mass load represents 2.5 times the unloaded mass of the sensor. If the mass is assumed to be distributed in a uniform thickness on the sensor and if the sludge is also uniformly distributed on the stent with the same thickness, then this mass load would represent a significant $29.7 \%$ occlusion of a $4 \mathrm{~mm}$ diameter stent due to sludge accumulation. Additionally, it can be seen from Figure 3-10 that the frequency sensitivity of the sensor has not saturated at this mass load, so the full scale range of the sensor figures to be even greater than that reached in this test. This will be discussed further in sections 4.4.1 when more extensive results from isolated (non-integrated) ribbon sensors are presented.

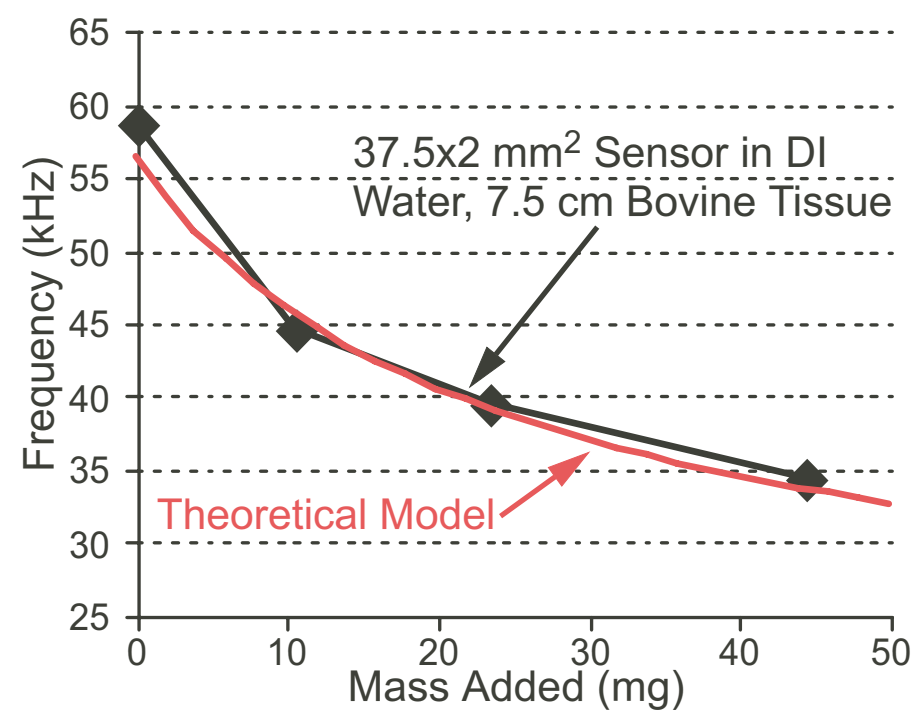

Fig. 3-10: Resonant frequency response to paraffin mass loads. 
Other important results for this system include the magnetic field delivered by the integrated neodymium magnets, as well as some mechanical properties of the stent and sensor attachment features. For two rings of six $1.6 \mathrm{~mm}$ diameter by $800 \mathrm{~mm}$ thick neodymium magnets separated by $45 \mathrm{~mm}$, the field measured with the Hall probe was approximately 3 Oe. At the sensor attachment point, the minimum tensile force withstood for the three samples was $0.367 \mathrm{~N}$. The radial stiffness of the stent measured between $0.145-0.2 \mathrm{~N} / \mathrm{mm}$, with the lower values associated with applying the load directly to the seam. The radial stiffness is similar to that measured for the commercial stent, even though the stent for this system was fabricated from $50 \mu \mathrm{m}$ thick foil while the commercial stent is fabricated from $100 \mu \mathrm{m}$ diameter filaments.

\subsection{Discussion}

Work on this first generation system addresses one of the possible sources of error in wireless magnetoelastic stent monitoring by integrating the biasing magnetic field with the stent. The work also represents a preliminary investigation into the potential for batch fabrication of biliary stents. More importantly, it also motivates investigation for further improvements in each of three main components of the system - the stent, the sensor, and the permanent magnets.

This work utilized stents fabricated from $316 \mathrm{~L}$ stainless steel in a pattern that mimicked the open mesh of commercially available braided stents. This fabrication method results in simple integration of mechanical sensor attachment features, as well as a one piece stent design that eliminates exposed wires that are present in common 
commercial designs. However, the one piece design does lack the elastic recovery capability afforded by the non-connected nature of the braided filament commercial design. Specifically, it was found during testing that the elastic recovery of this stent design - on the order of $2 \%$ - is well below that required for self-expanding biliary stents. This issue can possibly be addressed by using a material with higher yield strain, such as Elgiloy $^{\mathrm{TM}}$, as well as by altering the stent mesh pattern to avoid areas where large plastic strain occurs.

The permanent magnet integration scheme presented in this work, although effectively biasing a sensor for usable results, also provides opportunity for improvement. The adhesive bond between the permanent magnets and the stent may be prone to failure. In addition, the magnets are still too bulky in comparison to the required feature sizes for minimally-invasive delivery of the expanding stent. Specifically, attempting to compress one or more of the $1.6 \mathrm{~mm}$ diameter magnets into a typical $1.3-2.5 \mathrm{~mm}$ diameter delivery catheter would likely result in a crushed magnet or a ruined delivery catheter.

Similarly, the $2.5 \mathrm{~mm} \times 37.5 \mathrm{~mm}$ ribbon sensor provides a very usable signal, sensitivity and full-scale range when loaded with paraffin. However, the width and length of the sensor are at the high end of acceptably fitting inside typical delivery catheters and stent lengths, respectively. Additionally, the mechanical sensor attachment method used in this work, although illustrative of a potential integration technique, is likely not robust enough to withstand all of the rigors of implantation and indwelling, presenting a risk of the sensor breaking away from the stent.

In summary, batch fabricated planar biliary stents allow for integration of magnetoelastic sensors and permanent magnets for wireless monitoring of direct 
indicators of restenosis. Paraffin mass loads up to $45 \mathrm{mg}$ simulated tissue growth on 37.5 $\mathrm{mm} \times 2 \mathrm{~mm}, 28 \mu \mathrm{m}$ thick sensors, resulting in a $24 \mathrm{kHz}$ resonant frequency shift. As viscosity is varied from that of healthy bile to that of diseased bile, a resonant frequency shift of $1.7 \mathrm{kHz}$ was measured. These preliminary results show promise and warrant investigation into further improvements as will be discussed in the remainder of this work. 


\title{
Chapter 4
}

\section{Second-Generation System - Patterned Sensor and Conformal Magnetic Layer}

\begin{abstract}
Although the first-generation system is shown to be effective in bench-top testing (as described in Chapter 3), the discrete approach to the magnet and sensor components is but one design option. Another design approach is to use distributed components to maintain important distributed flexibility of the implant. Components that conform to or mimic the open, flexible structure of the stent would lead to a system that is able to withstand and accommodate the deformations required during catheter-based delivery, as well as lead to a system that preserves the structural functionality of the stent. With this viewpoint, the second-generation system integrates with the stent a sensor and biasing permanent magnet layer that conform to the meshed topology and tubular curvature of a biliary stent (Fig. 4-1). Further, the impact of structural patterning, sensor shaping, and sensor material optimization through thermal treatments is investigated. Additionally, designs for batch-patterned self-expanding stents are presented, with expandability far exceeding that of the stent design described in Chapter 3.
\end{abstract}




\subsection{Design}

Again, a focus is placed on the design of the sensor, DC biasing magnets, and the self-expanding stent for this second generation system. The interrogation components described in section 2.4 are sufficient to make bench-top performance comparisons for new sensor and biasing magnet designs.

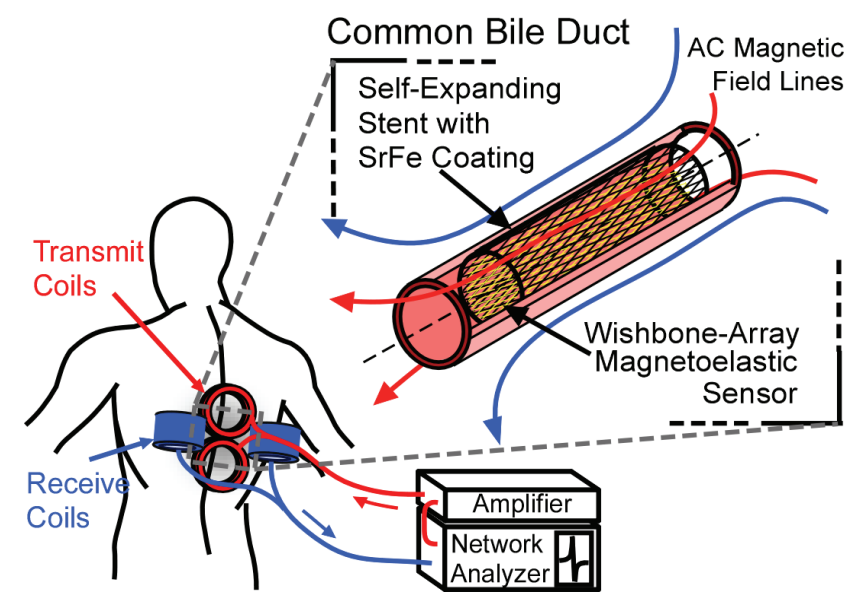

Fig. 4-1: Conceptual sketch of the second-generation system. A patterned magnetoelastic sensor is integrated along the sidewall of a self-expanding biliary stent. The stent is coated with a conformal magnetic layer that biases the sensor. The sensor can be wirelessly interrogated with external coils.

\subsubsection{Wishbone-Array Sensor}

As has been discussed, biliary stents generally reach their final in situ diameter via an elastic self-expansion. This is in opposition to the plastic expansion of typical balloonassisted cardiac stents. The need for large elastic diameter recovery in biliary stents leads to not only the utilization of materials with superior elastic properties (e.g. chrome-nickel Elgiloy $^{\mathrm{TM}}$ or nickel-titanium $\mathrm{Nitinol}^{\mathrm{TM}}$ ) but also to the use of open diamond-shaped patterns. As has been pointed out, often these patterns are formed by braiding filaments 
into a tubular shape.

In keeping with the philosophy of mimicking the design of the stent with the design of the magnetoelastic sensor, it is desirable to use a material with superior elastic properties and to shape the material in diamond-shaped patterns. Fortunately, Metglas ${ }^{\mathrm{TM}}$ alloys are materials with excellent magnetostrictive properties as well as excellent elastic properties. For instance, the $2826 \mathrm{MB}$ alloy as used in this work is reported to have a yield strain of $1.6 \%$ [Lin95], which is even higher than most cold-reduced Elgiloy ${ }^{\mathrm{TM}}$ yield strains of $\sim 1 \%$ [Elg08]. Metglas ${ }^{\mathrm{TM}}$ is not, however, readily available in filament form. It is also likely that a resonant sensor fashioned from braided filaments would have low structural coupling and high damping at braid cross-over points, limiting efficiency as a resonator. For these reasons, both the stent and sensor in this work are batchfabricated from foils of different base materials utilizing photochemical machining as described in section 3.1.2. As shown in Figure 4-2, an elongated wishbone-array pattern is used; this pattern allows good mechanical flexibility for both the stent and sensor while maintaining mechanical coupling and minimizing joint damping for efficient resonant operation of the sensor. To ensure that plastic strain in the sensor was avoided during deformation that is required during catheter-based delivery, an FEA model was utilized (Fig. 4-3). With the fabricated dimensions, FEA suggests that the wishbone-array sensor can undergo a 50\% reduction in circumference without plastic strain that may result in degradation of sensor performance.

The coupled magnetomechanical FEA model presented in section 2.2.3 was also used to analyze the complex wishbone-array structure. Reasonable agreement in predicted and experimentally measured resonant frequency was shown (within $11-20 \%$ of 
experimentally measured values, depending on the mode shapes). Trends in the experimentally measured amplitude and phase are also predicted by the FEA (Fig. 4-4). Discrepancies between experimentally measured values and predicted values may be due to differences in how well the biasing field penetrates the smaller feature sizes of the wishbone-array (thus resulting in a different operating point for the material), or may be due to slightly different damping properties for the transverse movements that exist in the wishbone-array mode shapes versus those for the purely longitudinal modes of the ribbon sensors.

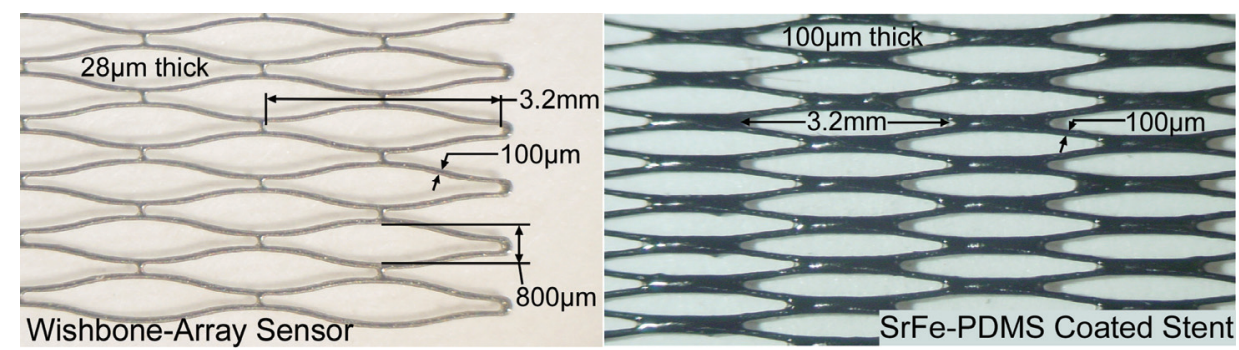

Fig. 4-2: LEFT: A portion of a wishbone-array sensor, along with important dimensions. RIGHT: Stent (fully-connected design) with SrFe-PDMS coating.

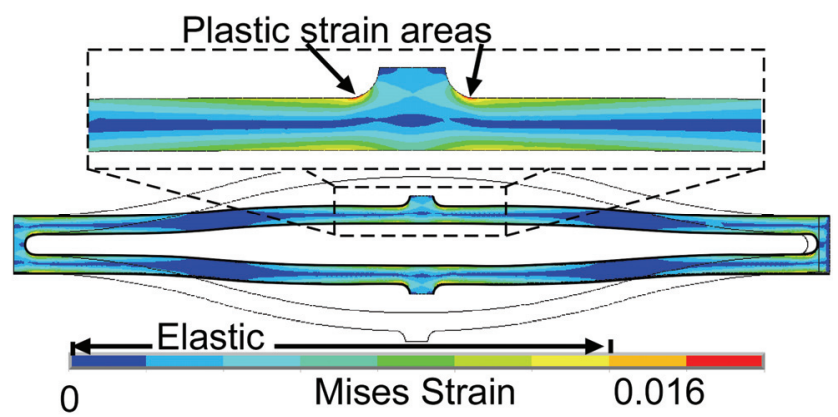

Fig. 4-3: FEA calculated strain in a single wishbone cell during cell deformation. The deformation shown corresponds to that required for a reduction in diameter of $50 \%$ for the curved array. As can be seen, only a small area of the wishbone structure exceeds the yield strain with this deformation. 

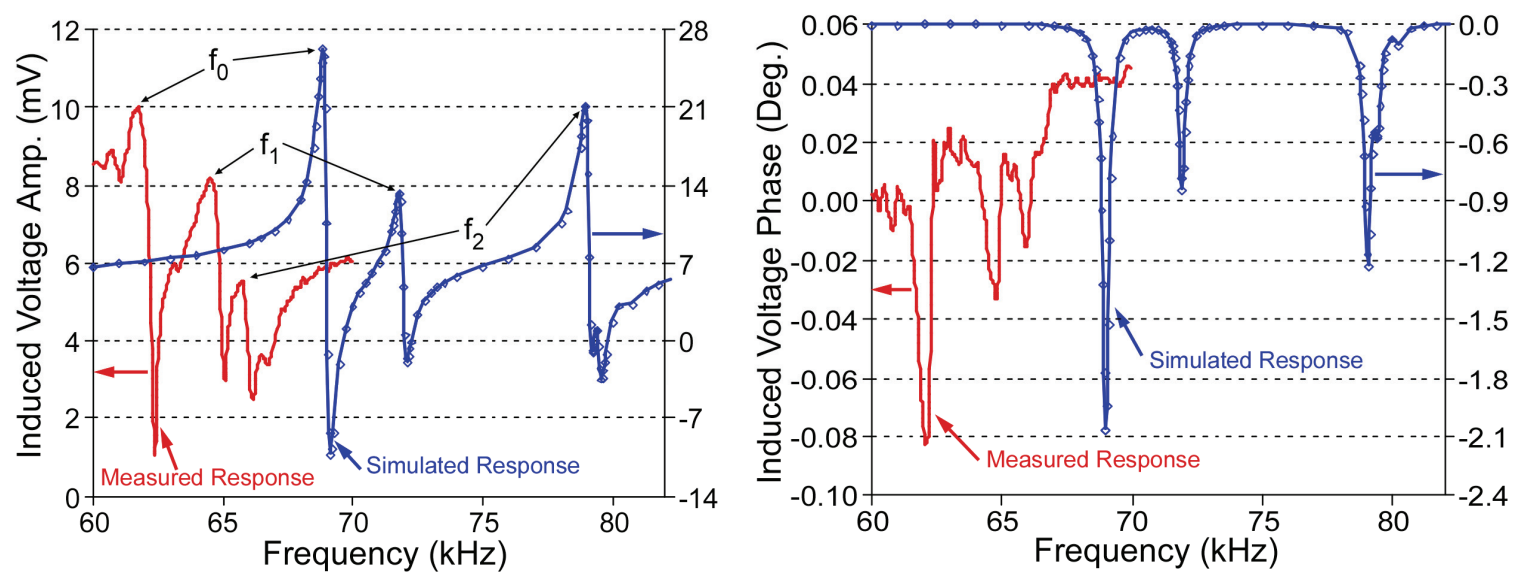

Fig. 4-4: FEA calculated and experimentally measured amplitude (right) and phase (left) frequency response for an unloaded planar wishbone-array sensor. The amplitude and phase scales for each curve are different to enhance visibility.

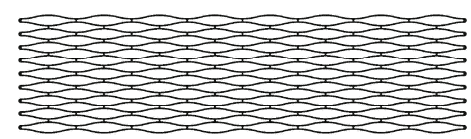

Undeformed

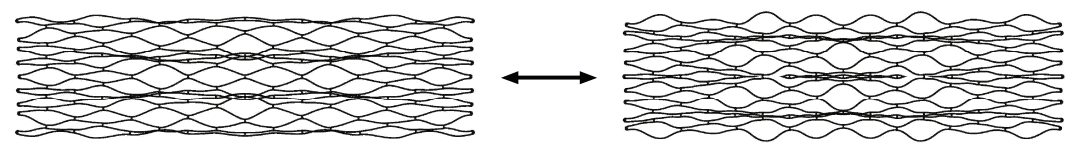

$68.85 \mathrm{kHz}$

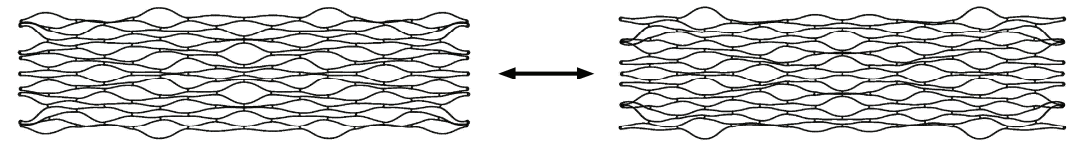

$71.8 \mathrm{kHz}$

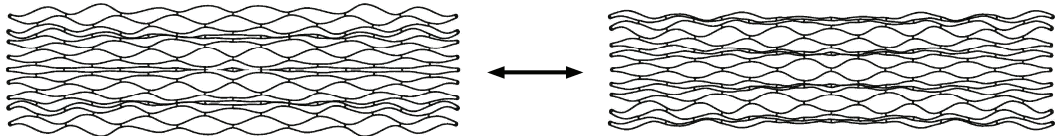

$78.95 \mathrm{kHz}$

Fig. 4-5: FEA calculated mode shapes for a planar wishbone-array magnetoelastic sensor, occurring at the listed (predicted) resonant frequencies. Note the difference between these shapes and the purely extensional mode of ribbon sensors.

In Fig. 4-5, calculated mode shapes for planar wishbone-array sensors are shown. The mode shapes displayed are at frequencies corresponding to peaks in the calculated frequency response for the planar wishbone-array sensors, with the mode shape at 68.85 $\mathrm{kHz}$ resulting in the largest response amplitude. Note that the mode shapes combine 
significant longitudinal and transverse motion, whereas mode shapes of traditional ribbon sensors are limited to longitudinal motion. It is expected that the sensor may possess some unique properties due to the transverse motion of the mode shapes and large effective surface area as compared to ribbon sensors discussed in Chapter 3.

Because the stent application calls for a generally tubular shape, and the lateral dimension of the sensor is larger than the diameter of the stent, the sensor must be curved into a tubular or semi-tubular shape to best match the stent geometry. The tubular curvature can be potentially added in a number of ways. Mechanical supports may cause the sensor to curve around the inner wall of the stent. The curvature could be set with sufficient mechanical stress. Alternatively, high temperatures and a support fixture may be used to shape the material while imparting minimal residual stress. The method used should result in minimal degradation in the performance of the resonating sensor. This is discussed further in section 4.2.1.

\subsubsection{Conformal Magnetic Layer}

To achieve optimal magnetomechanical coupling, the magnetoelastic material must be biased with a DC magnetic field. This field offsets the as-fabricated anisotropy of the magnetic domains in the material, and the optimal field is dependent not only on the material of the sensor, but also on the feature sizes or aspect ratio of the final sensor. The process of selecting a bias field magnitude can be considered analogous to selecting an operating point for a transistor in an electrical circuit.

The previous work with integrated discrete magnets (Chapter 3) showed that sensor performance is improved when the bias field is as uniform as possible. This uniformity is 
difficult to achieve with integrated discrete magnets, because the field strength will necessarily decay as the distance from the magnets increases. However, if the magnetized portion of the system were to be continuously distributed, the field strength could be maintained more uniformly. This improves the sensor performance and eradicates high magnetic field gradients that lead to undesirable magnetic forces.

The distributed magnet is chosen in this work to be a layer of strontium ferrite ( $\mathrm{SrFe})$ particles $(\sim 1 \quad \mu \mathrm{m}$ average diameter, Hoosier Magnetics $)$ suspended in polydimethylsiloxane (PDMS, Sylgard 184, Dow Corning). This choice is made again in keeping with minimally altering the functionality and structure of the biliary stent with the additional components. In this case, the polymer-suspended particles can be applied in a thin, flexible layer conforming exactly to the stent structure (Fig. 4-2, right).

Other polymers have been used as a base for SrFe particles in microfabricated magnets described elsewhere [Lag97]. SrFe particles have the advantages over other magnetizable candidate materials of being chemically inert (owing to their ceramic nature), and of being widely and inexpensively available in very small particle sizes. The chemical inertness is especially valuable in our implantable application. PDMS is chosen in this work due to its generally accepted biocompatibility and due to processing ease. In fact, the entire polymer-suspended magnet fabrication process (as described later) is preferable in terms of ease compared with alternative options such as sputtering or electrodeposition of a thin-film magnetic layer. 


\subsubsection{Batch-Patterned Self-Expanding Stent}

In this work, the batch-patterning of self-expanding biliary stents utilizing a photochemical machining (PCM) process (see section 3.1.1) is again investigated. This fabrication process results in a stent that displays structural continuity similar to that available from laser-machined tubes while adding the advantages of economic batch production and potential integration of other planar structures (such as sensors or circuitry). To improve the elasticity of the stent, chrome-nickel Elgiloy ${ }^{\mathrm{TM}}$ is used in this work, which is a material used in commercially available stents. This material has a much higher yield strain than stainless steel $\left(\sim 1 \%\right.$ for Elgiloy ${ }^{\mathrm{TM}}$ versus $\sim .15 \%$ for $316 \mathrm{~L}$ stainless steel [ASM89]), which is a material commonly used in cardiac stents and is the material used in the design described in Chapter 3. The need for large elastic diameter recovery in biliary stents leads to not only the utilization of materials with superior elastic properties but also to the use of open diamond-shaped patterns that close when the stent is compressed into the delivery catheter. As shown in Figure 4-2, an elongated wishbone-array pattern is used; this pattern allows high elastic expandability for the stent while also maintaining adequate radial rigidity. Bilinear elastic-plastic finite element analysis predicts that this pattern will result in a stent that can elastically increase diameter by $200 \%$, similar to commercially available stents.

In an attempt to improve the bending flexibility over the fully-connected wishbonearray pattern shown in Fig. 4-2, an alternative design is also investigated as shown in Fig. 4-6. For this switchback design, linkages separate the wishbone-array columns or bands of the pattern. The location of the linkages is varied from band to band so that, in the final tubular form of the stent, all bending axes have similar flexibility. 
The stent is patterned in a planar state, but must adopt a tubular shape for implantation. To accomplish this, a long wire is integrated along one long side of the planar pattern, while open loops are placed on the other long side of the pattern and adjacent to the long wire. The wire can then be threaded through the loops to close the resulting seam. The wire can be locked in place by looping back onto itself and bonding or welding. Alternatively, the seam edges can be bonded together using a biocompatible adhesive. These processes are illustrated further in section 4.2.4.

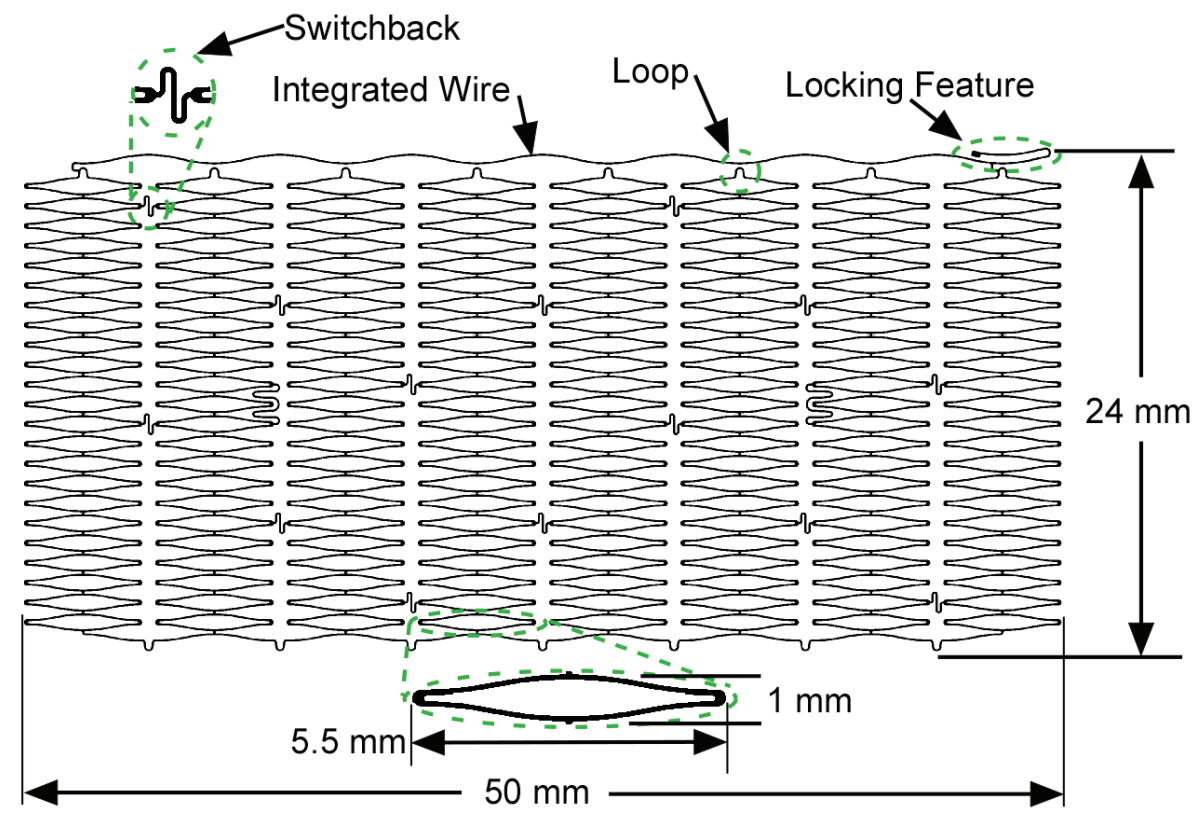

Fig. 4-6: Switchback stent design layout with important dimensions.

\subsection{Fabrication}

\subsubsection{Wishbone-Array Sensor}

The wishbone-array sensors for this work are batch fabricated from a $28 \mu \mathrm{m}$ thick foil of $2826 \mathrm{MB}$ Metglas ${ }^{\mathrm{TM}}$ utilizing the PCM process. Feature sizes of the individual struts 
are $75-100 \mu \mathrm{m}$, which is near the minimum feature size for the technology. The overall size of the active portion of the sensor (not including the anchor areas discussed later) is $7.5 \mathrm{~mm} \times 29 \mathrm{~mm}$, with a mass of $9.1 \mathrm{mg}$. Lateral portions of the wishbone-array sensor are connected to the active area with single struts. These areas act as anchors, and the single struts isolate the vibrating active area from the anchors.

PCM is a planar process, so the as-fabricated sensors are also planar. Initial attempts to add curvature to the sensor via mechanical stress proved catastrophic for the sensor signal. Instead, the tubular shape is achieved in this work by placing the sensor against the inner wall of a fixture tube and annealing at a high temperature for 30 minutes. Various final radii can be achieved by either changing the radius of the fixture tube or by changing the anneal temperature. For instance, a $4.6 \mathrm{~mm}$ radius results from annealing at $375^{\circ} \mathrm{C}$ for 30 minutes inside a $3.6 \mathrm{~mm}$ radius tube, while a $1.6 \mathrm{~mm}$ radius results from annealing inside a $1.25 \mathrm{~mm}$ radius tube (Fig. 4-7). Lower temperatures lead to lesser final curvature.

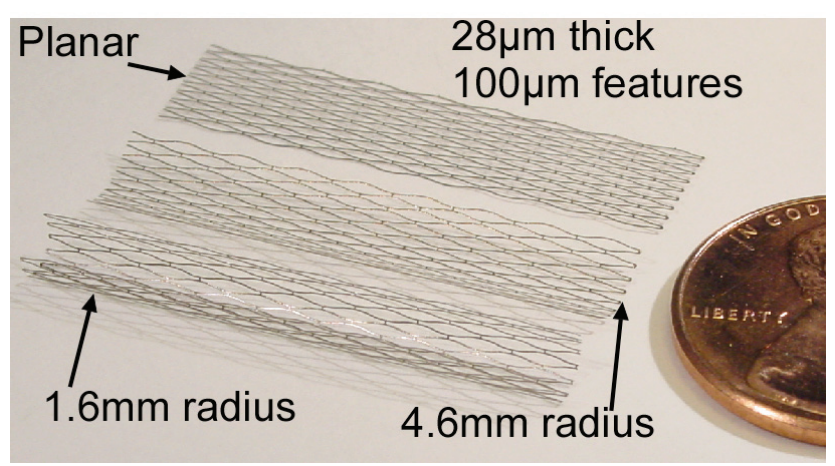

Figure 4-7: Curvature is achieved by placing the sensor inside a tube and annealing at $375^{\circ} \mathrm{C}$ for 30 minutes. Tubes were $\sim 30 \%$ smaller than the final sensor radius. 


\subsubsection{Conformal Magnetic Layer}

To form the conformal magnetic layer, the PDMS is first mixed in a 10:1 base-tocuring-agent ratio. Subsequently, the SrFe particles are introduced in 1:1, 3:1, or 1:3 SrFe-to-PDMS by weight ratios and mixed in by hand until the mixture is consistent (usually about 1 minute of mixing time). The mixture is then poured or spread into a mold containing the stent. The stent is then peeled out of the mold, with a conformal layer of the magnetic suspension adhered. The layer is then cured for 30 minutes at $60^{\circ} \mathrm{C}$. Thicker layers can be built up by repeating the process. Finally, the layer is magnetized uniformly along the long axis of the stent using a bench-top pulse magnetizer. In general, the 1:1 SrFe:PDMS ratio offered the best combination of workability and remnant strength of the ratios tested. The resulting magnetic characteristics of $1: 1$ and $2: 1$ SrFe:PDMS are discussed further in section 4.4.2.

\subsubsection{Stent}

The stent is also batch fabricated using the PCM process, in this case from an Elgiloy $^{\mathrm{TM}}$ foil $(100 \mu \mathrm{m}$ thick for the fully connected design, $125 \mu \mathrm{m}$ thick for the switchback design). As intended, the feature sizes and patterns are identical to those of the sensor (Fig. 4-1), with other important dimensions for the switchback design noted in Fig. 4-6. The overall stent sizes are $4 \mathrm{~mm}$ (dia.) $\times 40 \mathrm{~mm}$ long for the fully-connected design, and $6 \mathrm{~mm}$ (dia.) $\times 50 \mathrm{~mm}$ long for the switchback design. 


\subsubsection{System Assembly}

The sensor anchors are bonded to the stent with a thin layer of PDMS. Subsequently, the stent is rolled into a tubular shape and the resulting seam where the edges of the stent adjoin is mechanically joined. This joining process is achieved with two methods in this work. In one method, the edges are brought into alignment with a fixture that also acts as a mold. PDMS is poured into the mold and cured, encasing the seam edges (Fig. 4-8). In the other method, a wire is formed in the pattern on one edge of the stent. The wire is then woven through the loops on both edges to join the seam (Fig. 4-9). The entire assembly process is shown in Figure 4-10, and assemblies are shown in Figure 4-11.

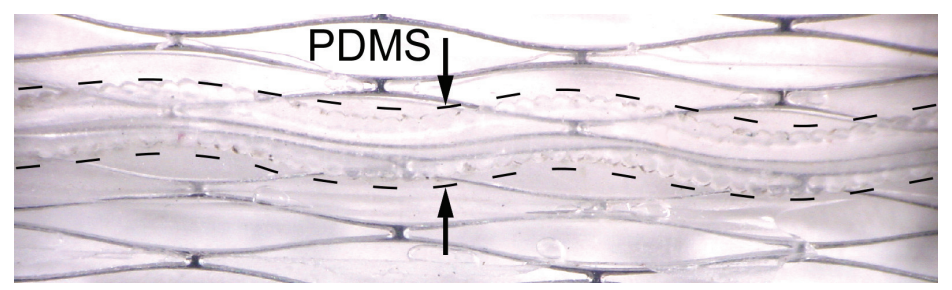

Figure 4-8: The edges of the stent seam can be brought into alignment in a fixture, and subsequently encased in a layer of PDMS (the outline of which is denoted by the dashed lines).

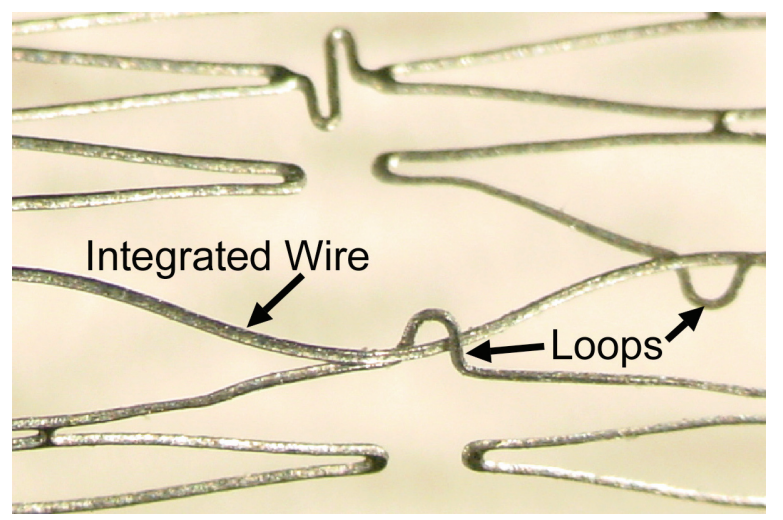

Fig. 4-9: An alternative seam closure method involves threading the integrated wire through the loops on both sides of the seam. Once threaded through, the integrated wire can be locked by bonding closed the locking feature. 


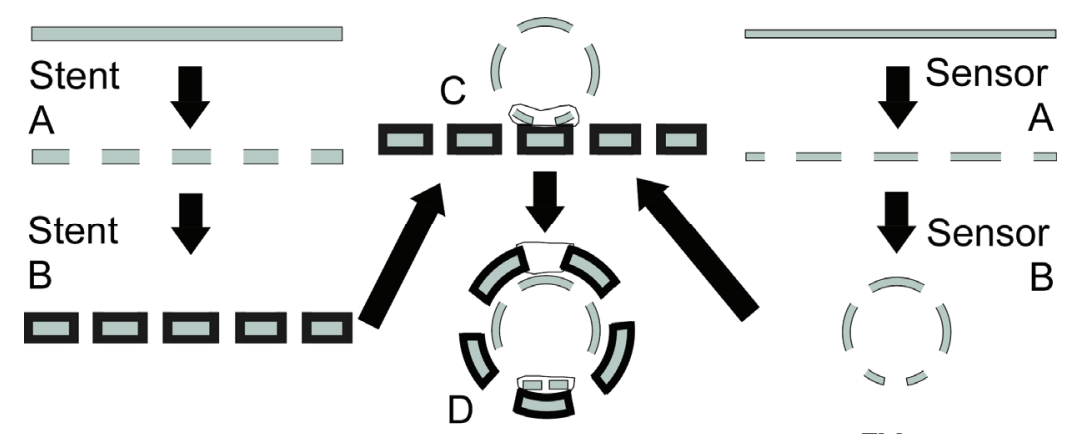

Fig. 4-10: Fabrication process. A) PCM patterning of Elgiloy ${ }^{\mathrm{TM}}$ (stent) and Metglas ${ }^{\mathrm{TM}}$ (sensor). B) Stent coated in SrFe-PDMS layer and magnetized. Sensor annealed in a tube. C) Sensor anchors bonded to stent with PDMS. D) Stent seam joined by bonding or weaving integrated wire.

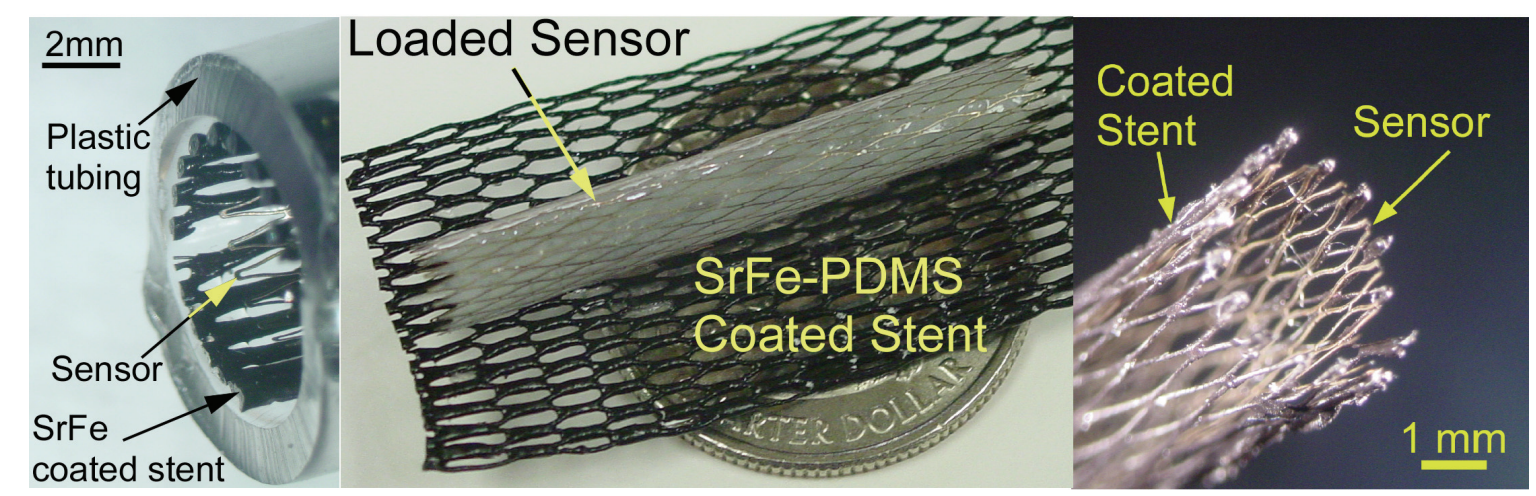

Fig. 4-11: LEFT: Assembled sensor, stent, and magnet layer, inside a plastic tube. The sensor is positioned along the inner sidewall of the stent. MIDDLE: Assembled sensor, stent, and magnet layer. The stent seam is not bonded here for clarity. The sensor has been loaded with layers of acrylate terpolymer. RIGHT: Assembled sensor and coated stent. The coating on this device is thinner $(50 \mu \mathrm{m})$ than the coating on the devices in the left and middle photographs $(200 \mu \mathrm{m})$.

\subsection{Experimental Methods}

\subsubsection{Sensors}

As mentioned previously, bile viscosity changes are precursors to sludge accumulation [Jun01, Zha02, Ko03], so sensor response to viscosity is evaluated. The viscosity response of the second generation system (and of isolated sensors, presented in 
section 4.4.1) is tested by using fluids of known viscosities (Dow Corning) in a polycarbonate test vial (1.25 $\mathrm{cm}$ inner diameter).

For bench-top testing purposes, the sludge accumulation process is simulated in this work by the application of two different materials - paraffin and an acrylate terpolymer to as-cast and thermally treated wishbone-array sensors, as well as to $2.5 \mathrm{~mm} \times 37.5 \mathrm{~mm}$ ribbon sensors. The paraffin is applied in the same dip-coating method described in section 3.2. The acrylate terpolymer is commercially available dissolved in a solvent. The mixture is either sprayed onto the sensor, or brushed on. The solvent is then allowed to evaporate for 30 minutes, resulting in a cured layer prior to testing. The application process for both sludge simulants results in a reasonable variation in mass distribution, which allows the variability of the sensor response due to non-uniform sludge accumulation to be quantified. Additionally, the simulants possess different mechanical properties, so variation in sensor performance due to these differences can also be quantified.

\subsubsection{Conformal Magnetic Layer}

The remanent magnetic moment and coercivity is measured on SrFe:PDMS samples with $1: 1$ and 2:1 by-weight ratios using a vibrating sample magnetometer (courtesy of Princeton Measurements Corporation). The samples are $4 \mathrm{~mm}$ discs, each $100 \mu \mathrm{m}$ thick. Two samples of each ratio are measured as a preliminary quantification of process repeatability.

When evaluating the field strength of the as-fabricated magnetic layer, two methods are used. As described in section 3.2, an Analog Devices Hall probe (AD22151) gives an 
estimate of the field strength, although the probe does average over a relatively large ( 0.5 $\mathrm{mm}$ by $0.5 \mathrm{~mm}$ ) area that is located at an appreciable distance from the edge of the probe. For this system, in which the sensor is located very near the magnetic layer, the spatial averaging due to the size of the Hall probe and the fact that the active area cannot be placed in close proximity to the magnetic layer are larger issues. To address this, the second method of evaluating the field strength is to use a small $(1 \mathrm{~cm} \times 2 \mathrm{~mm})$ ribbon sensor, the performance of which has been characterized for various uniform biasing fields. The small sensor can be placed in different locations along the length of the stent and near the magnetic layer, and the resulting frequency and amplitude of the sensor can be correlated - using the performance characterized in a uniform field - with the local field provided by the magnetic layer. In this way, the $\Delta \mathrm{E}$ effect of the material is used to evaluate the field strength and uniformity of the fabricated magnetic layer.

\subsubsection{Stents}

As described in section 2.1, the key specifications for the biliary stent are bending flexibility, radial stiffness, and expandability. The bending flexibility and radial stiffness determine the ability of the stent to conform to the natural bile duct anatomy while maintaining an adequately sized lumen for bile flow. The expandability of the stent determines the diameter of the delivery system and how small the treated stricture can be prior to placing the stent.

The bending flexibility of the stent should be such that the stent can conform to natural bile duct curvature without significant loss of stent lumen, so the method for determining bending flexibility has been established with this in mind. To test the 
performance of the design with regards to bending flexibility, the stent is placed in a clear Tygon tube with the same diameter as the expanded stent (4 or $6 \mathrm{~mm}$, depending on the design). The tube is then wrapped around cylinders of successively smaller diameter, and the resulting stent lumen is measured visually. The tube is much more flexible in bending than the stent, so that the tube does not experience any lumen loss when wrapped around the standard diameter. This testing method is adapted from [Bas03].

For radial stiffness, it is important to realize that the treated vessel or duct is not likely to be perfectly circular in cross-section; nor is the cross-section likely to be identical along the entire treated length (i.e. the stricture can be localized). Additionally, the stiffness of the vessel or duct may also vary along the length of the stent due to pathological tissue changes. For these reasons, it is important for the stent to have appropriate stiffness as determined by a local application of force. Finally, the stiffness should be uniform and not concentrated at the ends or middle of the stent length. The performance of the stent against this specification is tested by placing the stent in a rigid semi-tube with the same diameter as the expanded stent and locally applying a compressive force with the tip of a force gauge (Imada, Inc.). The force gauge is equipped with a blade probe that allows a localized $(5 \mathrm{~mm} \times 1 \mathrm{~mm})$ application of force to best simulate a local bile duct lesion. The resulting deflection of the stent wall is also measured (using a micrometer to advance the probe tip) to calculate stiffness. The stent is probed at the mid-length and end to establish the uniformity of the stiffness. The stent is also tested in two different orientations - with the force applied to the seam or with the force applied $90^{\circ}$ from the seam - to evaluate stiffness changes due to the seam. The testing is applied to uncoated stents, coated stents, stents with a bonded seam, stents with 
a woven seam, and to a commercially available biliary stent (Wallstent ${ }^{\mathrm{TM}}$, Boston Scientific, $4 \mathrm{~mm}$ diameter).

The expandability of a stent is determined by passing the stent through a rigid tube of an appropriate compressed diameter, and subsequently measuring the diameter of the stent after it elastically recovers. Rigid tubes with progressively smaller diameters are used until the stent no longer recovers. It is necessary to pull the stent through the tubes; to accomplish this, fine nylon line is tied through the stent in multiple areas. The nylon line distributes the force required to pull the stent through the tube while minimally interfering mechanically so that only the compressive stresses applied by the tube are important.

\subsection{Results}

\subsubsection{Isolated Sensors}

To evaluate the response of wishbone-array sensors prior to integration, sensors were tested in the coaxial test setup while biased with dual Helmholtz coils as described in section 2.4. As shown in Figure 4-12, initial evaluation of four as-cast planar wishbonearray sensors showed that the frequency and amplitude responses versus bias field were similar across the sensors for all important modes, indicating a repeatable PCM fabrication process.

To evaluate the effects of thermal treatments that are used to give the sensors curvature, the wishbone-array sensors were then thermally treated either above $\left(375^{\circ} \mathrm{C}\right)$ or below $\left(325^{\circ} \mathrm{C}\right)$ the material Curie temperature $\left(353^{\circ} \mathrm{C}\right)$ and either remained planar or 
were given curvature. As shown in Figure 4-13, post-treatment evaluation showed lower optimal biasing field ( $\sim 1.5$ Oe versus 5 Oe pretreatment) and improved signal level (up to $13.5 \mathrm{mVp}$-p versus $9 \mathrm{mVp}$-p pretreatment). This important result shows that thermal treatment facilitates thinner SrFe-PDMS layers, which simplifies fabrication, minimizes system size, and minimizes any concerns about large chronically implanted magnetic fields.

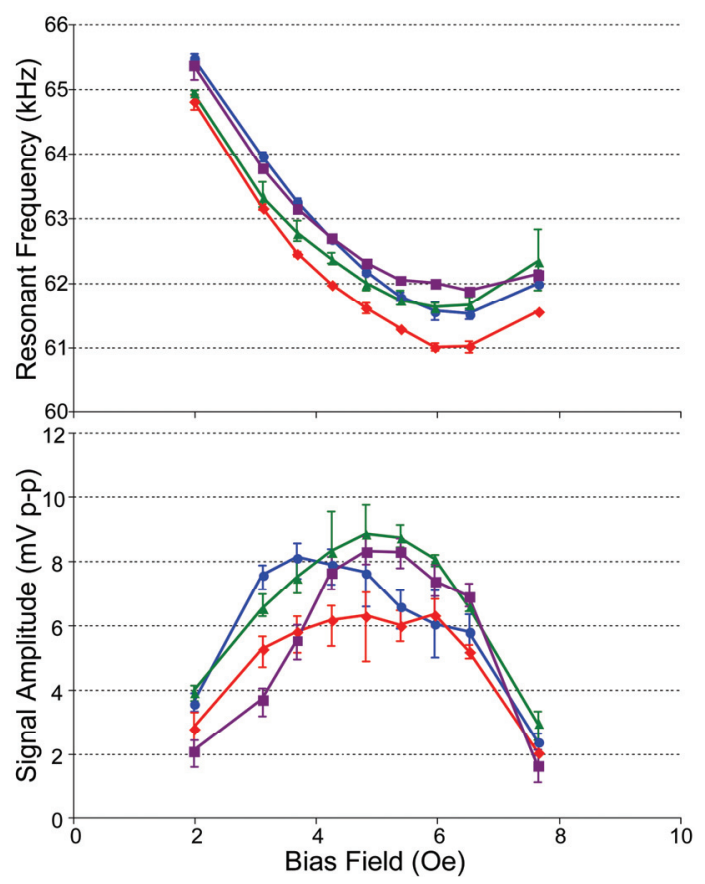

Fig. 4-12: Evaluation of four as-cast planar wishbone-array sensors. The points in these figures represent the mean of three trials, and the error bars represent the minimum and maximum recorded values.

As-cast and thermally treated sensors were compressed through $1.5 \mathrm{~mm}$ diameter tubes - a circumferential deformation of at least $37 \%$ - without signal degradation (Fig. 4-14). The repeatable performance of this test across both as-cast and thermally treated sensors implies that the thermal treatment process does not lead to impaired mechanical 
properties. The slight discrepancy with the FEA model predictions of at least $50 \%$ deformation may be due to an imperfect correlation between the onset of plastic strain and the onset of strains that change the magnetomechanical properties of the material, or may simply be due to the discrete testing points above and below the predicted deformation.

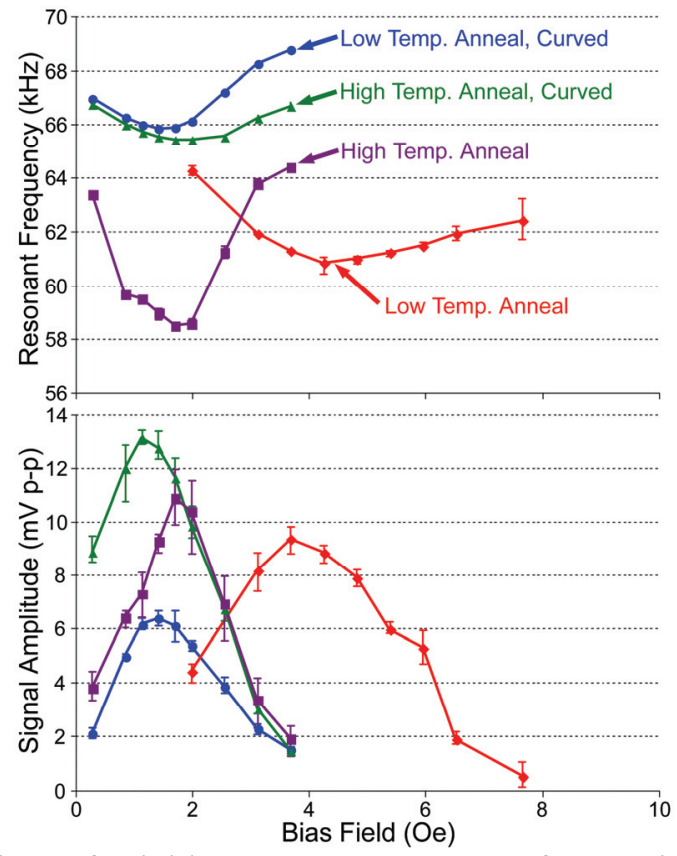

Fig. 4-13: Characterization of wishbone-array sensors after various heat treatments. The color for the curves of specific sensors is unchanged from those used in Fig. 4-12.

Bile viscosity changes are precursors to sludge accumulation, so sensor response to viscosity was evaluated (Fig. 4-15). The tested viscosity range is much greater than the physiological range of bile $(1-12 \mathrm{cP})$, but the results show that sensitivity and signal amplitude is maintained over a very large range that might be suitable for other applications. Note that a $2.5 \mathrm{~mm} \times 37.5 \mathrm{~mm}$ ribbon sensor resonant frequency will drop by only $6 \%$ over this viscosity range. 

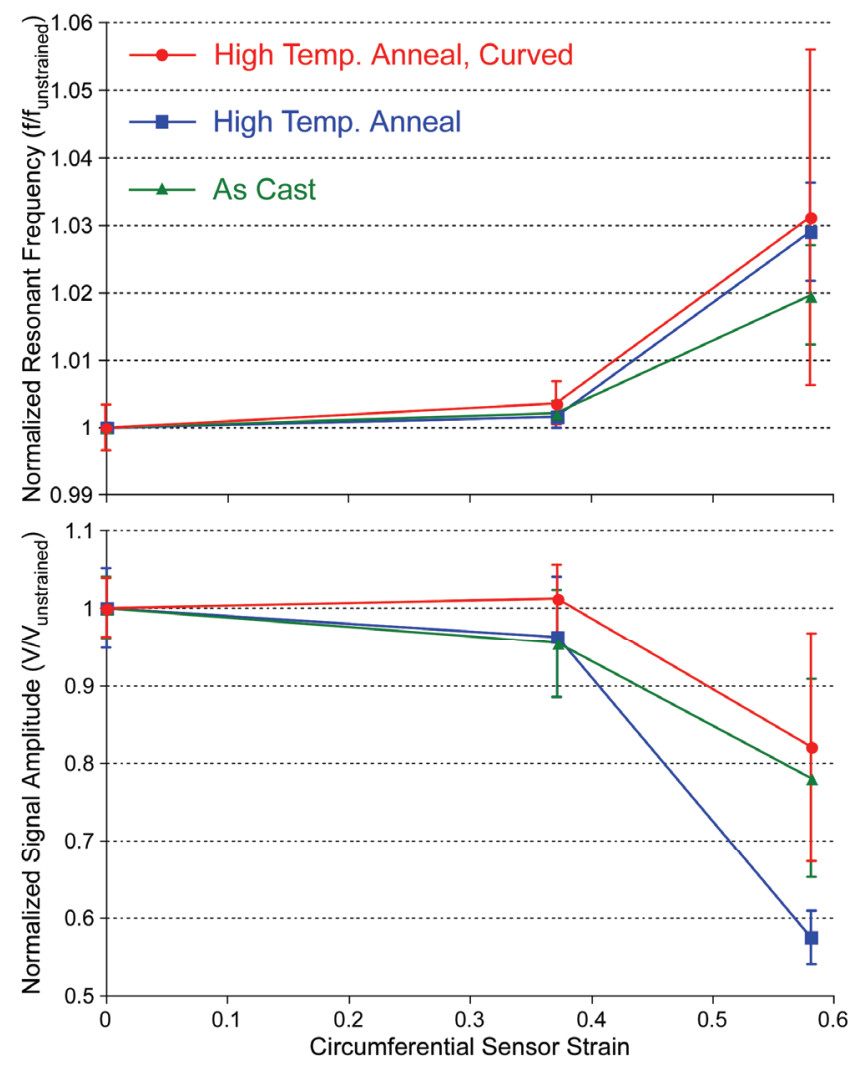

Fig. 4-14: The wishbone-array sensor amplitude and frequency show insignificant changes after strains up to $37 \%$.

As-cast and thermally treated wishbone-array sensors, as well as $2.5 \mathrm{~mm} \times 37.5 \mathrm{~mm}$ ribbon sensors, were evaluated for response to mass loading with both paraffin and acrylate terpolymer. As shown in Figure 4-16, each of the sensor types reacts similarly in terms of resonant frequency to both sludge simulants. The variability in the frequency data is likely due to changes in the distribution of the mass as the sensor was recoated on separate runs. Repeated tests with a loaded sensor showed a signal amplitude repeatability of $10-20 \%$, while the frequency repeatability was $0.2-0.5 \%$. Further, the full scale range of each sensor type extends into the "critical zone". Based on the initial mass of each sensor type, and assuming the sensors are integrated with $4 \mathrm{~mm}$ diameter stents, the critical zone corresponds to at least $50 \%$ occlusion by a uniform layer of sludge. 
Also, note that the initial amplitude of the ribbon sensors is higher than the initial amplitude of the wishbone-array sensors. However, the amplitude of the ribbon sensors decreases nearly linearly with increased mass loads (with a significant slope that is related to the properties of the loading layer), while the amplitude of the wishbone-array sensors seems to quickly decrease to a plateau and hold that level over nearly the entire tested range (although more scatter in the data exists). Note that the analytical model predicts that signal amplitude for a ribbon sensor will be decreased most with mass loads from stiffer materials with a high loss modulus; thus, it may be reasoned that the acrylate terpolymer used in this study is stiffer and has a higher loss modulus than the paraffin.

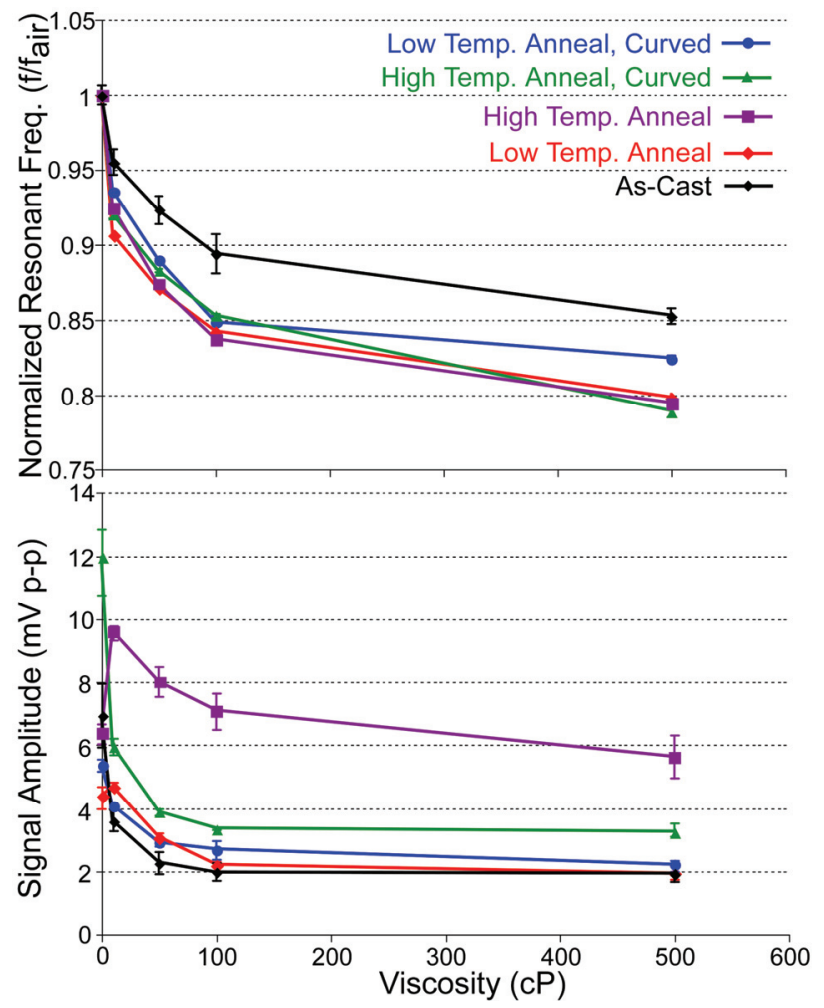

Fig. 4-15: Amplitude and resonant frequency response to viscosity for as-cast and thermally treated wishbone-array sensors. 


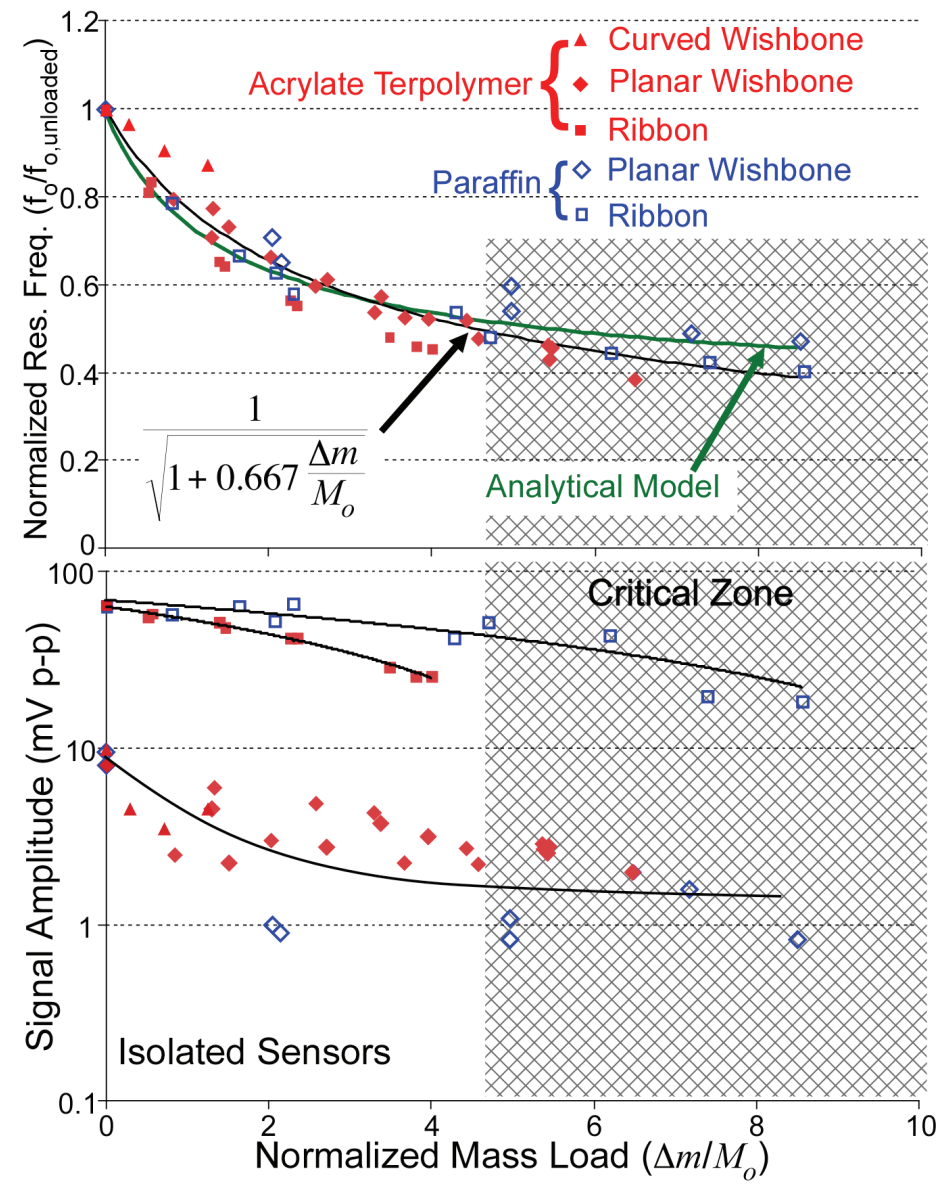

Fig. 4-16: As-cast planar and thermally treated curved wishbone-array sensors, as well as ribbon sensors (2826MB, $2 \mathrm{~mm} \times 37.5 \mathrm{~mm}$ ), were loaded with either paraffin or acrylate terpolymer layers to simulate sludge accumulation. The curve in the top graph is calculated by minimizing the sum of squared errors between all points and the line using an equation of the form shown. Results for the analytical model (utilizing literature values for paraffin properties) are also superimposed. Lines in the bottom graph are guides to the eye. The variability in the resonant frequency for a given normalized mass load is small and likely due to slightly different distributions of the mass.

\subsubsection{Conformal Magnetic Layer}

Typical hysteresis curves measured using the vibrating sample magnetometer are shown in Fig. 4-17. Note that the remanent magnetic moment is proportional to the byweight ratio of SrFe-PDMS in this range of ratios. Additionally, the coercive force is consistent regardless of by-weight ratio. These findings are consistent with previous 
work with polymer-particle composite magnets [Lag97]. The known geometry of the samples, along with the measured hysteresis curves, can be used to estimate the remanent magnetic flux density using an appropriate FEA tool. This value can then be applied to more complicated FEA geometries for predictive design purposes.

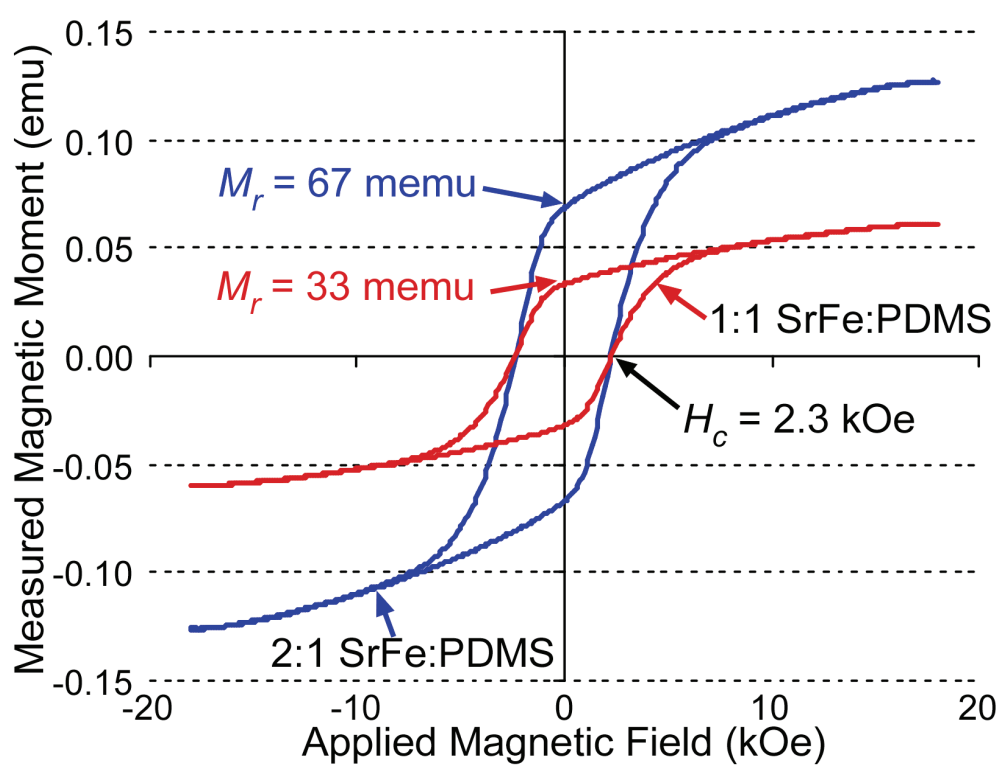

Fig. 4-17: M-H hysteresis curves for $4 \mathrm{~mm}$ diameter, $100 \mu \mathrm{m}$ thick discs of SrFe-PDMS in different ratios. Note that the remanent magnetic moment $\left(\mathrm{M}_{\mathrm{r}}\right)$ is proportional to the particle to polymer ratio.

For the conformal layers applied to stents and used to bias sensors, a number of observations are to be noted. As expected, the resulting biasing field is proportional to the thickness of the coating, which is generally controlled by the number of coats applied. Also, the resulting bias field is stronger for a stent with a smaller diameter assuming all other factors are equal (coating thickness, stent pattern, particle to polymer ratio, etc.). This is also to be expected, since the magnetic material is more densely concentrated for a smaller diameter stent. For a $6 \mathrm{~mm}$ diameter stent, 1:1 SrFe-PDMS ratio, and $100 \mu \mathrm{m}$ thick coating, a bias field strength of approximately 0.5 Oe results. 


\subsubsection{Stents}

A comparison between the mechanical testing results for the stent designs described previously and a commercially available stent is given in Table 4-1. There are three important observations that should be noted. First, the ratio between the expanded and deliverable diameters for the fully-connected and commercial stents are similar $(3 \mathrm{x})$, while the switchback stent ratio is larger $(\sim 4 \mathrm{x})$. This follows from the slightly more elongated wishbone-array pattern in the switchback stent. Figure 4-18 shows the $3 x$ selfexpansion of each stent design. Second, the radial stiffness of the fully-connected stent and the commercial stent are again similar, while the stiffness of the switchback stent is about one-quarter as stiff. The switchback design is less stiff due to the fact that each band of the pattern is only connected to other bands with linkages; these linkages are spaced out and thus the structural continuity is lower. The uniformity of the stiffness varies in a similar fashion amongst the three designs, with the ends being less stiff than the middle. The absolute uniformity of stiffness is best in the switchback design, again indicating that the bands act nearly independently of each other. The alignment between the seam and the force application made insignificant differences in the measured stiffness for the planar batch-patterned designs presented here. Likewise, the method of seam closure made little difference in the radial stiffness. Finally, the fully-connected design performed very poorly in the bending flexibility test, but this shortcoming was ameliorated with the linkages added in the switchback design.

With thin conformal coatings $(\sim 100 \mu \mathrm{m})$ on the fully-connected and switchback designs, the radial stiffness was insignificantly affected. Additionally, the expandability of the designs was also unchanged. However, it was found that the SrFe-PDMS coating 
made passing the stent through smaller tubes much more difficult, requiring a much higher tensile force on the stent to pull the stent through the tube. This is mainly an assembly and delivery issue that should be addressed in future devices; the issue is discussed in more detail in Chapter 6.

Table 4-1: Stent mechanical testing results comparison

\begin{tabular}{|l|l|l|l|}
\cline { 2 - 4 } \multicolumn{1}{c|}{} & \multicolumn{1}{c|}{ Commercial } & Fully-Connected & \multicolumn{1}{c|}{ Switchback } \\
\hline Deliverable Dia. & $1.3 \mathrm{~mm}$ & $1.3 \mathrm{~mm}$ & $1.5 \mathrm{~mm}$ \\
\hline Expanded Dia. & $4 \mathrm{~mm}$ & $4 \mathrm{~mm}$ & $6 \mathrm{~mm}$ \\
\hline Radial Stiffness & $0.07-0.15 \mathrm{~N} / \mathrm{mm}$ & $0.06-0.18 \mathrm{~N} / \mathrm{mm}$ & $0.016-0.04 \mathrm{~N} / \mathrm{mm}$ \\
\hline Bending Flexibility* & $1.6 \mathrm{~cm}$ & $>20 \mathrm{~cm}$ & $3.5 \mathrm{~cm}$ \\
\hline
\end{tabular}

* Measured as the minimum radius of curvature at which $>75 \%$ of the stent lumen remained
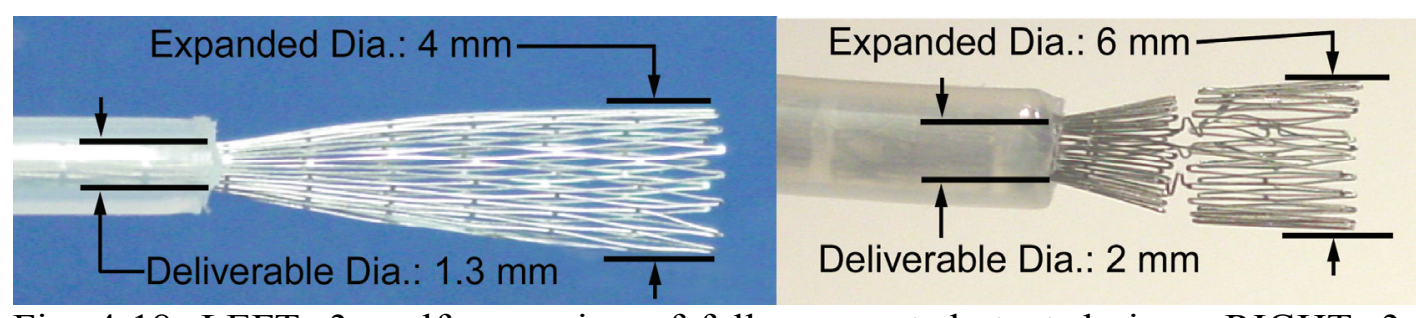

Fig. 4-18: LEFT: 3x self-expansion of fully-connected stent design. RIGHT: 3x selfexpansion of switchback design.

\subsubsection{Integrated System}

As described in section 4.1, the second generation system consists of a curved wishbone-array sensor and a conformal SrFe-PDMS magnetic layer. To closely compare results for the integrated system to the results obtained for the isolated sensors as described in section 4.4.1, the coaxial coil configuration was used. The external dual Helmholtz coils were not used, so the sensor was biased only by the integrated magnetic layer. 
For the second generation system, sensitivity to viscosity over a physiologically appropriate range was measured even as mass was added. This experimental process showed that the normalized frequency response of the sensor to viscosity changes was not significantly affected by initial mass buildup (Fig. 4-19). As shown in Figure 4-19, the resonant frequency of the wishbone-array sensors shifted by about $6.5 \%$ typically over this range of viscosities. Note that the sensitivity over this range is approximately double that of the ribbon sensors. This sensitivity difference is likely due to the different effects that viscous damping has on the transverse motion of the wishbone-array mode shapes. Note that as mass builds on the sensor, the normalized signal amplitude becomes less sensitive to viscosity. This is a trend predicted for the ribbon sensor in the analytical model (refer to Figure 2-4).

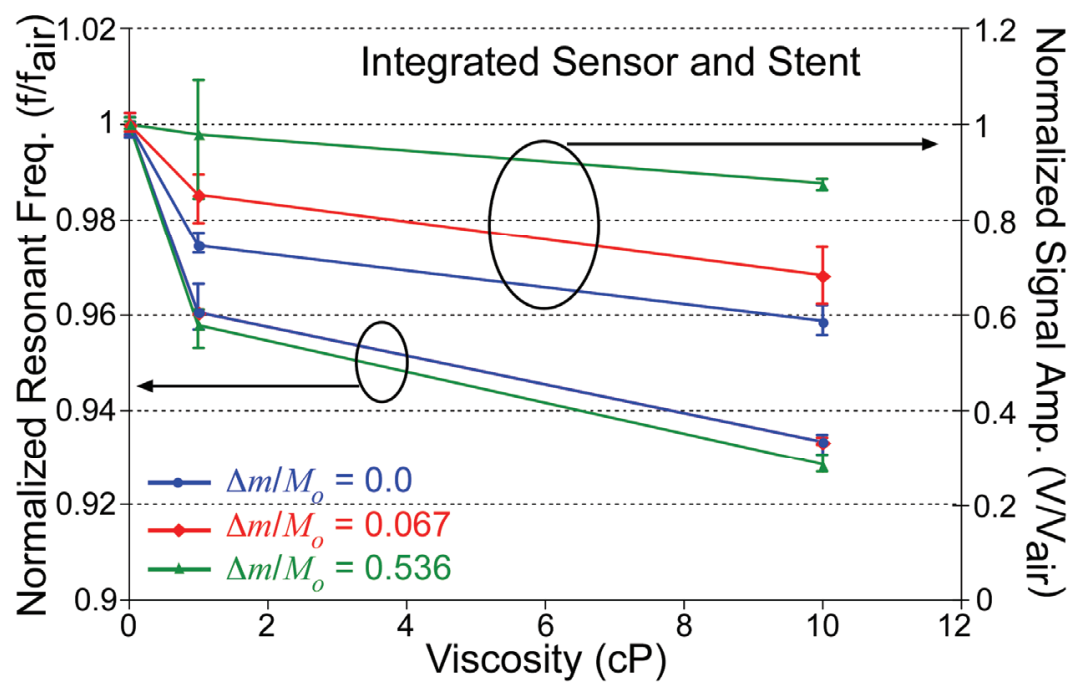

Fig. 4-19: Response of the system to viscosity changes as mass accumulates.

The system was also tested for response to mass loads by successively coating the sensor in acrylate terpolymer. Because of the close integration between the sensor and 
the stent, it was difficult to apply mass directly to the sensor without also applying some to the stent. As such, the actual load on the sensor was back-calculated from the frequency response curve calculated for the isolated sensors (shown in Figure 4-16). Note that the trend seen in Figure 4-16 for the wishbone-array sensors in which the amplitude quickly decreases to a plateau is also seen for the integrated system in Figure $4-20$.

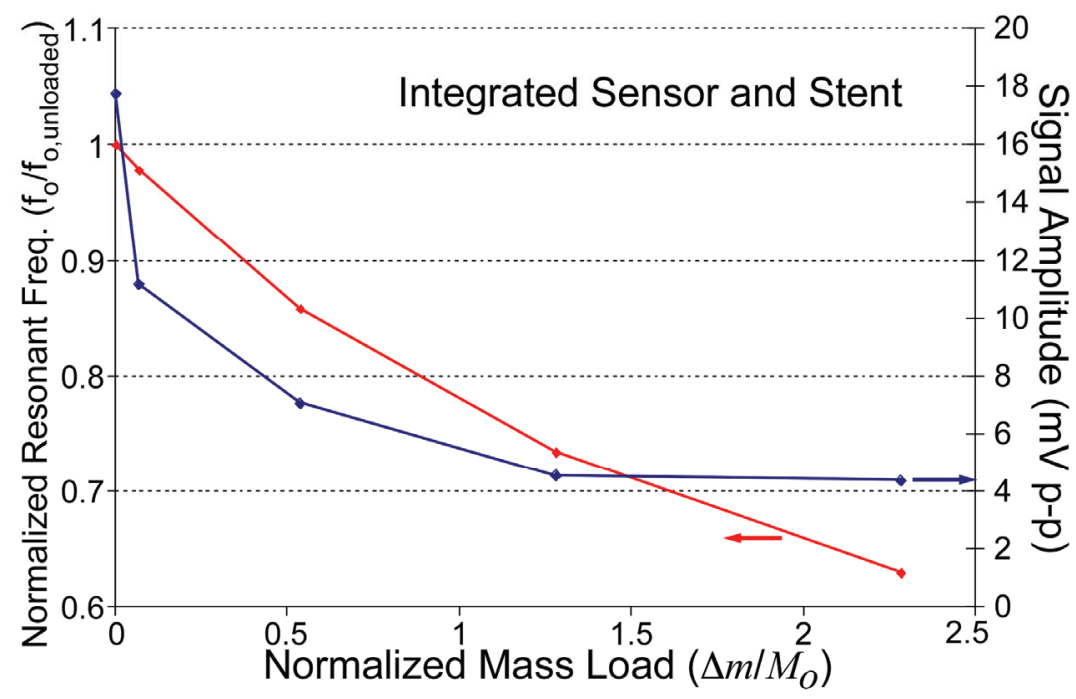

Fig. 4-20: Response of the system to mass buildup. Mass added to the sensor was difficult to separate from mass added to the stent, so the equation for the curve in Fig. 416 was used to back-calculate the mass load.

\subsection{Discussion}

The work presented in this chapter demonstrates the feasibility of using the photochemical machining process to batch-pattern complex resonant magnetoelastic sensors. Although better tolerances or smaller features may perhaps be attainable with other machining processes (e.g. batch-mode micro-electro-discharge machining), the 
photochemical machining process provides an economical and sufficiently repeatable method for quick prototyping and higher volume production of magnetoelastic sensors. The complex patterns that can easily be generated using photochemical machining also can open opportunities for enhanced sensor design and feature integration; this will be discussed further in Chapter 5.

As shown in the experimental results of this chapter, the resonant frequency of magnetoelastic sensors (both ribbon and wishbone-array designs) responds similarly to mass loads of paraffin and acrylate terpolymer. The stiffnesses of these materials are similar, especially compared to the range of stiffnesses encountered in biological materials (from bone to muscle to sludge). The experiments in this chapter show that, in general, the response of the sensors to materials with this stiffness is repeatable, although some variability in the sensor response for a given mass load can exist due to differences in the spatial distribution of the mass. Further, the results in this chapter show that viscosity can affect the resonant frequency even while materials of this stiffness load the sensor. Because the viscosity of the fluid that the duct is carrying can vary even while the material accumulates, a method for separating the effects of viscosity from the effects of mass loading is required for a robust monitoring system. A technique for accomplishing this separation of effects is first discussed in section 2.2.2.2 and further illustrated in the results and discussion of Chapter 5. The repeatability of the response of the sensors to materials with this stiffness is promising in that a measured resonant frequency can be fairly confidently correlated to a given mass load, which can then be mapped to an approximate thickness. Thus, the sensors should be useful for estimating the level of stent occlusion. However, because the stiffness of biological material can vary so greatly, it will be 
important to evaluate the utility of the sensors while loaded with a wider range of materials. This is discussed further in Chapter 5.

Three important advantages of the wishbone-array sensor over typical ribbon sensors in this application are made clear by the work presented in this chapter. First, the fine features sizes and large open area of the pattern present little obstruction to bile flow, which is the primary objective of a biliary stent. Second, the sensors are much more accommodating of the large deformations required for catheter-based delivery. Third, the sensors have a higher sensitivity to viscosity changes, which is a clinically relevant parameter in many pathological conditions. The principal disadvantage of the wishbonearray sensor, at least with the present design, is the smaller signal amplitude. However, preliminary results show that the signal amplitude scales with the overall sensor length, so this disadvantage may be mitigated with a longer sensor design within the limits of acceptable stent lengths.

This work also illustrates the benefits of thermal treatments to give three-dimensional shape to the sensor. With this approach, the appropriate shape is produced while residual stresses are relieved by annealing. This allows the sensor performance to be maintained (and in some cases improved). The bias field strength required for optimal performance is also reduced, facilitating integration of a thin permanent magnet layer.

The work presented in this chapter also demonstrates the potential for batch-patterned self-expanding stents. The use of Elgiloy ${ }^{\mathrm{TM}}$ as a self-expanding stent material is common due to its excellent elasticity; however, the metal is not a likely candidate for photochemical machining due to its anti-corrosive properties. The stents fabricated for the work presented in this chapter show that photochemical machining of Elgiloy ${ }^{\mathrm{TM}}$ is 
feasible, and use of the material - along with focused structural design - enhances the expandability of the device over the devices presented in Chapter 3. In fact, the performance of the designs presented in this chapter testifies to the fact that photochemical machining can be part of a viable process for producing self-expanding biliary stents with mechanical performance similar to that of commercially available stents.

Two seam-closure methods for planar-patterned self-expanding stents presented in this chapter, and add to the methods demonstrated in Chapter 3. From a viewpoint that emphasizes ease of processing and mechanical robustness, the method in which an integrated wire is woven through loops on each side of the seam, described in section 4.2.4, is most promising. The linkages described in the switchback design were very effective at increasing the bending flexibility of the stent. However, a balance must be struck in the number and distribution of the linkages so that the axial strength of the stent is not compromised - a characteristic that is important during the assembly of the stent into the delivery catheter.

In summary, the second-generation system integrates a flexible wishbone-array magnetoelastic sensor and conformal magnetic layer with a self-expanding biliary stent as a wireless system that monitors the stent environment. The system is sensitive to physiologically appropriate viscosity changes, showing a $6.5 \%$ decrease in resonant frequency in $10 \mathrm{cP}$ fluid. The system also is capable of measuring mass buildup that is associated with sludge accumulation, showing a 38\% decrease in the resonant frequency after an applied mass load of $20.9 \mathrm{mg}$, or $2.3 \mathrm{x}$ the mass of the sensor. Techniques for separating the effects of mass from effects of viscosity are discussed further in Chapter 5 . 
The integrated system is robust to deformations required for delivery and provides a uniform biasing layer that minimally affects stent mechanics. Additionally, the improved viscosity sensitivity of the wishbone-array sensor may find use in industrial applications like monitoring oil refinement. 


\section{Chapter 5}

\section{Advanced Sensor Functionality}

The wishbone-array sensor design presented in Chapter 4 demonstrated that photochemical machining (PCM) could be effectively used to produce a sensor with complex geometry that granted additional functionality to the sensor - namely, an ability to conform to the open topology and tubular curvature of the biliary stent, allowing for the flexibility and expandability of the system to be maintained. The work presented in this chapter adopts the advantages of this approach while focusing on sensor designs with added functionality over the basic ribbon and wishbone-array sensors described in Chapters 3 and 4.

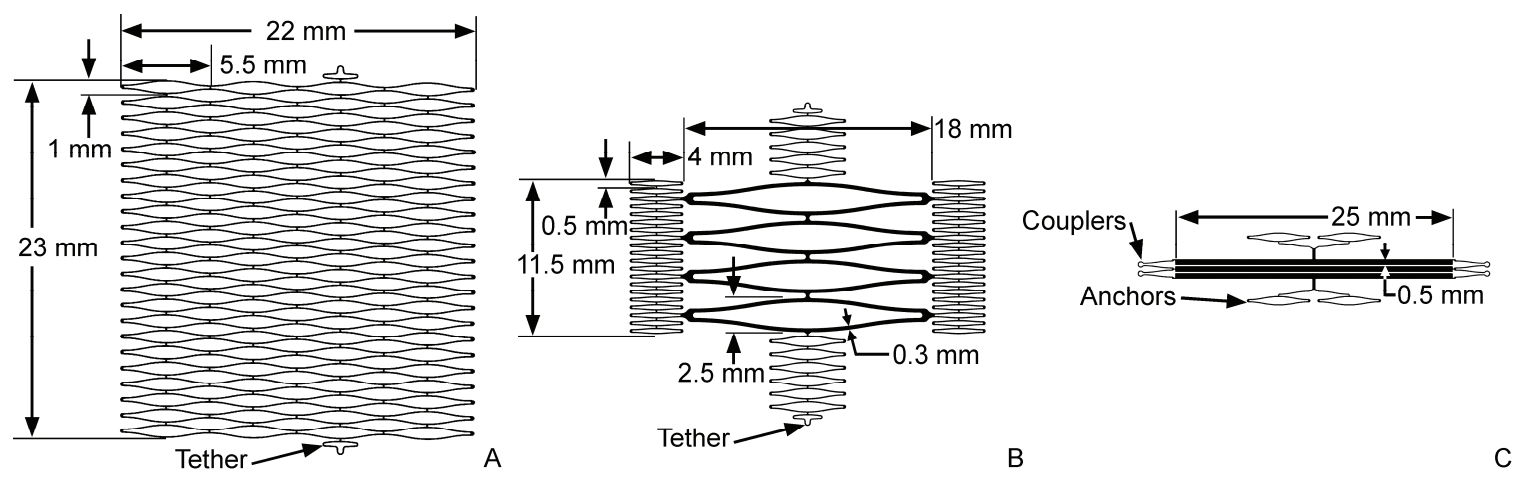

Fig. 5-1: Sensor design layout and important dimensions. A) Spatially sensitive design. B) Varying feature density design. C) Hybrid ribbon design. 
Three specific sensor designs are investigated here (Fig. 5-1). The first sensor design uses a pattern that resonates at two distinct frequencies, with each resonant frequency corresponding to a distinct modal shape. Because the modal shapes are distinct, each is affected differently by the spatial distribution of loads due to sludge accumulation; this allows monitoring of different locations along the length of the stent. The doctor can potentially use this added spatial information to aid in targeted therapies. The second sensor design implements a pattern with tailored feature density. The increased feature density at specific locations of the sensor results in a localized surface area increase. The increase in surface area has two effects at these locations: first, the sensor-to-environment coupling is improved; second, the biological components that contribute to sludge accumulation (proteins and bacteria) will tend to congregate in such regions [Coe90, Dow91, Dow92]. By tailoring the sensor geometry such that these regions of large surface area are located at antinodes of the resonant mode shape, sensor sensitivity is improved. The third sensor design uses three narrow ribbons that are coupled at the ends with flexible struts. The flexible struts allow the ribbons to slide over one another when the components are compressed inside the delivery sleeve. This design combines the relatively large signal amplitude and robust longitudinal mode shapes of simple ribbon sensors with the flexibility and expandability of the structures used in the first two designs.

This chapter also investigates an alternative magnetoelastic sensor material, as well as discusses thermal treatment for enhanced utility in this application. Detailed design considerations for the sensor topologies are first described, followed by fabrication information. Finally, experimental methods and results are presented and discussed. 


\subsection{Design}

\subsubsection{Material Selection}

The work described in previous chapters utilized Metglas ${ }^{\mathrm{TM}} 2826 \mathrm{MB}$ (a NiFeMoB alloy) as a material for the magnetoelastic sensors. According to the manufacturers, this alloy is specially developed for use in sensors. As described in this work (Chapter 2), ribbon sensors made from this material possess the highest quality factor and unloaded signal amplitude of the all the amorphous alloys investigated. However, as described in section 2.2.2.1, the damping added by the environment in which the sensor will be placed is high, and as such there are advantages to using a sensor material with a lower inherent quality factor. Namely, a sensor with impedance that is matched to the expected damping loads should maintain a high signal amplitude in the presence of those loads, while a sensor with mismatched impedance will have its signal amplitude severely attenuated. This point prompts the investigation of alternatives to the $2826 \mathrm{MB}$ alloy for use as the sensor material.

Of course, if alternative materials have a much lower unloaded signal amplitude than $2826 \mathrm{MB}$, then even an attenuated signal from a $2826 \mathrm{MB}$ sensor may be higher than the signal from an alternative material. For example, in Figure 2-9 (section 2.2.2.1), a 2 mm x $37.5 \mathrm{~mm}$ sensor of as-cast $2826 \mathrm{MB}$ retains a higher signal amplitude than an otherwise identical sensor of as-cast 2605SA1 for viscosities lower than about $3 \mathrm{cP}$, even though the signal amplitude of the 2605SA1 is less affected by increases in viscosity. Thus, a balance must be struck between amplitude and quality factor to ensure that the largest signal amplitude under loaded conditions is obtained. 
The balance between signal amplitude and quality factor is further affected by thermal treatments of the sensor material, as described in section 2.2.1.1. By annealing the sensor in a transverse magnetic field, the response of the sensor (as well as the required biasing field) can be significantly affected. The preliminary test described in section 2.2.1.1 (with $2 \mathrm{~mm} \times 37.5 \mathrm{~mm}$ ribbon sensors) shows that after appropriate thermal treatment, the signal amplitudes of the treated 2605SA1 and 2605CO sensors can be nearly as large as the treated 2826MB sensors. Additionally, the treated 2605SA1 and 2605CO sensors retain impedances that favorably match the expected loads. These points indicate that sensors made from treated 2605SA1 or $2605 \mathrm{CO}$ materials will perform more favorably in the intended application that sensors made from treated or ascast $2826 \mathrm{MB}$. As a final point to discriminate between treated 2605SA1 and treated $2605 \mathrm{CO}$, the required biasing field for the treated $2605 \mathrm{CO}$ sensors is $3.25 \mathrm{x}$ higher than the required biasing field for treated 2605SA1 sensors. Because higher biasing fields are more difficult to achieve (e.g. require thicker conformal magnetic layers), treated 2605SA1 presents the most favorable performance of the materials investigated in this work.

Discriminating between the as-cast and transversely annealed Metglas ${ }^{\mathrm{TM}}$ materials in light of the above analysis and the requirements of this application, this chapter focuses on transversely-annealed sensors fashioned from Metglas ${ }^{\mathrm{TM}} 2605 \mathrm{SA} 1$. The preliminary tests with ribbon sensors of this material show the best combination of large unloaded signal amplitude, structural damping that best matches the expected damping of viscous bile and viscoelastic sludge, and a low required biasing field. The results in the 
remainder of this chapter will illustrate the performance of the treated material in more complex sensor geometries.

\subsubsection{Geometry}

As illustrated in Fig. 5-1, three specific sensor geometries are given focus in this work. The intent behind each of these geometries is to preserve the advantages of the wishbone-array sensor presented in Chapter 4 - namely an open structure to provide mechanical expandability and flexibility - while improving functionality and performance by intelligent design of resonant mode shapes. This section describes the general concepts for each sensor design, points out specific features that provide the desired functionality, and presents predictions for the unloaded frequency response of each design.

The sensor geometries presented in this work are more complex than ribbon sensors, so the magnetomechanically-coupled finite element analysis (FEA) tool presented in section 2.2.3 is used to estimate frequency responses, mode shapes and expected signal amplitudes of the sensors. The material properties used in the model are based on available literature values, modified slightly to improve the fit with experimental frequency responses obtained from ribbon sensors fabricated from the actual material used. The predicted responses are compared with experimentally measured responses for each sensor in Fig. 5-2. Note that the match between the FEA predicted response and the experimentally measured response becomes worse as the main features of the vibrating pattern become smaller. The material parameters used in the FEA model were established by fitting the measured response of transverse-field-annealed ribbon sensors - 
one $2 \mathrm{~mm} \times 30 \mathrm{~mm}$ and one $1 \mathrm{~mm} \times 15 \mathrm{~mm}$ - that had been photochemically machined from a planar foil of 2605SA1. The fact that the match between FEA predicted performance and measured performance for these more complex geometries worsens with changes in feature size indicates that feature size may have a significant effect on transverse field annealing as well as critical material properties. This issue is discussed further in Chapter 7.
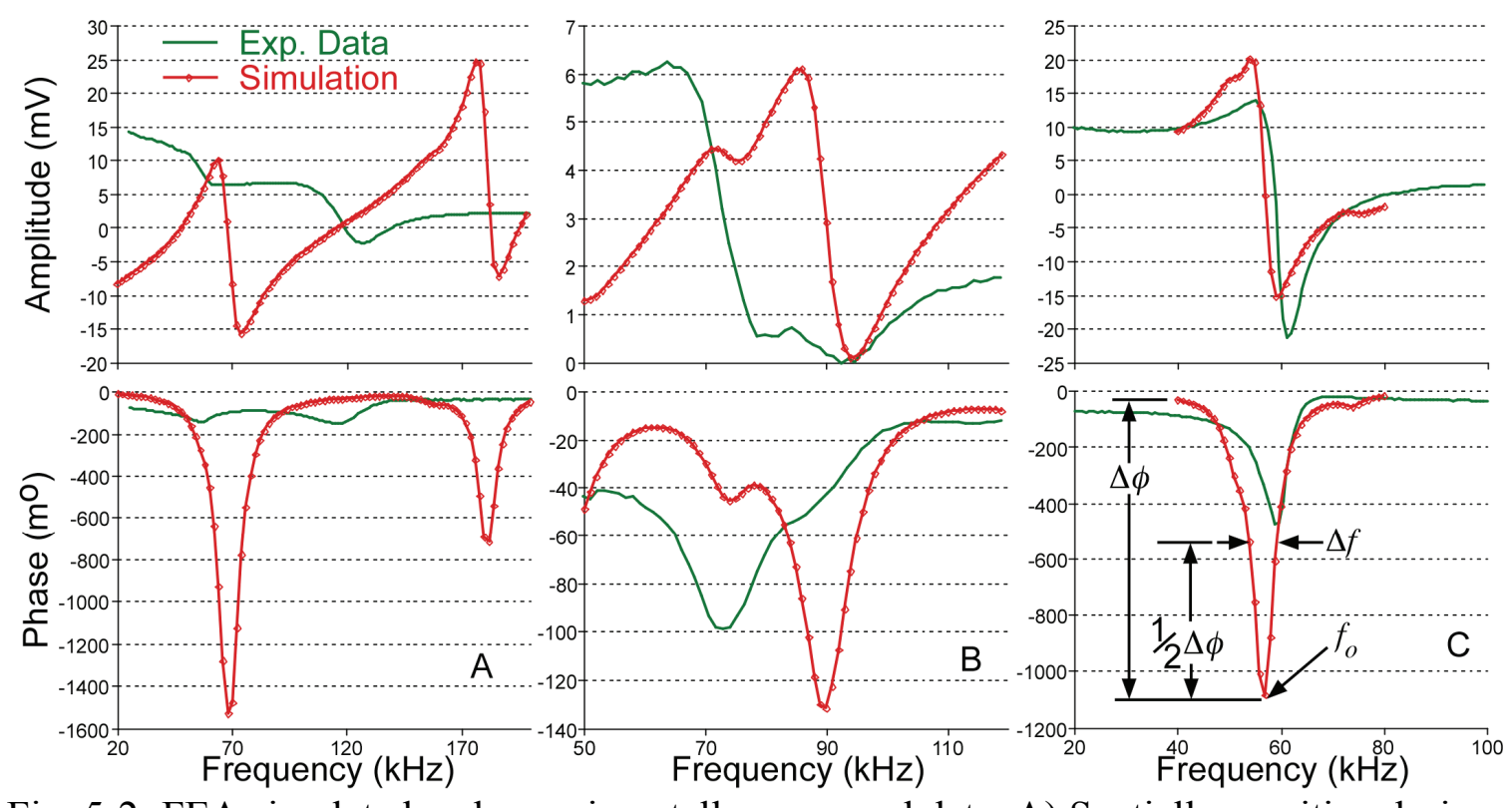

Fig. 5-2: FEA simulated and experimentally measured data. A) Spatially sensitive design. Note that the sensor produces two well-separated resonant responses. B) Varying feature density design. C) Hybrid ribbon design. In the phase chart, the half-power bandwidth and the characteristic frequency of the phase dip are labeled. These parameters are used throughout the results presented in this chapter.

\subsubsection{Spatially Sensitive Design}

The first sensor design uses a wishbone-array pattern that is similar to the pattern used in sensors presented previously. However, the dimensions of each cell in the pattern and the overall active area of the sensor (Fig. 5-1A) have been modified to provide two 
advantages. First, the cell has been elongated to result in more expandability. Second, the elongated cell and the wider yet shorter active area results in a tailored frequency response that allows for spatially sensitive monitoring. The sensor resonates at two distinct frequencies (near $50 \mathrm{kHz}$ and $110 \mathrm{kHz}$ when unloaded, although predicted at 64 $\mathrm{kHz}$ and $177 \mathrm{kHz}$ respectively), with each resonant frequency corresponding to a distinct mode shape (as shown in Fig. 5-3). The first mode shape - corresponding to the lower resonant frequency - is mainly longitudinal in nature, and produces longitudinal movement of the ends of the active area of the sensor. Meanwhile, the cells in the midlength of the active area open and close in a transverse motion. In contrast, the second mode shape - corresponding to the higher frequency - shows relatively little movement of the sensor ends, while still showing transverse movement in the cells near the midlength of the sensor. The spatial arrangement of the nodal and anti-nodal regions of each mode shape determines the sensitivity of the frequency response (i.e. resonant frequency, characteristic damping) of each mode shape to the spatial distribution of sludge. For instance, the presence of sludge at the ends of the active area will act mainly as a mass load on the anti-nodal regions of the first mode shape while acting as a slight stiffening and damping load on nodal regions of the second mode shape. Thus, the lower resonant frequency will shift down, while the higher resonant frequency will shift up slightly (due to increased effective stiffness) or will exhibit a lower quality factor (due to increased damping). Alternatively, the presence of sludge at the middle of the active area will lead to an increase in the first mode resonant frequency (due to increased effective stiffness) or a decrease in quality factor (due to increased damping). The presence of sludge at the middle of the active area is expected to have a more complex effect on the frequency 
response characteristics, depending on the nature of and distribution of the load. For instance, sludge that has not bridged the interstices of the wishbone-array cells will act primarily as a mass load, lowering the resonant frequency of the mode shape. In contrast, sludge that has bridged the interstices of the wishbone cells may significantly stiffen the structure or add significant damping, resulting in an increase in resonant frequency or a decrease in quality factor, respectively.

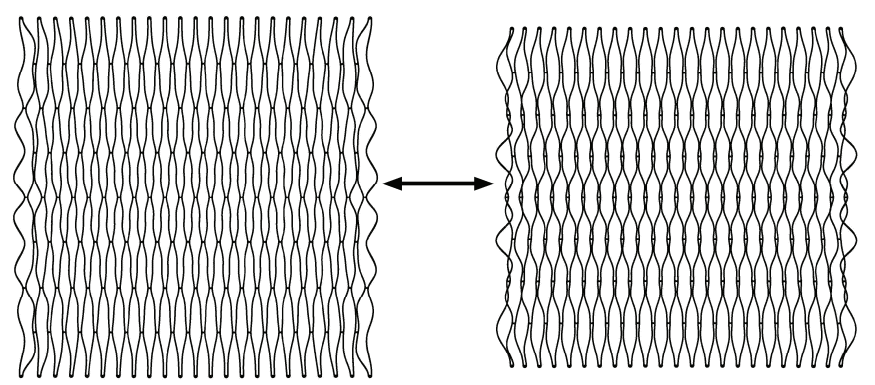

$64 \mathrm{kHz}$

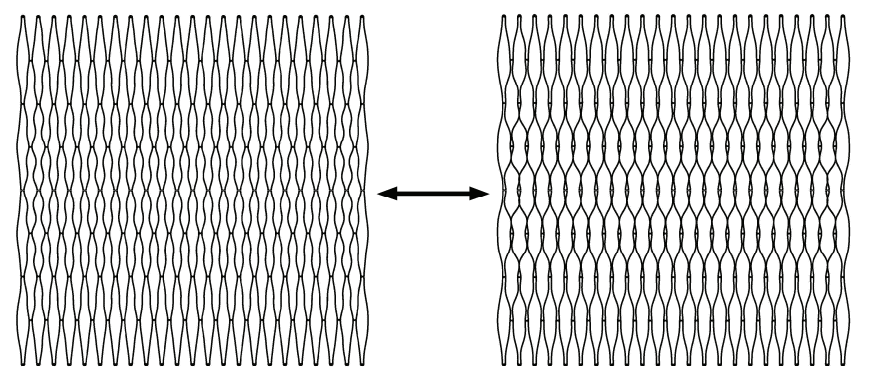

$177 \mathrm{kHz}$

Fig. 5-3: FEA simulated mode shapes for the spatially sensitive design, along with predicted resonant frequencies. The first mode shape (top) produces extension and contraction of the sensor along the longitudinal direction, while the second mode shape (bottom) results in little length change but large transverse movement of the struts at the middle of the sensor.

Based on the dimensions of the pattern and the mode shapes of each frequency, this sensor should be able to resolve the location of sludge accumulation within about $5 \mathrm{~mm}$ along the length of the stent. This added functionality can lead to two benefits for the monitoring physician. First, the effects of non-uniform accumulation of sludge will no 
longer be averaged over the entire active area. This gives the physician better information about the local severity of the disease. Secondly, the doctor can better direct any therapeutic efforts that require such spatial information - e.g. ultrasonic lithotripsy or targeted drug delivery.

\subsubsection{Varying Feature Density Design}

The wishbone-array sensor presented in Chapter 4 and the first sensor design in this chapter use a repeated cell throughout the active area. In contrast, the second sensor design implements a pattern with varying cell size. The varying feature density design is intended to illustrate one method for improving the sensitivity of the sensor by causing sludge producing bacteria to selectively congregate at predetermined locations that are most sensitive to loading - the antinodes of the resonant mode shape - rather than congregating uniformly across the sensor. As shown in Fig. 5-1B, larger cells are used in the middle of the active area, while much smaller cells are used at the ends of the active area. This design results in a resonant mode shape that uses the larger features in the middle of the sensor to drive the movement of the smaller features at the ends of the sensor. Thus, the antinodal locations are specifically given a large effective surface area. In this specific application, an increase in surface area and the presence of fine features can also lead to another favorable result: according to several studies, the biological components that contribute to sludge accumulation (proteins and bacteria) will tend to congregate in such regions [Dow91, Dow92, Coe90]. In these studies, sludge accumulation was significantly increased around the irregular rims of side holes and near irregular defects on the inner surface of plastic stents, accompanied by an aggregation of 
bacteria. The authors hypothesize that the increased surface area due to these irregularities plays a large part in the behavior of the bacteria. In general applications,

the increased surface area - especially of surfaces that are normal to the direction of oscillation - will improve sensor-to-environment coupling through the generation of normal waves. Normal waves are a contrast to the shear waves that are generated from the sensor surfaces that are parallel to the direction of oscillation; the effect of shear waves tends to decay quickly in materials that do not support shear stress well, including liquids (such as bile) and biofilms with relatively low rigidity (such as sludge).

\subsubsection{Hybrid ribbon sensor}

The third sensor design detailed in this paper represents a hybridization of the ribbon and wishbone-array sensor types studied in our previous work in an effort to combine the respective advantages while circumventing the respective disadvantages of each sensor type. As shown in Fig. 5-1C, the hybrid ribbon design uses three parallel narrow ribbons that are connected at the ends with flexible linkages. The flexible linkages allow the ribbons to slide over one another when the components (stent and sensor) are compressed inside the delivery sleeve; upon removal of the delivery sleeve, the linkages elastically return to their fabricated shape and hold the ribbons apart. Because the linkages are located at the antinodes of the individual ribbons, they also serve to couple the vibrations of the ribbons and enhance the response of the sensor: analytical and experimental results show that the signal amplitude from three coupled ribbons is three times larger than that from a single ribbon. The linkages thus provide the hybrid ribbon sensor with the advantages that are typical of wishbone-array sensors - namely, maintaining 
expandability while allowing an increase in the amount of magnetoelastic material (and associated improvements in signal strength). The ribbons in the hybrid sensor vibrate in a simple bulk longitudinal mode. This mode shape is advantageous for this application relative to the complicated modes excited in a wishbone-array sensor for two main reasons. First, the longitudinal mode shape is coupled into much more strongly by the transmitted magnetic field, which is directed primarily longitudinally. Second, the longitudinal mode shape is more robust against the mass, stiffness, and damping loads presented by the accumulation of sludge in the interstices of the sensor. In contrast, the bending that takes place in the struts in the mode shapes of a wishbone-array sensor is heavily affected by any interstitial material. Thus, the design of the hybrid ribbon sensor combines the favorable mode shapes of ribbon sensors with the desirable expandability of the wishbone-array sensors.

\subsection{Fabrication}

The sensors are batch-patterned from a $25 \mu \mathrm{m}$ thick foil of as-cast 2605SA1 Metglas $^{\mathrm{TM}}$ utilizing the PCM process (see section 3.1.1). Feature sizes of the individual struts are $100 \mu \mathrm{m}$. Other important dimensions are shown in Fig. 5-1.

To improve the signal amplitude, the sensors are thermally treated in a large transverse magnetic field. Sensors are layered in between glass slides that are held together with an Elgiloy ${ }^{\mathrm{TM}}(\mathrm{Cr}-\mathrm{Ni}$, non-magnetic) retaining spring. This stack is then placed in a $\sim 1.5 \mathrm{kOe}$ magnetic field provided in the 1.5 inch wide gap of an AlNiCo major magnet, with the field directed transverse to the long axis of the sensor. The stack 
was then heated above the Curie temperature for $2605 \mathrm{SA} 1\left(395^{\circ} \mathrm{C}\right)$ and held at temperature $\left(413^{\circ} \mathrm{C}\right)$. After cooling, the stack was removed and the sensors individually tested for signal improvement. It was found that the signal continued to improve with time held at temperature, at least up to 3 hours. With a 3 hour treatment, the signal amplitude for the spatially sensitive design improved by an average of $5 x$, the signal amplitude for the varying feature density design improved by an average of $4 x$, and the signal amplitude for the hybrid ribbon design improved by an average of $15 x$. Note that the average amplitude improvement as a result of the transverse field annealing process is dependent on sensor design. This dependency may be a result of feature size or feature density affecting the ability of the transverse field to penetrate the sensor material, or it may be a result of the more transverse mode shapes in the spatially sensitive and varying feature density designs being less improved by the reorientation of magnetic domains.

It was found that the number of sensors in the stack did not significantly affect the treatment outcome, as long as the sensors remained in between the poles of the magnet. Additionally, the permeability of the material used to sandwich the sensors had little effect on the outcome. These two observations indicate that a large batch of sensors can be transversely annealed simultaneously between thin stacking layers.

The stent application calls for a generally tubular shape, so the sensor must be curved into a tubular or semi-tubular shape to best match the stent geometry. Initial attempts to add curvature to the sensor via elastic bending (e.g. by rolling the sensor and stent into a tube) resulted in a resonant frequency shift and a severe decrease in the amplitude of the sensor signal. This effect is thought to be the result of a combination of mechanical stress imposed in the material, as well as geometrical changes in the mode shapes. The 
tubular shape is achieved in this work by placing the sensor against the inner wall of a metal tube and annealing at $375^{\circ} \mathrm{C}$. By annealing the sensor in the curved state, mechanical stress in the material is relieved while the desired shape is maintained, leaving only geometrical changes in the mode shapes to affect the sensor response. These changes are less significant than those imposed by mechanical stress, especially if design steps are taken anticipating the curvature (e.g. FEA models that incorporate curved designs). This thermal treatment should be applied to the sensor below the Curie temperature of the material in order to retain the signal amplitude gain resulting from the previous treatment in the transverse magnetic field. The amount of springback that occurs after this treatment varies for each sensor design as a result of the different bending stiffness of each design. Therefore, the diameter of the metal tube and the annealing time must be chosen for each design to achieve the desired final curvature. As an example, a 6-hour treatment in a $5 \mathrm{~mm}$ diameter tube results in a $9 \mathrm{~mm}$ final curved diameter for the spatially sensitive and varying feature density designs. For the hybrid ribbon design, a 1.5 -hour treatment in a $2.3 \mathrm{~mm}$ diameter tube results in a $7 \mathrm{~mm}$ final curved diameter.

The iron content in the amorphous alloys can result in a tendency for the material to corrode in aqueous environments, shifting and diminishing the sensor response. To passivate the material, the sensors are coated in a conformal layer of $2 \mu \mathrm{m}$ thick Parylene-C (polyxylylene) using a standard vacuum deposition technique. This process results in minimal shifts in the frequency response and amplitude of the sensors. The coating process should be done subsequent to the thermal processes described above, as the polymer coating is susceptible to degradation at the high temperatures involved in 
those processes. After this process, the sensors are notably more robust in corrosive environments, with no coated sensors failing due to corrosion through the duration of the testing carried out here. However, further tests should be done to ensure the long-term stability and biocompatibility of the coated sensors. Figure 5-4 shows the measured signal from a hybrid ribbon sensor after passing through each of the process steps described above: photochemical machining, transverse field annealing, thermal curvature addition, and parylene coating. The shifts in amplitude and frequency response for each step are similar for the other two designs (although the magnitude of the amplitude change after transverse field annealing is for the other designs is smaller, as noted previously).
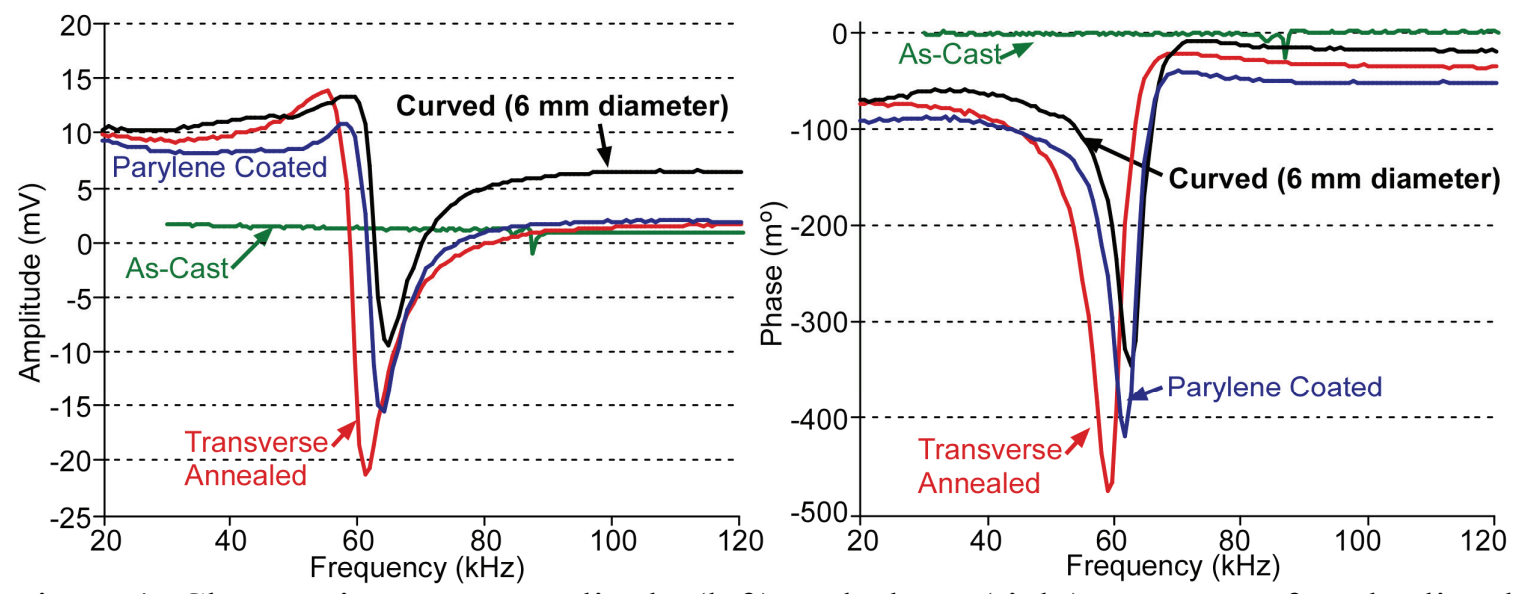

Fig. 5-4: Changes in sensor amplitude (left) and phase (right) response after the listed treatments applied to the hybrid ribbon design. Note that the transverse field annealing step produces a huge change in response amplitude and phase. Changes due to parylene coating and addition of curvature are similar in magnitude and much smaller than the changes due to transverse field annealing.

Lateral portions are included in each sensor design to facilitate mounting of the sensor to the inner sidewall of the stent. In the spatially sensitive and varying feature 
density designs, tethers (as shown in Fig. 5-1A,B) can be woven into the stent structure using any of the braided wires that make up the most commonly used commercial stent design (Boston Scientific Wallstent ${ }^{\mathrm{TM}}$ ). Alternatively, with stent designs that are patterned from a single piece of metal, integrated mounting wires can be included in the stent pattern for weaving into the sensor tethers. In the hybrid ribbon design, anchors are connected to the active sensor area with struts (see Fig. 5-1C). The anchors can be bonded to the stent with a number of methods: a thin layer of PDMS (as used in this work) or other biocompatible adhesive, with a parylene-parylene bond [KimH05], or with welding. Note that either of the mounting features described here (i.e. tethers or anchors) are interchangeable among the sensor designs. For this work, the mounting processes were verified experimentally to have no significant effect on the sensor response. Examples of spatially sensitive and hybrid ribbon designs integrated with a commercial stent that has been coated with a conformal magnetic biasing layer are shown in Fig. 5-5.

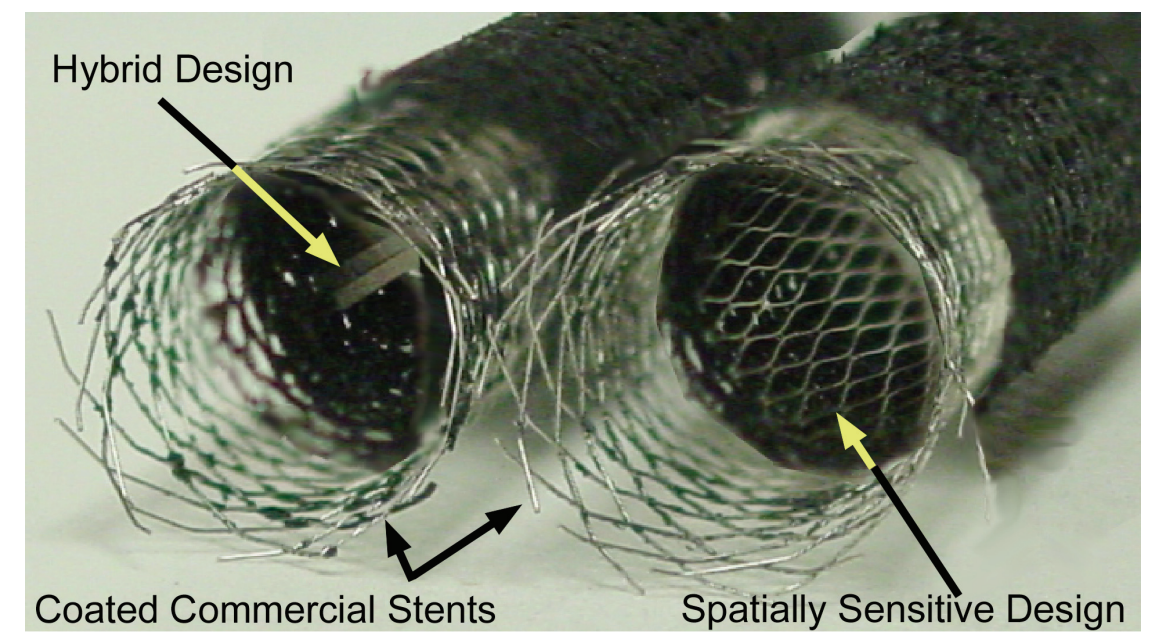

Fig. 5-5: Sensors integrated with commercial self-expanding biliary stents $(8 \mathrm{~mm} \times 60$ $\mathrm{mm}$, Boston Scientific Wallstent ${ }^{\mathrm{TM}}$ ). The sensors anchors are bonded to the stent with PDMS. 


\subsection{Experimental Methods}

Isolated sensors (i.e. not attached to stents) are tested unless otherwise noted. Magnetic biasing of the sensors is applied through the use of dual Helmholtz coils. The coaxial configuration (A) is used for interrogation of the sensors for all test results reported here.

Paraffin is once again used as a sludge simulant. For the varying feature density and hybrid ribbon designs, the entire sensor is immersed in the melted paraffin and then removed to build up successive layers. For the spatially sensitive design, this process is modified slightly to better control the spatial distribution of the paraffin so that effects on each mode shape can be separately characterized. The paraffin is added to the ends of the spatially sensitive sensor by dipping only the ends of the sensor into the melted paraffin, one end at a time. To add paraffin to the middle of the sensor, melted paraffin is placed in a pipette and drizzled onto the desired location before solidification occurs.

Before and after adding mass to the sensors, measurements are taken with the sensors immersed in fluids of various known viscosities (air; DI water, Dow Corning 200 2, 5, 10 , and $20 \mathrm{cS}$ fluids). This procedure allows for further experimental investigation into the separation of mass and viscosity effects, a procedure described in section 2.2.2.2. It was found that immersion in the Dow Corning fluids tended to change properties of the paraffin layers, so measurements after immersion in a Dow Corning fluid required that the sensor be cleaned and paraffin reapplied before further testing. This requirement hurts the repeatability of the test, as each paraffin coating would result in a slightly different distribution on the sensor. The results presented still provide good insight into the device behavior, but in the future more rigorous characterization should be done with 
fluids that do not affect the sludge simulant.

To investigate the effect of sludge material properties on the response of the sensor, an alternative method for simulating sludge is also used. With this method, the sensors are first separately positioned inside $6-8 \mathrm{~mm}$ diameter plastic tubes (the diameter of the tube used matched the diameter of the curved sensor). Subsequently, the sensors and tubes are immersed in a gelatin-water mixture (1/2 oz of unflavored gelatin powder fully dissolved in $144 \mathrm{~mL}$ of deionized water). The mixture is allowed to fully cure (via refrigeration for 6 hours), and then the sensors and tubes are removed. At this point, the tubes are completely filled with cured gelatin, and the sensor is completely encased along the sidewall of the tube. After allowing the gelatin to reach room temperature $(\sim 15$ minutes), the response of the sensor is measured. Then, a thin-walled metal tube is used to core out a channel in the gelatin, near the center axis of the tube. Again, the sensor response is measured. Subsequently, larger diameter channels are cored out of the gelatin followed by measurement of the sensor response. This procedure allows the response of the sensor to be correlated to a patent diameter, with the remaining gelatin representing the occluding sludge. For this portion of the testing, the sensor (and tube and gelatin) is not immersed in liquids because of the concern that liquids might change the gelatin properties and result in a poorly controlled test.

\subsection{Experimental Results}

Figure 5-6 shows the results for paraffin loading of the spatially sensitive design. As can be seen, each of the characteristic frequencies responds differently to the presence of 
paraffin in the two locations (ends of the sensor, middle of the sensor). Note that the characteristic frequency of the second mode tends to increase when paraffin is placed on either location, albeit with a different sensitivity for the two locations. This indicates that the paraffin tends to add stiffness to the vibration of the second mode shape, likely by bridging the interstices of the pattern. This trend is also seen in the characteristic frequency of the first mode when mass is added to the middle of the pattern.

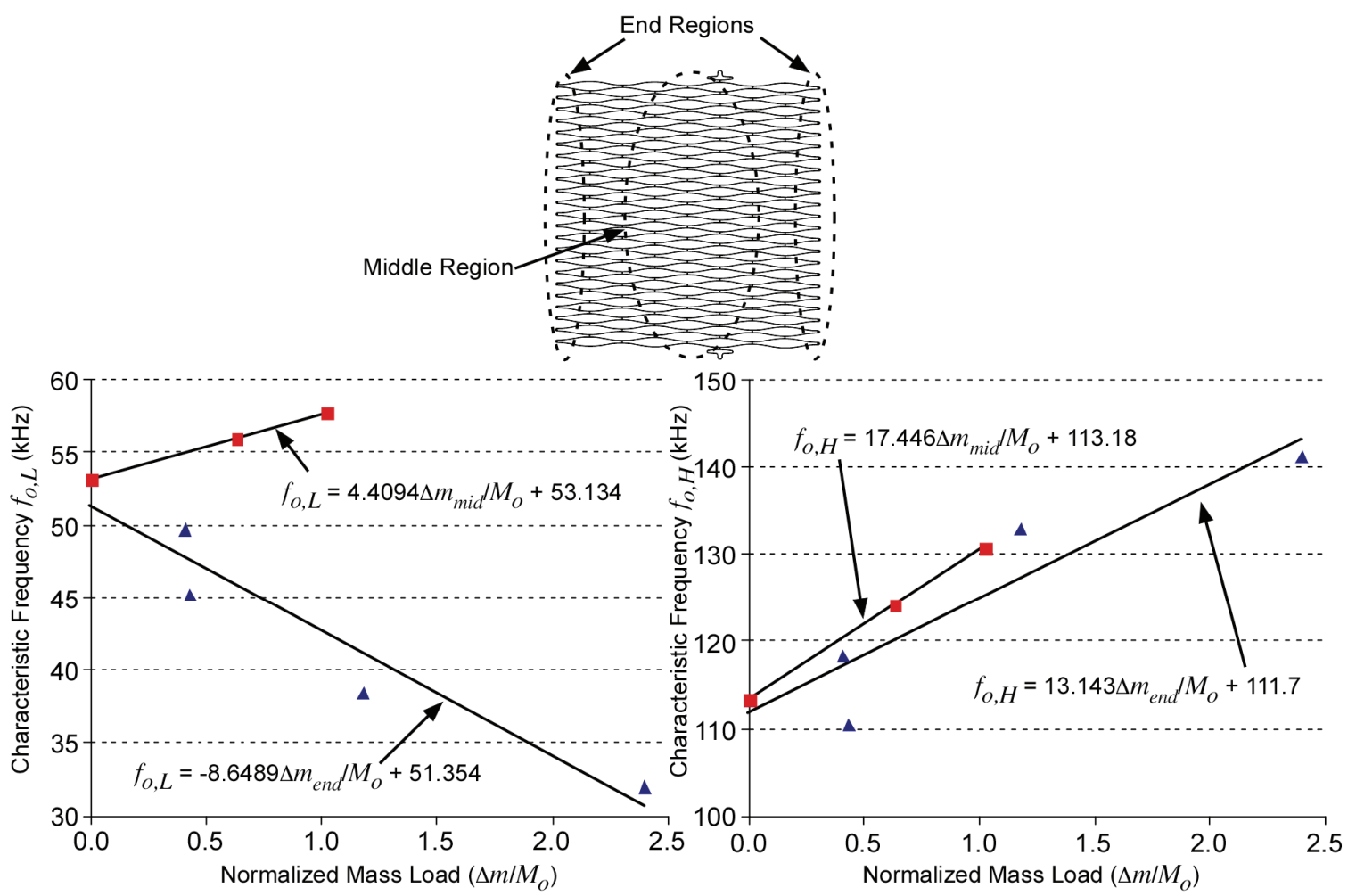

Fig. 5-6: Paraffin loading results for the spatially sensitive sensor. The top sketch illustrates the locations where paraffin is applied for the "mid" and "end" responses. The left chart shows that the characteristic frequency of the first mode decreases as mass is added to the end, while the characteristic frequency increases as mass is added to the mid-length. The right chart shows that the characteristic frequency of the second mode increases for mass added at both locations, with a slightly different rate for each location.

The response of the two characteristic frequencies can be used to separately characterize the amount of paraffin in each location using only the measured 
characteristic frequencies. First, the equations for each characteristic frequency in Fig. 56 are superposed, then $\Delta m_{e n d} / M_{o}$ and $\Delta m_{m i d} / M_{o}$ are solved for in terms of $f_{o, L}$ and $f_{o, H}$. The resulting relationships can be used to estimate the amount of mass at each portion of the stent, as shown in Table 5-1. Further characterization and empirical modeling may improve the ability to estimate the distribution of the mass loads.

Table 5-1: Calculated mass distribution using measured characteristic frequencies

\begin{tabular}{|c|c|c|c|c|c|}
\cline { 3 - 6 } \multicolumn{2}{c|}{} & \multicolumn{2}{c|}{ Measured } & \multicolumn{2}{c|}{ Calculated } \\
\hline$f_{o, L}(\mathrm{kHz})$ & $f_{o, H}(\mathrm{kHz})$ & $\Delta m_{\text {end }} / M_{o}$ & $\Delta m_{\text {mid }} / M_{o}$ & $\Delta m_{\text {end }} / M_{o}$ & $\Delta m_{\text {mid }} / M_{o}$ \\
\hline 50.88 & 125.5 & 0.4046 & 0.3817 & 0.3929 & 0.4605 \\
\hline 45.25 & 130.75 & 1.2366 & 0.3817 & 0.9732 & 0.3219 \\
\hline 47.5 & 128.5 & 0.4275 & 0.5115 & 0.7381 & 0.3709 \\
\hline
\end{tabular}

Figure 5-7 shows the results for paraffin loading of the varying feature density design, along with a curve representing a typical response for the sensor designs described in Chapters 3 and 4 . Note that the resonant frequency of the sensor is much more sensitive than previous designs to the addition of mass throughout this range. Additionally, it was observed that, due to meniscus formation across the small features at the ends of the sensor, the paraffin tended to build up in the areas of high feature density (as expected for sludge).

Figure 5-8 shows the separation of viscosity and mass effects for the varying feature density design using the procedure described in section 2.2.2.2 (i.e. using equation (20) and fitting parameters $\mathrm{C}$ and $\mathrm{D}$ to the data). Although the data is not fit precisely, general trends are fit well. Further data collection and empirical model fitting may improve the precision of this method. 


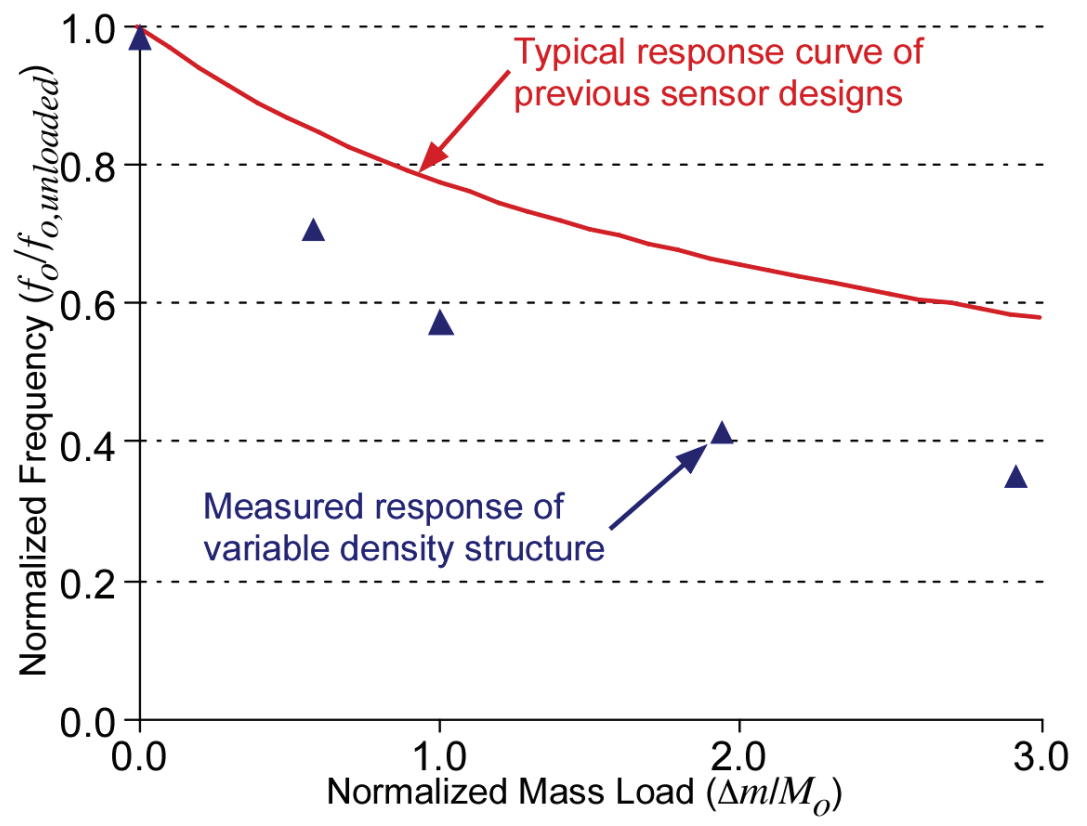

Fig. 5-7: Paraffin loading results for the varying feature density design. Note the increased sensitivity as compared to designs presented in Chapters 3 and 4.

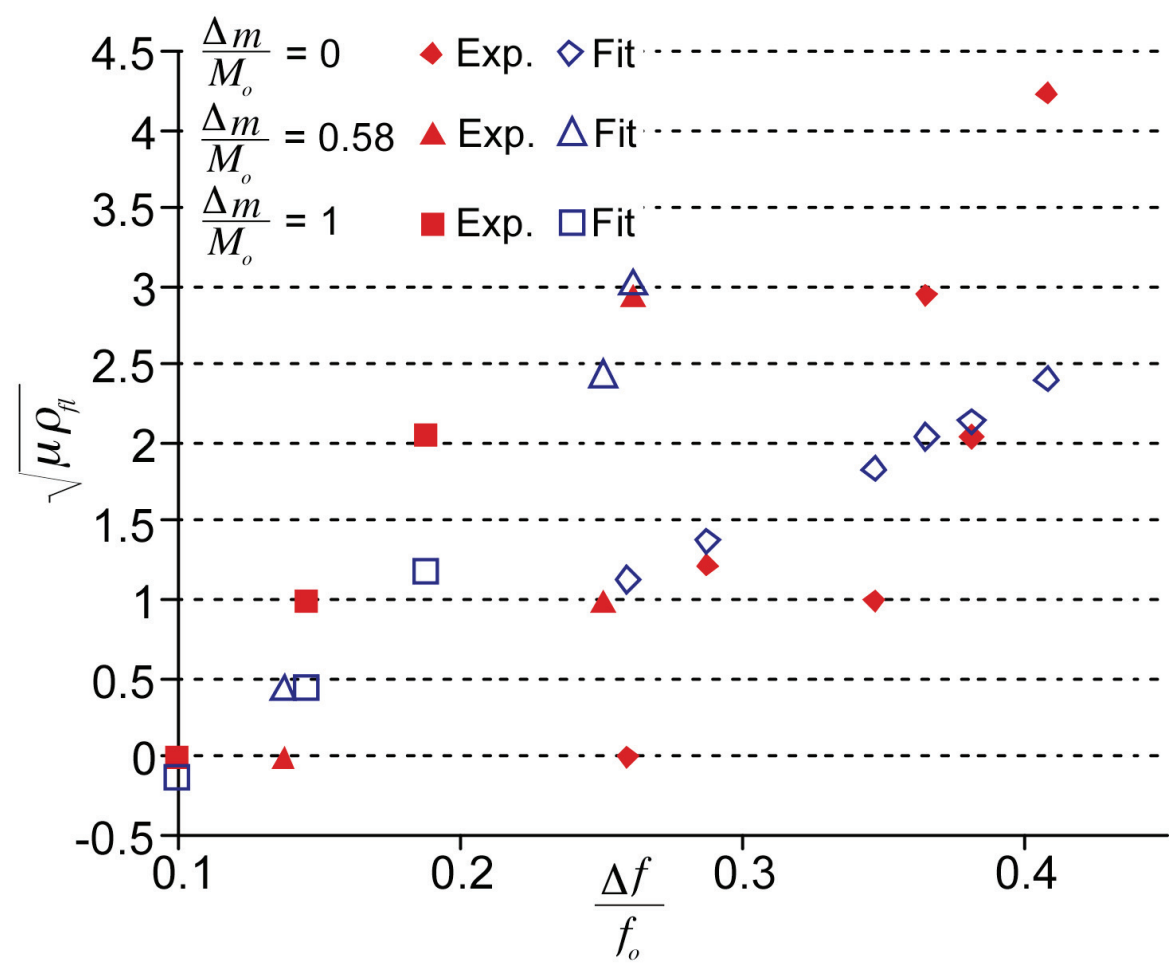

Fig. 5-8: Separation of effects of viscosity from those of paraffin mass loads for the varying feature density design. Although the fitted values are not exact, the trends are well predicted. 
Figure 5-9 shows the results for paraffin loading of the hybrid ribbon design. The hybrid ribbon shows slightly worse sensitivity to paraffin loading than ribbon sensor designs discussed in Chapters 3 and 4; this may be due to the very different aspect ratio of the ribbons used in the hybrid ribbon design compared to previous designs, or it may be different because the paraffin tended to be distributed differently on the sensor due to the small gaps between the ribbons or due to other small features. However, the characteristic frequency response is still well fit with only a small modification of the relationship suggested by the analytical model for a simple ribbon sensor given in equation (19).

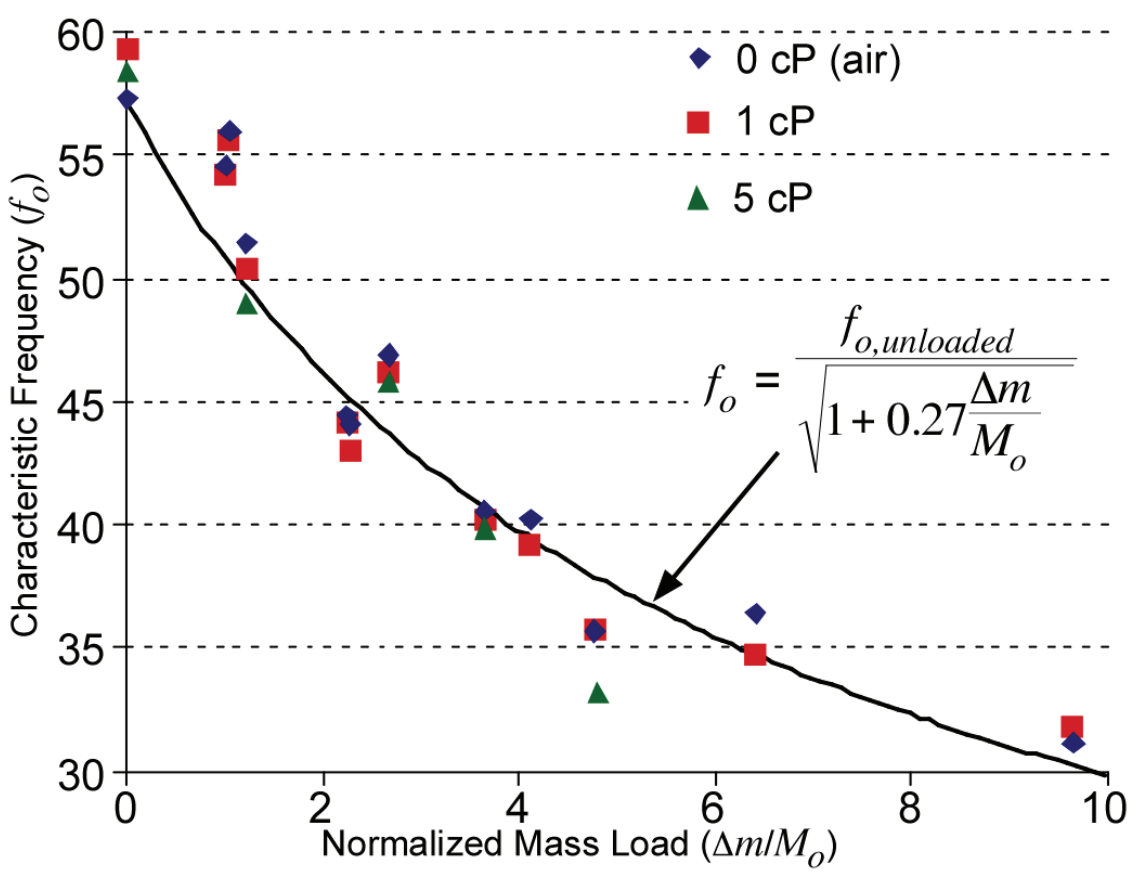

Fig. 5-9: Paraffin loading results for the hybrid ribbon design. Note the minimal effect on characteristic frequency that a change in viscosity produces. The curve is fit to the data points using an equation with the form shown and varying the term multiplying the normalized mass load. The variability in data for similar mass loads is likely attributable to a slightly different (and non-uniform) distribution of mass on the sensor for each mass load. 
Figure 5-10 shows the separation of viscosity and mass effects for the hybrid ribbon design. Again, the general trends for viscosity are fit well, and improvement may be possible through further data collection and parameter fitting. In general, the varying density is slightly more sensitive to shifts in viscosity than the hybrid ribbon sensor, with $\Delta f / f_{o}$ varying over a wider range as viscosity changes for a given normalized mass load. This larger sensitivity is again thought to be an effect of the greater interaction of the small features at the ends of the varying density design with the environment.

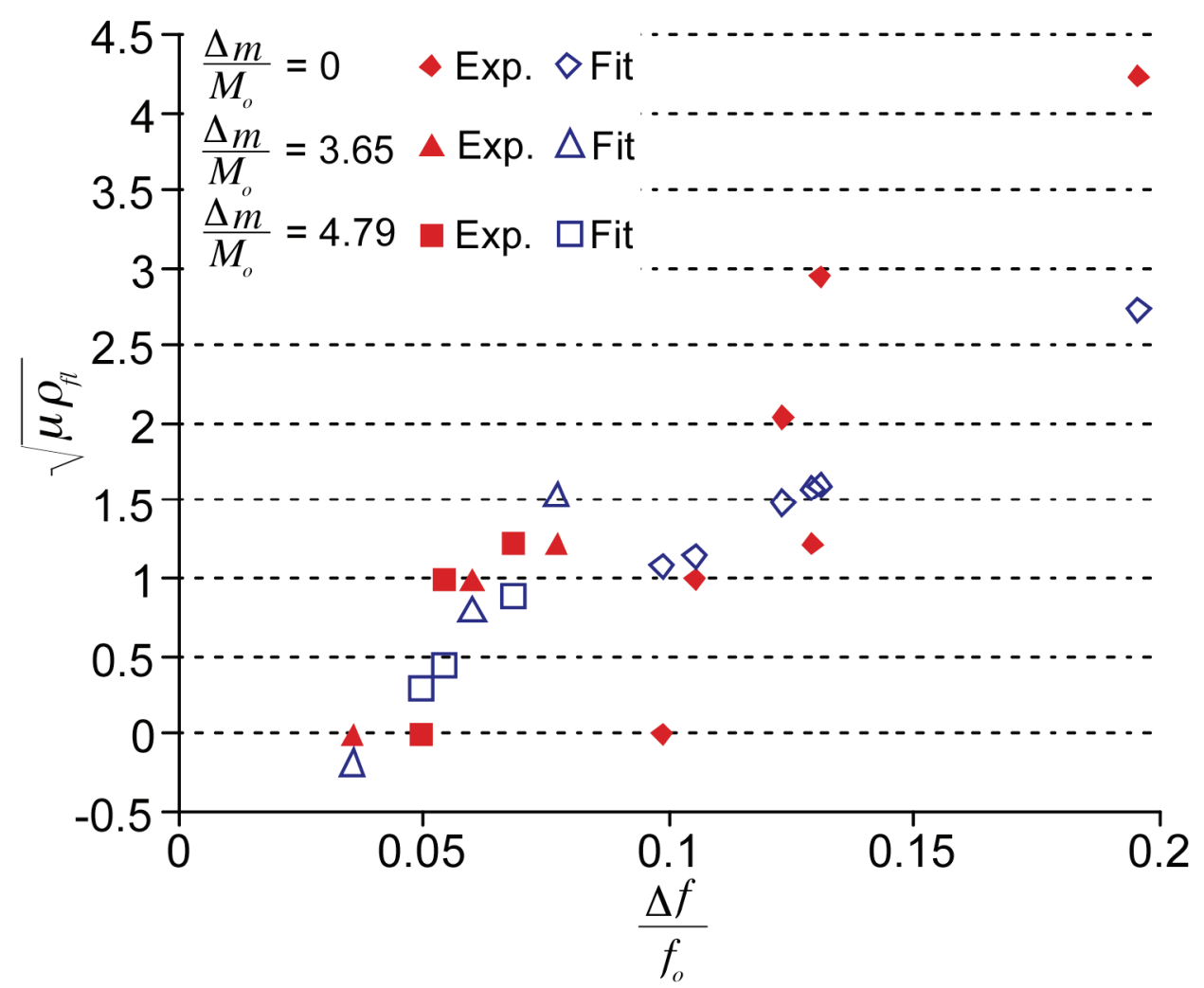

Fig. 5-10: Separation of effects of viscosity from those of paraffin mass loads for the hybrid ribbon design. The results for large mass loads and low viscosities are especially well fit.

Figure 5-11 shows the results for three trials using gelatin and a hybrid ribbon sensor (a $6 \mathrm{~mm}$ diameter tube was used for these trials). Note that, as the patent diameter 
increases, $\Delta f / f_{o}$ decreases. These results indicate that, within a given test run, the sensor can resolve the difference between $2 \mathrm{~mm}$ of patent diameter and a fully occluded duct, using $\Delta f / f_{o}$ as the measurand. The trends for the other designs are similar and indicate similar resolution within test runs.

When the sensor performance under gelatin loading is evaluated over different test runs, further insight is gained. Though the trend in the sensor response for each trial is similar, the variability between trials is large. For instance, a measured $\Delta f / f_{o}$ of 0.16 indicates a patent diameter of about $1 \mathrm{~mm}$ for one test run, while the same measured value indicates a patent diameter of about $4 \mathrm{~mm}$ for another test run using the same sensor in the same tube. Determining the source of this variability is an important part of fully evaluating the sensor performance. In this case, the variability from test to test may be due to a number of sources. One potential source of variability is the slightly different distribution of gelatin around the sensor after coring out a flow channel. Because the mode shape (and thus sensitivity to loads) is distributed spatially, any variation in the spatial distribution of the loads will result in a different sensor response. This variability is also seen with the paraffin testing, but at a much less significant level. Another potential source of variability is the gap between the sidewall and the sensor. Because the sensor is not fixed in the tube, a different gap between the sidewall of the tube and sensor may exist for each run. This can result in a different boundary condition for the sensor, which will affect the response. An additional source of variability may be the material properties of the gelatin due to processing variations. Unfortunately, in the end application all of these sources of variability are likely to exist: sludge will accumulate with a different distribution each time, sludge components (and hence material 
properties) will be different in each case, and the sensor may be deployed such that a slightly different gap between the inner duct wall and sensor exists. Future tests can be designed to reduce these potential sources of variability and evaluate which are the most important in determining the sensor response. Then, designs can be modified to reduce sensitivity to these sources of variability.

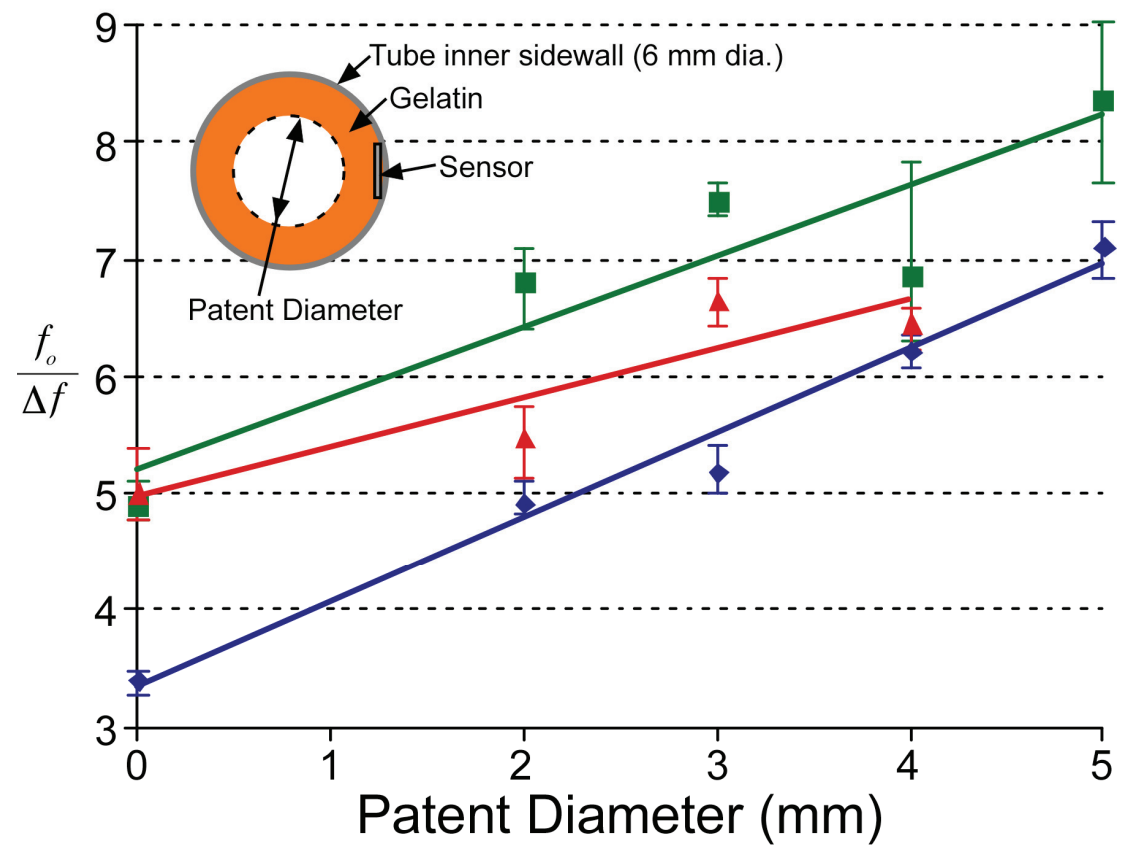

Fig. 5-11: Gelatin loading results for the hybrid ribbon design. Three separate runs using the same sensor are shown, fit with linear regression. Error bars for each data point signify the range of values obtained from three measurements at that point, while the data point signifies the average of the three measurements. Note that, within a test run, the sensor demonstrates a resolution of $\sim 2 \mathrm{~mm}$ of patent diameter, and remains sensitive until the tube is fully occluded. However, the absolute sensor output shows a large variability from one test run to the next.

In contrast to paraffin loading, the gelatin loading affects the quality factor of the resonance (as measured by $f_{o} / \Delta f$ ) more significantly than the characteristic frequency $\left(f_{o}\right)$. The results of this testing indicate that, for a viscoelastic load, the important parameter to measure depends on which regime (viscous or elastic) is more dominant for the loading 
material in question. For a load in which the elastic regime dominates (i.e. stiffer materials like paraffin), the characteristic frequency changes appreciably as mass increases. For a load in which the viscous regime dominates (i.e. less rigid materials like gelatin), the quality factor of the system changes appreciably as the load accumulates. Regardless of the dominant regime, these tests show that the magnetoelastic sensor can provide useful information about the state of the environment around the sensor.

\subsection{Discussion}

The work presented in this chapter explores some of the possibilities for enhanced sensor functionality via tailored device geometry. Along with an understanding of how the dynamics of the sensor structure are affected by environmental loads, the use of the design tools presented in this work and the application of the photochemical machining process can produce sensors with spatial sensing functionality, improved sensitivity, and robust mechanical properties. Many other variations on the ideas presented in this chapter can also be envisioned, each with potential performance enhancements, but the framework laid here in the form of devices and results is important.

The spatially sensitive design presented in this chapter is able to discriminate between accumulation of material at the ends of the sensor and accumulation of material toward the middle of the sensor, corresponding to a spatial resolution of about $5 \mathrm{~mm}$ along the length of the sensor. The basic concept of using mode shapes with sufficiently different frequency responses (so that each response can be measured separately) and targeted nodal and anti-nodal locations for spatial sensitivity can be applied to many different 
design architectures. The particular advantage of the open, mesh-like architecture studied here - expandability - is specific to the application in self-expanding biliary stents or in any other application where minimizing the sensor profile prior to deployment is key. In fact, spatially sensitive designs mounted in commercially available self-expanding stents survive compression into and redeployment from a $2 \mathrm{~mm}$ diameter delivery sleeve, with no significant changes to signal amplitude or frequency response.

As predicted, the varying feature density design shows a $\sim 2 \mathrm{x}$ sensitivity increase to both mass loading and viscosity shifts over previously described ribbon and wishbonearray sensor designs. Again, the basic concept of improving sensitivity by increasing the surface area at anti-nodal locations to boost the interaction of the sensor with the environment and prompt the selective congregation of loading material can be applied to other device architectures. The varying feature density design here again adopts the structural advantages of an open, mesh-like architecture in fitting with the envisioned application. One disadvantage of this particular design is low signal amplitude. This low signal amplitude is thought to be a result of energy loss in the higher-order bending that is induced in the driving sections during resonant vibration. Lower-order bending or purely extensional vibration is more efficiently coupled with.

The hybrid ribbon design featured in this chapter takes advantage of the coupling efficiency provided by extensional vibration, while still maintaining an open, expandable structure that can be compressed into a delivery configuration for a self-expanding biliary stent monitoring system. For example, a sensor mounted to the fully-connected stent design described in Chapter 4 can survive compression into a $2 \mathrm{~mm}$ diameter tube. After subsequent expansion, the sensor exhibited negligible changes in amplitude and frequency 
response. The signal amplitude from this design is high, and the amplitude remains high even when heavily loaded. Though the sensitivity is somewhat lower than other designs, the full-scale range and wireless range of the hybrid ribbon design exceed those of the other complex designs (i.e. wishbone-array, spatially sensitive, and varying feature density designs). Sensitivity may be improved by incorporating small features at the ends of the design, much like the varying feature density design. The signal amplitude may also be further scalable by adding more ribbons linked together with flexible couplers.

Comparing the results of the gelatin testing with results from paraffin testing provides some worthy insight into the operation of resonant sensors when loaded by materials with different viscoelastic properties. The stiffer paraffin has a significant effect on the characteristic frequency of the sensor response, and a less significant effect on the quality factor of the response. Additionally, the stiffer paraffin tends to have a large effect on the amplitude of the response, especially when it accumulates in the interstices of the sensor pattern. In contrast, the gelatin has a large effect on the quality factor of the sensor response while insignificantly affecting the characteristic frequency. Also, gelatin-filled interstices do not have as major of an effect on sensor signal amplitude. Actual loading materials encountered in the end-use applications of these sensors may be more gelatinlike or more paraffin-like, or could have some combination of the viscoelastic properties of the two materials. Whatever the case may be, the ability to monitor both characteristic frequency and quality factor of the resonant sensor simultaneously should allow useful information about the load (and more importantly the disease progression) to be determined. 
The sensors investigated here used transversely annealed Metglas ${ }^{\mathrm{TM}}$ 2605SA1. As described in section 5.1.1, this material was expected to offer increased amplitude robustness while loaded as compared to other Metglas ${ }^{\mathrm{TM}}$ alloys, while still retaining sufficient frequency response sensitivity to the loads. These expectations were verified with simple ribbon sensors, as described in section 2.2. The results from more complex sensor geometries presented in this chapter offer additional insight into the material selection process. Results of the tests described in this chapter indicate that the amplitude robustness was likely improved. However, one consequence of this material choice is that the sensitivity of the quality factor - which as found in this chapter plays an important role in the measurement of viscosity and viscoelastic damping effects - is lower than it could be with other materials. The transverse field annealing treatment, which lowers the exhibited quality factor of the material (and thus makes it less sensitive to damping), also exacerbates this situation. The degradation in quality factor sensitivity is further compounded by the complex mode shapes of the wishbone-array, spatially sensitive, and varying feature density designs; the bending and transverse movement induced in these mode shapes leads to significant structural damping. Future designs should take into account the need to balance amplitude robustness with sensitivity, and the fact that striking such a balance requires careful material selection, specification of thermal treatment, modal analysis to estimate structural damping, and knowledge of the characteristics of the loading material and environment.

It may be fruitful in future designs to combine each of the concepts that have been demonstrated here into one sensor (or sensor array). For example, the results of the gelatin testing presented in this chapter suggest that significant variability may be 
introduced in the sensor response due to the spatial distribution of the loading material. Because the spatial distribution of the sludge in the end-use application will likely vary on a case-to-case basis, it may be best to deploy an array of sensors, each with sensitivity to accumulation only in one spatial location. This spatial sensitivity may be achieved with the concepts shown in this chapter - namely, tailoring of mode shapes and feature density of the sensor. If an array of such sensors were deployed, each sensitive to loading in separate locations and independently interrogable, the full distribution of sludge may be characterized, and variations in sludge distribution will not be averaged over the entire sensor response. 


\section{Chapter 6}

\section{In Situ and Ex Vivo Testing}

The test results presented in previous chapters provides a portrait of system performance in controlled bench-top testing with testing materials that are meant to simulate the effects of biological materials that will be in play in the envisioned final applications of the system. It is, of course, required to also evaluate system performance using situations and materials that more closely mirror the end-use of the system. This chapter presents the design, fabrication, and experimental results of a system that is used in just such a study. The study consists of the placement of a self-expanding biliary stent, with an integrated magnetoelastic sensor, into a porcine carcass in an anatomically appropriate position. This in situ test will evaluate the viability of the sensor in biological fluids (e.g. blood, bile, and other digestive fluids) while also evaluating the external interrogation coil and measurement interface in the presence of interference from a surgical setup. Figure 6-1 illustrates a conceptual schematic of the system architecture used in this work.

In addition, bile is harvested from the animal and its effects on sensor response are compared to the effects of fluids used in previous bench-top studies. This ex vivo test will allow the evaluation of the sensor response to actual biological fluids, and will verify 
the appropriateness of previous bench-top tests using non-biological fluids assumed to be similar to bile.

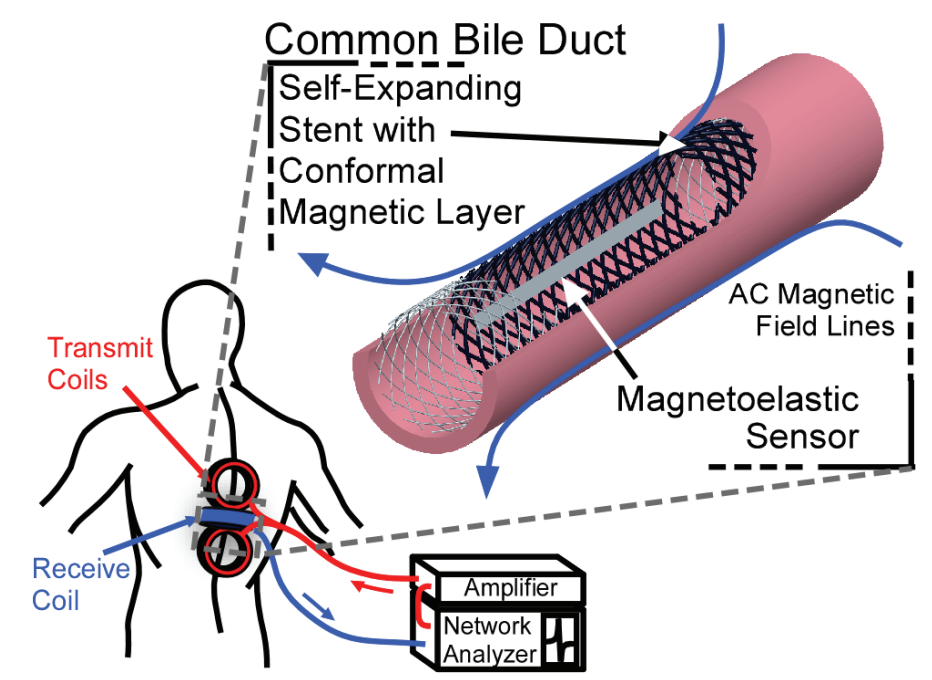

Fig. 6-1: Conceptual sketch of the magnetoelastic monitoring system used in the porcine carcass in situ experiment. A commercial self-expanding biliary stent is modified with a conformal magnetic layer that biases an attached magnetoelastic ribbon sensor.

The goals for this work include exploring the feasibility of integrating a magnetoelastic sensor into a commercial self-expanding stent, rather than into stents designed specifically with magnetoelastic sensing in mind as described in Chapters 3 and 4. The design and fabrication issues associated with the application of a SrFe-PDMS conformal magnetic layer (see Chapter 4) to the commercial stent are also investigated. Survivability of the sensor and magnetic layer after assembly into the delivery system and subsequent deployment is an additional concern addressed here. Finally, the performance of the interrogation module (see section 2.2.4) in a more rigorous setting is evaluated (i.e., in a setting in which the orientation between the sensor and coils, as well as the environment around the sensor, is not predetermined). 


\subsection{Design and Fabrication}

An $8 \mathrm{~mm}$ (diameter) $\times 60 \mathrm{~mm}$ Boston Scientific Wallstent ${ }^{\mathrm{TM}}$ is used for this work (Fig. 6-2). This is the smallest and shortest self-expanding metal stent that is typically used, in keeping with the expected small size of the common bile duct of the intended animal subject. Upon acquisition, the stent is mounted inside of a delivery catheter assembly (the distal portion of which can be seen in Fig. 6-2). This assembly, with any necessary modifications, will also be used to deliver the stent and integrated sensor into the animal subject. In essence, the delivery catheter assembly consists of the delivery sleeve and an inner guidewire. The inner guidewire is longer than the delivery sleeve, and a portion of the guidewire extends proximally to the delivery sleeve, allowing the physician to separately grasp the guidewire and delivery sleeve. Thus, the physician can retract the delivery sleeve while the guidewire is held stationary. Features on the guidewire keep the stent from retracting along with the delivery sleeve; thus the stent is slowly exposed and elastically expands to prop open the constricted duct. In order to integrate the magnetoelastic sensor and biasing magnetic layer, the stent is fully deployed from the delivery catheter.

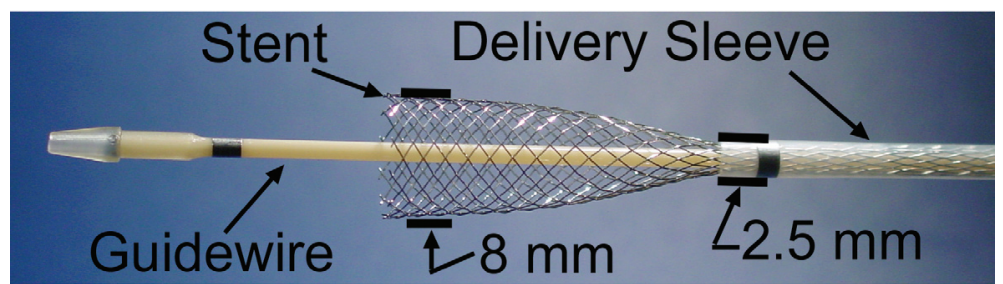

Fig. 6-2: Partially deployed $8 \mathrm{~mm}$ x $60 \mathrm{~mm}$ self-expanding biliary stent (Wallstent ${ }^{\mathrm{TM}}$, Boston Scientific). Subsequent to integration of the conformal magnetic layer and magnetoelastic sensor, the stent is placed back in the delivery system after modification to the delivery sleeve diameter. 
As first described in Chapter 4, a SrFe-PDMS conformal magnetic layer is added to the stent in order to provide a uniform biasing magnetic field to the magnetoelastic sensor. In this work, it is found that the larger outer diameter of the stent compared to the previously studied stents results in a lower bias field for a given SrFe-PDMS ratio and layer thickness. As such, a 3:1 SrFe-PDMS ratio is used, applied in a $225 \mu \mathrm{m}$ thick layer. Because the stent is already in a tubular shape, as opposed to the initially planar stents described in Chapter 4, the stent coating process is slightly modified for this work. First, the SrFe-PDMS mixture is spread in a thin layer of an approximately uniform $100 \mu \mathrm{m}$ thickness on a planar substrate. The ends of the stent are masked using thin adhesive tape, and then the stent is rolled along the SrFe-PDMS layer. As the stent struts contact the SrFe-PDMS, the magnetic composite adheres to the struts in a thin layer. The magnetic composite is then cured at $150^{\circ} \mathrm{C}$ for 45 minutes. Additional coats can be used to build up the thickness of the layer (and increase the resulting magnetic field). For example, two coats covering the middle $30 \mathrm{~mm}$ of the length of the stent and applied in this manner result in a 1.1 Oe field from a layer approximately $150 \mu \mathrm{m}$ thick, while the application of three coats results in a 1.65 Oe field from a layer approximately $225 \mu \mathrm{m}$ thick as used in the final device. Note that the mechanical properties of this thick, high $\mathrm{SrFe}$ content layer are less than ideal, especially in terms of repositioning the stent into the delivery system. This will be discussed further shortly.

For simplicity, a $1 \mathrm{~mm} \times 25 \mathrm{~mm}$ magnetoelastic ribbon sensor is chosen for this investigation. The sensor is fabricated from Metglas $^{\mathrm{TM}}$ 2605SA1 using the photochemical machining process (see section 3.1.1). To improve the signal amplitude and lower the required biasing field, the sensor is transverse field annealed using the 
same process described in section $5.2\left(1\right.$ hour at $413^{\circ} \mathrm{C}$ in a $1.5 \mathrm{kOe}$ transverse magnetic field). Because the stent is already fabricated (and has not been designed with sensor attachment in mind), no assembly features are present. Although some form of assembly feature could be included in the design of the sensor (e.g. a clip, anchors or tether as described in Chapters 3 through 5), the sensor can also be bonded to the stent using an appropriate adhesive applied at the mid-length (vibrational node) of the sensor. For this work, the sensor is bonded to the coated stent using a small amount of PDMS, which is cured at $100^{\circ} \mathrm{C}$ for 1 hour. A coated stent with an integrated sensor is shown in Fig. 6-3.

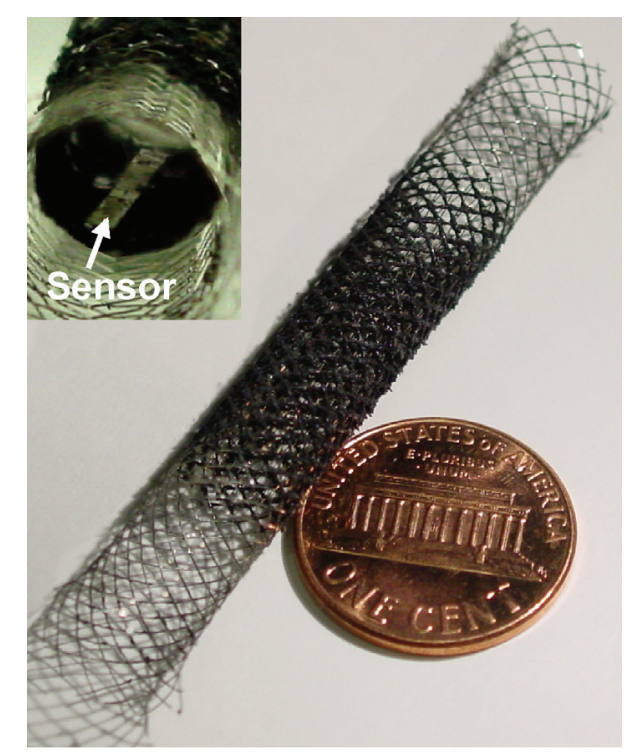

Fig. 6-3: Biliary stent after integration of sensor and conformal magnetic layer. The inset shows a view of the stent lumen and attached sensor. The ends of the stent are masked during the conformal magnetic layer coating procedure.

After coating the stent and integrating the sensor, the assembly must be placed back into the delivery system. Ideally, the system would fit into the same $\sim 2.5 \mathrm{~mm}$ delivery catheter in which the stent was initially mounted. Unfortunately, the thick, high $\mathrm{SrFe}$ content magnetic layer affects the compressibility of the assembly and adds significant 
challenge to fitting the system back into the same diameter delivery catheter. For the purposes of this work, a $4 \mathrm{~mm}$ diameter delivery catheter replaces the original delivery catheter. Trial assembly and deployment of the coated stent with integrated sensor showed that the sensor signal remained consistent from before to after assembly and deployment. Also, the stent continued to reach its fully expanded diameter despite the presence of the stiff conformal magnetic layer.

For interrogation of the sensor, the interrogation coils (extracorporeal configuration (C); see section 2.4) are driven and measured by a HP4395A network analyzer, with the transmit signal amplified by a commercial power amplifier (Krohn-Hite).

\subsection{Porcine Carcass Experiment ${ }^{1}$}

The pig was euthanized as part of a separate medical study. All surgical procedures in this experiment were performed by Dr. Richard Kwon. Also as part of the separate study, fluoroscopic pictures were taken of the lower abdomen, and then the abdomen was opened surgically for visual inspection of the internal tissues. This portion of the procedure was completed within $\sim 20$ minutes after euthanization.

The common bile duct was then located. Upon visual inspection, the diameter of the duct was about the same diameter as the original delivery system $(\sim 2-2.5 \mathrm{~mm})$. As noted previously, the delivery system had been modified with a larger diameter $(4 \mathrm{~mm})$ delivery sleeve. The decision was made to attempt to introduce the system into the common bile duct, despite the size mismatch. The bile duct was approached through the orifice in the

\footnotetext{
${ }^{1}$ The author gratefully acknowledges Dr. Richard Kwon and Dr. Grace Elta of the Internal Medicine Department at the University of Michigan for guidance in this research. Access to the carcass and surgical procedures were also provided by Dr. Kwon and are gratefully acknowledged.
} 
small intestine. After numerous attempts, it became clear that the large delivery sleeve would not fit in the duct. As an alternative approach, a portion of the small intestine near the bile duct was clamped so that it formed a tube of approximately $6 \mathrm{~mm}$ in diameter. The stent was then easily delivered into this approximation of a bile duct (Fig. 6-4). Note that, upon delivery into the makeshift bile duct, the sensor was completely covered in blood and other intestinal fluids (as confirmed upon removal of the stent at the close of the test).

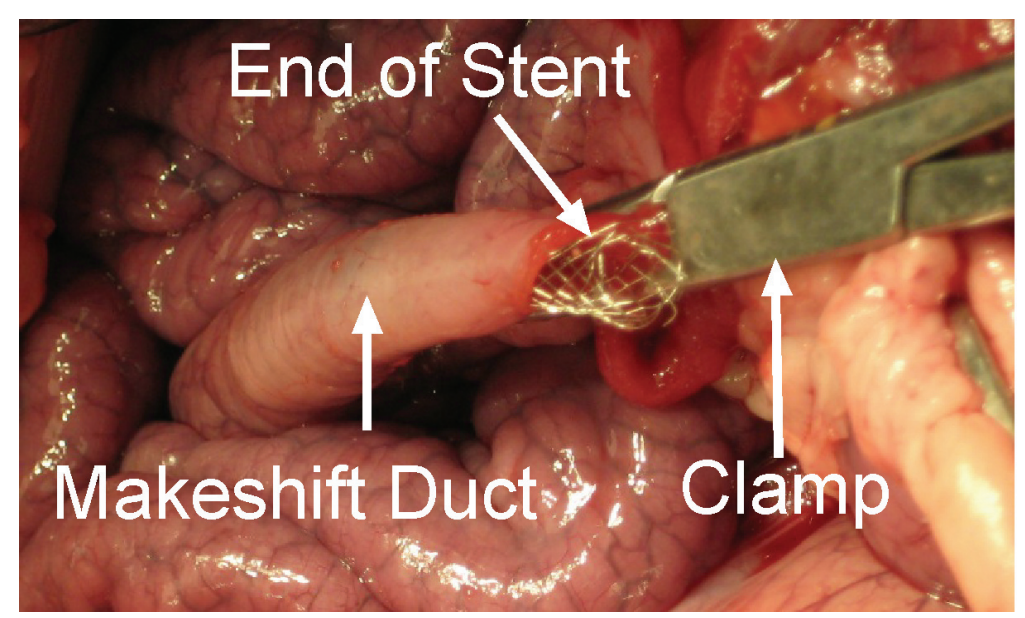

Fig. 6-4: A makeshift biliary duct of approximately $6 \mathrm{~mm}$ in diameter is formed using a portion of the small intestine and a clamp. The modified stent is then deployed in the duct, with the proximal portion protruding as shown here.

As described in section 2.4.3, shifts in the baseline coupling between the transmitted and received signals due to the presence of medical furniture occur which may wash out the sensor signal. Because the animal was positioned with its dorsal side on a surgical table, the possibility of these baseline shifts was high. In fact, such shifts did occur when the baseline was taken well away from the pig and then the coils were positioned near the pig. In an attempt to minimize this baseline shift, the coils were stabilized over the 
animal subject on over-hanging metal tray tables. Then, the pig was lowered (on the moveable surgical table) away from the coils, such that the sensor was approximately 8 inches away from the face of the coils, and a baseline was taken. The pig was then moved back up to the coils. Figure 6-5 shows the basic arrangement of the coils and animal during this procedure. The process described here resulted in a repeatable baseline, such that the sensor response could be measured.

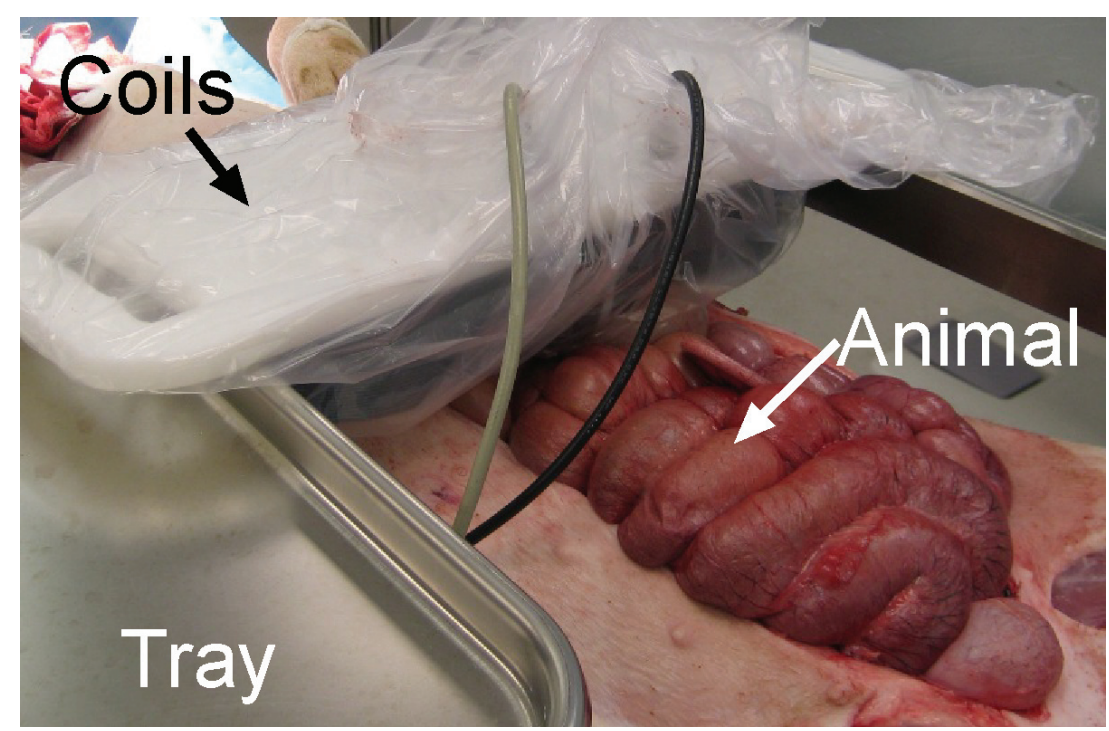

Fig. 6-5: The coils are stabilized over the animal using overhanging metal trays. The animal is lowered away from the coils so a baseline can be measured. The animal is then raised back into position for interrogation of the sensor.

Figure 6-6 shows the measured sensor amplitude response, acquired when the sensor was approximately $5 \mathrm{~cm}$ away from the face of the coils, inside the pig. Figure 6-7 shows the measured sensor amplitude response, acquired when the sensor was approximately $2 \mathrm{~cm}$ away from the face of the coils, inside the pig. Note that a significant baseline shift still exists in both cases, but the sensor response is discernible from the baseline shift. The data can be analyzed after acquisition to remove the majority 
of the baseline shift and improve the visibility of the sensor response, as shown in Figures 6-6B and 6-7B.
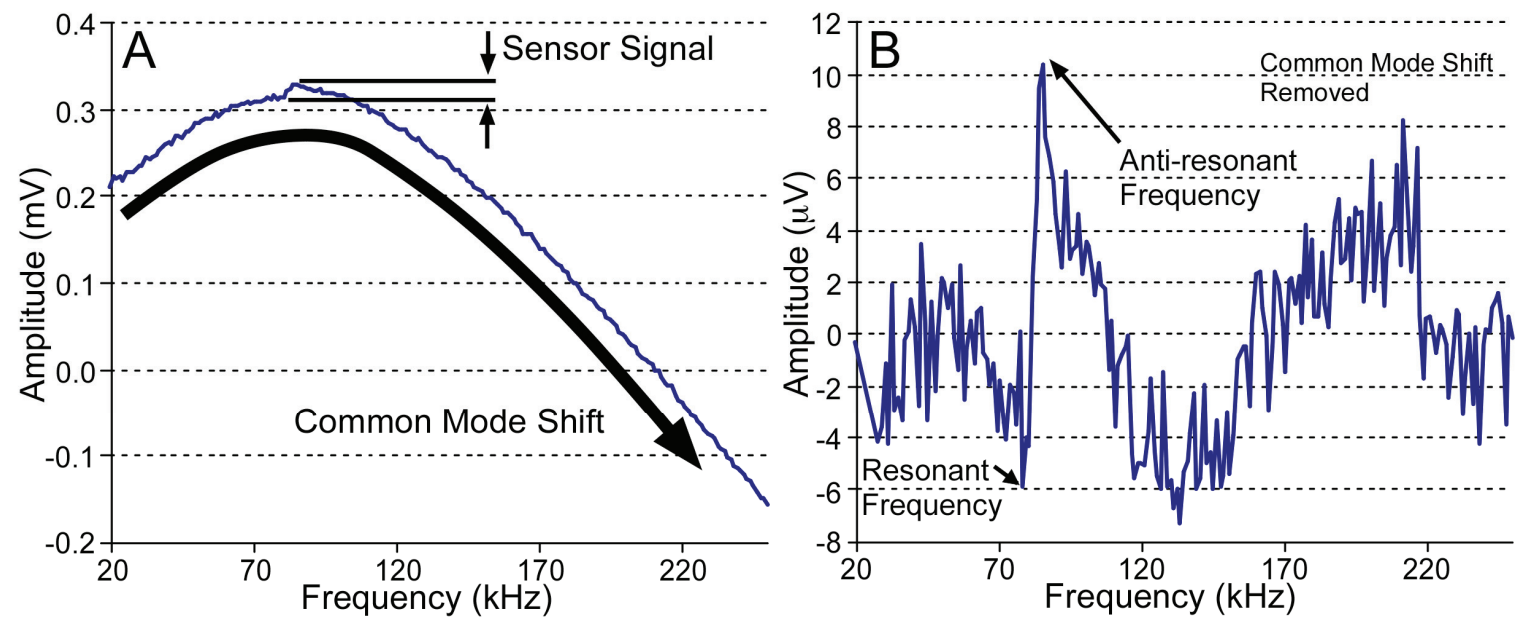

Fig. 6-6: A) Measured amplitude shift from baseline for the implanted sensor at a distance of $5 \mathrm{~cm}$ from the face of the coils. Note the small sensor signal riding on top of a larger common mode shift. B) The common mode shift can be removed in a postprocessing step, making the sensor response more visible. After post-processing, the signal-to-noise ratio is between 2 and 4.
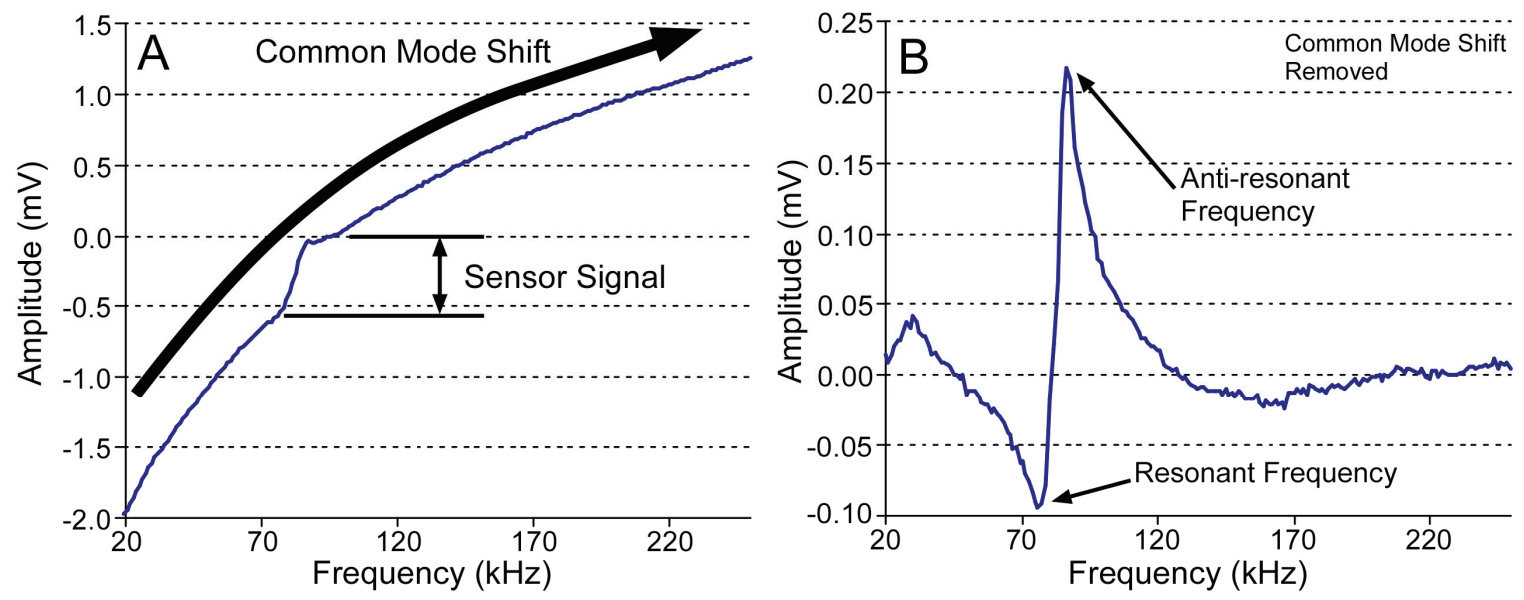

Fig. 6-7: A) Measured amplitude shift from baseline for the implanted sensor at a distance of $2 \mathrm{~cm}$ from the face of the coils. Note the sensor signal riding on top of a larger common mode shift. B) The common mode shift can be removed in a postprocessing step, making the sensor response more visible. The sensor signal is $\sim 18 \mathrm{x}$ larger than that measured in Fig. 6-6 due to the proximity of the sensor, as expected. However, measured anti-resonant and resonant frequencies are identical, also as expected. 


\subsection{Harvested Bile Characterization}

\subsubsection{Viscous Effects of Bile}

To evaluate the response of the sensor in actual, end-use biological fluids, approximately $20 \mathrm{~mL}$ of bile was harvested from the liver of the animal and used in a bench-top setting. A $1 \mathrm{~mm}$ wide $\times 25 \mathrm{~mm}$ long $\times 25 \mu \mathrm{m}$ thick ribbon sensor $\left(\right.$ Metglas $^{\mathrm{TM}}$ 2605SA1), that had been photochemically machined and transverse field annealed (1 hour at $413^{\circ} \mathrm{C}$ in a $1.5 \mathrm{kOe}$ transverse magnetic field), was used to estimate the viscosity of the bile. First, the response of the sensor was calibrated using fluids of known density and viscosity (Dow Corning 200 fluid $(2 \mathrm{cS}, 5 \mathrm{cS}, 10 \mathrm{cS}, 20 \mathrm{cS}$ ) and DI water). The sensor was then immersed in the bile (at room temperature) and the response was measured, with the phase dip half-power bandwidth $(\Delta f)$ over the phase dip center frequency $\left(f_{o}\right)$ being the relevant measurement (as described in section 2.2.2.2). This parameter was used to back-calculate the square root of the density-viscosity product using the calibration curve. Assuming the density of bile is near that of water, the viscosity of bile is estimated to be between 2.75-3.68x that of water (Fig. 6-8), which is in good agreement with literature [Jun01]. Note that this test shows that the range of fluids that has been used in other tests in this work appropriately reflects the viscous effects of bile. 


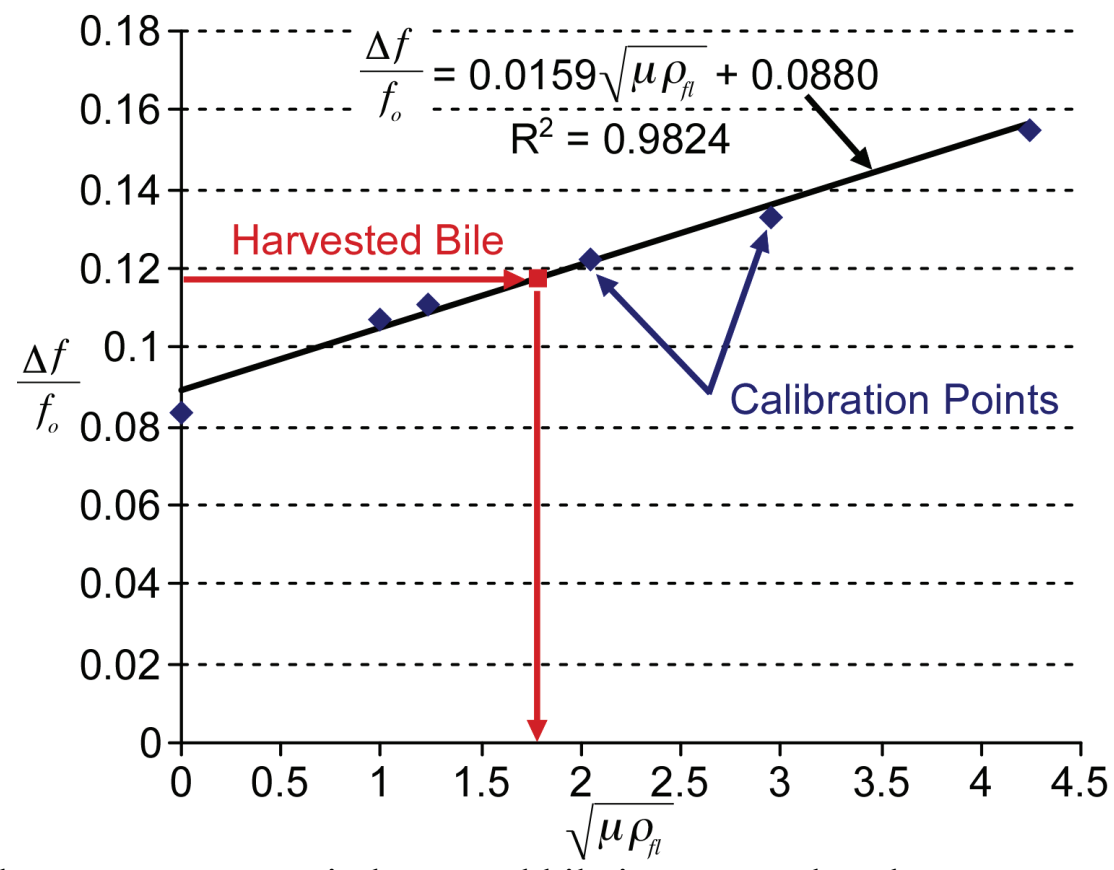

Fig. 6-8: The sensor response in harvested bile is compared to the response of the sensor in fluids of known properties, showing that the viscosity of the bile can be estimated and is within the range of fluids used in previous tests.

\subsubsection{Effects of Bile in Conjunction with a Sludge Simulant}

Finally, the same $1 \mathrm{~mm} \times 25 \mathrm{~mm}$ ribbon sensor was placed in a $6 \mathrm{~mm}$ (inner diameter) plastic tube, which was then filled with a 3:1 by-weight ratio mixture of $500 \mathrm{cS}$ silicone fluid (Dow Corning) and polyvinyl butyral powder to act as a sludge simulant. Then, a rough 3-4 mm diameter open flow channel was made in the sludge simulant. The response of the sensor while placed in the tube and loaded by sludge simulant was first measured in air and then while immersed in bile. The response of the sensor loaded by sludge simulant was different from the response of the unloaded sensor, including a decrease in both quality factor and characteristic frequency. The simulated sludge loading also produced a much larger magnitude of shift in the sensor response than did the immersion of the unloaded sensor in bile. However, no statistically significant difference was seen between the response of the loaded sensor in air and the response of 
the loaded sensor immersed in bile. This comparison included measurements of signal amplitude, phase-dip center frequency, phase-dip half-power bandwidth, and resonant frequency. It is thought that the presence of the highly viscous sludge simulant shields the sensor from the viscous effects of the fluid in the flow channel. This effect makes physical sense due to the decay of the shear waves as the vibrations pass through the viscous sludge stimulant [Dar01]. In the end-use application, this shielding effect is of little consequence, because the knowledge of the presence (and amount) of sludge is more desirable information than knowledge of shifts in viscosity once sludge has begun to accumulate.

\subsection{Discussion}

Integration of the sensor and conformal magnetic layer with a commercial biliary stent presented new challenges that were partially addressed with this work. The most significant challenge encountered was the difficulty in fully compressing the assembly into the original delivery system due to the added stiffness and bulk of the thick, high SrFe content conformal magnetic layer. For this work, the diameter of the delivery system was increased to accommodate the assembly. This allowed for the realization of

the desired system: a wireless resonant magnetoelastic sensor integrated with a commercial self-expanding biliary stent and biased only with the integrated magnetic layer. However, increasing the diameter of the delivery system may not be feasible in the final application because of the limited size of endoscopic tools used to guide the delivery 
system. Improvement may be possible through the development of better assembly tools or through the development of a thinner, more compliant magnetic layer.

The implantation of the enhanced stent into a pig carcass allowed for the confirmation of a measurable sensor response in a situation that mirrors the envisioned end-usage. Although a shift in the baseline coupling between the transmit and receive coils did occur (likely as a result of the presence of the surgical table on which the animal was positioned), a response from the sensor was measurable with at least $5 \mathrm{~cm}$ of wireless range. In the future, testing can be expanded to include measurement of the deployed sensor response as the stent is perfused with relevant materials like bile, blood, or sludge. In this work, bile was harvested from the animal and its effect on the sensor response was compared with effects on the sensor response from fluids of known properties. The results of this comparison verified the suitability of fluids used as bile substitutes in previous tests. Overall, the results of the experiments described in this chapter demonstrate the fundamental usability of the sensor in in situ and ex vivo contexts. 


\section{Chapter 7}

\section{Conclusions and Future Work}

The previous chapters document the first reported efforts toward a wireless magnetoelastic system for monitoring biliary stents. As described in Chapter 2, the components of such a system - including the stent, the magnetoelastic sensor, the biasing magnet, and the interrogation module - should possess certain performance characteristics as determined by the application. This work has presented a varied set of designs of each of these components, and in general each design has exhibited specific strengths and weaknesses. However, the performance of each design can be used to paint a portrait of the feasible performance space. The best performance achieved for each of these components against the desired performance goals has been summarized in Table 71 , along with the location in this work in which the performance is described in more detail (for easy reference).

Section 7.1 summarizes the strengths and weaknesses of the various designs with regards to performance, along with describing some design possibilities that could be realized in the near-term to improve the performance of each component for this application. Section 7.2 describes some recommended future work that could advance the state of the technology even further, not only for the biliary stent application that has been the primary focus of this work, but also for other exciting applications. Finally, the 
major contributions of this work to two general fields - wireless magnetoelastic resonant

devices and applications for bulk-metal micromachining processes - are summarized in

section 7.3.

Table 7-1: Component performance summary

\begin{tabular}{|c|c|c|c|c|}
\hline $\begin{array}{l}\text { System Component and } \\
\text { Performance Category }\end{array}$ & Performance Goal & $\begin{array}{l}\text { Best Measured } \\
\text { Performance }\end{array}$ & Design & Refer to: \\
\hline \multicolumn{5}{|l|}{ - Stent } \\
\hline Bending Flexibility & $\begin{array}{l}<25 \% \text { lumen loss } \\
\text { when bent around } 5 \\
\text { cm diameter }\end{array}$ & $\begin{array}{l}25 \% \text { lumen loss around } \\
5 \mathrm{~cm} \text { diameter }\end{array}$ & Switchback stent & $\begin{array}{l}\text { Section } \\
4.4 .3\end{array}$ \\
\hline Radial Stiffness & $\begin{array}{l}0.1-0.5 \mathrm{~N} / \mathrm{mm} \\
\text { measured at ends and } \\
\text { middle of stent length }\end{array}$ & $0.06-0.18 \mathrm{~N} / \mathrm{mm}$ & $\begin{array}{l}\text { Fully-connected } \\
\text { stent }\end{array}$ & $\begin{array}{l}\text { Section } \\
4.4 .3\end{array}$ \\
\hline \begin{tabular}{|l|} 
Self-Expansion \\
(Expanded \\
Diameter/Delivery \\
Diameter)
\end{tabular} & $\begin{array}{l}\text { At least 3:1 }(200 \% \\
\text { expansion) }\end{array}$ & $4: 1$ & Switchback stent & $\begin{array}{c}\text { Section } \\
4.4 .3\end{array}$ \\
\hline \multicolumn{5}{|l|}{ - Sensor } \\
\hline Full Scale Range & $\begin{array}{l}\text { Down to } 2 \mathrm{~mm} \text { of } \\
\text { patent diameter }\end{array}$ & $\begin{array}{l}\text { Fully occluded (gelatin } \\
\text { sludge simulant, } 6 \mathrm{~mm} \\
\text { tube) }\end{array}$ & $\begin{array}{l}\text { Hybrid ribbon } \\
\text { design }\end{array}$ & $\begin{array}{l}\text { Section } \\
5.4\end{array}$ \\
\hline Resolution & $\begin{array}{l}2 \mathrm{~mm} \text { of diameter } \\
\text { patency }\end{array}$ & $\begin{array}{l}2 \mathrm{~mm} \text { of diameter } \\
\text { patency within a test run }\end{array}$ & $\begin{array}{l}\text { Hybrid ribbon } \\
\text { design }\end{array}$ & $\begin{array}{l}\text { Section } \\
5.4\end{array}$ \\
\hline $\begin{array}{l}\text { Compressibility/ } \\
\text { Expandability }\end{array}$ & $\begin{array}{l}\text { Survive compression } \\
\text { into } 2.5 \mathrm{~mm} \text { diameter } \\
\text { delivery catheter }\end{array}$ & $\begin{array}{l}\text { Compression into } 2 \mathrm{~mm} \\
\text { diameter tube }\end{array}$ & $\begin{array}{l}\text { Hybrid ribbon } \\
\text { design }\end{array}$ & $\begin{array}{l}\text { Section } \\
5.5\end{array}$ \\
\hline $\begin{array}{l}\text { Longitudinal Curvature } \\
\text { Accommodated }\end{array}$ & $\begin{array}{l}70 \mathrm{~mm} \text { or smaller } \\
\text { curvature radius with } \\
\text { no change to } \\
\text { amplitude or } \\
\text { frequency }\end{array}$ & $110 \mathrm{~mm}$ curvature radius & $\begin{array}{l}\text { Ribbon sensor } \\
\text { design }\end{array}$ & $\begin{array}{l}\text { Section } \\
2.2 .5\end{array}$ \\
\hline $\begin{array}{l}\text { Signal Change After } 12 \\
\text { Months Simulated } \\
\text { Implantation }\end{array}$ & $\begin{array}{l}<0.5 \% f_{0} \\
<20 \% \text { Amplitude }\end{array}$ & $\begin{array}{l}<0.5 \% \mathrm{f}_{\mathrm{o}},+/-20 \% \\
\text { Amplitude, } 12 \text { months } \\
\text { accelerated testing }\end{array}$ & $\begin{array}{l}\text { Ribbon sensor } \\
\text { design }\end{array}$ & $\begin{array}{l}\text { Section } \\
2.2 .4\end{array}$ \\
\hline \multicolumn{5}{|l|}{ - Bias Magnets } \\
\hline $\begin{array}{l}\text { Bias Field Provided over } \\
\text { Sensor Length }\end{array}$ & Up to $5 \mathrm{Oe}$ & $1.65 \mathrm{Oe}$ & $\begin{array}{l}225 \mu \mathrm{m} \text { thick } \\
\text { conformal layer } \\
\text { (3:1 SrFe:PDMS } \\
\text { ratio) }\end{array}$ & $\begin{array}{l}\text { Section } \\
6.1\end{array}$ \\
\hline $\begin{array}{l}\text { Change in Stent } \\
\text { Stiffness/Expandability }\end{array}$ & No significant changes & No significant changes & $\begin{array}{l}100 \mu \mathrm{m} \text { thick } \\
\text { conformal layer } \\
\text { (1:1 SrFe:PDMS } \\
\text { ratio) }\end{array}$ & $\begin{array}{l}\text { Section } \\
4.4 .2\end{array}$ \\
\hline \multicolumn{5}{|l|}{ - Interrogation Module } \\
\hline Signal-to-Noise Ratio & $\begin{array}{l}\text { At least } 4 \text { at Full Scale } \\
\text { Range of sensor }\end{array}$ & $\begin{array}{l}77, \text { Hybrid ribbon } \\
\text { sensor, gelatin sludge } \\
\text { simulant, fully occluded } \\
6 \mathrm{~mm} \text { tube } \\
\end{array}$ & $\begin{array}{l}\text { Coil } \\
\text { configuration (C) }\end{array}$ & $\begin{array}{l}\text { Section } \\
5.4\end{array}$ \\
\hline Integration Time & $<10$ seconds & 16 seconds & All tests & \\
\hline \begin{tabular}{|l|} 
Accommodation of \\
Interrogation-Module-to- \\
Sensor Misalignment
\end{tabular} & $\begin{array}{l}+/-5 \mathrm{~cm} \text { in lateral } \\
\text { directions with } 80 \% \text { of } \\
\text { signal amplitude and } \\
\text { no change in resonant } \\
\text { frequency; }>7.5 \mathrm{~cm} \\
\text { implant depth }\end{array}$ & $\begin{array}{l}+/-3 \mathrm{~cm} \text { in lateral } \\
\text { directions }\end{array}$ & $\begin{array}{l}\text { Coil } \\
\text { configuration (C) }\end{array}$ & $\begin{array}{l}\text { Section } \\
2.4 .2\end{array}$ \\
\hline
\end{tabular}




\subsection{Component Performance Summary and Potential Design Improvements}

\subsubsection{Stents}

The designs presented in this work have been shown to offer mechanical performance comparable to that of commercially available self-expanding metal biliary stents. Expandability of $200-300 \%$ is maintained by using elongated mesh patterns and an appropriate material choice. Bending flexibility of the pattern is enhanced through the use of switchback linkages between columns of the pattern, allowing the stent to be wrapped around a $5 \mathrm{~cm}$ diameter cylinder with only a $25 \%$ loss in lumen. Radial stiffness of these designs is shown to be in the same $0.1-0.5 \mathrm{~N} / \mathrm{mm}$ range offered by commercial stents. All of these results have been achieved while also offering a design with minimal length change when expanding $(<10 \%$ length change compared to the $>50 \%$ of the Boston Scientific Wallstent ${ }^{\mathrm{TM}}$ ).

The use of photochemical machining, especially applied to highly elastic metals like Elgiloy ${ }^{\mathrm{TM}}$, has opened up the possibility for including intricate design features in a selfexpanding stent pattern while maintaining a batch-processing approach. Along with the economical advantages that often stem from such batch-processing approaches, the photochemical machining process also allows for inclusion of features that facilitate seam closure and, more importantly for the monitoring application studied in this work, features that can facilitate sensor integration. Additionally, the planar nature of the process may facilitate the inclusion of the process in many more integrated microsystem applications.

Future development efforts for self-expanding stents as part of this monitoring system should include further investigation of sensor integration possibilities, including welding. 
In future designs, features should be included that ensure that the entirety of the sensor stays near the sidewall of the stent while deployed (especially in cases when the curvature of the bile duct would otherwise result in separation between the sensor and stent sidewall). Additionally, the interaction between small features on the stent and sensors during compression into and deployment from the delivery system should be more fully investigated to ensure that this process does not result in entangled or broken sensors. Finally, modifications to the design of the delivery system within the constraints of endoscopic procedures may also be necessary to fully accommodate the stent, sensor(s), and biasing magnet(s).

Another aspect of biliary stent technology that has only been cursorily examined with this work is the use of the monitoring system in polymeric biliary stents. Although the majority of the design work required for using the system in plastic stents lies in the sensor and magnet components, alternative stent designs and fabrication methods that would facilitate sensor integration could also be investigated.

\subsubsection{Sensors}

The sensor designs presented in this work are varied and each exhibits desirable features while also exhibiting certain disadvantages when viewed in the context of this application. The ribbon sensor design detailed in Chapter 3 is attractive because of simplicity and robust signal amplitude; however, the ribbon structure does not offer optimal flexibility and expandability that is important in self-expanding metal stent applications. A wishbone-array design with better expandability is presented in Chapter 4. While potentially more suitable than the ribbon design to withstand the mechanical 
needs of the deployment process, the signal amplitude from the design (and the associated wireless and full-scale range) is not as robust as the ribbon sensor. The advantages of each design type may best be captured using a hybrid ribbon sensor design as presented in Chapter 5. This design uses flexible linkages to connect narrow ribbons, resulting in a good combination of expandability and signal strength. However, the sensitivity of the design is found to be less than that of the other designs; additional features (such as those described in the varying feature density design in Chapter 5) may improve this weakness. Overall, the sensor designs presented here and the techniques described for evaluating their response provide an indication that crucial performance goals can be met, including the ability to provide a wireless signal in a fully occluded bile duct and to discern the difference between a partially and fully-occluded duct.

The performance of the sensors may be further improved with design work in a few key areas. First, the predictive qualities of the finite element analysis tool presented in this work may be further improved with incorporation of some second-order effects including frequency and feature-size dependence of magnetoelastic material properties, hysteresis, and eddy current losses. Additionally, the material property data base should be continuously built, especially for materials that have undergone various fabrication processes and treatments.

Second, the available magnetoelastic materials should continue to be evaluated (in conjunction with evaluation of thermal treatments of the materials). One particular concern in material selection is the balance between signal amplitude robustness and frequency response sensitivity that is determined by the quality factor of the resonator. An interesting possibility here that should be further explored is the use of transversely 
annealed Metglas ${ }^{\mathrm{TM}} 2826 \mathrm{MB}$ at a biasing field that does not deliver the highest amplitude response, but delivers sufficient amplitude along with a slightly lower quality factor. This behavior has been seen with some preliminary tests, and could result in the ideal balance of material properties. An additional possibility here is transverse field annealing of the bulk material prior to sensor patterning. Although this would require mask alignment based on the direction of the transverse field, the resulting material properties may be more consistent and less dependent on sensor geometry.

Third, methods for integrating the sensor with the stent should continue to be developed and tested, especially with regards to ease and mechanical strength. Included in this effort could be further development of methods for integrating the sensor with plastic stents. Sensor designs specific for polymeric stents (taking into account the smaller diameter and the reduced requirement for expandability) should also be developed if necessary, as polymeric stents make up a large portion of all biliary stents deployed and frequently occlude due to sludge accumulation.

\subsubsection{Bias Magnet(s)}

The work presented in the previous chapters provides a strong case for the integration of the bias magnet(s) with the stent to ensure that the sensor is provided with a consistent, uniform bias field. This design architecture reduces the variability in the sensor response (due to the $\Delta \mathrm{E}$ effect) that may be generated by applying a bias field to the sensor with a potentially misaligned external source. The discrete neodymium magnets used in the work presented in Chapter 3 can provide a large (at least 3 Oe) and reasonably uniform bias field; however, this approach results in undesirable local stiffening of the stent 
structure, along with large field gradients (and associated magnetic forces) near each discrete magnet. The conformal SrFe-PDMS magnetic layer presented in Chapters 4 and 6 is somewhat less capable in generating a large field (the largest achieved field was 1.65 Oe), but it is much more suitable for the mechanical needs of the application. A thin ferrite-polymer composite magnetic layer may be especially suited for plastic stents. The smaller diameter of the stents should increase the achievable magnetic field, and the flexibility of the layer should match well the flexibility of the plastic stents.

The work presented in Chapter 6 does point out a shortcoming of the SrFe-PDMS conformal magnetic layer, and that is the added bulk that a thick magnetic layer adds to the stent, reducing the compressibility of the design. To improve this weakness, there are a few design options that can be investigated. First, alternative ferrites or magnetic particles with stronger remanent properties could be used in the composite magnet. If this still proves insufficient, a thin layer of magnetic alloy (e.g. platinum-cobalt or Permalloy) could be sputtered or electrodeposited onto the stent. If this latter option is employed, care should be taken to ensure delamination does not occur (especially as the stent is compressed and redeployed) and that thin-film stresses do not appreciably affect the mechanical properties of the stent.

\subsubsection{Interrogation Module}

The interrogation module described in this work consists of a network analyzer, amplifier, and interrogation coils. The bulk of the design and characterization work presented is focused on the coil configurations, and the important configurations in the context of the biliary stent monitoring application are the extracorporeal configurations 
(B) and (C). Both of these configurations operate with the same basic concept, and that is the spatial decoupling of the transmit signal from the received signal by placing the receive coils in null points of the transmitted signal. Note that the design changes from configuration (B) to configuration (C) resulted in a $2 x$ improvement in signal-to-noise ratio, while also modestly improving the misalignment that can be tolerated between the sensor and coils.

Assuming the same approach to sensor interrogation is taken, improvements in the coils will require investigating sizes, number of windings, orientations and wire or core materials. One example of a potential improvement to the coils would be the addition of receive coils at other locations (still in null points of the transmitted signal) that would allow for further misalignment between the sensor and coils. Because anatomy will vary from patient-to-patient and because the acquisition of signal from an implanted sensor is essentially blind, tolerance of a large sensor-to-coil misalignment would significantly enhance the usability of the system.

Of course, other approaches for decoupling the transmitted signal from the received signal may also be taken. One of the main drawbacks of the current approach is the potential for baseline shifts due to the presence of various conductive materials (including tissue and medical furniture; see section 2.4.2 and Chapter 6). Modifications to the current approach may be necessary to alleviate this potential.

One such modification is tighter control of the environment around the coils. For example, the coils may need to be stationary and away from structural objects while a baseline is taken. Then, the patient can be placed in the measurement area. To be effective, this method would require consistent baseline shifts in the presence of tissue 
across a wide patient population. It would also require a fairly large tolerance for misalignment between the sensor and coils, such that patients of all shapes and sizes could be accommodated with a stationary coil position. This method is straightforward in terms of design, but relies on as yet undetermined information regarding the patient population.

A second potential modification for alleviating baseline shifts involves turning the sensor "on" and "off" through the use of an externally applied magnetic field. In this approach, a baseline is taken while the coils are in the final measurement position with the sensor nearby. However, when taking the baseline, a "large" DC magnetic field can be generated on top of the small transmitted field (an electromagnet can be used). This DC field is intended to shift the operating point of the sensor so that it is "off". Thus, a baseline can be taken without the resonant response of the sensor, but with the presence of all conductors that affect the baseline coupling between the coils. Once a baseline is taken, the large DC field can be turned off, switching the sensor back "on" (with the operating point determined by the integrated biasing magnet(s)). For this approach to work, the DC field must be large enough to turn the sensor "off" regardless of sensor position and orientation, but not so large that the integrated bias magnets are demagnetized.

\subsection{Long Term Future Work}

\subsubsection{Biliary Stent Monitoring}

One of the main long term requirements to demonstrate a successful design for 
wireless monitoring is appropriate verification and validation testing. There are many facets to this testing, but a few of the most important are focused on here. First, a method for appropriate in vitro testing of the system should be developed; in other words, a method that allows for the controlled generation of sludge from bacteria that have been cultured on the stent and sensor under test. This method should also incorporate an ability to directly visualize the amount of sludge accumulation so that sensor response can be appropriately correlated; the visualization may be done with an endoscopic or fiber optic camera. In vitro testing could follow a protocol similar to that described in [Lam86]. The stent and integrated components can be placed in a plastic tube of appropriate material (polyurethane, polyethylene or Teflon) and appropriate diameter. The tube can be perfused with bile, which can be pumped peristaltically with an appropriate flow rate $(0.5-1 \mathrm{~mL} / \mathrm{min})$ and replaced at an appropriate interval (2-3 weeks). In [Lam86], only $\sim 0.06 \mathrm{~mm}$ of "incrustation" was present after 10 weeks by following this protocol. This rate of accumulation is lower than desired; as such, the accumulation may be accelerated by seeding the stent with proteins and concentrating appropriate bacteria (e.g. E. coli) in the bile. The most difficult aspect of the in vitro test may be procuring sufficient amounts of harvested bile; however, it may be possible to use a synthesized culture medium as a substitute.

A second facet of long term performance verification is further animal testing, including in situ work like that in described in Chapter 6 as well as in vivo work. Ideally, the in vivo testing would occur over a long duration (10 weeks -6 months) in order to adequately identify acute and chronic issues associated with the implant and to allow the sludge accumulation process to sufficiently progress. Correlating the sensor response 
with the actual sensor environment may be difficult, but methods like frequent endoscopy, tissue histology, and liver function testing may be useful.

A third facet of long term verification testing includes assessment of long term stability and biocompatibility of the various components (and specifically, the Parylenecoated resonant sensor). As described previously, the amorphous alloys have a tendency to corrode when exposed to conductive liquids. In this work, this has been mitigated with a Parylene coating. In the future, it should be verified that such a coating is sufficient to ensure the stability and biocompatibility of the sensor materials. Parylene is an oft-used solution for medical device coatings [Sta96], so this approach will likely meet with success. However, if it is determined that Parylene does not sufficiently protect the material, other protective coatings may be investigated, or other biocompatible magnetoelastic materials may be required (perhaps Terfenol- $\left.\mathrm{D}^{\mathrm{TM}}[\mathrm{Pou} 06]\right)$.

A final long term concern is the development of software and a user interface to assist the physician in interpreting the response of the sensor. Another aspect of this development may be the replacement of the network analyzer with a more specialized piece of hardware, assuming the specialized hardware is more cost effective. If the cost (and space requirements) of the interrogative equipment can be driven down while improving the usability of the system, in-home monitoring may eventually be feasible.

\subsubsection{Other Applications}

Although this work has focused on the application of wireless magnetoelastic resonant sensors to the monitoring of biliary stents, there are also other potential applications that could be well addressed by wireless magnetoelastic resonators. Within 
a similar arena, the self-expanding stents used in transjugular intrahepatic portosystemic shunts (TIPS, see section 1.1.2) could benefit from being monitored by magnetoelastic sensors. To ensure applicability of the sensors in a TIPS stent, the response of the sensors to pseudo-intimal hyperplasia and thrombi formation should be characterized and compared to the current Doppler ultrasound standards. In general, because the selfexpanding stents used in TIPS are the same as self-expanding biliary stents, a massive design overhaul should not be necessary.

Another application, also within the same arena but with very distinct challenges as compared to the biliary application, is the monitoring of coronary stents. There are numerous important differences between coronary stent and biliary stent applications that would have to be addressed through sensor and system design. First, coronary stents are much smaller (usually about $2 \mathrm{~mm}$ in diameter) than biliary stents. This would require appropriate scaling of the sensor, which may limit signal amplitude (and wireless range). Second, coronary stents are almost exclusively balloon-assisted to realize their deployed diameter. This requires a paradigm shift for the stent design, as well as the desirable properties of the magnetoelastic sensor. To meet the needs of the application, it may be required to also plastically deform the sensor, which would not be achievable with the brittle amorphous alloys focused on in this work. Alternatively, composite magnetoelastic sensors might be needed. Third, coronary stents are subject to high blood flow rate, which brings not only hemodynamic concerns into play, but also the possibility that such flow may have undesirable effects on the resonance of the sensor. Though the challenges are many, coronary stents represent a sizeable portion of the stent market and they offer a fantastic opportunity for saving lives if a real-time wireless monitoring 
system could be developed.

Of course, the use of magnetoelastic transduction need not be limited to sensor applications. The conversion of magnetic energy to mechanical motion could be tapped for wireless generation of ultrasound - in other words, in actuator applications. Development in this area could be applied in a number of ways. In the medical device arena, implants could be developed with on-board ultrasound generators. The compelling aspect of this concept is the potential for therapeutic (or combination therapeutic and diagnostic) devices, rather than simple diagnostic devices as detailed in this work. For instance, on-board wireless ultrasound generators could potentially be used to "break-up" sludge accumulation, or inhibit the adhesion of bacteria to the implant surfaces. They could be used for targeted drug delivery (e.g. microbubble cavitation [Tac01]). Outside of the medical arena, wireless ultrasound generation could also be useful. For instance, ultrasonic rotary motors [Sas93] could make use of magnetoelastic drives for wireless

actuation. Non-destructive evaluation systems [Kun04] could make use of wireless magnetoelastic ultrasound generators that have been placed in difficult-to-access areas or in a scalable array. For a closer look at a preliminary investigation into wireless magnetoelastic ultrasound generation, see Appendix B.

\subsection{Major Contributions to the Field}

This work has been focused on the specific application of wireless magnetoelastic resonant sensors to monitoring of biliary stents. The conceptualization and specification of this new application, as well as drawing comparisons to similar applications, is an 
important part of this work. Knowledge has been gained with regards to the response of resonant magnetoelastic sensors to viscous and viscoelastic loading, and - importantly methods for interpreting that response have also been demonstrated. In addition, the performance of the magnetoelastic sensors has been pushed to new limits in terms of fullscale range and expandability. Also, the conformal magnetic biasing layer concept developed in this work can be used to address situations where the entire device must remain flexible and compliant as required in biliary stents.

A number of the advances made in the pursuit of a functioning wireless magnetoelastic biliary stent monitoring system have a much more general applicability. The development, implementation, and preliminary experimental verification of analytical and finite element analysis tools for resonant magnetoelastic sensors is an important step toward feasible predictive design and virtual prototyping of sensors suitable for any number of applications. This work has also illustrated tailored magnetoelastic sensor geometry - far beyond simple ribbon sensors - for enhanced functionality and expanded applicability of the transduction technique. In conjunction with this more complex sensor geometry, photochemical machining has been demonstrated as a viable process for batch-patterned sensors (and stents) with complex geometry. This process could hold promise for other applications requiring bulk-metal microstructures. A final important part of the work presented here is the investigation of thermal treatments of magnetoelastic resonant sensors fashioned from amorphous alloys. Although thermal treatments have been investigated previously, this work is the first in the knowledge of the author to apply the treatments to change the shape of the sensor for better performance in tubular structures, and the first to apply transverse field 
annealing treatments to complicated sensor shapes. The utilization of these advances can have a wide-ranging, generally applicable impact on the design, fabrication, and performance of wireless magnetoelastic systems. 
APPENDICES

- 191 - 


\section{Appendix A}

\section{Derivation and Implementation of Analytical and Finite Element Models}

This appendix will present a derivation for the analytical equations listed in Chapter 2, including intermediate equations. Implementation of the analytical model in MatLab is then illustrated. The full implementation of the magnetomechanical finite element tool in COMSOL is shown, and the results of the two tools are compared for simple geometries.

\section{A.1 Constitutive Equations - Compliance Form}

Both the analytical model and finite element analysis tools rely on the linearized piezomagnetic constitutive equations. For ease in progressing to the one-dimensional analytical model from the full three-dimensional equations, the compliance form of the coupled equations is initially presented:

$$
\left[\begin{array}{l}
\varepsilon_{x x} \\
\varepsilon_{y y} \\
\varepsilon_{z z} \\
\varepsilon_{x y} \\
\varepsilon_{x z} \\
\varepsilon_{y z}
\end{array}\right]=\left[\begin{array}{cccccc}
\frac{1}{E^{\prime}} & \frac{-v_{s}}{E^{\prime}} & \frac{-v_{s}}{E^{\prime}} & 0 & 0 & 0 \\
\frac{-v_{s}}{E^{\prime}} & \frac{1}{E^{\prime}} & \frac{-v_{s}}{E^{\prime}} & 0 & 0 & 0 \\
\frac{-v_{s}}{E^{\prime}} & \frac{-v_{s}}{E^{\prime}} & \frac{1}{E^{\prime}} & 0 & 0 & 0 \\
0 & 0 & 0 & \frac{1+v_{s}}{E^{\prime}} & 0 & 0 \\
0 & 0 & 0 & 0 & \frac{1+v_{s}}{E^{\prime}} & 0 \\
0 & 0 & 0 & 0 & 0 & \frac{1+v_{s}}{E^{\prime}}
\end{array}\right]\left[\begin{array}{c}
\sigma_{x x} \\
\sigma_{y y} \\
\sigma_{z z} \\
\sigma_{x y} \\
\sigma_{x z} \\
\sigma_{y z}
\end{array}\right]+\left[\begin{array}{ccc}
d^{\prime} & 0 & 0 \\
0 & d^{\prime} & 0 \\
0 & 0 & d^{\prime} \\
0 & 0 & 0 \\
0 & 0 & 0 \\
0 & 0 & 0
\end{array}\right]\left[\begin{array}{c}
H_{x} \\
H_{y} \\
H_{z}
\end{array}\right]
$$




$$
\left[\begin{array}{l}
B_{x} \\
B_{y} \\
B_{z}
\end{array}\right]=\left[\begin{array}{cccccc}
d^{\prime} & 0 & 0 & 0 & 0 & 0 \\
0 & d^{\prime} & 0 & 0 & 0 & 0 \\
0 & 0 & d^{\prime} & 0 & 0 & 0
\end{array}\right]\left[\begin{array}{c}
\sigma_{x x} \\
\sigma_{y y} \\
\sigma_{z z} \\
\sigma_{x y} \\
\sigma_{x z} \\
\sigma_{y z}
\end{array}\right]+\left[\begin{array}{ccc}
\mu_{o} \mu_{r}^{\prime} & 0 & 0 \\
0 & \mu_{o} \mu_{r}^{\prime} & 0 \\
0 & 0 & \mu_{o} \mu_{r}^{\prime}
\end{array}\right]\left[\begin{array}{c}
H_{x} \\
H_{y} \\
H_{z}
\end{array}\right] .
$$

In (A-1) and (A-2), subscripts $\mathrm{x}, \mathrm{y}$, and $\mathrm{z}$ denote direction; $\varepsilon$ is strain; $\sigma$ is stress; $H$ is magnetic field; $B$ is magnetic flux density; $v_{\mathrm{s}}$ is Poisson's ratio; $E^{\prime}$ is the Young's modulus (at constant magnetic field); $d^{\prime}$ is magnetostrictivity (at constant magnetic field); and $\mu_{r}^{\prime}$ is permeability (at constant magnetic field). Note that the magnetostrictivity and permeability matrices have been made isotropic for simplicity, rather than adopting the anisotropic relationships described in [IEE71]. Preliminary comparison with models derived with anisotropic matrices showed negligible differences in results, although further investigation may elucidate important differences.

\section{A.2 Analytical Model}

As noted in section 2.2.2, the analytical model uses a ribbon sensor (length $2 L$, width $w$, and thickness $\left.t_{\text {sens }}\right)$ oriented with the length along the x-axis, centered about the origin (i.e. existing on the domain $\mathrm{x}=-L$ to $\mathrm{x}=L$ ) with free-free end conditions. The full (3-D) constitutive equations can be simplified if it is assumed that stress and magnetic field are directed only in the $\mathrm{x}$-direction. Then, the 1-D constitutive equations become

$$
\varepsilon_{x x}=\frac{1}{E^{\prime}} \sigma_{x x}+d^{\prime} H_{x}
$$




$$
\text { and } B_{x}=d^{\prime} \sigma_{x x}+\mu_{o} \mu_{r}^{\prime} H_{x}
$$

The analysis most easily proceeds with the constitutive equations rewritten in terms of strain and flux density, which is straightforward:

$$
\begin{gathered}
\sigma_{x x}=\frac{E^{\prime} \mu_{o} \mu_{r}^{\prime}}{\mu_{o} \mu_{r}^{\prime}-d^{\prime 2} E^{\prime}} \varepsilon_{x x}-\frac{E^{\prime} d^{\prime}}{\mu_{o} \mu_{r}^{\prime}-d^{\prime 2} E^{\prime}} B_{x} \\
\text { and } H_{x}=-\frac{d^{\prime} E^{\prime}}{\mu_{o} \mu_{r}^{\prime}-d^{\prime 2} E^{\prime}} \varepsilon_{x x}+\frac{1}{\mu_{o} \mu_{r}^{\prime}-d^{\prime 2} E^{\prime}} B_{x} .
\end{gathered}
$$

If we let

$$
\begin{array}{r}
\mu_{o} \mu_{r}=\mu_{o} \mu_{r}^{\prime}-d^{\prime 2} E^{\prime}, \\
E_{a p p}=\frac{E^{\prime} \mu_{o} \mu_{r}^{\prime}}{\mu_{o} \mu_{r}^{\prime}-d^{\prime 2} E^{\prime}}, \\
\text { and } \quad d=\frac{d^{\prime}\left(\mu_{o} \mu_{r}^{\prime}-d^{\prime 2} E^{\prime}\right)}{\mu_{o} \mu_{r}^{\prime}},
\end{array}
$$

then (A-5) and (A-6) become the simple forms suggested by equations (1) and (2) in section 2.2.1 and used in the remainder of the derivation of the analytical model:

$$
\begin{gathered}
\sigma_{x x}=E_{a p p} \varepsilon_{x x}-\frac{E_{a p p} d}{\mu_{o} \mu_{r}} B_{x} \\
\text { and } H_{x}=-\frac{d E_{a p p}}{\mu_{o} \mu_{r}} \varepsilon_{x x}+\frac{1}{\mu_{o} \mu_{r}} B_{x} .
\end{gathered}
$$

\section{A.2.1 Newton's Law for Derivation of Equation of Motion}

As shown in Figure A-1, an infinitesimal slice of a loaded sensor of length $d x$ is acted upon by stress through the sensor material, stress through the loading material (sludge), 
and damping forces from the viscous media and from the structural losses in the sensor material itself. These forces contribute to the acceleration of the sensor slice, as well as a small amount of fluid (to be determined later). Per Newton's Second Law, the forces in the $\mathrm{x}$-direction are summed to give the time rate of change of momentum in the $\mathrm{x}$ direction:

$$
\begin{aligned}
& A_{\text {sens }}\left(\sigma_{\text {sens }}(x+d x)-\sigma_{\text {sens }}(x)\right)+A_{\text {sludge }}\left(\sigma_{\text {sludge }}(x+d x)-\sigma_{\text {sludge }}(x)\right) \\
& -F_{\text {struc }}-F_{\text {damp }}=\left(\rho_{\text {sens }} A_{\text {sens }} d x+m_{f l} d x+\rho_{\text {sludge }} A_{\text {sludge }} d x\right) \frac{\partial^{2} u(x, t)}{\partial t^{2}} .
\end{aligned}
$$

In (A-12), $m_{f l}$ is the activated fluid mass per unit length, $F_{\text {struc }}$ is the damping force due to structural losses, $F_{\text {damp }}$ is the damping force due to the surrounding viscous media, and $u(x, t)$ is the displacement of the infinitesimal slice. Note that this model assumes that the strain is constant throughout the sensor and the loading layer.

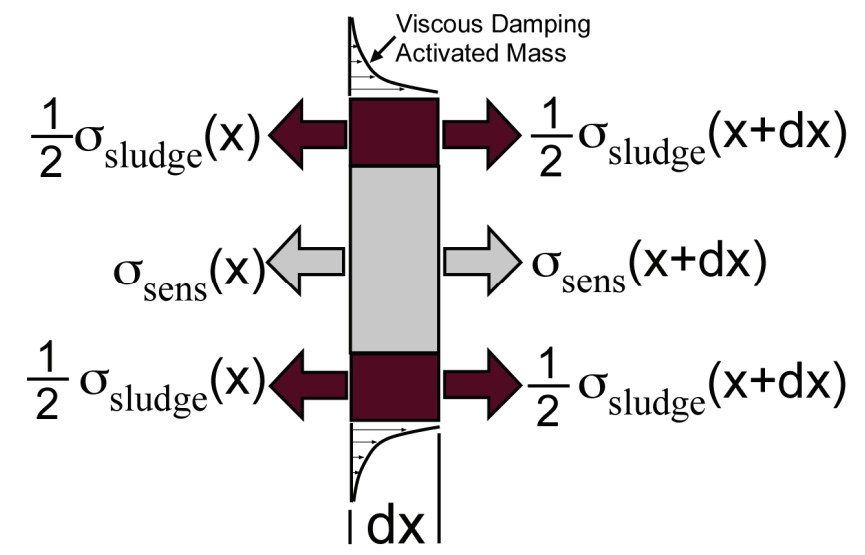

Fig. A-1: Newton's Second Law can be applied to an infinitesimal cross section of the loaded sensor. The sensor also experiences damping due to structural losses, while the fluid provides viscous damping and a small amount of activated mass.

The stress $\sigma_{x x}$ in $(\mathrm{A}-10)$ can be substituted into $\sigma_{\text {sens }}$ in (A-12), along with the following relations: 


$$
\begin{gathered}
\varepsilon_{x x}=\frac{\partial u}{\partial x} \\
\text { and } \sigma_{\text {sludge }}=E_{\text {sludge }}^{*} \varepsilon_{x x}
\end{gathered}
$$

and the equation of motion becomes:

$$
\begin{aligned}
& A_{\text {sens }}\left(E_{\text {app }} \frac{\partial u(x+d x)}{\partial x}-E_{\text {app }} \frac{\partial u(x)}{\partial x}-\frac{E_{\text {app }} d}{\mu_{o} \mu_{r}}\left(B_{x}(x+d x)-B_{x}(x)\right)\right)+ \\
& A_{\text {sludge }}\left(E_{\text {sludge }}^{*} \frac{\partial u(x+d x)}{\partial x}-E_{\text {sludge }}^{*} \frac{\partial u(x)}{\partial x}\right)-F_{\text {struc }}-F_{\text {damp }}= \\
& \left(\rho_{\text {sens }} A_{\text {sens }} d x+m_{f l} d x+\rho_{\text {sludge }} A_{\text {sludge }} d x\right) \frac{\partial^{2} u(x, t)}{\partial t^{2}}
\end{aligned}
$$

\section{A.2.1.1 Damping and Mass Loading Due to Viscous Fluid}

To determine the values of $F_{\text {damp }}$ and $m_{f l}$ in equation (A-15), a treatment presented in [Dar01] is adapted. This treatment assumes harmonic longitudinal motion of the fluidsurface interface, and a no-slip condition at this surface. Thus, the fluid at the surface moves with the same velocity as the surface. It is assumed for the purposes of this treatment that the sensor is long so that end effects are negligible, and that the total thickness of the sensor is negligible compared to the width, such that only the top and bottom surfaces interact with the fluid. With this setup, the fluid momentum equation can be written as

$$
\frac{\partial V_{\text {fluid }}(z, t)}{\partial t}=v \frac{\partial^{2} V_{\text {fluid }}(z, t)}{\partial z^{2}}
$$

subject to the boundary condition 


$$
V_{\text {fluid }}(0, t)=\frac{\partial u}{\partial t}=V_{\text {sens }} e^{i \omega t}
$$

and bounded as $\mathrm{z}$ approaches infinity. In (A-16), $V_{\text {fluid }}$ is the velocity of the fluid in the Xdirection; $v$ is the kinematic viscosity of the fluid; and $\mathrm{z}$ is the distance in the direction normal to the top surface of the sensor, measured from the surface of the sensor. In (A17), the local velocity of the sensor is assumed to be harmonic with magnitude $V_{\text {sens }}$ and radial frequency $\omega$. It can be seen that (A-16) is in the form of the heat-conduction equation with a periodic boundary condition for a semi-infinite domain. We are interested in the steady-state motion of the fluid, i.e. the solution to this boundary value problem as time approaches infinity. Such a solution is well documented. For instance, the treatment in [Cha94] shows that

$$
V_{\text {fluid }}(z, t)=V_{\text {sens }} e^{-2 z \sqrt{\frac{\omega}{2 v}}+i \omega t} \text { for } \mathrm{t} \gg 0 \text {. }
$$

This result can be utilized to find the effective damping force as follows. The fluid damping force is due to shear stress $\tau$ at the surface interface:

$$
F_{\text {damp }}=\tau A_{\text {surface }}=\mu\left|\frac{\partial V_{\text {fluid }}(0, t)}{\partial z}\right| 2 w d x=2 w d x \mu \sqrt{\frac{\omega}{v}} V_{\text {sens }} e^{i \omega t}=2 w d x \mu \sqrt{\frac{\omega}{v}} \frac{\partial u}{\partial t} .
$$

The factor of 2 in (A-19) originates from the fact that the fluid interacts with the top and bottom surfaces of the sensor.

The result in (A-18) can also be used to determine the effective activated fluid mass by determining the kinetic energy of the fluid per unit length and rewriting the kinetic energy in terms of the velocity of the sensor surface: 


$$
K E_{\text {fluid }}=\lim _{z_{f l} \rightarrow \infty} \frac{1}{2} \int_{0}^{z_{f}} 2 \rho_{f l} w V_{\text {fluid }}(z, t)^{2} d z=\frac{1}{2}\left(\frac{1}{2} w \rho_{f l} \sqrt{\frac{2 v}{\omega}}\right)\left(V_{\text {sens }} e^{i \omega t}\right)^{2}
$$

By inspection, the effective mass load per unit length due to the activated fluid is thus

$$
m_{f l}=\frac{1}{2} w \rho_{f l} \sqrt{\frac{2 v}{\omega}} .
$$

\section{A.2.1.2 Structural Damping}

Damping due to losses in the vibrating material is often difficult to predict accurately, and often simple models do not effectively capture the nuances of structural damping including frequency and mode shape dependency. As an initial and simple approach for accounting for structural losses, Rayleigh damping (also known as proportional damping) can be used. This approach is especially useful for the purposes of this derivation because the finite element modeling implementation discussed in section A.3 has built-in capabilities for assigning Rayleigh damping coefficients. For the purposes of the analytical model, the structural damping can be represented as the sum of terms that are proportional to the effective mass and stiffness of the sensor material:

$$
F_{\text {struc }}=\left[\alpha\left(m_{\text {sens }, \text { eff }}\right)-\beta\left(k_{\text {sens,eff }}\right)\right] \frac{\partial u}{\partial t}=\left[\alpha \rho_{\text {sens }} A_{\text {sens }} d x-\beta A_{\text {sens }} E_{\text {app }} \frac{\partial}{\partial x}\right] \frac{\partial u}{\partial t} .
$$

Note the negative sign in front of the term proportional to stiffness; this assures proper direction of the damping force with respect to the direction of strain. 


\section{A.2.2 Eigenfunction Expansion}

The results of (A-19), (A-21) and (A-22) are substituted into (A-15). Then, the width of the sensor $w$ is factored out, and both sides of the equation are divided by $d x$ and the limit is taken as $d x$ goes to zero (using the definition of differentiation), resulting in:

$$
\begin{aligned}
& \left(E_{\text {app }} t_{\text {sens }}+2 t_{\text {sludge }} E_{\text {sludge }}^{*}\right) \frac{\partial^{2} u}{\partial x^{2}}-\left(\rho_{\text {sens }} t_{\text {sens }}+\frac{1}{2} \rho_{f l} \sqrt{\frac{2 v}{\omega}}+2 \rho_{\text {sludge }} t_{\text {sludge }}\right) \frac{\partial^{2} u}{\partial t^{2}} \\
& -\left(2 \mu \sqrt{\frac{\omega}{v}}+\alpha \rho_{\text {sens }} t_{\text {sens }}\right) \frac{\partial u}{\partial t}+\beta t_{\text {sens }} E_{\text {app }} \frac{\partial^{3} u}{\partial x^{2} \partial t}=\frac{E_{\text {app }} d}{\mu_{o} \mu_{r}} t_{\text {sens }} \frac{\partial B_{x}}{\partial x}
\end{aligned}
$$

Equation (A-23) will be used again in the derivation of the voltage induced on a pickup coil in the sensor (section A.2.3). However, to proceed further in the calculation of $u(x, t)$, $B_{x}$ must be replaced in equation (A-23) with $\mathrm{Hx}$ using equation (A-11), as it is the magnetic field that is applied to drive the sensor. It is assumed that $H_{x}$ varies harmonically in time, and is uniform everywhere outside of the sensor. Inside the sensor, $H_{x}$ will vary along the length of the sensor due to the relative permeability of the sensor material. For the purposes of this derivation, the variation in $H_{x}$ along the length of the sensor is captured through the use of a shape function, $\varphi$, the form of which is described later (see section A.2.5). For now, we write:

$$
H_{x}=H e^{i \omega t} \varphi
$$

Thus, (A-23) becomes (after rearranging)

$$
\begin{aligned}
& {\left[\left(E_{\text {app }}-\frac{d^{2} E_{\text {app }}^{2}}{\mu_{o} \mu_{r}}\right) t_{\text {sens }}+2 t_{\text {sludge }} E_{\text {sludge }}^{*}\right] \frac{\partial^{2} u}{\partial x^{2}}-\left(\rho_{\text {sens }} t_{\text {sens }}+\frac{1}{2} \rho_{f l} \sqrt{\frac{2 v}{\omega}}+2 \rho_{\text {sludge }} t_{\text {sludge }}\right) \frac{\partial^{2} u}{\partial t^{2}}} \\
& -\left[2 \mu \sqrt{\frac{\omega}{v}}+\alpha \rho_{\text {sens }} t_{\text {sens }}\right] \frac{\partial u}{\partial t}+\beta t_{\text {sens }} E_{\text {app }} \frac{\partial^{3} u}{\partial x^{2} \partial t}=E_{\text {app }} d t_{\text {sens }} H e^{i \omega t} \frac{d \varphi}{d x}
\end{aligned}
$$

Note that (A-25) is identical to equation (3) in Chapter 2. 
Now equation (A-25), along with the boundary conditions (free ends), is in a form that can be solved. First, the homogeneous version of (A-25) (i.e., in which the righthand side is set to zero) is solved using separation of variables to determine the eigenvalues and associated eigenfunctions, which are:

$$
\begin{aligned}
& \lambda_{n}=\left(\frac{(2 n-1) \pi}{2 L}\right)^{2} \\
& \psi_{n}(x)=\sin \left(\sqrt{\lambda_{n}} x\right)
\end{aligned}
$$

Then, the full non-homogeneous equation (A-25) can be solved using eigenfunction expansion, as follows. Let

$$
\begin{gathered}
u(x, t)=\left[\sum_{n=1}^{\infty} a_{n} \psi_{n}(x)\right] e^{i \omega t} \\
\text { and }\left[\sum_{n=1}^{\infty} q_{n} \psi_{n}(x)\right] e^{i \omega t}=E_{\text {app }} d t_{\text {sens }} H e^{i \omega t} \frac{d \varphi}{d x} .
\end{gathered}
$$

Note that

$$
\frac{\partial^{2} u}{\partial x^{2}}=\left[-\sum_{n=1}^{\infty} \lambda_{n} a_{n} \psi_{n}(x)\right] e^{i \omega t}
$$

These relationships can be substituted into (A-25), resulting in an equation defining the $a_{n}$ terms:

$$
a_{n}=\frac{-q_{n}}{k_{\text {eff }} \lambda_{n}-\omega^{2} m_{\text {eff }}+i \omega\left(c_{\text {visc }, \text { eff }}+\lambda_{n} c_{\text {hyst }, \text { eff }}\right)}
$$

where

$$
k_{\text {eff }}=\left(E_{\text {app }}-\frac{E_{\text {app }}^{2} d^{2}}{\mu_{0} \mu_{r}}\right) t_{\text {sens }}+2 E_{\text {sludge }}^{*} t_{\text {sludge }},
$$




$$
\begin{gathered}
m_{\text {eff }}=\rho_{\text {sens }} t_{\text {sens }}+\frac{1}{2} \rho_{f l} \sqrt{\frac{2 v}{\omega}}+2 \rho_{\text {sludge }} t_{\text {sludge }}, \\
c_{\text {visc,eff }}=2 \mu \sqrt{\frac{\omega}{v}}+\alpha \rho_{\text {sens }} t_{\text {sens }}, \\
\text { and } c_{\text {hyst }, \text { eff }}=\beta E_{\text {app }} t_{\text {sens }},
\end{gathered}
$$

as in Chapter 2. The $q_{n}$ terms can be evaluated by using the orthogonality of the eigenfunctions, such that

$$
q_{n}=\frac{E_{a p p} d t_{\text {sens }} H \int_{0}^{L} \frac{d \varphi}{d x} \psi_{n}(x) d x}{\int_{0}^{L} \phi_{n}^{2}(x) d x}=E_{a p p} d t_{\text {sens }} H \frac{2}{L} \int_{0}^{L} \frac{d \varphi}{d x} \psi_{n}(x) d x
$$

Thus, $u(x, t)$ is expressed as

$$
u(x, t)=-\sum_{n=1}^{\infty} \frac{E_{\text {app }} d t_{\text {sens }} H \frac{2}{L}\left[\int_{0}^{L} \frac{d \varphi}{d x} \psi_{n}(x) d x\right] e^{i \omega t}}{\lambda_{n}\left(k_{\text {eff }}\right)-\omega^{2}\left(m_{\text {eff }}\right)+i \omega\left[c_{\text {visc }, \text { eff }}+\lambda_{n} c_{\text {hyst }, \text { eff }}\right]} \psi_{n}(x),(A-37)
$$

the first term of which is given in equation (6) in Chapter 2.

\section{A.2.3 Voltage Induced Due to Flux Through Sensor}

In this section, the voltage induced on a hypothetical pickup coil with turns per unit length of $N$ and wrapped perfectly around the sensor is derived. To do so, the flux density in the sensor must first be determined. This is done by substituting the expression for $u(x, t)$ in (A-37) into equation (A-23) and solving for the partial derivative of $B_{x}$ with respect to $x$ : 


$$
\frac{\partial B_{x}}{\partial x}=-\frac{\mu_{o} \mu_{r}}{E_{\text {app }} d t_{\text {sens }}} u(x, t)\left(\lambda_{n}\left(k_{\text {eff }, B}\right)-\omega^{2}\left(m_{\text {eff }}\right)+i \omega\left[c_{\text {visc eff }}+\lambda_{n} c_{\text {hyst }, \text { eff }}\right]\right),
$$

where

$$
k_{\text {eff }, B}=E_{\text {app }} t_{\text {sens }}+2 E_{\text {sludge }}^{*} t_{\text {sludge }}
$$

and the infinite sum over all $n$ is implied. This can be integrated with respect to $\mathrm{x}$ (using an indefinite integral) to give

$$
B_{x}(x, t)=\sum_{n=1}^{\infty} \frac{\mu_{o} \mu_{r}}{E_{\text {app }} d t_{\text {sens }} \sqrt{\lambda_{n}}} a_{n} e^{i \omega t}\left(\lambda_{n}\left(k_{\text {eff }, B}\right)-\omega^{2}\left(m_{\text {eff }}\right)+i \omega\left[c_{\text {visceff }}+\lambda_{n} c_{\text {hyst }, \text { eff }}\right]\right) \cos \left(\sqrt{\lambda_{n}} x\right)
$$

Then, using Faraday's Law of Induction,

$$
V_{\text {sens }}(t)=-N \int_{-L}^{L} \frac{\partial\left(B_{x} d A_{\text {sens }}\right)}{\partial t} d x=-N w t_{\text {sens }} i \omega \int_{-L}^{L} B_{x} d x .
$$

This integration is straightforward and results in

$$
\begin{gathered}
V_{\text {sens }}(t)=\sum_{n=1}^{\infty} \frac{4 L_{\text {coil }} N i \omega w t_{\text {sens }} H \mu_{0} \mu_{r}}{\lambda_{n} L^{2}}\left[\int_{0}^{L} \frac{d \varphi}{d x} \sin \left(\sqrt{\lambda_{n}} x\right) d x\right] e^{i \omega t} \cdot(-1)^{n+1} . \\
\frac{\lambda_{n}\left(k_{\text {eff }, B}\right)-\omega^{2}\left(m_{\text {eff }}\right)+i \omega\left[c_{\text {visc eff }}+\lambda_{n} c_{\text {hyst }, \text { eff }}\right]}{\lambda_{n}\left(k_{\text {eff }}\right)-\omega^{2}\left(m_{\text {eff }}\right)+i \omega\left[c_{\text {visc }, \text { eff }}+\lambda_{n} c_{\text {hyst }, \text { eff }}\right]}
\end{gathered}
$$

as presented in Chapter 2.

\section{A.2.4 Voltage Induced on a Pickup Coil}

For a non-hypothetical pickup coil (i.e. one that is not wrapped perfectly around the sensor but rather has a radius $r_{c o i l}$ and length $2 L_{c o i l}$ ), the induced voltage will come from the superposition of three sources: the flux through the sensor as expressed in equation (A-42), the flux through the remainder of the pickup coil area due to the driving magnetic 
field, and the flux that is contributed by the magnetization induced in the sensor. Expressed in equation form,

$$
V_{\text {coil }}(t)=V_{\text {drive }}(t)+V_{\text {sens }}(t)+V_{\text {coil ,sens }}(t) .
$$

Using Faraday's Law of Induction, the voltage induced due to the drive field is

$$
V_{\text {drive }}(t)=-N i \omega \mu_{o} H e^{i \omega t} 2 L_{\text {coil }}\left(\pi r_{\text {coil }}^{2}-w t_{\text {sens }}\right) \text {. }
$$

The voltage due to the flux contributed by the magnetization induced in the sensor is more difficult to deduce. By definition,

$$
\mu_{o} M_{x}=B_{x}-\mu_{o} H_{x} .
$$

According to [Gou04], the Biot-Savart Law can be used to calculate the flux at a point in space due to a prismatic (rectangular in cross-section) permanent magnet as

$$
B_{\text {coil }, \text { sens }}(x, y, z, t)=\frac{\mu_{o}}{4 \pi} \int_{0}^{2 L} M_{x}\left(x_{o}, t\right)\left(d B_{x_{1}}+d B_{x_{2}}+d B_{x_{3}}+d B_{x_{4}}\right) d x_{o},
$$

where

$$
\begin{gathered}
d B_{x_{1}}=\int_{0}^{w} \frac{t_{\text {sens }} d y_{o}}{\left[\left(z-t_{\text {sens }}\right)^{2}+\left(y-y_{o}\right)^{2}+\left(x-x_{o}\right)^{2}\right]^{\frac{3}{2}}}, \\
d B_{x_{2}}=\int_{0}^{w} \frac{z d y_{o}}{\left[z^{2}+\left(y-y_{o}\right)^{2}+\left(x-x_{o}\right)^{2}\right]^{\frac{3}{2}}}, \\
d B_{x_{3}}=\int_{0}^{t_{\text {sens }}} \frac{(w-y) d z_{o}}{\left[\left(z-z_{o}\right)^{2}+(y-w)^{2}+\left(x-x_{o}\right)^{2}\right]^{\frac{3}{2}}},
\end{gathered}
$$




$$
d B_{x_{4}}=\int_{0}^{t_{\text {sens }}} \frac{y d z_{o}}{\left[\left(z-z_{o}\right)^{2}+y^{2}+\left(x-x_{o}\right)^{2}\right]^{\frac{3}{2}}},
$$

and the $\mathrm{z}$ direction is through the thickness of the sensor, while the y direction is along the width of the sensor.

Again using Faraday's Law of Induction and (A-46),

$$
V_{\text {coil sens }}(t)=-N \int_{-L_{\text {cool }}}^{L_{\text {coil }}} \frac{\partial}{\partial t}\left(\oiint_{A_{\text {coil }}}\left[\frac{\mu_{o}}{4 \pi} \int_{0}^{2 L} M_{x}\left(x_{o}, t\right)\left(d B_{x_{1}}+d B_{x_{2}}+d B_{x_{3}}+d B_{x_{4}}\right) d x_{o}\right] d A_{c o i l}\right) d x .(A-47)
$$

Clearly, the integral in (A-47) is quite complex and can be difficult to integrate even numerically. To simplify slightly, we assume that $M_{x}$ does not vary in the x-direction, such that it can be replaced with its average value $M_{x, \text { avg }}$ and brought outside the integral. Then,

$$
V_{\text {coil,sens }}(t)=-N i \omega \frac{\mu_{o}}{4 \pi} M_{x, \text { avg }}(t) S \text {, }
$$

where $S$ is the value of the resulting integral obtained via numerical integration. Using the relationship in (A-45), computing the average values of $B_{x}$ and $H_{x}$ and then using the constitutive equations to write $H_{x}$ in terms of $B_{x}$ and strain, the voltage can be written as

$$
\begin{aligned}
V_{\text {coil , sens }}(t) & =V_{\text {sens }}(t)\left(\frac{S}{8 L \pi w t_{\text {sens }}}\left(1-\frac{1}{\mu_{r}}\right)\right)+ \\
& \sum_{\mathrm{n}=1}^{\infty} N i \omega \frac{S d^{2} E_{\text {app }}{ }^{2} t_{\text {sens }} H e^{i \omega t}\left[\int_{0}^{L} \frac{d \varphi}{d x} \sin \left(\sqrt{\lambda_{n}} x\right) d x\right](-1)^{n+1}}{2 L^{2} \pi \mu_{r}\left(\lambda_{n} k_{\text {eff }}-\omega^{2}\left(m_{\text {eff }}\right)+i \omega\left[c_{\text {visc }, \text { eff }}+\lambda_{n} c_{\text {hyst }, \text { eff }}\right]\right)}
\end{aligned}
$$

Thus, the total induced voltage can be written as 


$$
\begin{aligned}
V_{\text {coil }}(t)= & V_{\text {sens }}(t)\left(1+\frac{S}{8 L \pi w t_{\text {sens }}}\left(1-\frac{1}{\mu_{r}}\right)\right)+ \\
& \sum_{\mathrm{n}=1}^{\infty} N i \omega \frac{S d^{2} E_{\text {app }}{ }^{2} t_{\text {sens }} H e^{i \omega t}\left[\int_{0}^{L} \frac{d \varphi}{d x} \sin \left(\sqrt{\lambda_{n}} x\right) d x\right](-1)^{n+1}}{2 L^{2} \pi \mu_{r}\left(\lambda_{n} k_{\text {eff }}-\omega^{2}\left(m_{\text {eff }}\right)+i \omega\left[c_{\text {visc ,eff }}+\lambda_{n} c_{\text {hyst }, \text { eff }}\right]\right)}-,(A-50) \\
& N i \omega \mu_{o} H e^{i \omega t} 2 L_{\text {coil }}\left(\pi r_{\text {coil }}{ }^{2}-w t_{\text {sens }}\right)
\end{aligned}
$$

just as in equation (14) of Chapter 2.

\section{A.2.5 Shape Function}

For the purposes of this work, the shape function is determined numerically for a given set of sensor dimensions and permeability. To increase the ease of use in the analytical model, the numerical results are fit with a function of the form

$$
\varphi=\frac{A \cosh (B)-A \cosh \left(B \frac{x}{L}\right)}{\cosh (B)-1} .
$$

Examples of the shape function for two different materials are given in Fig. A-2.
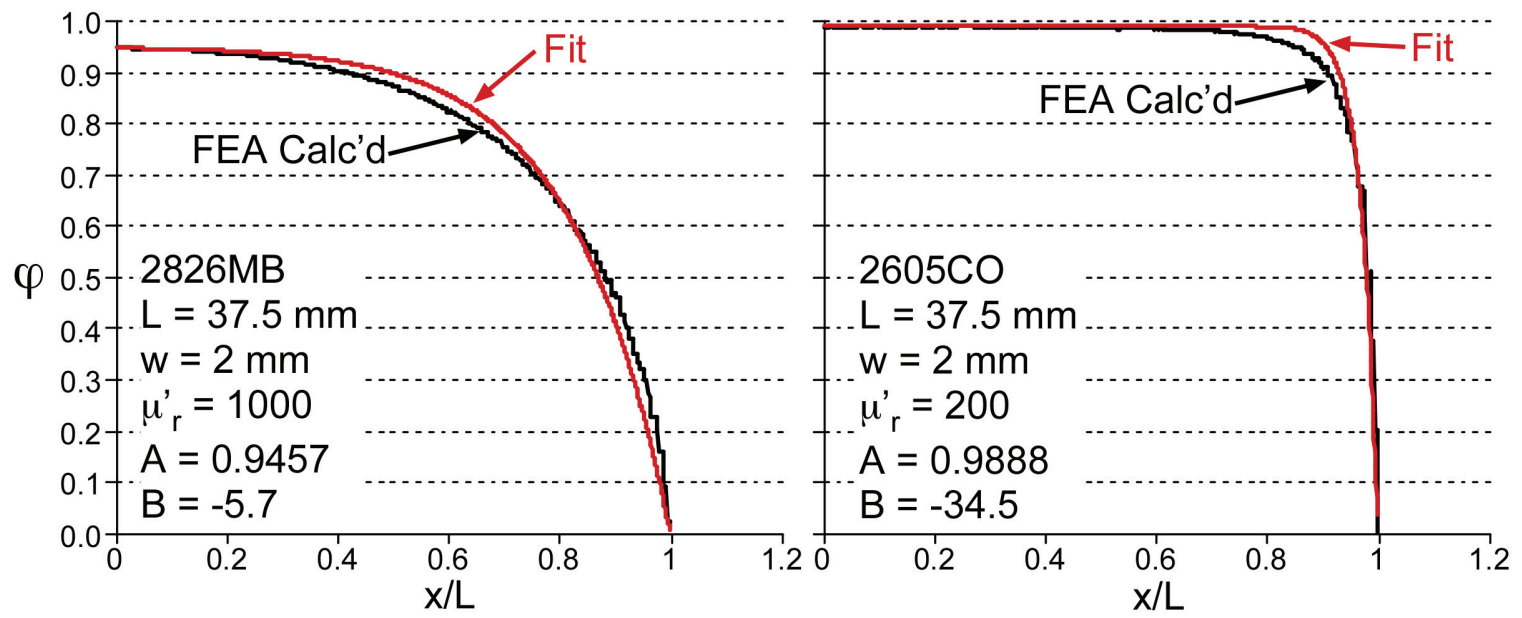

Fig. A-2: Examples of shape functions for two different materials. 2826MB (left) has a higher permeability than $2605 \mathrm{CO}$ (right) and is less easily penetrated by the magnetic field. 
With this form of shape function, the value of the integral used throughout the previous derivation is

$$
\int_{0}^{L} \frac{d \varphi}{d x} \sin \left(\sqrt{\lambda_{n}} x\right) d x=\frac{4 A B^{2}\left(1+e^{2 B}\right)}{\left(1+e^{2 B}-2 e^{B}\right)\left((2 n-1)^{2} \pi^{2}+4 B^{2}\right)}(-1)^{n} .
$$

Table A-1 lists selected values of $\mathrm{A}$ and $\mathrm{B}$ that were found for the materials and geometries studied here. The complete list is given in the MatLab code in section A.2.6.

Table A-1: Selected values of parameters A and B used in analytical model

\begin{tabular}{|c|c|c|c|c|c|}
\hline Alloy (as-cast) & $\boldsymbol{\mu}_{\boldsymbol{r}}^{\prime}$ & Length $(\mathbf{m m})$ & Width $(\mathbf{m m})$ & $\mathbf{A}$ & B \\
\hline $2826 \mathrm{MB}$ & 1000 & 15 & 0.5 & 0.86198 & -4.2 \\
\hline $2826 \mathrm{MB}$ & 1000 & 15 & 3 & 0.62175 & -3.7 \\
\hline $2826 \mathrm{MB}$ & 1000 & 37.5 & 0.5 & 0.98165 & -8.4 \\
\hline $2826 \mathrm{MB}$ & 1000 & 37.5 & 3 & 0.92506 & -5.3 \\
\hline $2605 \mathrm{SA} 1$ & 2500 & 15 & 0.5 & 0.70373 & -3.2 \\
\hline $2605 \mathrm{SA} 1$ & 2500 & 15 & 3 & 0.41480 & -3.2 \\
\hline $2605 \mathrm{SA} 1$ & 2500 & 37.5 & 0.5 & 0.95853 & -5.6 \\
\hline $2605 \mathrm{SA} 1$ & 2500 & 37.5 & 3 & 0.81555 & -3.9 \\
\hline $2605 \mathrm{CO}$ & 200 & 15 & 0.5 & 0.99216 & -23.9 \\
\hline $2605 \mathrm{CO}$ & 200 & 15 & 3 & 0.97457 & -13.1 \\
\hline $2605 \mathrm{CO}$ & 200 & 37.5 & 0.5 & 0.99078 & -60.8 \\
\hline $2605 \mathrm{CO}$ & 200 & 37.5 & 3 & 0.98653 & -32.4 \\
\hline
\end{tabular}

\section{A.2.6 MatLab Implementation of Analytical Model}

The following script can be run in MatLab to determine important results from the analytical model for various sensor geometries and loading conditions:

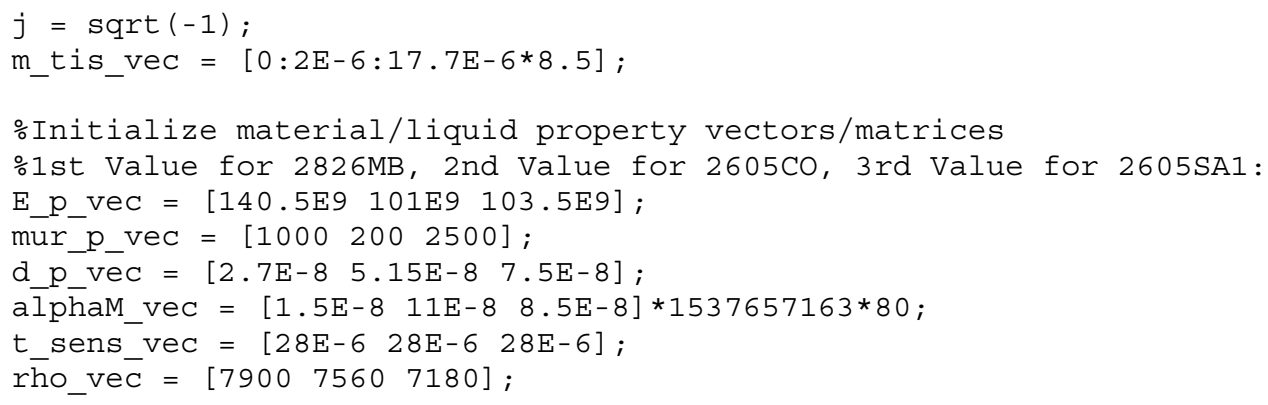


$\div$ A, B are 5X5X3 matrices (Length, Width, Material) defining shape functions $\%$ (fit by cosh)

$\%(\mathrm{~L}=15 \mathrm{~mm}, 20 \mathrm{~mm}, 25 \mathrm{~mm}, 30 \mathrm{~mm}, 37.5 \mathrm{~mm} ; \mathrm{w}=0.5 \mathrm{~mm}, 1 \mathrm{~mm}, 2 \mathrm{~mm}, 3 \mathrm{~mm}, 6 \mathrm{~mm}$;

$\div \mathrm{M}=2826 \mathrm{MB}, 2605 \mathrm{CO}, 2605 \mathrm{SA})$

A_mat $(:,:, 1)=\left[\begin{array}{lllllll}0.861982 & 0.775301 & 0.678467 & 0.621749 & 0.539103 ; & 0.926032 \ldots\end{array} \ldots\right.$ $\begin{array}{lllllllllll}0.86766 & 0.790946 & 0.740064 & 0.659777 ; & 0.956572 & 0.918831 & 0.861212 & 0.820508 & \ldots\end{array}$

$\begin{array}{llllllll}0.750722 ; & 0.971615 & 0.947628 & 0.906311 & 0.874931 & 0.816810 ; & 0.981649 & \ldots\end{array}$

$0.9698410 .945715 \quad 0.925063 \quad 0.884295] ;$

B_mat $(:,:, 1)=\left[\begin{array}{lllllllllllllllllll}-4.2 & -3.8 & -3.7 & -3.7 & -3.9 ; & -5.1 & -4.4 & -4.1 & -4 & -4 ; & -6 & -5 & \ldots\end{array}\right.$

$\left.\begin{array}{llllllllllllll}-4.5 & -4.3 & -4.2 ; & -6.9 & -5.7 & -5 & -4.7 & -4.4 ; & -8.4 & -6.8 & -5.7 & -5.3 & -4.9\end{array}\right] ;$

A_mat $(:,:, 2)=\left[\begin{array}{llllll}0.992157 & 0.985853 & 0.979561 & 0.974569 & 0.961858 ; & 0.990808 \ldots\end{array} \ldots\right.$ $0.9846290 .9860190 .981386 \quad 0.975323 ; \quad 0.982174 \quad 0.992522 \quad 0.986410 \quad 0.984951 \quad \ldots$

$\begin{array}{lllllllll}0.982071 ; & 0.987298 & 0.989467 & 0.987956 & 0.987997 & 0.985493 ; & 0.990780 & \ldots\end{array}$

$0.990245 \quad 0.988784 \quad 0.986529 \quad 0.988288]$;

B_mat $(:,:, 2)=\left[\begin{array}{lllllllllll}-23.9 & -18.1 & -14.9 & -13.1 & -12.2 ; & -40.8 & -27 & -23.8 & -21.1 & \ldots\end{array}\right.$

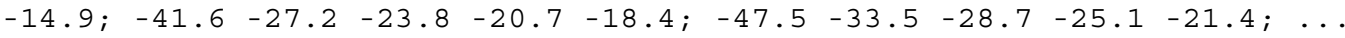

$-60.8-45.4-34.5-32.4-27.2] ;$

A_mat $(:,:, 3)=\left[\begin{array}{llllll}0.703729 & 0.582416 & 0.471125 & 0.414797 & 0.340806 ; & 0.817818 \ldots\end{array}\right.$

$\begin{array}{llllllllll}0.713872 & 0.603307 & 0.541265 & 0.454261 ; & 0.885921 & 0.803842 & 0.704417 & 0.644701 & \ldots\end{array}$

$\begin{array}{lllllllll}0.555145 ; & 0.926264 & 0.864133 & 0.780334 & 0.726591 & 0.640037 ; & 0.958527 & \ldots\end{array}$

$0.919882 \quad 0.858971 \quad 0.815547 \quad 0.741127]$;

B_mat $(:,:, 3)=\left[\begin{array}{llllllllllllll}-3.2 & -3 & -3.1 & -3.2 & -3.5 ; & -3.7 & -3.4 & -3.3 & -3.3 & -3.4 ; & -4.2 & \ldots\end{array}\right.$

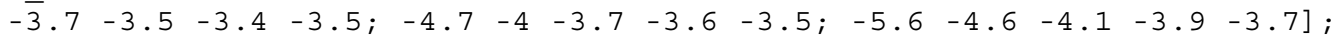

\%Load properties Biofilm, Paraffin 1, Paraffin 2

$\mathrm{E}$ tis_vec $=\left[\begin{array}{lll}8 \mathrm{E} 3 & 30 \mathrm{E} 6 & 2 \mathrm{E} 9\end{array}\right]$;

d_fac_vec $=\left[\begin{array}{lll}10 / 8 & 2.5 \mathrm{E} 6 / 30 \mathrm{E} 6 & 5 \mathrm{E} 8 / 2 \mathrm{E} 9\end{array}\right] ;$

rho_t_vec $=\left[\begin{array}{lll}1200 & 930 & 930\end{array}\right]$;

$\%$ Fluid properties [air, DI water, 12CP]

nu_vec $=[1.008 \mathrm{E}-61.008 \mathrm{E}-61.011 \mathrm{E}-5]$;

mu_vec $=\left[\begin{array}{lll}0 & 1.008 \mathrm{E}-3 & 12.385 \mathrm{E}-3\end{array}\right]$;

rho_f_vec $=\left[\begin{array}{lll}0 & 1000 & 1225\end{array}\right]$;

\%Geometry vectors ( $L$ is half the sensor length!)

L_vec $=\left[\begin{array}{lllll}0.0075 & 0.01 & 0.0125 & 0.015 & 0.01875\end{array}\right] ;$

$\mathrm{w}-\mathrm{vec}=\left[\begin{array}{lllll}.5 \mathrm{E}-3 & 1 \mathrm{E}-3 & 2 \mathrm{E}-3 & 3 \mathrm{E}-3 & 6 \mathrm{E}-3\end{array}\right] ;$

$\because$ Constants

$\mathrm{nu} \mathrm{p}=0.22$;

$\mathrm{mu} \overline{0}=1.256 \mathrm{E}-6 ;$

$\mathrm{N} t=27$; $\%$ Total number of turns!

L_coil $=0.022 ; \%$ Half-length of pickup coil, to normalize sensor voltage

r_coil $=0.0515$;

N_p_L $=$ N_t/ $(2 *$ L_coil $) ; \%$ Turns $/$ meter

HaC $=19 . \overline{5} ;$ oMagnetic field amplitude in $\mathrm{A} / \mathrm{m}$

OPreallocate for speed:

amp = $\quad$ zeros $\left(3\right.$, length $\left(L_{-}\right.$vec $)$, length $\left(w_{-}\right.$vec $)$, length $($E_tis_vec $), \ldots$

length(nu_vec), length (m_tis_vec), 31);

omega_0_est $=$ zeros $\left(3\right.$, length $\left(L_{-}\right.$vec $)$, length $\left(w \_v e c\right)$, length $\left(E \_t i s \_v e c\right), \ldots$ length $\left.\overline{\mathrm{n} u \_v e c}\right)$, length (m_tis_vec $) \overline{)}$;

amp_r $=$ zeros $\left(3\right.$, length $\left(L \_v e c\right)$, length $\left(w \_v e c\right)$, length $\left(E \_t i s \_v e c\right), \ldots$

length (nu_vec), length (m_tis_vec), 181); 


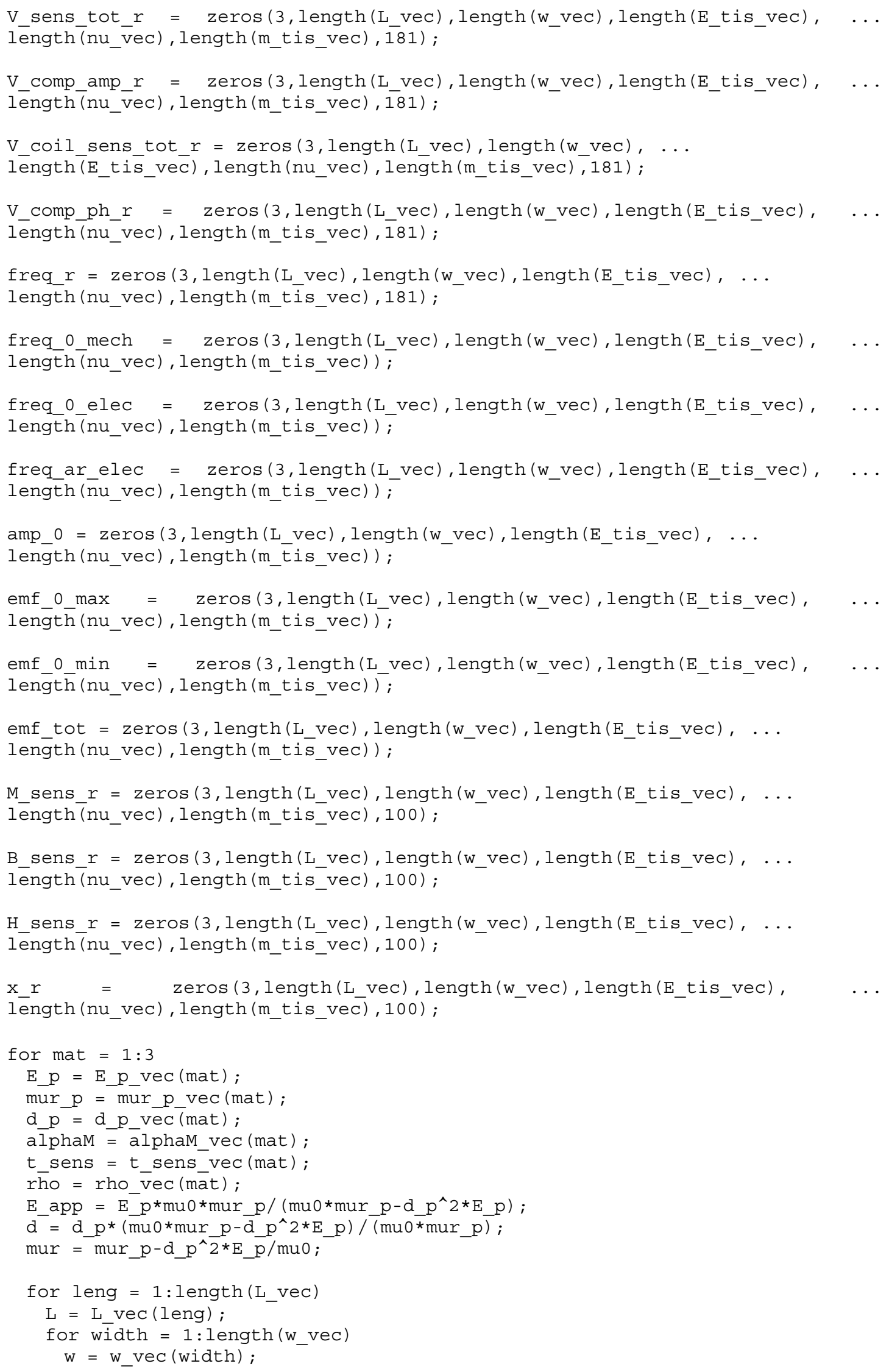




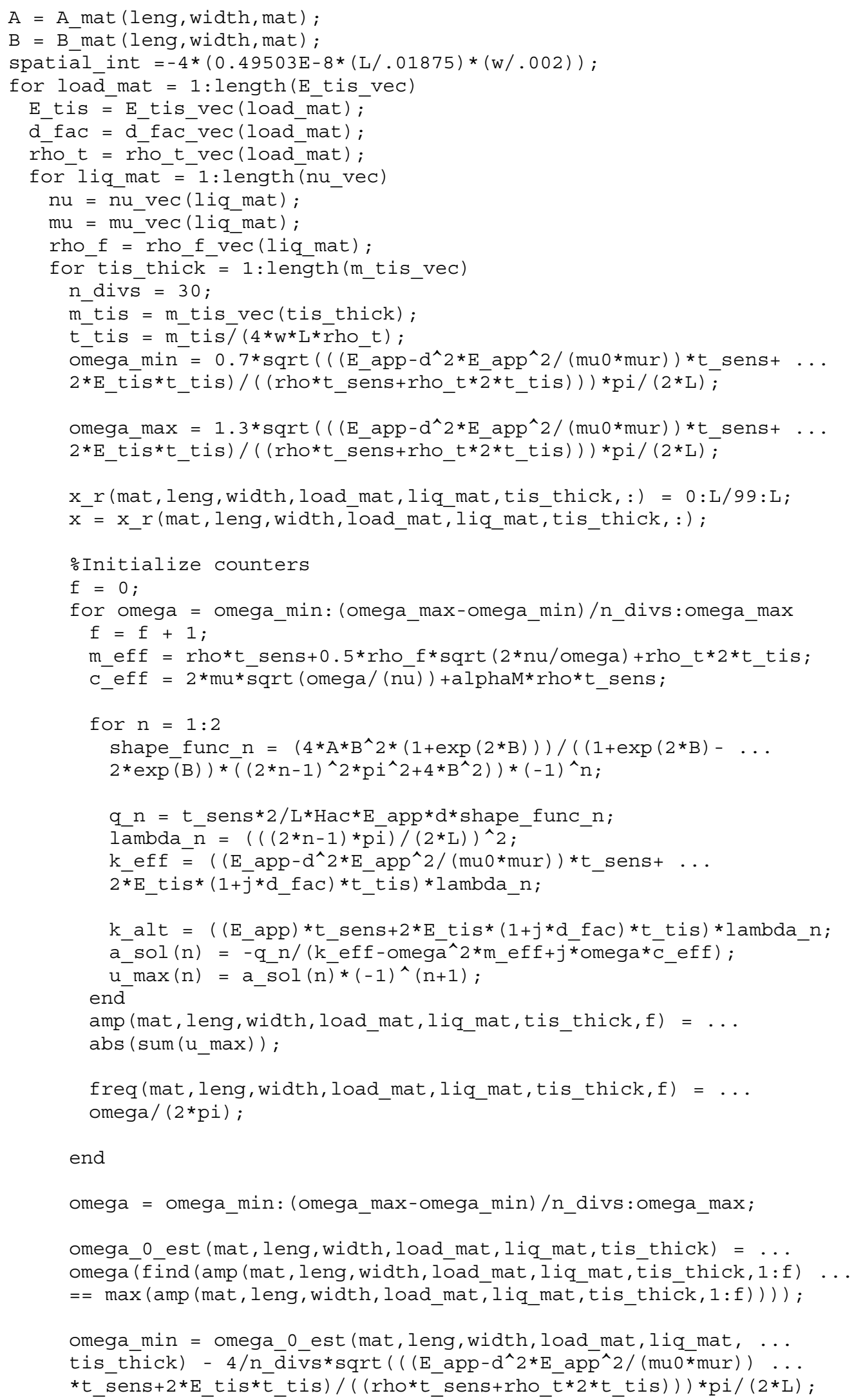




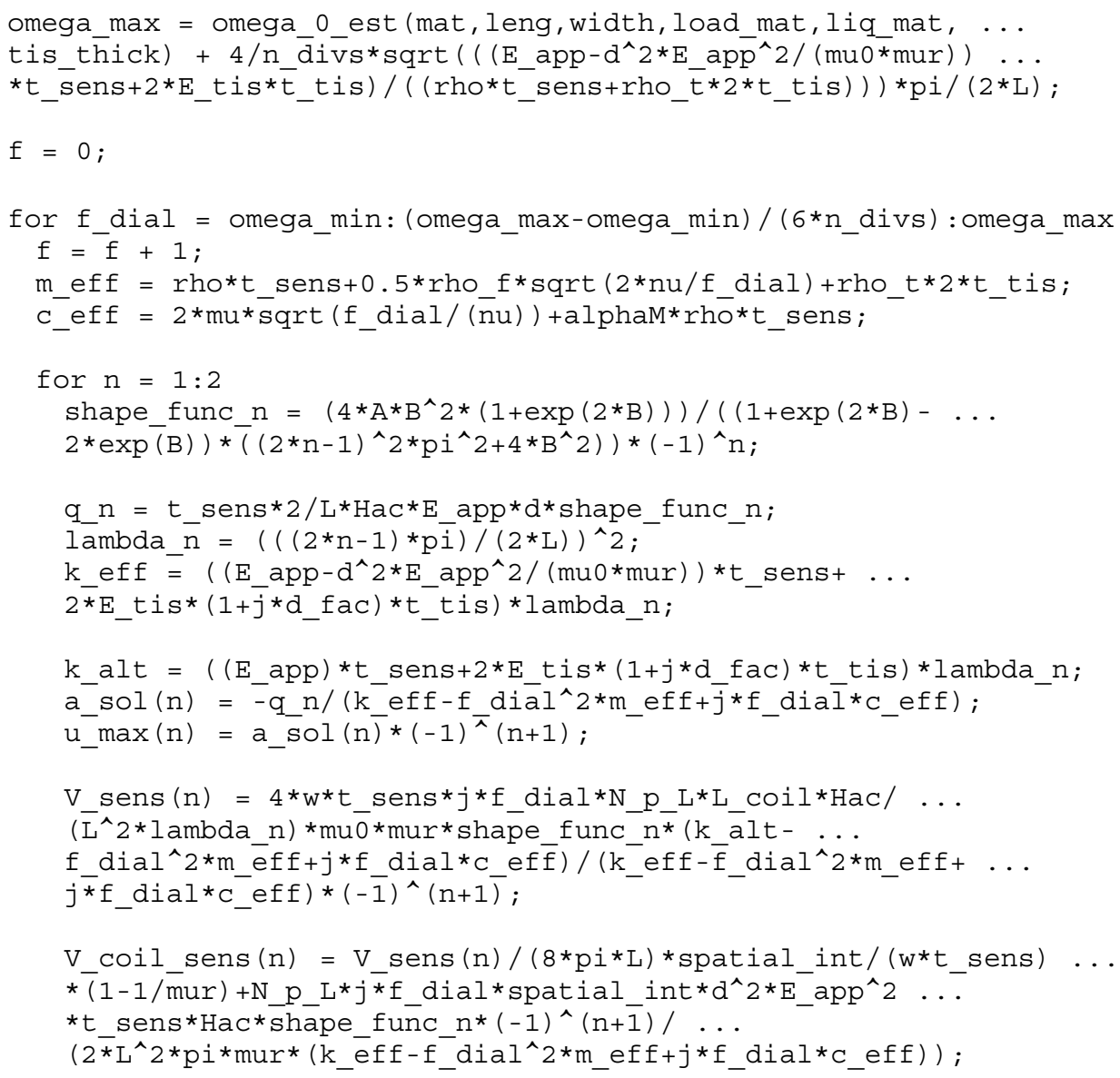

end

omega_0=omega_min: (omega_max-omega_min) / (6*n_divs) :omega_max; 


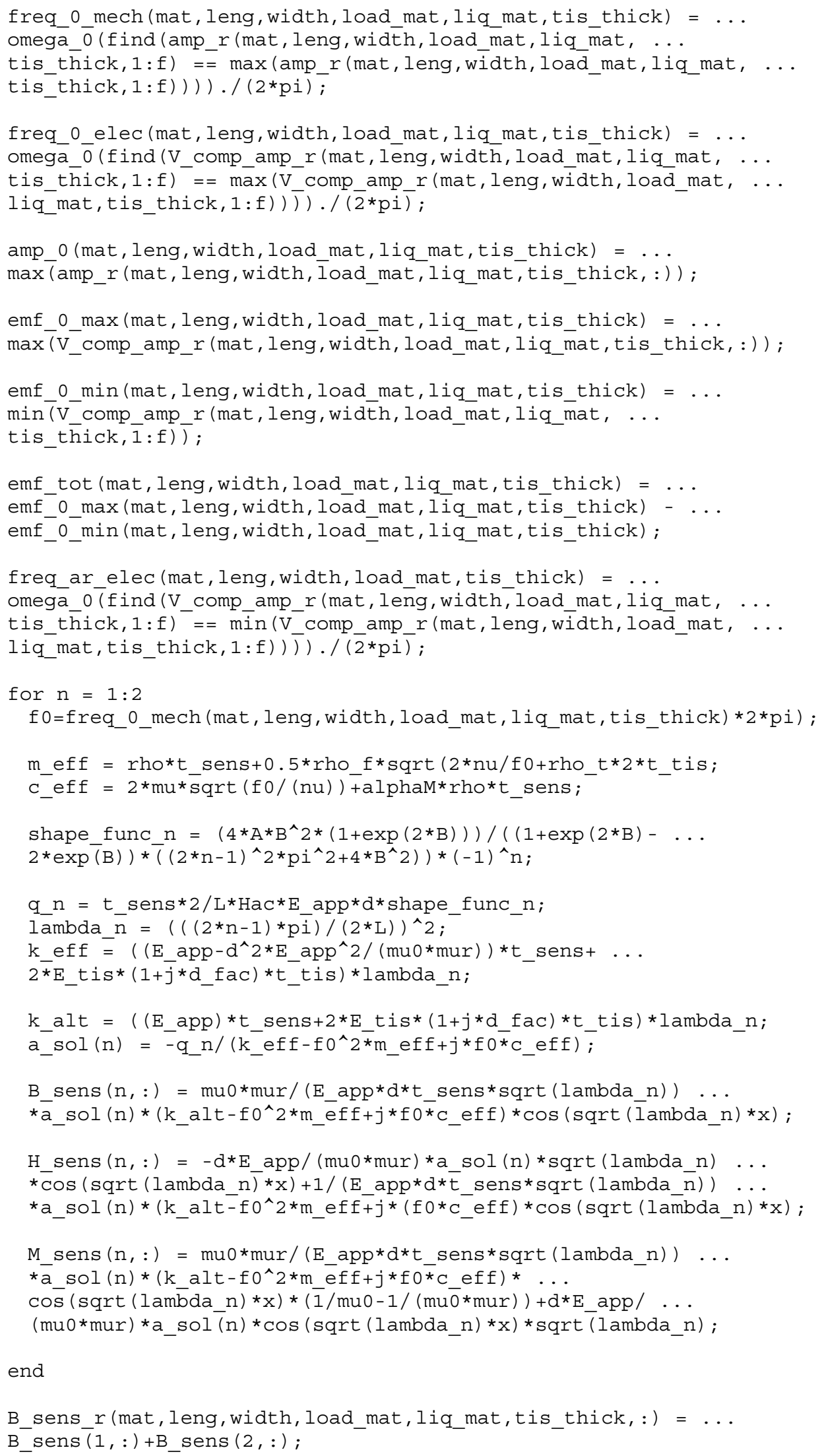




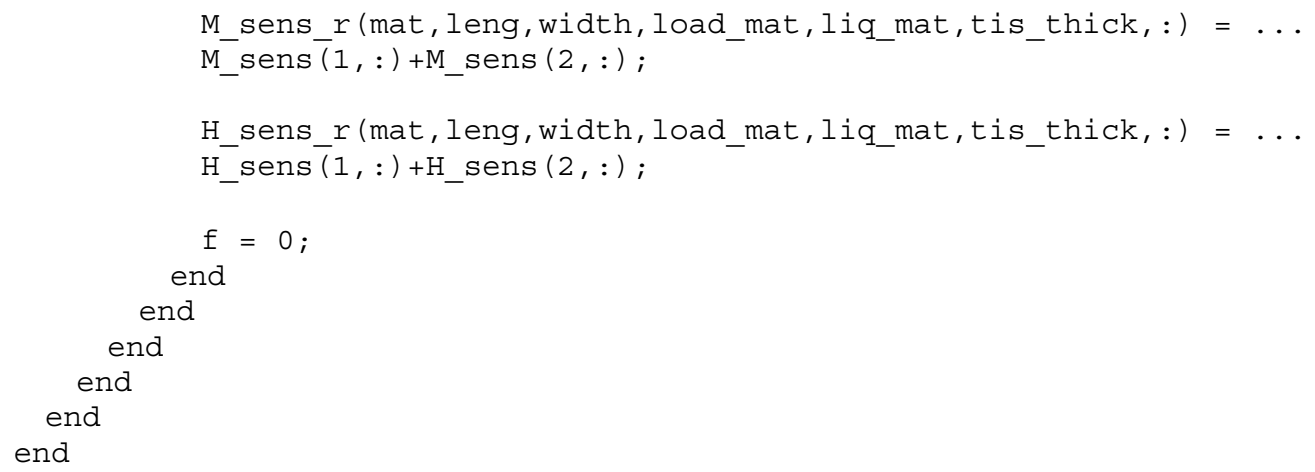

Important results are listed in Table A-3 (in section A.4), or presented in the main chapters of this work.

\section{A.3 Magnetomechanically-Coupled Finite Element Model}

The constitutive equations (A-1) and (A-2) can also be implemented in a multiphysics finite element software package so that more complex resonator geometries can be simulated, as discussed in section 2.2.3. This section describes the implementation of the equations into COMSOL Multiphysics (version 3.4), along with other modifications required for a fully magnetomechanically-coupled simulation. Also, helpful hints for scripting a frequency-response analysis, post-processing results, and geometry modeling are included.

\section{A.3.1 Constitutive Equation Rearrangement}

Because most finite element models use strain (or more precisely, derivatives of displacements) and flux density (or more precisely, magnetic potential) as the parameters that are directly solved for and calculate other parameters in terms of strain and flux density, the constitutive equations are most easily implemented by rearranging (A-1) and 
(A-2) so that stress and the magnetic field are written in terms of strain and flux density.

First, we abbreviate by writing (A-1) and (A-2) as

$$
\begin{gathered}
\vec{\varepsilon}=\left[C^{\prime}\right] \vec{\sigma}+\left[d^{\prime}\right] \vec{H} \\
\text { and } \vec{B}=\left[d^{\prime}\right]^{T} \vec{\sigma}+\left[\mu_{o} \mu_{r}^{\prime}\right] \vec{H} .
\end{gathered}
$$

Then (A-53) and (A-54) can be rearranged:

$$
\vec{\sigma}=[C] \mathcal{E}-[C]\left[d^{\prime}\right]\left[\mu_{o} \mu_{r}^{\prime}\right]^{-1} \vec{B}
$$

and $\vec{H}=-\left[\mu_{o} \mu_{r}^{\prime}\right]^{-1}\left[d^{\prime}\right]^{T}[C] \vec{\varepsilon}+\left(\left[\mu_{o} \mu_{r}^{\prime}\right]^{-1}+\left[\mu_{o} \mu_{r}^{\prime}\right]^{-1}\left[d^{\prime}\right]^{T}[C]\left[d^{\prime}\right]\left[\mu_{o} \mu_{r}^{\prime}\right]^{-1}\right) \vec{B}$

where

$$
[C]=\left([I]-\left[C^{\prime}\right]^{-1}\left[d^{\prime}\right]\left[\mu_{o} \mu_{r}^{\prime}\right]^{-1}\left[d^{\prime}\right]^{T}\right)^{-1}\left[C^{\prime}\right]^{-1} .
$$

It can be seen that (A-55) and (A-56) are of the same form as equations (1) and (2) in section 2.2.1, assuming the terms contributed by $\left[\mu_{o} \mu_{r}^{\prime}\right]^{-1}\left[d^{\prime}\right]^{T}[C]\left[d^{\prime}\right]\left[\mu_{o} \mu_{r}^{\prime}\right]^{-1}$ can be neglected in (A-56). These terms are often approximately $10 \%$ as large as the $\left[\mu_{o} \mu_{r}^{\prime}\right]^{-1}$ terms in (A-56); however, the terms will be kept going forward. Furthermore, (A-55) and (A-56) will be abbreviated as

$$
\begin{array}{r}
\vec{\sigma}=[s e] \mathcal{E}+[s b] \vec{B} \\
\text { and } \vec{H}=[h e] \vec{\varepsilon}+[h b] \vec{B}
\end{array}
$$

\section{A.3.2 COMSOL Multiphysics Model Development}

As stated previously, Version 3.4 of COMSOL Multiphysics is used for the model developed in this work. Upon initializing the program and viewing the model navigator window, the space dimension should be changed to "3D" and the "Multiphysics" button 
should be pushed. In the "AC/DC Module", choose "Quasi-Statics, Magnetic", then "Induction Currents", then "Time-Harmonic Analysis". The element should be chosen as "Vector - Linear", although higher order elements could be used at the cost of computational speed. This domain should then be added by pressing the "Add" button. Under the "Structural Mechanics Module", choose "Solid, Stress-Strain" and then "Frequency Response Analysis". The element should be chosen as "LaGrange - Linear", although again higher order elements could be used at the cost of computational speed. The ruling application mode should be set to "Solid, Stress-Strain". The "OK" button can then be pressed to open a new model.

\section{A.3.2.1 Geometry Modeling}

The transmit coil, pickup coil, and sensor subdomains should be modeled while taking advantage of appropriate symmetry planes to reduce the size of the model. The COMSOL "Draw" menu can be utilized for simple shapes and composite (Boolean) objects. It should be noted that, when creating composite objects that contain or are near fine features, the repair option should be turned on and set to a "small" tolerance (e.g. 1E8) to ensure that surfaces are appropriately aligned after the composite object creation.

For more complex part geometries, it is often useful to import an IGES file that has been created with a dedicated solid modeling program (e.g. Pro/Engineer or Solid Works). When imported parts have small features, ensure that the import repair tolerances are appropriately small. As noted in the main chapters of this work (particularly Chapters 4 and 5), some sensor geometries must be curved to fit along the inner stent wall. It is often necessary to model the sensor in this curved shape in order to accurately predict the 
resulting changes to mode shapes, resonant frequencies, and signal amplitudes. Modeling complex sensors with a curved shape is not simple, but can be accomplished with the following procedure (using Pro/Engineer; other solid modeling programs may require a different procedure): First, model the sensor geometry in a planar state. Then, project the layout of the sensor pattern onto a datum plane. Generate a curved surface (with the desired diameter) above this datum plane, and "wrap" the layout from the datum plane onto the curved surface. Trim the curved surface using the wrapped layout. The trimmed surface can then be thickened to result in the final curved sensor geometry. The planar geometry should be cut away and an appropriate datum coordinate system added so that the curved geometry can be exported as an IGES file and imported into COMSOL. As a final note for geometry modeling, it was found that modeling the coils with a cylinder and the sensor with curved geometry (as just described) often resulted in meshing errors. These errors can be avoided by modeling the coils with a faceted "curved" surface (by extruding a 2D sketch) rather than by using the cylinder tool in COMSOL.

\section{A.3.2.2 Constants}

Under the "Options" menu, open "Constants". Here material constants and the entries for the constitutive matrices ([he], $[h b],[s b]$ and $[s e]$ ) can be entered and assigned to variable names. Table A-2 lists the material constants that were found to result in good agreement with the experimentally obtained signal from ribbon sensors of the size and material described (and subsequently used for the results presented in the main chapters of this report). The assigned expressions for the constitutive matrices follow. 
Table A-2: Material constants fitted to experimental results for various Metglas ${ }^{\mathrm{TM}}$ alloys

\begin{tabular}{|c|c|c|c|c|c|c|c|}
\hline Alloy & Processing & $\begin{array}{c}\text { Density } \\
{\left[\mathrm{kg} / \mathrm{m}^{3}\right]} \\
\rho \\
\text { (rho_d) }\end{array}$ & $\begin{array}{c}\text { Relative } \\
\text { Permeability } \\
\mu_{r}^{\prime} \\
(\mathrm{mur})\end{array}$ & $\begin{array}{c}\text { Young's } \\
\text { Mod. } \\
\text { [Pa] } \\
E^{\prime} \\
\left(\mathbf{E} \_p\right)\end{array}$ & $\begin{array}{c}\text { Magneto- } \\
\text { strictivity } \\
\text { d' [m/A] } \\
\text { (d_p) }\end{array}$ & $\begin{array}{c}\text { Mass- } \\
\text { Proportional } \\
\text { Damping } \\
\text { Factor }[1 / \mathrm{s}] \\
\alpha \\
(\mathbf{k})\end{array}$ & $\begin{array}{c}\text { Poisson's } \\
\text { Ratio } \\
v_{s} \\
\text { (nu) }\end{array}$ \\
\hline $2826 \mathrm{MB}$ & EDM, As-Cast & 7900 & 1000 & $140.5 \mathrm{E} 9$ & $2.7 \mathrm{E}-8$ & 1845 & 0.22 \\
\hline $2605 \mathrm{CO}$ & EDM, As-Cast & 7560 & 200 & 101E9 & $5.15 \mathrm{E}-8$ & 13531 & 0.22 \\
\hline 2605SA1 & EDM, As-Cast & 7180 & 2500 & $103.5 \mathrm{E} 9$ & $7.5 \mathrm{E}-8$ & 10456 & 0.22 \\
\hline $2605 \mathrm{SA} 1$ & PCM, As-Cast & 7180 & 2500 & 148E9 & $2.3 \mathrm{E}-8$ & 7381 & 0.22 \\
\hline $2605 \mathrm{SA} 1$ & $\begin{array}{c}\text { EDM, } \\
\text { Transverse } \\
\text { Annealed }\end{array}$ & 7180 & 1250 & $37.5 \mathrm{E} 9$ & $29.5 \mathrm{E}-8$ & 46129 & 0.22 \\
\hline 2605SA1 & $\begin{array}{c}\text { PCM, } \\
\text { Transverse } \\
\text { Annealed } \\
\end{array}$ & 7180 & 1250 & $50.5 \mathrm{E} 9$ & $19.5 \mathrm{E}-8$ & 31368 & 0.22 \\
\hline 2605SA1 & $\begin{array}{c}\text { PCM, } \\
\text { Transverse } \\
\text { Annealed* }\end{array}$ & 7180 & 1250 & $66.5 \mathrm{E} 9$ & $10.75 \mathrm{E}-8$ & 55970 & 0.22 \\
\hline
\end{tabular}

The headings of the columns denote the name of the material parameter, followed by the units (in brackets), followed by the symbol used in this work, followed by the variable name assigned in COMSOL (in parentheses). All parameters fit to experimental results from $30 \mathrm{~mm} \times 2 \mathrm{~mm}$ ribbon sensors unless otherwise noted. *Parameters fit to experimental results from a $15 \mathrm{~mm} \times 1 \mathrm{~mm}$ ribbon sensor.

$$
\begin{gathered}
{[h b]=\left[\begin{array}{cccccc}
h b_{11} & h b_{12} & h b_{12} & 0 & 0 & 0 \\
h b_{12} & h b_{11} & h b_{12} & 0 & 0 & 0 \\
h b_{12} & h b_{12} & h b_{11} & 0 & 0 & 0 \\
0 & 0 & 0 & h b_{11} & 0 & 0 \\
0 & 0 & 0 & 0 & h b_{11} & 0 \\
0 & 0 & 0 & 0 & 0 & h b_{11}
\end{array}\right] \text { (A-60) }} \\
h b_{11}=-\frac{d^{\prime 2} E^{\prime}+2 \mu_{o} \mu_{r}^{\prime} v_{s}^{2}+\mu_{o} \mu_{r}^{\prime} v_{s}-\mu_{o} \mu_{r}^{\prime}}{d^{\prime 4} E^{\prime 2}-2 d^{\prime 2} E^{\prime} \mu_{o} \mu_{r}^{\prime}+d^{\prime 2} E^{\prime} \mu_{o} \mu_{r}^{\prime} v_{s}-2\left(\mu_{o} \mu_{r}^{\prime} v_{s}\right)^{2}+\left(\mu_{o} \mu_{r}^{\prime}\right)^{2}-\left(\mu_{o} \mu_{r}^{\prime}\right)^{2} v_{s}}(A-61) \\
h b_{12}=\frac{d^{\prime 2} E^{\prime} v_{s}}{d^{\prime 4} E^{\prime 2}-2 d^{\prime 2} E^{\prime} \mu_{o} \mu_{r}^{\prime}+d^{\prime 2} E^{\prime} \mu_{o} \mu_{r}^{\prime} v_{s}-2\left(\mu_{o} \mu_{r}^{\prime} v_{s}\right)^{2}+\left(\mu_{o} \mu_{r}^{\prime}\right)^{2}-\left(\mu_{o} \mu_{r}^{\prime}\right)^{2} v_{s}}(A-62)
\end{gathered}
$$




$$
\begin{aligned}
& {[h e]=\left[\begin{array}{cccccc}
h e_{11} & h e_{12} & h e_{12} & 0 & 0 & 0 \\
h e_{12} & h e_{11} & h e_{12} & 0 & 0 & 0 \\
h e_{12} & h e_{12} & h e_{11} & 0 & 0 & 0 \\
0 & 0 & 0 & 0 & 0 & 0 \\
0 & 0 & 0 & 0 & 0 & 0 \\
0 & 0 & 0 & 0 & 0 & 0
\end{array}\right]} \\
& h e_{11}=\frac{d^{\prime} E^{\prime}\left(h b_{11}\left(v_{s}-1\right)-2 h b_{12} v_{s}\right)}{\left(1+v_{s}\right)\left(1-2 v_{s}\right)} \\
& h e_{12}=-\frac{d^{\prime} E^{\prime}\left(h b_{11} v_{s}+h b_{12}\right)}{\left(1+v_{s}\right)\left(1-2 v_{s}\right)} \\
& {[s b]=[h e]} \\
& {[s e]=\left[\begin{array}{cccccc}
s e_{11} & s e_{12} & s e_{12} & 0 & 0 & 0 \\
s e_{12} & s e_{11} & s e_{12} & 0 & 0 & 0 \\
s e_{12} & s e_{12} & s e_{11} & 0 & 0 & 0 \\
0 & 0 & 0 & s e_{44} & 0 & 0 \\
0 & 0 & 0 & 0 & s e_{44} & 0 \\
0 & 0 & 0 & 0 & 0 & s e_{44}
\end{array}\right]} \\
& s e_{11}=\frac{E^{\prime}\left(h e_{11} d^{\prime}\left(v_{s}-1\right)-2 h e_{12} d^{\prime} v_{s}-v_{s}+1\right)}{\left(1+v_{s}\right)\left(1-2 v_{s}\right)} \\
& s e_{12}=\frac{E^{\prime}\left(v_{s}-h e_{11} d^{\prime} v_{s}-h e_{12} d^{\prime}\right)}{\left(1+v_{s}\right)\left(1-2 v_{s}\right)} \\
& s e_{44}=\frac{E^{\prime}}{\left(1+v_{s}\right)}
\end{aligned}
$$

Note that the material properties seem to be a function of processing conditions as well as feature size. This may explain some of the poor fit between predicted response and measured response for the more complicated geometries described in Chapters 4 and 5 . Further development of a parameter database for various processing conditions and feature sizes should result in improved predictive modeling accuracy. 


\section{A.3.2.3 Establishing Model Physics}

After modeling the appropriate geometry and establishing the material parameters and constitutive equation coefficients, the physics settings for the model and each subdomain should be established. Under the "Physics" menu, select "Scalar Variables" and set the expression for "nu_emqa" and "freq_smsld" to "freq", which will be the global variable name for the cyclical frequency of the analysis. Under the "Physics" menu, select "Subdomain Settings". While in the "Solid, Stress-Strain" domain (which can be chosen using the "Multiphysics" menu), ensure that only the magnetoelastic subdomain is active. Under the "Material" tab, set "E" to "E_p", "v" to "nu", and " $\rho$ " to "rho_d". Under the "Damping" tab, set the damping model to "Rayleigh", and set " $\alpha_{\mathrm{dm}}$ " to " $\mathrm{k}$ " and " $\beta_{\mathrm{dk}}$ " to " 0 ". Note that the stiffness damping proportionality is set to zero as this helps computational speed without any noticeable effects on the frequency response of ribbon sensors. However, it may be found in the future that a non-zero value of the stiffness damping proportionality may capture important effects in the frequency response. While in the "Induction Currents" domain, ensure all subdomains are active and assign the appropriate permeability to each subdomain. In the magnetoelastic subdomain, this involves setting " $\mu_{\mathrm{r}}$ " to "mur".

The next step involves setting the governing equations for the magnetoelastic subdomain. Under the "Physics" menu, select "Equation System" and then "Subdomain Settings". Select the magnetoelastic subdomain, and navigate to the "Variables" tab. In this tab, locate the equations that should be coupled (as suggested by the constitutive equations) and rewrite them to match the constitutive equations. For example:

$$
\begin{gathered}
H x_{-} \text {emqa }=h e 11 * e x_{-} \text {smsld }+h e 12 * e y_{-} \text {smsld }+h e 12 * e z_{-} \text {smsld } \\
+h b 11 * B x_{-} \text {emqa }+h b 12 * B y_{-} \text {emqa }+h b 13 * B z_{-} \text {emqa }
\end{gathered}
$$


and so on for Hy_emqa and $H_{z} \_$emqa;

$$
\begin{aligned}
& \text { sx_smsld }=s e 11 * e x_{-} \text {smsld }+ \text { se } 12 * e y \_s m s l d+s e 12 * e z \_s m s l d \\
& +s b 11 * B x \_e m q a+s b 12 * B y \_e m q a+s b 13 * B z \text { emqa }
\end{aligned}
$$

and so on for sy_smsld, sz_smsld, sxy_smsld, syz_smsld, and sxz_smsld; and

$$
\begin{aligned}
& s x_{-} t_{-} \text {smsld }=s e 11 * e x \_t \text { _smsld }+ \text { se } 12 * e y_{-} t_{-} \text {smsld }+\operatorname{se} 12 * e z_{-} t_{-} \text {smsld } \\
& +j * \text { omega } *\left(s b 11^{*} B x_{-} \text {emqa }+ \text { sb12*By_emqa }+s b 13 * B z_{-} \text {emqa }\right)
\end{aligned}
$$

and so on for $s y \_t \_s m s l d, s z \_t \_s m s l d$, sxy_t_smsld, syz_t_smsld, and sxz_t_smsld. In (A71) through (A-73), the coefficients he11, he12, etc. are already defined in the model constants as described in section A.3.2.2. The suffixes_emqa and smsld denote the applicability of the variable to the "Induction Currents" and "Solid, Stress-Strain" domains, respectively. Finally, the $\_t$ descriptor in (A-73) denotes the first derivative with respect to time. The strain has a similar equation defining its first derivative with respect to time; however, the magnetic flux density does not and thus must be differentiated within the equation (hence the presence of the $j^{*}$ omega term).

As a final step in establishing the physics of the model, the boundary conditions must be set for each physics domain. Under the "Physics" menu, select "Boundary Settings". In the "Induction Currents" domain, the default boundary condition is magnetic insulation which is fine except when it is expected that the magnetic field lines should pass normal to the surface; in this case, the boundary condition should be changed to electric insulation. In order to model the drive field emanating from the transmit coil, the boundary condition of the surface of the transmit coil should be set to "Surface Current", and the appropriate surface current should be set in the vector description. For example, for a cylinder with the axis along the $\mathrm{z}$ axis, with a surface current of "Js", the appropriate equations to be entered in the vector description of the surface current are 


$$
\begin{aligned}
& J_{x}=-J_{S} * y / \operatorname{sqrt}\left(x^{\wedge} 2+y^{\wedge} 2\right) \\
& \text { and } J_{y}=J_{S} * x / \operatorname{sqrt}\left(x^{\wedge} 2+y^{\wedge} 2\right) .
\end{aligned}
$$

The value of "Js" can be set in the list of model constants (as described in section A.3.2.2). Alternatively, if "Js" varies with frequency, such dependency can be captured by defining the relationship in the "Global Expressions" list (under the "Options" menu, select "Expressions"). In the "Solid, Stress-Strain" domain, symmetry boundary conditions and fixed boundary constraints should be applied as necessary.

\section{A.3.2.4 Mesh Generation}

The next step in developing the model is choosing mesh parameters and meshing the model. Under the "Mesh" menu, select "Free Mesh Parameters". Although many default mesh settings are available, the small dimensions of the thin magnetoelastic subdomain along with the much larger dimensions of the transmit coils make using these default settings difficult. Thus, custom mesh sizes should be defined, with an important parameter being the resolution of narrow regions. For ribbon sensors, a "good" global parameter list is: a max element size scaling factor of 1.5, an element growth rate of 1.6, a mesh curvature factor of 0.7 , a mesh curvature cutoff of 0.04 , and a resolution of narrow regions of 0.1 . This resulted in solutions that were independent of further mesh refinement.

For more complicated magnetoelastic geometries, it is best to not only set global parameters but also to implement parameters that apply to only the magnetoelastic subdomain. For example, the spatially sensitive design described in Chapter 5 was best meshed with the following parameters: element growth rates of 3 (global) and 1.75 
(magnetoelastic subdomain), curvature factor of 0.9 , curvature cutoff of 0.02 , and resolution of narrow regions of 0.36 . This resulted in a total of $\sim 269000$ elements in the model. Note that, for the complex geometries, further refinement of the mesh may result in a slightly different solution; however, a more refined mesh was found to also significantly increase the time required for a solution. Future improvements in computing power or parallel processing may allow for further mesh refinement and solution convergence.

\section{A.3.2.5 Solving and Post-Processing}

Once the model has been meshed it can be solved. There are two ways that the solution can be obtained: one using the parametric solver to sweep through the frequencies, and another using a script that will not only sweep the frequencies but also allow extraction of the desired flux volume integrals so that the induced voltage can be obtained. For the first method, select "Solver Parameters" under the "Solve" menu. Set the solution type to "Frequency Response Analysis" and "Parametric". Change the parameter name to "freq" and input a list of desired frequencies that the solver will sweep through. The solver should be set to the "Direct (Spooles)" linear system solver, although other solvers may also be investigated for computational efficiency. Under the "Advanced" tab, change the solution form to "Weak". Optionally, the "Store Solution on File" box can be checked if it is desired to save the most recent solution when saving the model.

For the second method, select "Solver Manager" under the "Solve" menu. Under the "Script" tab, select "Solve Using Script", and input the following script: 


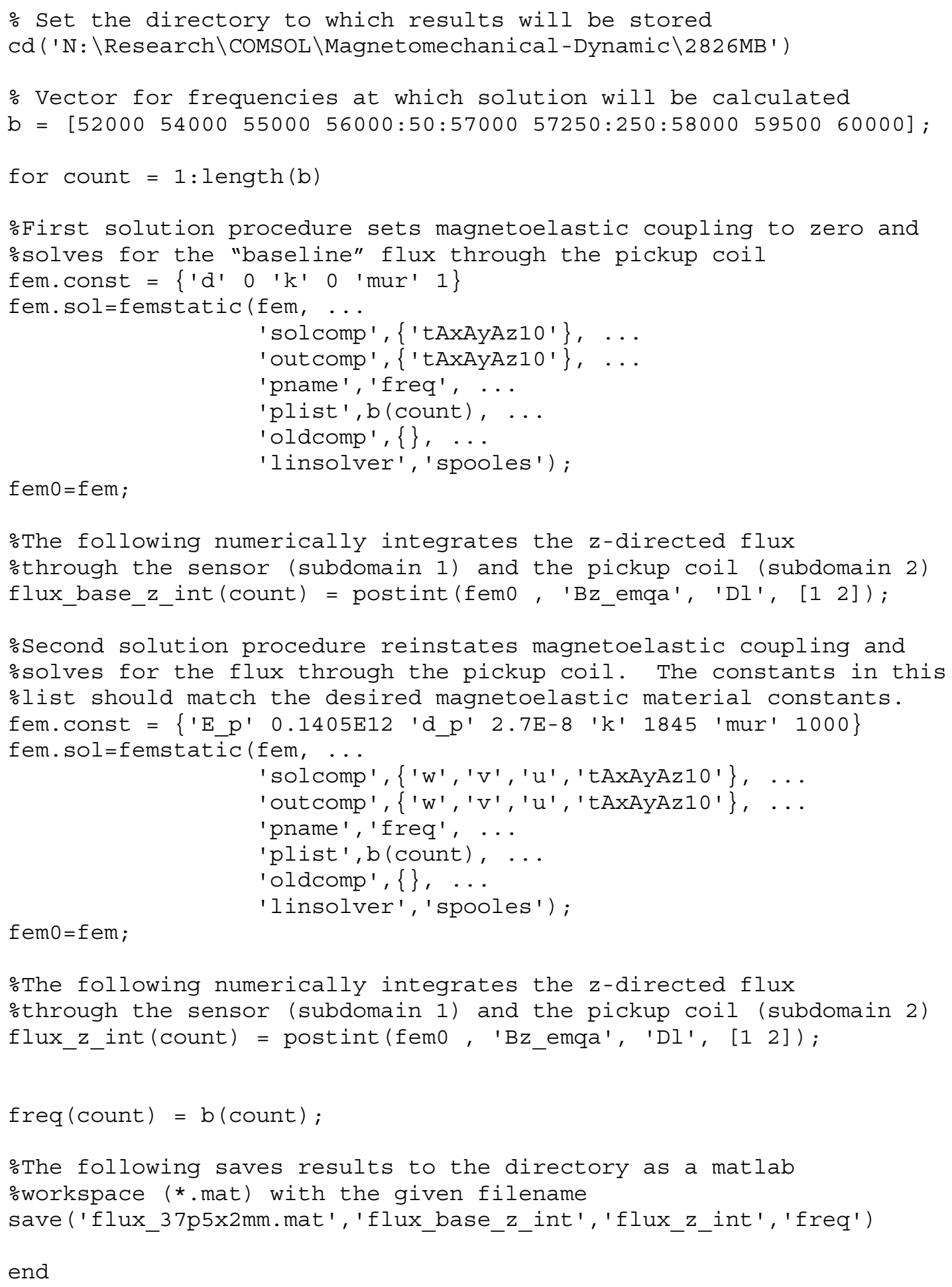

Of course, the script can be modified if other results are desired, especially separately extracting the flux through the sensor and the flux through the coil (which helps to compare FEA and analytical results). 
Once the solver method has been chosen and set up, the model can be solved by pressing the "Solve" button. The results can be post-processed within COMSOL, although only the last frequency solved for can be post-processed if using the second solution method. Post-processed results that are useful include deformed shape, animated mode shapes, and magnetic flux streamlines. Also if using the second solution method, the following MatLab function can be used after the solution workspace has been imported in order to extract the resulting amplitude and phase of the induced voltage:

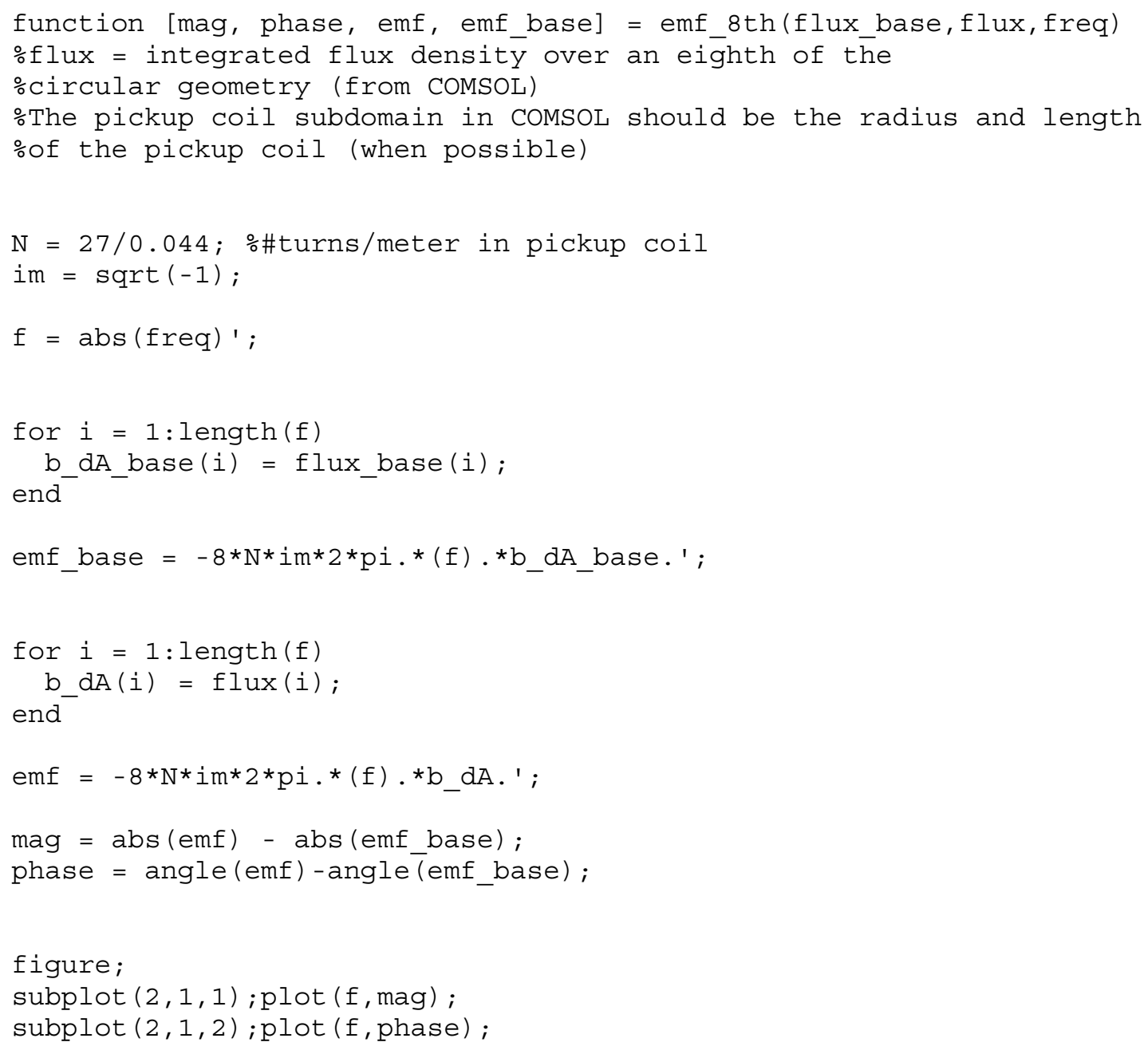

The above script assumes that one-eighth of the overall geometry has been modeled due to symmetry (resulting in the factor of 8 in the electromotive force (EMF) calculations).

Calculations for models with other symmetries should be adjusted appropriately. 


\section{A.4 Comparison of Analytical and FEA Results for Unloaded Ribbon Sensors}

The models presented in sections A.2 and A.3 can be used to analyze simple ribbon sensors that are measured by the coaxial configuration (A) (see section 2.4.2). For the following comparison, the parameters for the as-cast, EDM'd material are used (see Table A-2). Also, the value of "Js" (the surface current applied to the transmit coil in the FEA) is set at $19.5 \mathrm{~A} / \mathrm{m}$, which results in a similar magnetic field amplitude $(H)$ in the analytical model. Key results for the two analysis tools for various geometries and materials are presented in Table A-3.

Table A-3: Selected results for ribbon sensors using the analytical and FEA models

\begin{tabular}{|c|c|c|c|c|c|c|c|c|c|c|}
\hline \multirow{2}{*}{$\begin{array}{c}\text { Alloy } \\
\text { (as-cast) }\end{array}$} & \multirow{2}{*}{$\begin{array}{c}\text { Length } \\
(\mathrm{mm})\end{array}$} & \multirow{2}{*}{$\begin{array}{l}\text { Width } \\
\text { (mm) }\end{array}$} & \multicolumn{2}{|c|}{$\begin{array}{c}\text { Tip Displacement } \\
(\mu \mathrm{m})\end{array}$} & \multicolumn{2}{|c|}{$\begin{array}{c}\text { Resonant Freq. } \\
(\mathrm{kHz})\end{array}$} & \multicolumn{2}{|c|}{$\begin{array}{c}\text { Anti-Resonant } \\
\text { Freq. (kHz) }\end{array}$} & \multicolumn{2}{|c|}{$\begin{array}{c}\text { Signal Amplitude } \\
(\mathrm{mV})\end{array}$} \\
\hline & & & An. & FEA & An. & FEA & An. & FEA & An. & FEA \\
\hline \multirow{10}{*}{ 2826MB } & \multirow{5}{*}{15} & 0.5 & 1.194 & 1.18 & \multirow{5}{*}{140.365} & 141.90 & \multirow{5}{*}{142.447} & 142.20 & 47.5 & 31.1 \\
\hline & & 1 & 1.062 & 1.04 & & 142.42 & & 142.75 & 84.5 & 48.7 \\
\hline & & 2 & 0.926 & 0.91 & & 142.95 & & 143.25 & 147.5 & 73.6 \\
\hline & & 3 & 0.848 & 0.83 & & 143.20 & & 143.50 & 202.7 & 92.4 \\
\hline & & 6 & 0.741 & 0.72 & & 143.20 & & 143.50 & 353.6 & 135.2 \\
\hline & \multirow{5}{*}{37.5} & 0.5 & 1.454 & 1.430 & \multirow{5}{*}{56.063} & 56.25 & \multirow{5}{*}{57.354} & 56.55 & 9.7 & 18.0 \\
\hline & & 1 & 1.414 & 1.398 & & 56.30 & & 56.60 & 18.9 & 34.0 \\
\hline & & 2 & 1.356 & 1.310 & & 56.45 & & 56.75 & 36.3 & 61.1 \\
\hline & & 3 & 1.316 & 1.282 & & 56.50 & & 56.80 & 52.8 & 86.0 \\
\hline & & 6 & 1.247 & 1.182 & & 56.65 & & 56.95 & 100.1 & 148.0 \\
\hline \multirow{10}{*}{$2605 \mathrm{CO}$} & \multirow{5}{*}{15} & 0.5 & 0.341 & 0.343 & \multirow{5}{*}{120.754} & 125.0 & \multirow{5}{*}{124.544} & 127.2 & 14.5 & 14.1 \\
\hline & & 1 & 0.338 & 0.340 & & 127.5 & & 129.6 & 28.8 & 28.2 \\
\hline & & 2 & 0.335 & 0.335 & & 130.6 & & 132.8 & 57.1 & 57.6 \\
\hline & & 3 & 0.332 & 0.332 & & 132.7 & & 134.8 & 84.9 & 87.1 \\
\hline & & 6 & 0.327 & 0.327 & & 136.4 & & 138.4 & 167.1 & 173.2 \\
\hline & \multirow{5}{*}{37.5} & 0.5 & 0.342 & 0.345 & \multirow{5}{*}{47.651} & 48.0 & \multirow{5}{*}{50.865} & 50.2 & 2.7 & 5.5 \\
\hline & & 1 & 0.342 & 0.345 & & 48.2 & & 50.4 & 5.3 & 11.0 \\
\hline & & 2 & 0.341 & 0.344 & & 48.6 & & 50.8 & 10.6 & 22.1 \\
\hline & & 3 & 0.340 & 0.344 & & 48.8 & & 51.0 & 15.8 & 33.3 \\
\hline & & 6 & 0.340 & 0.344 & & 49.4 & & 51.6 & 31.6 & 67.1 \\
\hline \multirow{10}{*}{$2605 \mathrm{SA} 1$} & \multirow{5}{*}{15} & 0.5 & 0.417 & 0.399 & \multirow{5}{*}{125.807} & 131.4 & \multirow{5}{*}{129.182} & 133.0 & 32.3 & 15.7 \\
\hline & & 1 & 0.343 & 0.320 & & 132.8 & & 134.6 & 53.0 & 20.9 \\
\hline & & 2 & 0.279 & 0.255 & & 134.2 & & 135.8 & 86.1 & 26.7 \\
\hline & & 3 & 0.246 & 0.224 & & 134.8 & & 136.4 & 114.2 & 30.8 \\
\hline & & 6 & 0.204 & 0.182 & & 135.0 & & 136.8 & 189.6 & 40.0 \\
\hline & \multirow{5}{*}{37.5} & 0.5 & 0.605 & 0.592 & \multirow{5}{*}{49.798} & 50.4 & \multirow{5}{*}{52.460} & 52.2 & 7.5 & 12.9 \\
\hline & & 1 & 0.569 & 0.550 & & 50.8 & & 52.5 & 14.1 & 22.4 \\
\hline & & 2 & 0.524 & 0.491 & & 51.3 & & 53.0 & 25.9 & 36.7 \\
\hline & & 3 & 0.495 & 0.460 & & 51.6 & & 53.2 & 36.7 & 48.1 \\
\hline & & 6 & 0.447 & 0.398 & & 52.0 & & 53.8 & 66.3 & 73.1 \\
\hline
\end{tabular}




\section{A.5 Potential Improvements}

A number of potential improvements could be investigated for the models presented in this appendix. For the analytical model, it may be possible to improve the utility of the model by developing an analytical treatment for determining the shape function for a given sensor permeability and dimensions, rather than determining the parameters A and $\mathrm{B}$ in equation (A-51) using a magnetostatic finite element model. Agreement with the magnetomechanical FEA model may be improved by more accurate calculation of the value of $S$ for different sensor geometries, and by inclusion of transverse strain and magnetic effects (e.g. with Poisson's ratio for transverse strain in the presence of longitudinal strain).

For the FEA model, modeling the sensor material as an anisotropic material may result in better predictive accuracy, especially in complex sensor geometries. Additionally, future improvements in computing powers may allow a more detailed mesh refinement study for the complex sensor geometries. If it is determined that the model is not capturing important physical effects, the model may be augmented to include higher order effects, including eddy current losses, frequency response of the transmit and receive coils, magnetic hysteresis effects, and nonlinear effects due to variation in material properties with different applied magnetic fields. Finally, another avenue for

improvement of the predictive accuracy of both models is the further study and cataloguing of material properties after different fabrication processes and with different feature sizes. 


\section{Appendix B}

\section{Wireless Magnetoelastic Ultrasound Generation}

This appendix presents a closer look at a preliminary investigation into the wireless magnetoelastic generation of ultrasound. Previous work with magnetoelastic sensors has discussed utilization of a microphone or hydrophone pickup for wireless communication (as opposed to the inductive pickup discussed elsewhere in this work) [Jai00, Jai01_2]. However, the applications remained in the wireless magnetoelastic sensing arena, and no work was done for the express purpose of generating ultrasonic energy.

Ultrasound is frequently applied for medical diagnostics and therapy. However, there have been no reports of wirelessly-generated ultrasound. All ultrasound systems discussed in the literature are either catheter-based (for example, intravenous ultrasound (IVUS) systems [Abi98]) or deliver the ultrasound from the surface of the body down to the required depth. However, ultrasound that is generated wirelessly at the site of the implant could offer potential benefits. First, a wireless ultrasound generator that is integrated with an implant would no longer require catheterization for the local application of ultrasound. Secondly, the implanted ultrasound generator can potentially deliver targeted or focused acoustic energy much more easily than an externally applied ultrasound generator. This can minimize invasiveness while still potentially reaping the benefits of catheter-based ultrasound systems (namely, the ability to deliver highfrequency ultrasound directly to the desired site).

Potential applications for wireless ultrasound generation are often therapeutic in nature. Thrombolysis ("clot busting") is one such application. A large amount of 
research has been done in catheter-based or externally applied ultrasound that has shown the ability to dissolve clots [Sie08]. Medical devices that offer compelling needs for onboard, wireless ultrasonic thrombolysis include cardiac stents (especially because drug eluting stents have shown an increased potential for subacute and late thrombosis [McF04, Vir04, Pfi06, Fer06, Che07]) and vena cava filters (which "snare" deep vein clots before they pass to the lungs, and could be cleared rapidly with onboard wireless ultrasound). Having clot busting capability in the device itself may negate (or relax) the need for systemic anticoagulant and antiplatelet therapy and the associated bleeding risks.

Another application that has received a lot of attention is the use of ultrasound in cancer therapy. Two main methods have been investigated - tumor ablation with high intensity focused ultrasound (HIFU) and targeted drug delivery [Wu03, Tac01]. Microbubble destruction and permeation of target cells are the two most often proposed methods for targeted drug delivery. To this point, the ultrasound has been applied externally with appropriate frequency selection and focusing design, or it has been applied with and intercavity probe. In general, cancers are not treated with implants, so on-board wireless ultrasound generation for cancer treatment is not generally beneficial. However, often specific implanted devices (such as biliary, ureteral, and esophageal stents) are used in the palliation of symptoms (such as constricted ducts) that are associated with tumor growth. In these cases, embedded ultrasound generation - either for tumor ablation or targeted drug delivery - may provide an opportunity to prevent excessive cancer ingrowth. On-board wireless ultrasound generation may also be used for targeted delivery of smooth muscle cell inhibitors (like rapamycin) to prevent restenosis in cardiac stents. 
Design possibilities for generating ultrasound using magnetoelastic transduction with amorphous alloys are explored in the following section. The preliminary results from a prototype design are then presented.

\section{B.1 Design Concepts}

\section{B.1.1 Piezomagnetic Disc Stack}

A typical design for an ultrasound transducer is a single (or stack) of piezoelectric discs. This architecture could be mimicked with magnetoelastic material replacing the piezoelectric material. Amorphous alloys are typically available in thin foils $(\sim 25 \mathrm{~mm})$; stacks of discs fabricated from these foils could be fabricated as a way to offer more flexibility in the resonant frequency, bandwidth, and power efficiency of the design.

For instance, the layout shown in Fig. B-1 can be fabricated using photochemical machining or an appropriate alternative. Subsequent to patterning, the material can be thermally treated as necessary (see sections 2.2.1.1 and 5.2) and then coated with Parylene. Then, the square handling frame bordering each disc can be used to align the

discs in a corner fixture. The discs can then be Parylene-Parylene bonded to form a stack.

The handling frame can also be used to align the stack to similar features on the medical device. The stack can then be parylene-parylene bonded to the medical device. Finally, the handling frame can be trimmed away, leaving only the circular center section as the ultrasonic transducer. 
In order to estimate the acoustic pressures that can be generated by such a stack, the magnetomechanical FEA tool described in section 2.2.3 can be further augmented with an acoustic physics domain, a procedure which is well documented in the COMSOL user guide. An axisymmetric acousto-magneto-mechanical model of $2826 \mathrm{MB}$ discs $(1 \mathrm{~mm}$ diameter) each separated by $1 \mu \mathrm{m}$ of Parylene is implemented in COMSOL. The model estimates a pressure of $35 \mathrm{~Pa}$ generated at $1.24 \mathrm{MHz}$ for a 15-layer stack (in water), while an estimated pressure of $75 \mathrm{~Pa}$ is generated at $0.64 \mathrm{MHz}$ for a 30-layer stack (in water, Fig. B-2).

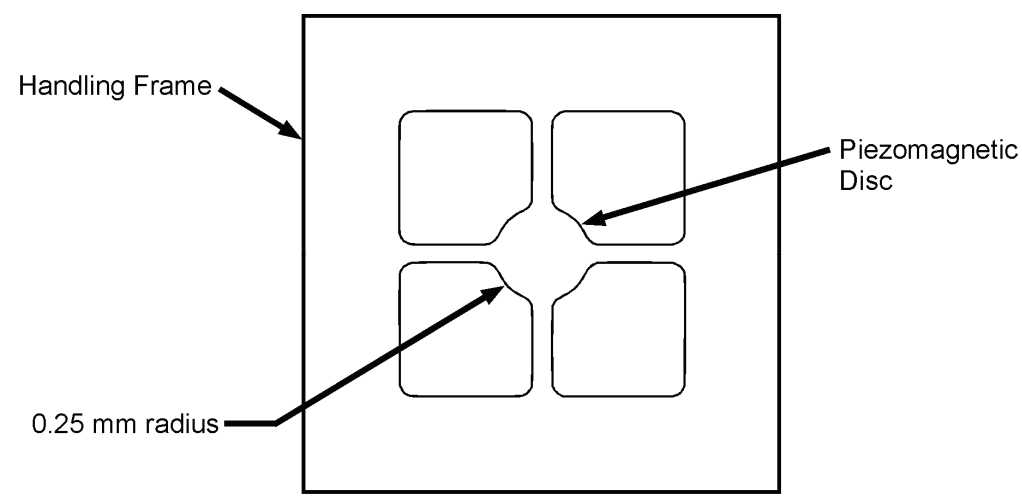

Fig. B-1: Piezomagnetic disc layout.

$640 \mathrm{kHz}$

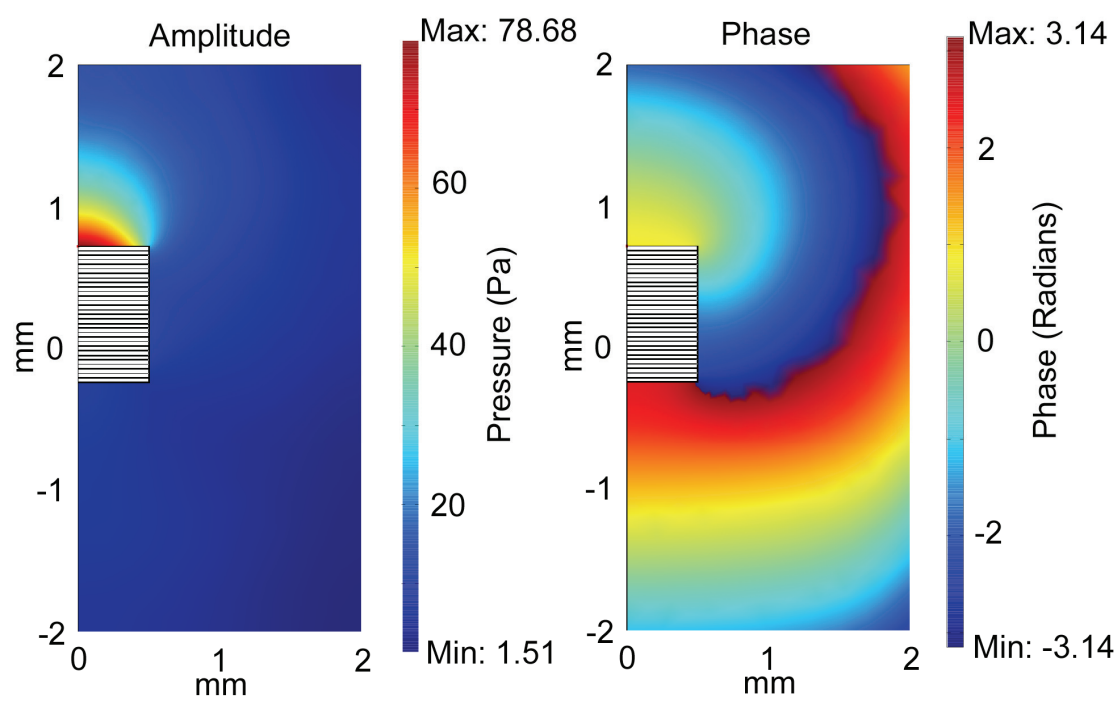

Fig. B-2: LEFT: Pressure amplitude of 30-layer stack, operating at resonance in water. RIGHT: Pressure phase of 30-layer stack. 


\section{B.1.2 "Bent-Beam" Actuator}

An alternative design concept utilizes a method for generating out of plane motion from an initially planar structure. A layout is proposed in Fig. B-3. By bending up the active regions of the device, bending modes can be excited that result in pressure generated at the active area. The active regions can be bent out-of-plane by using the thermal treatments suggested in Chapters 4 and 5 (where such treatments were used to add curvature to the sensors). A 3-D acousto-magnetomechanical model of the layout is implemented in COMSOL using 2826MB material properties. Figure B-4 shows the bending deformation estimated by the model, which predicts a pressure of $21 \mathrm{kPa}$ is generated at $99.1 \mathrm{kHz}$ for this particular design if operated in water.

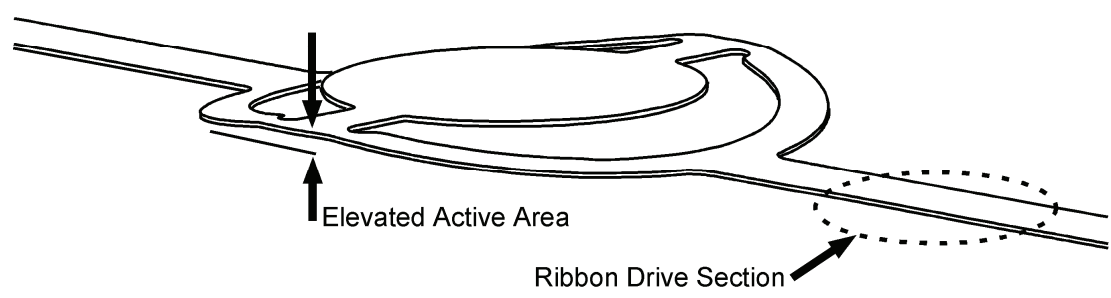

Fig. B-3: Bent-Beam Actuator conceptual schematic. Planar ribbon drive sections actuate the elevated active area, resulting in out-of-plane motion.
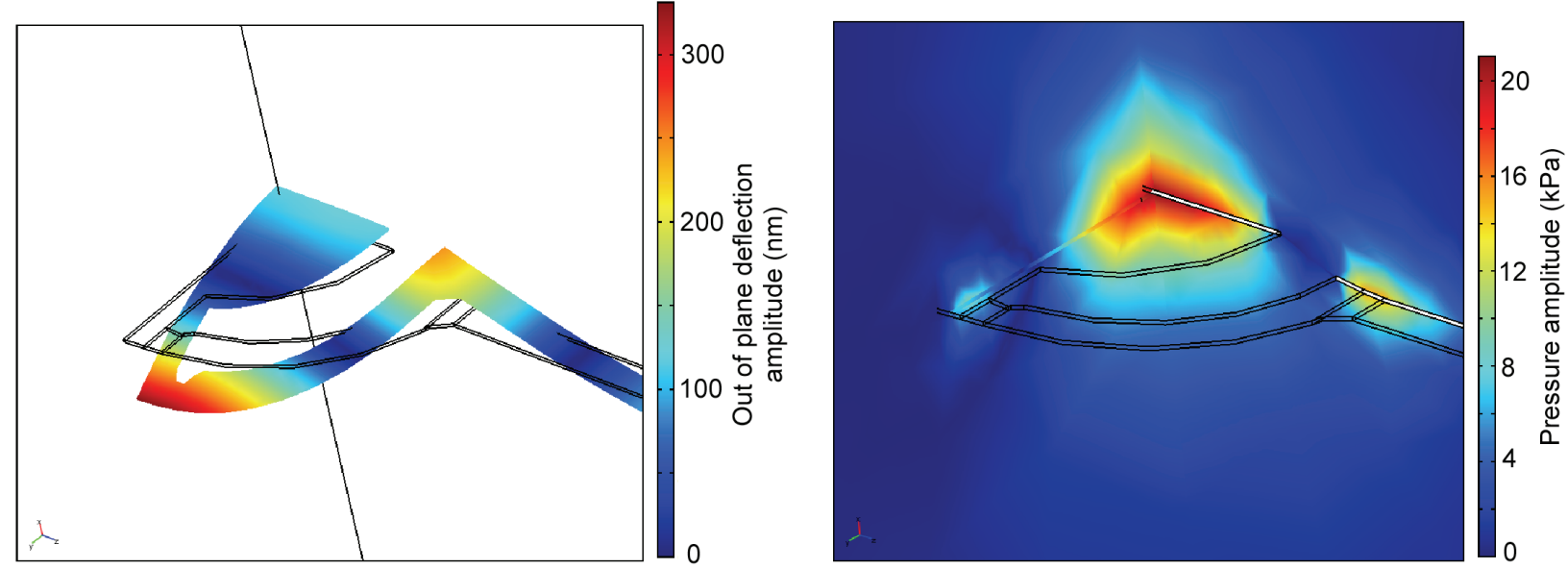

Fig. B-4: LEFT: Deformation of ultrasound device, with out of plane deflection (deformation not to scale). RIGHT: Corresponding pressure amplitude. 


\section{B.2 Wireless Ultrasound Prototype Device}

\section{B.2.1 Experimental}

A simple prototype design utilizing the "bent-beam" actuator concept was fabricated from Metglas ${ }^{\mathrm{TM}}$ 2605SA1, using micro-electro-discharge machining. The layout is shown in Fig. B-5. Transverse field annealing (30 minutes at $413^{\circ} \mathrm{C}$ in a $1.5 \mathrm{kOe}$ transverse field) was used to improve resonant magnetoelastic signal by a factor of 3 (this should also improve the ultrasound generation capability by improving effective magnetostrictivity). Thermal annealing, along with $500 \mu \mathrm{m}$ thick pieces of metal and clamps, was used to elevate the end sections out-of-plane, as schematically illustrated in

Fig. B-6. The end pads were then affixed (with cyanoacrylate) to an Elgiloy ${ }^{\mathrm{TM}}$ strip (100 $\mu \mathrm{m}$ thick).

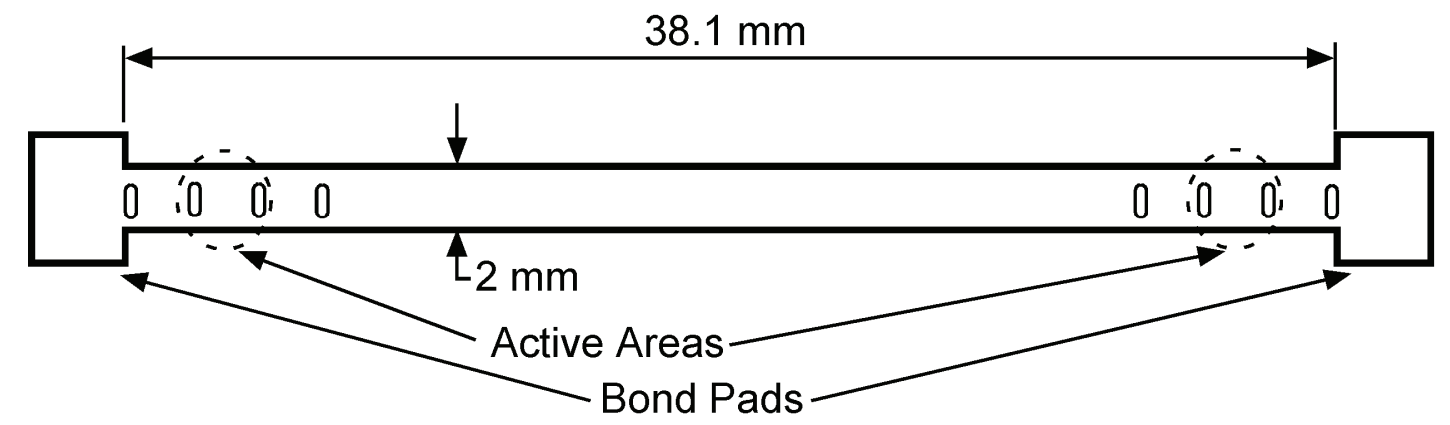

Fig. B-5: Prototype layout. The slots define the active areas, and bond pads for affixing the device to a substrate are at the ends.

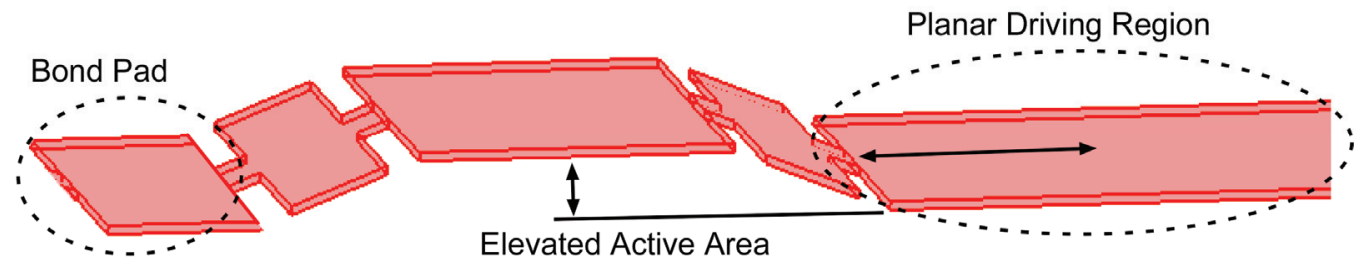

Fig. B-6: Active areas are elevated out of plane using thermal annealing (1 hour at $375^{\circ} \mathrm{C}$ while a fixture provides the desired final shape). 
A Larson-Davis Model 2520 (S/N 1372) 1/4" microphone was then placed above the elevated end sections of the actuator to evaluate acoustic signals originating from the ends. The actuator was first tested in air, with the microphone placed at different distances above the end. Then, the actuator was placed in a $50 \mathrm{~mL}$ Becton-Dickinson (BD) polycarbonate sample vial filled with deionized water. The microphone was placed against the outer surface of the sample holder, aligned with the end of the actuator. Representative frequency response traces are in Fig. B-7. A significant acoustic pressure was measured above the active areas at the resonant frequency of the device. The pressure decreased slightly as the distance between the microphone and active area increased. The measured pressure was significantly decreased by the presence of the water and sample vial, likely due to acoustic scattering and reflection caused by the surface interfaces between the active area and microphone. Future tests may provide better results with the use of a hydrophone in such instances.

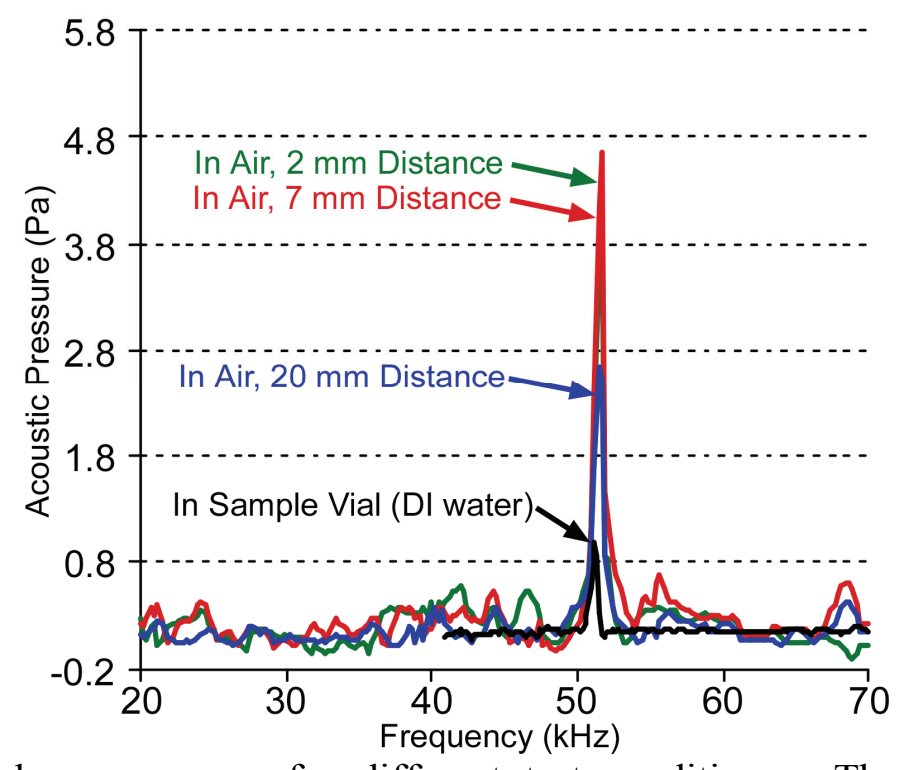

Fig. B-7: Microphone response for different test conditions. The microphone was suspended over the active area of the device and a network analyzer measured the response versus frequency as the drive signal was simultaneously swept. 


\section{B.2.2 Analysis}

Using COMSOL, the actuator was modeled in coupled magnetic, mechanical, and acoustic domains. The subdomain around the actuator was modeled with the acoustic properties of water. In this way, the acoustic pressure generated by the actuator in water could be estimated. Note that $2826 \mathrm{MB}$ material properties were used for the actuator, while the prototype device was fabricated from 2605SA1. In the tested configuration, the model estimates that $14.7 \mathrm{kPa}$ of sound pressure can be generated at $48.3 \mathrm{kHz}$ at the surface of the active area (Fig. B-8).
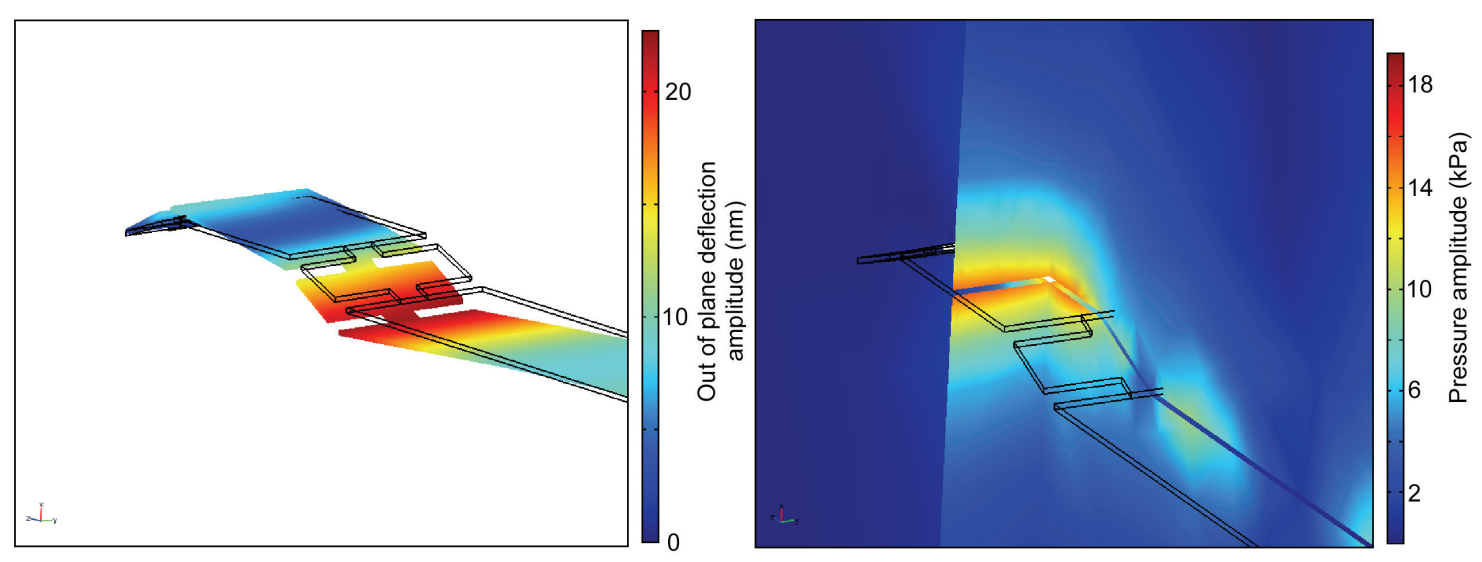

Fig. B-8: LEFT: Estimated deformation amplitude of prototype at resonance. RIGHT: Corresponding acoustic pressure amplitude.

\section{B.3 Discussion}

Use of magnetoelastic resonators as actuators for the wireless generation of mechanical motion and ultrasound is a mostly unexplored topic at the meso- and microscales. Ultrasound can be put to use in a number of ways and in a number of areas, including medical diagnostics and therapy as well as structural health monitoring. 
Development of a method for wirelessly generating ultrasound energy may provide benefits in targeted, focused therapy or in the ability to apply ultrasound in difficult-toaccess locations. The concepts and preliminary experimental results described in this appendix illustrate the feasibility of using magnetoelastic resonators as wireless ultrasound generators. Continued future work should include development of a rigorous experimental setup, as well as specification of performance and design for key applications. 


\section{REFERENCES}

-235 - 
[Abi98] A. Abizaid, G. Mintz, A. Pichard, K. Kent, L. Satler, C. Walsh, J. Popma, M. Leon, "Clinical, Intravascular Ultrasound, and Quantitative Angiographic Determinants of the Coronary Flow Reserve Before and After Percutaneous Transluminal Coronary Angioplasty," American Journal of Cardiology, Vol. 82, No. 4, pp. 423-428, 1998.

[And82] P. Anderson III, "Magnetomechanical Coupling, $\Delta$ E Effect, and Permeability in FeSiB and FeNiMoB Alloys," J. Appl. Phys., Vol. 53, No. 11, pp. 8101$8103,1982$.

[Ang02] J. Angeles, S. Ostrovskaya, "The Proportional-Damping Matrix of Arbitrarily Damped Linear Mechanical Systems," J. Appl. Mechanics, Vol. 69, pp. 649656, 2002.

[ASM89] ASM Handbook, Vol. 16, ASM International, 1989.

[Bar97] G. Barbalias, D. Siablis, E. Liatsikos, D. Karnabatidis, S. Yarmentis, K. Bouropoulos, J. Dimopoulos, "Metal Stents: A New Treatment of Malignant Ureteral Obstruction," J. Urology, Vol. 158, pp. 54-8, 1997.

[Bas03] A. Bashar, T. Kazui, N. Washiyama, H. Terada, K. Yamashita, M. Haque, "Mechanical Properties of Various Z-Stent Designs: An Endovascular StentGrafting Perspective," Artificial Organs, Vol. 27, No. 8, pp. 714-21, 2003.

[Ben05] J. Benatar, FEM Implementation of Magnetostrictive-Based Applications, MS thesis, University of Maryland, 2005.

[Bin00] M. Binhack, W. Buff, S. Klett, M. Hamsch, "A Combination of SAWResonators and Conventional Sensing Elements for Wireless Passive Remote Sensing," IEEE Ultrasonics Symposium, pp. 495-498, 2000.

[Bon98] L. Bondemark, J. Kurol, A. Larsson, "Long-term effects of orthodontic magnets on human buccal mucosa - a clinical, histological and immunohistochemical study," European J. of Orthod., Vol. 20, pp. 211-218, 1998.

[Bul01] W.-E. Bulst, G. Fischerauer, L. Reindl, "State of the Art in Wireless Sensing with Surface Acoustic Waves," IEEE Trans. on Ind. Elec., Vol. 48, No.2, pp. 265-71, 2001.

[Bul03] M. Bultitude, R. Tiptaft, J. Glass, P. Dasgupta, "Management of Encrusted Ureteral Stents Impacted in Upper Tract," J. Urology, Vol. 62, No. 4, pp. 622$6,2003$.

[Cen06] A. Cense, E. Peeters, B. Gottenbos, F. Baaijens, A. Nuijs, M. van Dongen, "Mechanical Properties and Failure of Streptococcus mutans Biofilms, 
Studied Using a Microindentation Device," J. Microbiological Methods, Vol. 67, pp. 463-472, 2006.

[Cha94] M. Chaudhry, S. Zubair, "Temperature and heat flux solutions due to steady and non-steady periodic-type surface temperatures in a semi-infinite solid," Heat and Mass Transfer, Vol. 29, pp. 205-10, 1994.

[Cha99] A. Chan, F. Shin, Y. Lam, E. Ng, J. Sung, J. Lau, S. Chung, "A Comparison Study on Physical Properties of Self-Expandable Esophageal Metal Stents," Gastrointestinal Endoscopy, Vol. 49, No. 4, pp. 462-5, 1999.

[Che07] J. Chen, "Very late drug-eluting stent thrombosis and cardiogenic shock: 1,249 days after implantation," J. Invasive Cardiol., Vol 19, pp. E381-E384, 2007.

[Cla89] A. Clark, M. Wun-Fogle, "A New Method of Magnetostrictivity and Magnetostriction Measurement," IEEE Trans. on Magnetics, Vol. 25, No. 5, pp. 3611-3, 1989.

[Cla02] M. Classen, G. Tytgat, C. Lightdale, Gastroenterological Endoscopy, Thieme, 2002.

[Coe90] P. Coene, A. Groen, J. Cheng, M. Out, G. Tytgat, K. Huibregtse, "Clogging of Biliary Endoprostheses: A New Perspective,” Gut, Vol. 31, pp. 913-917, 1990.

[Col07] L. Colombato, "The Role of Transjugular Intrahepatic Portosystemic Shunt (TIPS) in the Management of Portal Hypertension," J. Clin. Gastroenterol. Vol. 41, Suppl. 3, pp. S344-51, 2007.

[Con05] J. Conway, M. Russo, R. Shrestha, "Endoscopic Stent Insertion into the Gallbladder for Symptomatic Gallbladder Disease in Patients with End-Stage Liver Disease," Gastrointestinal Endoscopy, Vol. 61, No. 1, pp. 32-6, 2005.

[Dar01] C. Darvennes, S. Pardue, "Boundary Effect of a Viscous Fluid on a Longitudinally Vibrating Bar: Theory and Application," J. Acoust. Soc. Am., Vol. 110, No. 1, pp. 216-224, 2001.

[Dav92] P. Davids, A. Groen, E. Rauws, G. Tytgat, K. Huibregtse, "Randomised Trial of Self-Expanding Metal Stents Versus Polyethylene Stents for Distal Malignant Biliary Obstruction," The Lancet, Vol. 340: Dec. 19/26, pp. 148892, 1992.

[DeH04] A. DeHennis, "Remotely-Powered Wireless Monitoring Systems," Doctoral Dissertation, University of Michigan, 2004. 
[DeH06] A. DeHennis, K. Wise, "A Fully Integrated Multisite Pressure Sensor for Wireless Arterial Flow Characterization," J. Microelectromech. Sys., Vol. 15, No. 3, pp. 678-685, 2006.

[DeL00] V. De Ledinghen, B. Person, J. Legoux, A. Le Sidaner, B. Desaint, M. Greff, C. Moesch, G. Grollier, P. Ingrand, D. Sautereau, M. Beauchant, "Prevention of Biliary Stent Occlusion by Ursodexycholic Acid Plus Norfloxacin: A Multicenter Randomized Trial," Dig. Dis. and Sci., Vol. 45, No. 1, pp. 145-50, 2000 .

[Dod95] G. Dodd III, A. Zajko, P. Orons, M. Martin, L. Eichner, L. Santaguida, "Detection of Transjugular Intrahepatic Portosystemic Shunt Dysfunction: Value of Duplex Doppler Sonography," American J. of Roentgenology, Vol. 164, pp. 1119-24, 1995.

[Don07] G. Donelli, E.Guaglianone, R. Di Rosa, F. Fiocca, A. Basoli, "Plastic Biliary Stent Occlusion: Factors Involved and Possible Preventive Approaches," Clinical Med. Res., Vol. 5, No. 1, pp. 53-60, 2007.

[Dow91] N. Dowidar, H. Kolmos, H. Lyon, P. Matzen, "Clogging of Biliary Endoprostheses: A Morphologic and Bacteriologic," Scandinavian Journal of Gastroenterology, Vol. 26, No. 11, pp. 1137-44, 1991.

[Dow92] N. Dowidar, H. Kolmos, P. Matzen, "Experimental Clogging of Biliary Endoprostheses. Role of Bacteria, Endoprosthesis Material, and Design," Scandinavian Journal of Gastroenterology, Vol. 27, No. 1, pp. 77-80, 1992.

[Dur92] C. Durney, "Interactions Between Electromagnetic Fields and Biological Systems," Annals of the New York Academy of Sciences, Vol. 649, pp. 19-34, 1992.

[Elg08] Elgiloy ${ }^{\mathrm{TM}}$ Specialty Metals. Available online at http://www.elgiloy.com

[Eng00] G. Engdahl (ed.), Handbook of Giant Magnetostrictive Materials, Academic Press, 2000.

[Fax01] D. Faxon (ed.), Restenosis: A Guide to Therapy, Martin Dunitz Ltd., 2001.

[FDA98] Available online at www.fda.gov/cdrh/ode/bistent.pdf. Accessed on March 20, 2008.

[Fer96] V. Ferrari, D. Marioli, A. Taroni, E. Ranucci, P. Ferruti, "Development and Applications of Mass Sensors Based on Flexural Resonances in Alumina Beams," IEEE Trans. on Ultrasonics, Ferroelectrics, and Freq. Cont., Vol. 43, No. 4, pp. 601-8, 1996. 
[Fer06] F. Feres, J. Costa Jr., A. Abizaid, "Very late thrombosis after drug-eluting stents," Catheter Cardiovasc. Interv., Vol. 68, pp. 83-88, 2006.

[Fle77] D.G. Fleming, W.K. Ko, M.R. Neuman, Indwelling and Implantable Pressure Transducers, CRC Press, 1977.

[Fon06] M. Fonseca, M. Allen, J. Kroh, J. White, "Flexible Wireless Passive Pressure Sensors for Biomedical Applications," Hilton Head 2006: Sol. State Sens. Act. Microsys. Workshop, pp. 37-42, June 2006.

[Fra96] F. Frattaroli, D. Reggio, A. Guadalaxara, "Benign Biliary Strictures: A Review of 21 Years of Experience," J. Am. Coll. Surg., Vol. 183, No. 5, pp. 506-13, 1996.

[Gin03] G. Ginsberg, C. Cope, J. Shah, T. Martin, A. Carty, P. Habecker, C. Kaufmann, C. Clerc, J. Nuutinen, P. Tormala, "In Vivo Evaluation of a New Bioabsorbable Self-Expanding Biliary Stent," Gastrointestinal Endoscopy, Vol. 58, No. 5, pp. 777-84, 2003.

[Gou04] X. Gou, Y. Yang, X. Zheng, "Analytic Expression of Magnetic Field Distribution of Rectangular Permanent Magnets," Applied Mathematics and Mechanics, Vol. 25, No. 3, pp. 297-306, 2004.

[Gre04] L. Grenacher, E. Ganger, A. Lubienski, M. Dux, G. Kauffman, G. Richter, "Experimental Functional Analysis of Self-Expanding Stents Using a New Developed Ex Vivo Model," Investigative Radiology, Vol. 39, No. 6, pp. 37483, 2004.

[Gre07] S. Green, M. Richardson, F. Shariff, Y.B. Gianchandani, "Photochemically Patterned Biliary Stents with Integrated Permanent Magnets and Deformable Assembly Features for Wireless Magnetoelastic Tissue Growth Sensing," Solid-State Sensors, Actuators and Microsystems Conference, Transducers 2007, pp. 213-217, June 2007.

[Gre08] S. Green, Y.B. Gianchandani, "Wireless Biliary Stent System with WishboneArray Resonant Magnetoelastic (WARM) Sensor and Conformal Magnetic Layer," Hilton Head 2008: Sol. State Sens. Act. Microsys. Workshop, pp. 158-161, 2008.

[Gre09] S. Green, Y.B. Gianchandani, "Wireless Magnetoelastic Monitoring of Biliary Stents," J. of Microelectromechanical Systems, Vol. 18, No. 1, pp. 64-78.

[Gri98] C. Grimes, P. Stoyanov, "A Remotely Interrogatable Magnetochemical Sensor for Environmental Monitoring,” Proc. IEEE Aerospace Conf., pp. 87-92, 1998. 
[Gri00] C. Grimes, D. Kouzoudis, C. Mungle, "Simultaneous Measurement of Liquid Density and Viscosity Using Remote Query Magnetoelastic Sensors," Rev. of Scientific Instruments, Vol. 71, No. 10, pp. 3822-3824, 2000.

[Gri02] C. Grimes, C. Mungle, K. Zeng, M. Jain, W. Dreschel, M. Paulose, K. Ong, "Wireless Magnetoelastic Resonance Sensors: A Critical Review," Sensors, Vol. 2, pp. 294-313, 2002.

[Han05] J. Hanus, J. Zahora, "Measurement and Comparison of Mechanical Properties of Nitinol Stents," Physica Scripta, Vol. T118, pp. 264-7, 2005.

[Hem98] K. Hemmerich, "Accelerated Aging: General Aging Theory and Simplified Protocol for Accelerated Aging of Medical Devices," Medical Plastics and Biomaterials Magazine, July 1998.

[Hen06] K. Hendlin, K. Dockendorf, C. Horn, N. Pshon, B. Lund, M. Monga, "Ureteral Stents: Coil Strength and Durometer," J. Urology, Vol. 68, No. 1, pp. 42-5, 2006.

[Her88] A. Hernando, M. Vazquez, J. Barandiaran, "Metallic Glasses and Sensing Applications,” J. Phys. E: Sci. Instrum., Vol. 21, pp. 1129-1139, 1988.

[Her03] G. Herzer, "Magnetic Materials for Electronic Article Surveillance," J. of Magnetism and Magnetic Materials, Vols. 254-255, pp. 598-602, 2003.

[Hof89] R. Hofmann, R. Hartung, "Ureteral Stents - Materials and New Forms," World J. Urology, Vol. 7, pp. 154-7, 1989.

[How91] P. Howard, G. Murphy, R. Dowling, "Gall Bladder Emptying Patterns in Response to a Normal Meal in Healthy Subjects and Patients with Gall Stones: Ultrasound Study," Gut, Vol. 32, pp. 1406-11, 1991.

[IEE71] IEEE Standard on Magnetostrictive Materials: Piezomagnetic Nomenclature. IEEE Std. 319-1971, 1971.

[Jai00] M. Jain, S. Schmidt, K. Ong, C. Mungle, C. Grimes, "Magnetoacoustic Remote Query Temperature and Humidity Sensors," Smart Mater. Struct., Vol. 9, pp. 502-10, 2000.

[Jai01] M. Jain, Q. Cai, C. Grimes, "A Wireless Micro-Sensor for Simultaneous Measurement of pH, Temperature, and Pressure," Smart Mat. and Struc., Vol. 10, pp. 347-353, 2001.

[Jai01_2] M. Jain, S. Schmidt, C. Grimes, "Magneto-acoustic Sensors for Measurement of Liquid Temperature, Viscosity and Density," Applied Acoustics, Vol. 62, pp. 1001-11, 2001. 
[Joh99] D. Johnston, "Special Considerations in Interpreting Liver Function Tests," American Family Physician, Vol. 59, No. 8, pp. 2223-32, 1999.

[Jun01] D. Jungst, A. Niemeyer, I. Muller, B. Zundt, G. Meyer, M. Wilhelmi, R. del Pozo, "Mucin and Phospholipids Determine the Viscosity of Gallbladder Bile in Patients with Gallstones," World J. of Gastroenterology, Vol. 7, No. 2, pp. 203-207, 2001.

[Kan97] R. Kanterman, M. Darcy, W. Middleton, K. Sterling, S. Teefey, T. Pilgram, "Doppler Sonography Findings Associated with Transjugular Intrahepatic Portosystemic Shunt Malfunction," American J. of Roentgenology, Vol. 168, pp. 467-72, 1997.

[Kim97] J. Kim, K. Wise, J. Grizzle, "In-Situ Polymer Buildup Monitoring in Plasma Etching Systems," Proc. IEEE Int. Symp. on Semicon. Manuf., pp. B37-B40, Oct. 1997.

[Kim99] M. Hyoung Kim, K. Shik Lee, S. Ho Lim, "Magnetostriction Measurements of Metallic Glass Ribbon by Fiber-Optic Mach-Zehnder Interferometry," $J$. Mag. and Mag. Mat., Vol. 191, pp. 107-112, 1999.

[Kim05] S.-J. Kim, T. Ono, M. Esashi, "Capacitive Resonant Mass Sensor with LC Resonant Circuit for Use in Atmosphere," Solid-State Sensors, Actuators and Microsystems Conference, Transducers 2005, pp. 256-60, June 2005.

[KimH05] H. Kim, K. Najafi, "Characterization of Low-Temperature Wafer Bonding Using Thin-Film Parylene," J. Microelectromechanical Sys., Vol. 14, No. 6, pp. 1347-1355, 2005.

[Ko03] C. Ko, "Biliary Sludge and Cholecystitis," Best Practice \& Research Clinical Gastroenterology, Vol. 17, No. 3, pp. 383-96, 2003.

[Koz00] R. Kozarek, "Metallic Biliary Stents for Malignant Obstructive Jaundice: A Review," World J. Gastroenterology, Vol. 6, No. 5, pp. 643-6, 2000.

[Kun04] T. Kundu (ed.), Ultrasonic Nondestructive Evaluation: Engineering and Biological Material Characterization, CRC Press, Boca Raton, FL, 2004.

[Lag97] L. Lagorce, M. Allen, "Magnetic and Mechanical Properties of Micromachined Strontium Ferrite/Polyimide Composites," J. Microelectromech. Sys., Vol. 6, No. 4, pp. 307-12, 1997.

[Lam86] J. Lammer, G. Stoffler, W. Petek, H. Hofler, "In Vitro Long-Term Perfusion of Different Materials for Biliary Endoprostheses," Investigative Radiology, Vol. 21, pp. 329-31, 1986. 
[Lea05] M. Leach, Introduction to Electroacoustics and Audio Amplifier Design, Kendall Hunt, 2005.

[Lia07] E. Liatsikos, G. Kagadis, D. Karnabatidis, K. Katsanos, Z. Papathanassiou, C. Constantinides, P. Perimenis, G. Nikiforidis, J. Stolzenburg, D. Siablis, "Application of Self-Expandable Metal Stents for Ureteroileal Anastomatic Strictures: Long-Term Results,” J. Urology, Vol. 178, pp. 169-73, 2007.

[Lib96] E. Libby, J. Leung, "Prevention of Biliary Stent Clogging: A Clinical Review," Am. J. of Gastroenterology, Vol. 91, No. 7, pp. 1301-7, 1996.

[Lid04] D. Lide (ed.), CRC Handbook of Chemistry and Physics, $85^{\text {th }}$ ed., CRC Press, 2004.

[Lin95] J.-J. Lin, T.-P. Perng, "Embrittlement of Amorphous $\mathrm{Fe}_{40} \mathrm{Ni}_{38} \mathrm{Mo}_{4} \mathrm{~B}_{18}$ Alloy by Electrolytic Hydrogen," Met. and Mat. Trans. A, Vol. 26, No. 1, pp. 197$201,1995$.

[Liv82] J. D. Livingston, "Magnetomechanical Properties of Amorphous Metals," Phys. Stat. Sol. (A), Vol. 70, pp. 591-596, 1982.

[Lo95] C.-M. Lo, C. Keese, I. Giaever, "Impedance Analysis of MDCK Cells Measured by Electric Cell-Substrate Impedance Sensing," Biophysical Journal, Vol. 69, pp. 2800-7, 1995.

[Lyn07] M. Lynch, K. Ghani, I. Frost, K. Anson, "Preventing the Forgotten Ureteral Stent: Implementation of a Web-Based Stent Registry with Automatic Recall Application," J. Urology, Vol. 70, No. 3, pp. 423-6, 2007.

[McF04] E. McFadden, E. Stabile, E. Regar, "Late thrombosis in drug-eluting coronary stents after discontinuation of antiplatelet therapy," Lancet Vol. 364, pp. 1519-1521, 2004.

[McL89] G. McLean, D. Burke, "Role of Endoprostheses in the Management of Malignant Biliary Obstruction," Radiology, Vol. 170, pp. 961-7, 1989.

[Met08] Metglas, Inc. Available online at http://www.metglas.com

[Mil04] R. Milner, J. Ruurda, J. Blankensteijn, "Durability and Validity of a Remote, Miniaturized Pressure Sensor in an Animal Model of Abdominal Aortic Aneurysm," J. Endovasc. Ther., Vol. 11, pp. 372-7, 2004. 
[Mit00] T. Mita, T. Arita, N. Matsunaga, M. Furukawa, N. Zempo, K. Esato, M. Matsuzaki, "Complications of Endovascular Repair for Thoracic and Abdominal Aortic Aneurysm: An Imaging Spectrum," RadioGraphics, Vol. 20, pp. 1263-78, 2000.

[Mod81] C. Modzelewski, H. Savage, L. Kabacoff, A. Clark, "Magnetomechanical Coupling and Permeability in Transversely Annealed Metglas 2605 Alloys," IEEE Trans. on Magnetics, Vol. MAG-17, No. 6, pp. 2837-2839, 1981.

[Mol06] M. Mollazadeh, K. Murari, C. Sauer, M. Stanacevic, N. Thakor, G. Cauwenberghs, "Wireless Integrated Voltametric and Amperometric Biosensing," IEEE/NLM Life Science Systems and Applications Workshop, pp. 1-2, July 2006.

[Ne101] D. Nelson, "Expandable Metal Stents: Physical Properties and Tissue Responses," Techniques in Gastrointestinal Endoscopy, Vol. 3, No. 2, pp. 704, 2001.

[Nom98] T. Nomura, M. Takebayashi, A. Saitoh, "Chemical Sensor Based on Surface Acoustic Wave Resonator Using Langmuir-Blodgett Film," IEEE Trans. on Ultrasonics, Ferroelectrics, and Freq. Cont., Vol. 45, No. 5, pp. 1261-5, 1998.

[O'De82] T. O'Dell, “Measurement of Magnetomechanical Coupling Factor in Amorphous Ribbons," Phys. Stat. Sol. (A), Vol. 74, pp. 565-572, 1982.

[Ohk04] T. Ohki, D. Stern, M. Allen, J. Yadav, "Wireless Pressure Sensing of Aneurysms," Endovascular Today, pp. 47-52, April 2004.

[Ong05] K. Ong, X. Yang, K. Zeng, C. Grimes, "Magnetoelastic Sensors for Biomedical Monitoring," Sensor Letters, Vol. 3, pp. 108-116, 2005.

[Par95] J. Parodi, "Endovascular Repair of Abdominal Aortic Aneurysms and Other Arterial Lesions," J. Vasc. Surg., Vol. 21, No. 4, pp. 549-57, 1995.

[Ped07] R. Pedro, K. Hendlin, C. Kriedberg, M. Monga, "Wire-Based Ureteral Stents: Impact on Tensile Strength and Compression," J. Urology, Vol. 70, No. 6, pp. 1057-9, 2007.

[Pfi06] M. Pfisterer, H. P. Brunner-La Rocca, P. Buser, P. Rickenbacher, P. Hunziker, C. Mueller, R. Jeger, F. Bader, S. Osswald, C. Kaiser, "Late Clinical Events After Clopidogrel Discontinuation May Limit the Benefit of Drug-Eluting Stents," J. Am. Coll. Card., Vol. 48, No. 12, pp. 2584-91, 2006.

[Pou06] P. Pouponneau, L. Yahia, Y. Merhi, L. Epure, S. Martel, "Biocompatibility of Candidate Materials for the Realization of Medical Microdevices," Proc. IEEE EMBS, New York City, USA, pp. 2362-2365, 2006. 
[Pra07] S. Prakash, P. Abshire, "On-Chip Capacitance Sensing for Cell Monitoring Applications,” IEEE Sensors Journal, Vol. 7, No. 3, pp. 440-7, 2007.

[Puc05] L. Puckett, J. Lewis, A. Urbas, X. Cui, D. Gao, L. Bachas, "Magnetoelastic Transducers for Monitoring Coagulation, Clot Inhibition, and Fibrinolysis," Biosensors and Bioelectronics, Vol. 20, pp. 1737-43, 2005.

[Rei96] L. Reindl, G. Scholl, T. Ostertag, C. Ruppel, W.-E. Bulst, F. Seifert, "SAW Devices as Wireless Passive Sensors," IEEE Ultrasonics Symposium, pp. 3637, 1996.

[Ric07] M. Richardson, S. Green, Y.B. Gianchandani, "Magnetoelastic Wireless Sensing of Tissue Growth for Self-Expanding Biliary Stents," Proc. of IEEE MEMS, 2007, pp. 469-472.

[Ros07] H. Rosevear, S. Kim, D. Wenzler, G. Faerber, W. Roberts, J. Wolf, "Retrograde Ureteral Stents for Extrinsic Ureteral Obstruction: Nine Years' Experience at University of Michigan," J. Urology, Vol. 70, No. 5, pp. 846-50, 2007.

[Rou05] M. Roupret, M. Daudon, V. Hupertan, B. Gattegno, P. Thibault, O. Traxer, "Can Ureteral Stent Encrustation Analysis Predict Urinary Stone Composition?,” J. Urology, Vol. 66, No. 2, pp. 246-251, 2005.

[Sas93] T. Sashida, T. Kenjo, An Introduction to Ultrasonic Motors. Oxford, U.K.: Clarendon, 1993.

[Sch90] D. Scheeres, W. O’Brien, L. Ponsky, J. Ponsky, "Endoscopic Stent Configuration and Bile Flow Rates in a Variable Diameter Bile Duct Model," Surg. Endosc., Vol. 4, pp. 91-93, 1990.

[Sei07] U. Seitz, A. Block, A. Schaefer, U. Wienhold, S. Bohnacker, K. Siebert, S. Seewald, F. Thonke, H. Wulff, A. De Weerth, N. Soehendra, "Biliary Stent Clogging Solved by Nanotechnology? In Vitro Study of Inorganic-Organic Sol-Gel Coatings for Teflon Stents," Gastroenterology, Vol. 133, pp. 65-71, 2007.

[Sen03] A. Senagore (ed.), The Gale Encyclopedia of Surgery: A Guide for Patients and Caregivers, Gale Cengage, 2003.

[Ser06] P. Serruys, M. Kutryk, A. Ong, "Coronary-Artery Stents," New England J. Med., Vol. 354, pp 483-95, 2006.

[Sev01] S. Severtson, M. Nowak, "Dynamic Mechanical Properties and Low-Velocity Wetting Behavior of Plastic Crystalline States for n-Alkane Blends," Langmuir, Vol. 17, No. 16, pp. 4990-4996, 2001. 
[Sia04] G. Sianos, S. Hofma, J. Ligthart, F. Saia, A. Hoye, P. Lemos, P. Serruys, "Stent Fracture and Restenosis in the Drug-Eluting Stent Era," Cath. and Card. Interventions, Vol. 61, pp. 111-6, 2004.

[Sie08] R. Siegel, H. Luo, "Ultrasound Thrombolysis," Ultrasonics, Vol. 48, No. 4, pp. 312-20, 2008.

[Spa82 M. Spano, K. Hathaway, H. Savage, "Magnetostriction and Magnetic Anisotropy of Field Annealed Metglas 2605 Alloys via DC M-H Loop Measurements Under Stress," J. Appl. Phys., Vol. 53, No. 3, pp. 2667-9, 1982.

[Sno68] J.C. Snowdon, Vibration and Shock in Damped Mechanical Systems, Wiley \& Sons, 1968

[Som06] L. Somogyi, R. Chuttani, J. Croffie, J. DiSario, J. Liu, D. Mishkin, R. Shah, W. Tierney, L. Wong Kee Song, B. Petersen, "Biliary and Pancreatic Stents," Gastrointestinal Endoscopy, Vol. 63, No. 7, pp. 910-19, 2006.

[Sta96] N. Stark, "Literature Review: Biological Safety of Parylene C," Medical Plastics and Biomaterials, Vol. 3, No. 2, pp. 30-35, 1996.

[Ste07] C. Steeves, Y. Young, Z. Liu, A. Bapat, K. Bhalerao, A. Soboyejo, W. Soboyejo, "Membrane Thickness Design of Implantable Bio-MEMS Sensors for the In-Situ Monitoring of Blood Flow," J. Mater. Sci.: Mater. Med., Vol. 18, pp. 25-37, 2007.

[Sto00] P. Stoyanov, C. Grimes, "A Remote Query Magnetostrictive Viscosity Sensor," Sensors and Actuators A, Vol. 80, pp. 8-14, 2000.

[Sun95] J. Sung, "Bacterial Biofilm and Clogging of Biliary Stents," J. Industrial Microbiology, Vol. 15, pp. 152-5, 1995.

[Tac01] K. Tachibana, S. Tachibana, "The Use of Ultrasound for Drug Delivery," Echocardiography, Vol. 18, No. 4, pp. 323-328, 2001.

[Tak02] K. Takahata, Y. Gianchandani, "Batch Mode Micro-Electro-Discharge Machining," J. Microelectromech. Sys., Vol. 11, No. 2, pp. 102-10, 2002.

[Tak04] K. Takahata, Y. Gianchandani, "A Planar Approach for Manufacturing Cardiac Stents: Design, Fabrication, and Mechanical Evaluation," $J$. Microelectromech. Sys., Vol. 13, No. 6, pp. 933-9, 2004.

[Tak06] K. Takahata, Y. Gianchandani, K. Wise, "Micromachined Antenna Stents and Cuffs for Monitoring Intraluminal Pressure and Flow," J. Microelectromech. Sys., Vol. 15, No.5, pp. 1289-98, 2006. 
[Tol00] D. Tolley, "Ureteric Stents, Far From Ideal," The Lancet, Vol. 356, pp. 872-3, 2000 .

[Tri00] H. Trillaud, J.L. Pariente, A. Rabie, N. Grenier, "Detection of Encrusted Indwelling Ureteral Stents Using a Twinkling Artifact Revealed on Color Doppler Sonography,” Am. J. Radiology, Vol. 176, pp. 1446-8, 2000.

[Tun96] M. Tunney, P. Keane, D. Jones, S. Gorman, "Comparative Assessment of Ureteral Stent Biomaterial Encrustation," Biomaterials, Vol. 17, No. 15, pp. 1541-6, 1996.

[Uch02] M. Uchida, T. Abe, K. Nishimura, M. Hashimoto, T. Tamae, N. Hayabuchi, "Rotational Three-dimensional Cholangiography - Initial Clinical Experience," Radiation Medicine, Vol. 20, No. 4, pp. 213-5, 2002.

[Van83] E. vanSonnenberg, J. Ferrucci, C. Neff, P. Mueller, J. Simeone, J. Wittenberg, "Biliary Pressure: Manometric and Perfusion Studies at Percutaneous Transhepatic Cholangiography and Percutaneous Biliary Drainage," Radiology, Vol. 148, No. 1, pp. 41-50, 1983.

[Vir04] R. Virmani, A. Farb, G. Guagliumi, F. Kolodgie, "Drug-Eluting Stents: Caution and Concerns for Long-Term Outcome," Coronary Artery Disease, Vol. 15, pp. 313-8, 2004.

[Weg00] J. Wegener, C. Keese, I. Giaever, "Electric Cell-Substrate Impedance Sensing (ECIS) as a Noninvasive Means to Monitor the Kinetics of Cell Spreading to Artificial Surfaces," Experimental Cell Research, Vol. 259, pp. 158-66, 2000.

[Whe07] J. Whetstone, M. Smaldone, E. Gibbons, S. Jackman, "Complete Ureteral Stent Encrustation Managed with Serial Nephroscopy and Laser Lithotripsy," J. Urology, Vol. 69, No. 3, pp. 576.e15-576.e16, 2007.

[Wi195] M. Wilson, H. Kpendema, J. Noar, N. Hunt, N. Mordan, "Corrosion of intraoral magnets in the presence and absence of biofilms of Streptococcus sanguis," Biomaterials, Vol. 16, No. 9, pp. 721-725, 1995.

[Wu03] F. Wu, Z. Wang, Y. Cao, W. Chen, J. Bai, J. Zou, H. Zhu, "A randomised clinical trial of high-intensity focused ultrasound ablation for the treatment of patients with localised breast cancer," British Journal of Cancer, Vol. 89, pp. 2227-2233, 2003.

[Zen07] K. Zeng, C. Grimes, "Wireless Magnetoelastic Physical, Chemical, and Biological Sensors," IEEE Trans. on Magnetics, Vol. 43, No. 6, pp. 2358-63, 2007. 
[Zha02] H. Zhang, T. Tsang, C. Jack, J. Pollack, "Role of Bile Mucin in Bacterial Adherence to Biliary Stents," J. Lab. Clin. Med., Vol. 139, No. 1, pp. 28-34, 2002 .

[Zha04] H. Zhang, M. Marma, E. Kim, C. McKenna, M. Thompson, "Implantable Resonant Mass Sensor for Liquid Biochemical Sensing," Proc. IEEE MEMS, pp. 347-50, 2004.

[Zha06] J. Zhai, S. Dong. Z. Xing, J. Li, D. Viehland, "Giant Magnetoelectric Effect in Metglas/Polyvineliden-Fluoride Laminates," Applied Physics Letters, Vol. 89, 083507, 2006.

[Zhao06] X. Zhao, D. Lord, "Application of the Villari Effect to Electric Power Harvesting," J. Appl. Phys., Vol. 99, 08M703, 2006. 